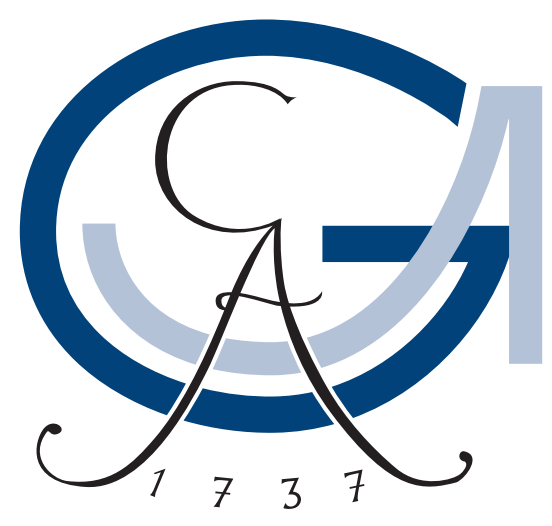

\title{
Amyloid-beta driven changes in transcriptome plasticity: From OMICs to therapy
}

\author{
Dissertation
}

for the award of the degree „Doctor rerum naturalium“

of the Georg-August Universität Göttingen within the doctoral program „Systems Neuroscience“ of the Georg-August University School of Science (GAUSS)

\author{
submitted by \\ Michael Gertig \\ from Aachen
}

Göttingen, 2016 
Thesis Committee and members of the examination board:

Referee: Prof. Dr. André Fischer Dept. for Psychiatry and Psychotherapy

$2^{\text {nd }}$ Referee: Prof. Dr. Ralf Heinrich Dept. for Cellular Neurobiology

$3^{\text {rd }}$ Referee: Prof. Dr. Silvio Rizzoli

Dept. for Neuro- and Sensory Physiology

Further members of the examination board:

Prof. Dr. Dr. Hannelore Ehrenreich

MPI for experimental medicine - Clinical Neuroscience

PhD Camin Dean

European Neuroscience Institute - Trans-synaptic Signaling

Dr. Hauke Werner

MPI for experimental medicine - Neurochemistry 


\section{Declaration of independence}

I hereby declare that the dissertation entitled

"Amyloid-beta driven changes in transcriptome plasticity: From OMICS to Therapy"

was written on my own and independently without any other aids and sources than indicated.

Michael Gertig

$\overline{\text { Göttingen, April } 2016}$ 
To my lovely wife and our wonderful daughter.

,Walk with the dreamers, the believers, the courageous, the cheerful, the planners, the doers, the successful people with their heads in the clouds and their feet on the ground. Let their spirit ignite a fire within you..."

Wilferd Arlan Peterson (1900-1995) 


\section{Table of contents}

List of figures $\quad$ VII

List of tables $\quad$ IX

1 Introduction 1

1.1 Alzheimer's disease . . . . . . . . . . . . . . . . . . . . . . . 1

1.1.1 Symptoms, diagnosis, progression and treatment . . . . . . . . . . . 3

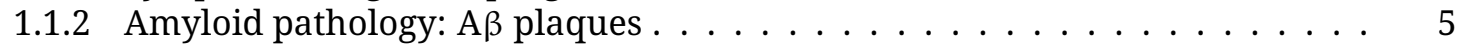

1.1.3 tau pathology: neurofibrillary tangles . . . . . . . . . . . . . . . 9

1.1.4 Risk factors for Alzheimer's disease . . . . . . . . . . . . . . . . . . . . . 12

1.1.5 The APP/PS1 mice and other mouse models for Alzheimer's disease . . . . . . 14

1.2 Learning and memory . . . . . . . . . . . . . . . . . . . . . . . . . 15

1.2.1 Categories of memory . . . . . . . . . . . . . . . . . . 15

1.2.2 Brain areas involved in different modes of memory . . . . . . . . . . . 17

1.2.3 Cellular mechanisms of learning and memory . . . . . . . . . . . . 20

1.3 Epigenetics and its impact on gene expression . . . . . . . . . . . . . . 22

1.3.1 DNA methylation . . . . . . . . . . . . . . . . . . . 23

1.3.2 Histone modifications . . . . . . . . . . . . . . . . . . . . 25

1.3.3 DNA methylation and histone modification in age and disease . . . . . . . 27

$\begin{array}{llr}2 & \text { Objectives } & 29\end{array}$

3 Materials and Methods 30

3.1 Animals . . . . . . . . . . . . . . . . . . . . . . . . 30

3.1 .1 Genotyping . . . . . . . . . . . . . . . . . . . . . . . . . . 30

3.1 .2 Brain dissection . . . . . . . . . . . . . . . . . . . . 31

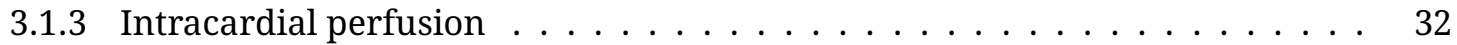

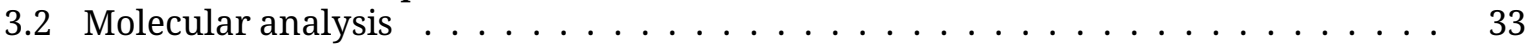

3.2 .1 Immunohistochemistry . . . . . . . . . . . . . . . . . . . . 33

3.2 .2 Confocal imaging . . . . . . . . . . . . . . . . . . . . . . . . . 34

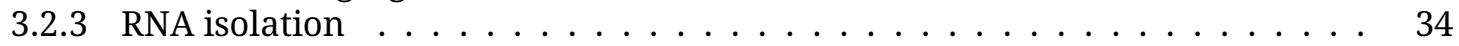

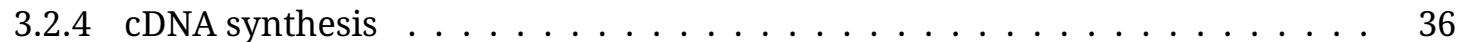

3.2 .5 Quantitative real time PCR . . . . . . . . . . . . . . . . . . . . 37

3.2 .6 RNA sequencing . . . . . . . . . . . . . . . . . . . . 37

3.2.7 Fluorescence-activated cell sorting (FACS) of nuclei . . . . . . . . . . . . . . 38

3.2 .8 Chromatin preparation from sorted nuclei . . . . . . . . . . . . . . . . . 39

3.2 .9 Chromatin immunoprecipitation (ChIP) . . . . . . . . . . . . . . . . . . 40

3.2.10 Library preparation for Multiplex Illumina ChIP-sequencing . . . . . . . . . 40

3.2.11 Methylated DNA immunoprecipitation (MeDIP) _ . . . . . . . . . . . . . . 42

3.3 NGS data analysis . . . . . . . . . . . . . . . . . . . . . . . 44

3.3.1 Differential gene expression $\ldots \ldots \ldots \ldots \ldots \ldots$. . . . . . . . . . . 44

3.3.2 Transcription factor binding site prediction $\ldots \ldots \ldots \ldots$. . . . . . . . 45

3.3.3 Differential splicing . . . . . . . . . . . . . . . . . . . . . . . . . . . 45

3.3.4 MeDIP- and ChIPseq data analysis . . . . . . . . . . . . . . . . 46

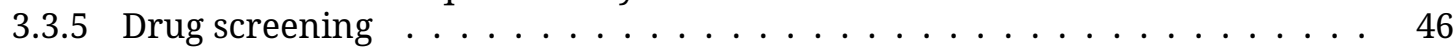




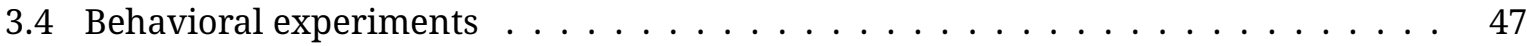

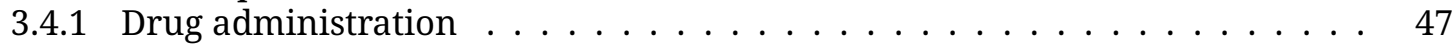

3.4 .2 Open field . . . . . . . . . . . . . . . . . . . . . . 47

3.4 .3 Elevated plus maze . . . . . . . . . . . . . . . . . . . . . . . . . . . . . . . . . . . . . . . .

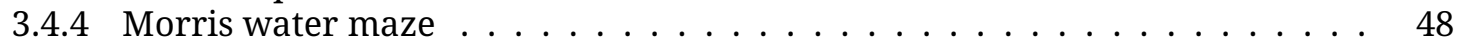

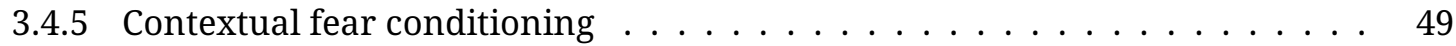

3.5 Statistics and graphical output . . . . . . . . . . . . . . . . . . . 50

3.6 R source codes . . . . . . . . . . . . . . . . . . . . . . . 51

3.7 Buffers and solutions . . . . . . . . . . . . . . . . . . 55

4 Results $\mathbf{5 6}$

4.1 Transcriptional differences among brain regions . . . . . . . . . . . . . . . . . 56

4.2 Wildtype-like gene expression in young APP/PS1 mice . . . . . . . . . . . . . . . 59

4.3 Differential gene expression in APP/PS1 mice with amyloidosis . . . . . . . . . . . . 62

4.4 Differential gene expression during aging . . . . . . . . . . . . . . . . . . . . . . . . . . . . . . . . .

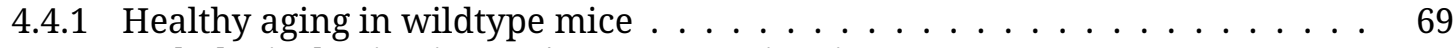

4.4.2 Pathological aging in APP/PS1 transgenic mice . . . . . . . . . . . . . . . 75

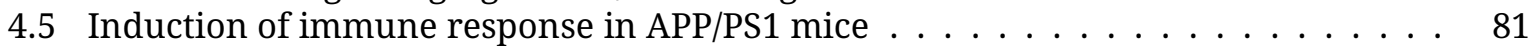

4.6 Transcription factor analysis in APPPS1 mice . . . . . . . . . . . . . . . . . . . . . . . . . . . . . .

4.7 Differential splicing in APPPS1 transgenic mice . . . . . . . . . . . . . . . . . . . . 86

4.8 Differential splicing during aging . . . . . . . . . . . . . . . . . . . . . . . . . . . . . . . . . . . . . . .

4.8.1 Differential splicing in healthy aging . . . . . . . . . . . . . . . . 89

4.8.2 Differential splicing in aging transgenic mice . . . . . . . . . . . . . . . . . . 94

4.9 DNA methylation in APP/PS1 mice . . . . . . . . . . . . . . . . . . . . . . . . . . . . . . . . . . . . . . . . . . . . . . . . . . .

4.10 H3K4me3 in APP/PS1 mice . . . . . . . . . . . . . . . . . . . . . . 106

4.11 Drug screening for novel therapeutic strategies in AD . . . . . . . . . . . . . . . . . . . . . . . . . . . . . . .

4.12 Combinatory treatment with SAHA and memantine . . . . . . . . . . . . . 110

5 Discussion $\quad 115$

5.1 Interregional differences outweigh age and disease . . . . . . . . . . . . . . . 115

5.2 Compensatory effects in young APP/PS1 mice . . . . . . . . . . . . . . . . . . . . . . . . . . . . . . . . . . . . .

5.3 Cognitive decline and immune response in APP/PS1 mice . . . . . . . . . . . . . 117

5.4 Alternative splicing might facilitate amyloid deposition . . . . . . . . . . . . . . . 119

5.5 DNA methylation acts via long-distance interactions in APP/PS1 mice . . . . . . . 121

5.6 The impact of amyloidosis on H3K4me3 remains elusive . . . . . . . . . . . . . . . 123

5.7 Drug screening and treatment of APP/PS1 mice . . . . . . . . . . . . . . . . . 124

$\begin{array}{llr}6 & \text { Conclusions } & 127\end{array}$

$\begin{array}{llr}7 & \text { Supplemental figures and tables } & 129\end{array}$

8 List of abbreviations $\quad 137$

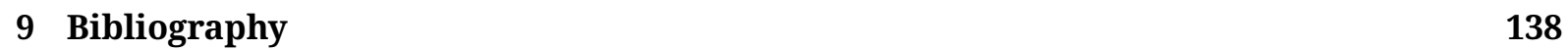

10 Acknowledgments $\quad 172$

11 Curriculum vitae $\quad 174$ 


\section{List of figures}

1.1 Life expectancy in 60 and 80 years old OECD citicens . . . . . . . . . . . . . . 1

1.2 Age dependent incidence and predicted increase of AD cases . . . . . . . . . . 2

1.3 Estimated 3-dimensional structure of APP . . . . . . . . . . . . . . . . 6

1.4 Amyloidogenic cleavage of APP and formation of plaques . . . . . . . . . . . 7

1.5 Braak and Braak stages for amyloid deposition . . . . . . . . . . . . . . . . 8

1.6 Hyperphosphorylated tau aggregates and forms neurofibrillary tangles . . . . . . . 11

1.7 Braak and Braak stages for neurofibrillary tangles . . . . . . . . . . . . . . . . 12

1.8 Location of the ACC and hippocampus in human and mouse brains . . . . . . . . 18

1.9 Scheme of the hippocampal formation . . . . . . . . . . . . . . . . . . . . . 19

1.10 Waddington's"epigenetic landscape" . . . . . . . . . . . . . . . . . . . 22

1.11 De novo and maintenance methylation of cytosine by DNTMs . . . . . . . . . . . . 24

1.12 Major modifications of histones H1, H2A, H2B, H3, and H4 . . . . . . . . . . . 26

4.1 Transcriptional differences among brain regions . . . . . . . . . . . . . . 57

4.2 Interregional differences: Percentage of upregulated genes $\ldots \ldots \ldots \ldots \ldots$

4.3 Clustering of 1.5 months old mice . . . . . . . . . . . . . . . . . 60

4.4 Transcriptional differences in 1.5 months old mice . . . . . . . . . . . . . . 61

4.5 Differential gene expression in APP/PS1 transgenic mice . . . . . . . . . . . . . . 63

4.6 Differentially expressed genes in APP/PS1 mice shared among brain regions . . . . 64

4.7 Overrepresented functional pathways in APP/PS1 transgenic mice . . . . . . . . . . 65

4.8 Functional pathways from mildly downregulated genes . . . . . . . . . . . . . 66

4.9 Validation of RNA sequencing via qPCR . . . . . . . . . . . . . . . . . 68

4.10 Differential gene expression in aging wildtype mice $\ldots \ldots \ldots$. . . . . . . 70

4.11 Common up- and downregulated genes during aging in wildtype mice $\ldots . . . .71$

4.12 Overrepresented functional pathways in aging wildtype mice . . . . . . . . . . 72

4.13 Functional pathways from mildly deregulated genes in aging wildtype mice . . . . 73

4.14 Common functional pathways from mildly deregulated genes in aging wildtype mice 74

4.15 Differential gene expression in aging APP/PS1 mice . . . . . . . . . . . . . . 75

4.16 Common up- and downregulated genes during aging in transgenic mice . . . . . 76

4.17 Overrepresented functional pathways in aging transgenic mice . . . . . . . . . . . 78

4.18 Functional pathways from mildly deregulated genes in aging transgenic mice . . . 79

4.19 Functional pathways from mildly downregulated genes in transgenic mice . . . . . 79

4.20 Overlaps in gene expression among wildtype and transgenic aging mice . . . . . . 80

4.21 Neural cell counts from transgenic and wildtype mice . . . . . . . . . . . . . . 82

4.22 Confocal imaging of the dentate gyrus from 4 months old mice . . . . . . . . . . . 82

4.23 Overrepresented transcription factors in APP/PS1 transgenic mice . . . . . . . . . . 84

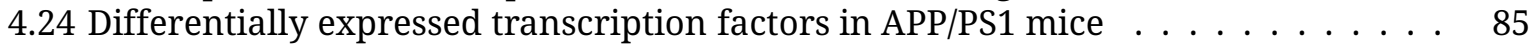

4.25 Differiental splicing in APP/PS1 transgenic mice . . . . . . . . . . . . . . . . . 87

4.26 Differiental splicing in aging wildtype mice . . . . . . . . . . . . . . . . 90

4.27 Comparison of differential gene expression and splicing analysis . . . . . . . . . . . 91

4.28 Differiental splicing in aging wildtype mice . . . . . . . . . . . . . . . . . . 95

4.29 NGS plots for DNA methylation along gene bodies . . . . . . . . . . . . . . . . . 99

4.30 DNA methylation in APP/PS1 transgenic mice . . . . . . . . . . . . . . . . 100

4.31 Differential gene expression and DNA methylation in APP/PS1 mice . . . . . . . . 102

4.32 Differential distribution of DNA methylation in APP/PS1 mice . . . . . . . . . . . . . 103

4.33 Long distance interactions between genes and sites of DNA methylation . . . . . . 104

4.34 Gene expression hot-spots and sites of DNA methylation . . . . . . . . . . . . . 105

4.35 Genome wide distribution of H3K4me3 . . . . . . . . . . . . . . . . . 106 
4.36 H3K4me3 and differential gene expression in APP/PS1 mice . . . . . . . . . . . . . 107

4.37 Explorative behavior and basal anxiety levels in treated APP/PS1 transgenic mice . 110

4.38 Evaluation of anxiety-like behavior in treated APP/PS1 transgenic mice . . . . . . . 111

4.39 Spatial memory in treated APP/PS1 mice tested . . . . . . . . . . . . . . . . . . . 112

4.40 Contextual fear conditioning in APP/PS1 mice after drug treatment . . . . . . . . 113

7.1 Detailed dendrogram for eucledian distances of RNA samples . . . . . . . . . . . . 129 


\section{List of tables}

3.1 DreamTaq genotyping protocol . . . . . . . . . . . . . . . . . . . 31

3.2 Primary and secondary antibodies for immunohistochemistry . . . . . . . . . 33

3.3 DNAse I reaction solution . . . . . . . . . . . . . . . . . . . . . . . . . . 35

3.4 Standard protocol for Transcriptor First Strand cDNA Synthesis . . . . . . . . . . . 36

3.5 Protocol for qPCR of mRNA samples with LightCycler $480 \ldots \ldots \ldots \ldots \ldots$

3.6 Protocol for qPCR of ChIP samples using NEBNext Q5 polymerase . . . . . . . . . . 42

3.7 Protocol for qPCR of MeDIP samples using Phusion HF polymerase . . . . . . . . . . 43

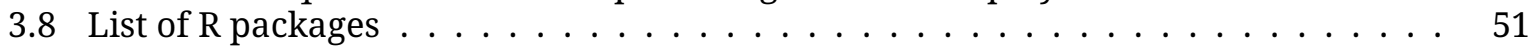

4.1 Functional pathways in 1.5 months old mice . . . . . . . . . . . . . . . . . . 61

4.2 Biological function of deregulated Transcription factors . . . . . . . . . . . . 86

4.3 Overrepresented KEGG-pathways for differential splicing in young APP/PS1 mice . 88

4.4 Overrepresented KEGG pathways for differential splicing in aging wildtype mice . 92

4.5 KEGG pathways for differential splicing in wildtype ACC: 4 vs 8 months (1) . . . . 93

4.6 KEGG pathways for differential splicing in wildtype ACC: 4 vs 8 months (2) . . . . 94

4.7 KEGG pathways for differential splicing in early-life aging transgenic mice: DG . . 96

4.8 KEGG pathways for differential splicing in mid-life aging transgenic mice: ACC (1) 97

4.9 KEGG pathways for differential splicing in mid-life aging transgenic mice: ACC (2) 98

4.10 Top 10 drugs identified by screening with DrugPairSeeker after filtering . . . . . . 109

7.1 KEGG pathways enriched by interregional differences . . . . . . . . . . . . . . . 130

7.2 Canonical pathways for upregulated genes in 1.5 months old APP/PS1 mice: ACC . 131

7.3 Canonical pathways for upregulated genes in 4 months old APP/PS1 mice: ACC . . 131

7.4 Canonical pathways for upregulated genes in 8 months old APP/PS1 mice: ACC . . 131

7.5 Canonical pathways for downregulated genes in 1.5 months old APP/PS1 mice: ACC 132

7.6 Canonical pathways for downregulated genes in 4 months old APP/PS1 mice: ACC . 132

7.7 Canonical pathways for downregulated genes in 8 months old APP/PS1 mice: ACC . 132

7.8 Canonical pathways for upregulated genes in 1.5 months old APP/PS1 mice: CA1 . 133

7.9 Canonical pathways for upregulated genes in 4 months old APP/PS1 mice: CA1 . . 133

7.10 Canonical pathways for upregulated genes in 8 months old APP/PS1 mice: CA1 . . 133

7.11 Canonical pathways for downregulated genes in 8 months old APP/PS1 mice: CA1 . 134

7.12 Canonical pathways for upregulated genes in 1.5 months old APP/PS1 mice: DG . . 134

7.13 Canonical pathways for upregulated genes in 4 months old APP/PS1 mice: DG . . . 134

7.14 Canonical pathways for upregulated genes in 4 months old APP/PS1 mice: DG . . . 135

7.15 Canonical pathways for downregulated genes in 1.5 months old APP/PS1 mice: DG 135

7.16 Canonical pathways for upregulated genes in 8 months old APP/PS1 mice: DG . . . 135

7.17 Core-set of transcription factors . . . . . . . . . . . . . . . . . . 136

7.18 Hot-spots of gene expression in APP/PS1 mice . . . . . . . . . . . . . . 136 


\section{Introduction}

\subsection{Alzheimer's disease}

During the recent decades, life expectancy of elderly human increased drastically (OECD, 2009). The average life expectancy of 60 years old OECD citizens in 1971 was estimated to 14 years (15.6 in women, 12.7 in men). Less than 40 years later, people of the same age can expect to live for 5 more years in average. The progression of life expectancy during the recent decades follows an almost linear trend with no plateau in sight yet (see figure 1.1). While a longer life is in principle an eligible privilege for most people, an increasing life expectancy does bear some negative aspects. With increasingly aging societies, the prevalence of age related diseases is unfortunately rising as well. Alzheimer's disease (AD) is only one example for these age-related diseases in human. According to the World Alzheimer's Report 2015 from the ADI

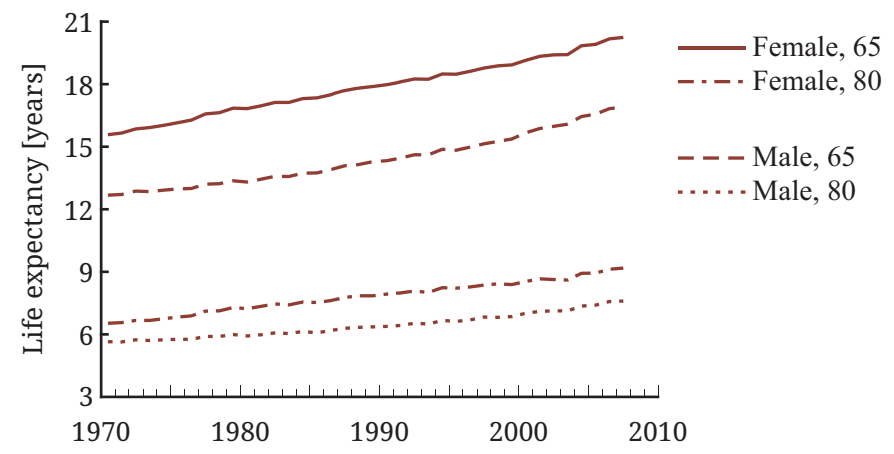

Figure 1.1 - Life expectancy in 65 and 80 years old OECD citizens from 1971 to 2007:

The graphic was adapted from the OECD report "Health at a glance" (OECD, 2009)

(Alzheimer's Disease International), the average incidence for $\mathrm{AD}$ at an age of $60-64$ years is $0.39 \%$, however, this number drastically increases with increasing age (see figure 1.2A, p.2) (Prince et al., 2015). Approximately 1\% of all humans between an age of 70 and 74 are suffering from $\mathrm{AD}$ and in countries with a high income, this number rises to $12.5 \%$ for people older than 90 . Concordantly, more than 46 million people worldwide are suffering from the disease and the ADI estimated that the incidence will triplicate within the next 35 years (see figure 1.2B, p.2). This drastic increase of AD cases is a rising burden for our societies, both socially and economically. During the past five years, the worldwide costs of $\mathrm{AD}$ increased by more than 35\% from 604 to 818 billion US\$ and were even estimated to triplicate within the next fifteen years, highlighting the need for further knowledge on how to counteract or prevent the disease. 
A

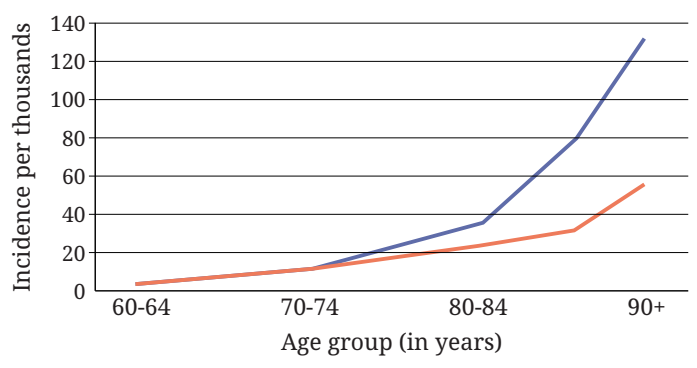

High income countries
B

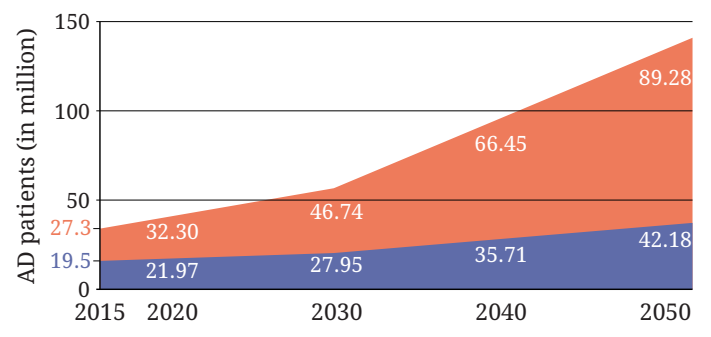

Low \& middle income countries

Figure 1.2 - Age dependent incidence and predicted increase of AD cases:

A) Number of AD patients of a certain age ranging from 60 to 90 and above, grouped for different world regions and their economic wealth.

B) Estimated progression of incidence during the next 35 years in countries with high income and those with middle and low income. Graphics were adapted from the ADI's World Alzheimer's Report (Prince et al., 2015).

Due to the correlation of $\mathrm{AD}$ incidence and life expectancy, the awareness of $\mathrm{AD}$ is constantly rising since its discovery in the early $20^{\text {th }}$ century. In 1901, the psychiatrist and neuropathologist Alois Alzheimer examined new patients at the Frankfurt Asylum (Germany) and was intrigued by the symptoms of the 51 years old Auguste Deter (A.D.) (Maurer and Maurer, 1998). According to her husband, who brought her to the asylum, the behavior and habits of A.D. were changing severely. In his publication in 1907, Alois Alzheimer described that the patient A.D. had suddenly become jealous of her husband, was disoriented, delirious, experienced hallucinations and developed a rapid loss of memory (Alzheimer et al., 1995). She often seemed unable to perceive the actual situation and when she was interviewed during her examinations, she could not understand the questions, responded incoherently or seemed devoid of thoughts. Additionally, she had troubles in reading, writing and speaking. Alzheimer found the majority of symptoms to oscillate in strength with one exception that was progressively aggravating until her death: "imbecility”. Even though he himself interpreted these symptoms not to be severe, the entire loss of cognitive abilities described developed in a rather short period of time.

A.D. died in 1906 from a sepsis caused by bedsores, only 41/2 years after entering the asylum. When dissecting the brain of A.D., Alzheimer found the two major characteristics for that particular disease later on named Alzheimer's disease: A massive degeneration of neurons driven or at least coinciding with fibrillary tangles that led to the loss of $25 \%$ to $30 \%$ of all cortical neurons; and additionally, deposits of an unknown substance throughout the cortex that were 
visible without dyeing (Alzheimer et al., 1995). It took 70 to 80 years until tau and APP, the proteins involved in these pathologies and their corresponding genes were described in more detail (Masters et al., 1985; Weingarten et al., 1975). Based on his findings, Alois Alzheimer concluded, that the case of A.D. describes an - until then - special illness and he found an increasing number of patients with similar symptoms throughout the subsequent years. Furthermore, he suggested to reevaluate the classical clinical distinction of known pathologies to enable a more detailed definition of illnesses in general. Notably, researches today mostly agree that AD itself is a heterogeneous disease with distinct subtypes regarding symptoms, disease progression and origin (Bredesen, 2015; Lam et al., 2013).

\subsubsection{Symptoms, diagnosis, progression and treatment of Alzheimer's dis- ease}

The differentiation of $\mathrm{AD}$ from other mental diseases is, due to its heterogeneity and the lack of binary biomarkers, not trivial. In 1985, a joint work group from the National Institute of Neurological and Communicative Disorders and Stroke (NINCD) and the Alzheimer's Disease and Related Disorders Association (ADRDA) tried to redefine the clinical diagnosis of AD, since approximately $20 \%$ of all diagnosed AD cases were found to be false-positive after autopsy (McKhann et al., 1984). Together with diagnostics for AD described in the text revision of the fourth edition of the "Diagnostic and Statistical Manual for Mental Disorders" (DSM-IV-TR), published by the American Psychiatric Association, the NINCDS-ADRDA criteria were the standard for diagnosing AD for the recent decades (Dubois et al., 2007). Even though these diagnostic criteria were revised multiple times, a recent study on the accuracy of diagnosing AD estimated a sensitivity of 70 to $87 \%$ and a specificity of $44 \%$ to $71 \%$ (Beach et al., 2012). Thus, almost 30 years after the first definition of the NINCDS-ADRDA criteria, approximately one fourth of all AD cases are still not identified as such and a third to half of all diagnosed patients do actually not suffer from $\mathrm{AD}$ according to the gold standard: amyloid plaques and neurofibrillary tangles (NFT).

In 2007, Dubois et al. proposed a new diagnostic approach based on the classical criteria and novel, recently developed techniques. This approach combined core diagnostic criteria with 
supportive features and exclusion criteria to determine the probability for $\mathrm{AD}$ in a human patient. These core criteria describe a gradual progressive decline of memory function reported by patients or informants and a loss of episodic memory evaluated by psychological tests. AD is considered as a probable diagnosis when these core criteria coincide with either one of the following supportive feature: medial temporal lobe atrophy, thus a loss of volume in the hippocampus, entorhinal cortex and amygdala; abnormal cerebrospinal fluid biomarkers like low concentrations of neurotoxic $A \beta_{1-42}$; a high concentration of tau and/or increased concentrations of phosphorylated tau along with other biomarkers that might be developed in the future. Non-coding RNAs are only one example of molecules considered as promising biomarkers (Rao et al., 2013). Other proposed supportive features are a reduced glucose metabolism in temporal parietal regions and other well validated ligands detected by positron emission tomography and autosomal dominant mutations in known genetic risk factors for $\mathrm{AD}$ (see section 1.1.4, p.12) (Dubois et al., 2007).

In addition to these features, Dubois et al. proposed certain exclusion criteria that would contradict the diagnosis of $\mathrm{AD}$, including a sudden disease onset, early gait abnormalities and seizures, sensory loss, major depression or cerebrovascular disease. Other psychological symptoms that were not included in Dubois' diagnostic strategy, though can frequently be found in AD patients are hallucinations (Holroyd et al., 2000), delusions (Forstl et al., 1994), apathy (Tunnard et al., 2011), anxiety (Teri et al., 1999), sleep disturbances (Cole and Richards, 2005), agitation (Bruen et al., 2008), and aggression (Poulin et al., 2011). While the intensity of some psychological symptoms might slightly decrease during disease progression, the decline of memory function and neuroanatomical symptoms, including amyloid plaques and NFT, deteriorate gradually (Alzheimer et al., 1995; Holtzer et al., 2003). The progression of these anatomical symptoms was described in detail and categorized by Braak and Braak in 1991. These "Braak and Braak stages” are commonly used for the post-mortem evaluation of disease stage and will subsequently be described in more detail.

Unfortunately, even though a number of drugs against AD were developed during the recent decades, no cure is available yet and the efficacy of treatments are debated (Mangialasche et al., 2010). Four FDA-approved therapeutic drugs are currently in use for treating AD patients (on Aging, 2015). Donepezil, rivastigmine, and galantamine are all acting as cholinesterase inhibitors 
and are used for treating mild to severe AD cases (Burns et al., 1999; Rösler et al., 1998; Wilcock et al., 2000). Acetylcholine was shown to be severely decreased in AD patients but also during healthy aging (Bartus et al., 1982). The importance of acetylcholine for neuronal functioning was already discovered in the early $20^{\text {th }}$ century by Henry Hallett Dale and Otto Loewi who were later awarded the Nobel Prize for Medicine ("for their discoveries relating to chemical transmission of nerve impulses”). Nowadays, the function of acetylcholine as a neurotransmitter and its importance in cognitive processes is well accepted since disruptions in neuronal acetylcholine levels can cause cognitive impairments (Sarter and Bruno, 1997). The only non-cholinesterase inhibitor among the approved AD-drugs is memantine.

Memantine was shown to improve memory function and induce anti-depressant like effects by antagonizing NMDA-receptors and thus re-establishing a homeostatic level of glutamatergic signaling (Barnes et al., 1996; Kos and Popik, 2005; Parsons et al., 2007). However, targeting NMDAreceptors is not a trivial approach since both hyper- and hypoactivity of glutamatergic signaling were shown to cause neuronal dysfunction (Parsons et al., 2007). Concordantly, even though memantine is considered a well tolerated drug, a variety of milder side effects is relatively common ( $\geq 2 \%$ of all recipients) including headaches, back pain, vomiting, incontinence, bronchitis, anxiety, and anorexia (Thomas and Grossberg, 2009). Interestingly, depression is a common side effect of memantine as well, highlighting the difficulty of reinstating a healthy glutamatergic signaling. In addition to the adverse side effects, all available medications for AD are limited in their efficacy indicating the need for novel therapeutic approaches (Godyn et al., 2016).

\subsubsection{Amyloid pathology: $A \beta$ plaques}

When dissecting the brain of A.D., Alois Alzheimer discovered plaques of an unknown substance throughout the cortex. Eighty years after his discovery, Masters et al. (1985) purified and described the "amyloid plaque core protein" that is nowadays known as A $\beta$ (amyloid $\beta$ ) for the first time. This plaque forming $A \beta$ is the cleavage product of the amyloid precursor protein (APP), whose gene is localized on human chromosome 21 and was identified by 4 groups individually in 1987 (Goldgaber et al., 1987; Kang et al., 1987; Masters et al., 1985; Robakis et al., 1987; Tanzi et al., 1987). APP is intensively studied ever since its discovery, however, little is known about its role in a healthy, physiological context even though APP can be implicated in a variety 
of cellular processes. Studies on APP deficient mice suggest that it might function in glutamatergic synapse formation and transmission and thus in the acquisition of aversive memory (Priller et al., 2006; Senechal et al., 2008). Studies on neuronal cell cultures further indicate a role of APP in proliferation, differentiation, and survival of neurons (Araki et al., 1991; Hayashi et al., 1994; Milward et al., 1992; Perez et al., 1997).

Many studies regarding APP focus on its enzymatic cleavage and the neurotoxic effect of resulting amyloid fragments, in particular $A \beta_{1-42}$. APP is a transmembrane protein and consists of three extracellular domains (E1, KPI, and E2), a transmembrane domain and the intracellular C-terminal domain of relatively unknown functional significance (see figure 1.3) (Dawkins and Small, 2014). The transmembrane domain, however, contains cleavage sites for a number of secretases which are the key factors for the synthesis of $A \beta$ fragments. Initially, APP can be cleaved by $\alpha$ - and $\beta$-secretases, producing soluble APP $\alpha$ and $-\beta$ peptides (Haass et al., 2012). Cleavage by $\alpha$-secretases is considered as the anti-amyloidogenic pathway as it prevents the formation of neurotoxic $A \beta$ species (Haass et al., 2012). In contrast, cleavage by the $\beta$-secretase BACE1 ( $\beta$-site APP cleaving enzyme 1) produces larger residual fragments that can further be cleaved by the $\gamma$-secretase generating $\mathrm{A} \beta$ and an APP intracellular domain (see figure 1.4, p.7). The $\gamma$-secretase is a protein complex that consists of four integral membrane proteins with presenilin (PS/PSEN) as the catalytic component (De Strooper et al., 2012). The amyloid peptide isoform $A \beta_{1-42}$ resulting from enzymatic cleavage though $\beta$ - and $\gamma$-secretases was initially considered the neurotoxic species as it is likely to form oligomers which can further aggregate to amyloid plaques. Interestingly, a second isomer, $A \beta_{1-40}$, was shown to inhibit this oligomerization and might thereby ameliorate the amyloid plaque burden (Murray et al., 2009). In AD patients, a significant increase of $A \beta_{1-42}$ in relation to $A \beta_{1-40}$ can be detected. $A \beta_{1-40}$ and $A \beta_{1-42}$ are by far the most studied amyloid peptides, though

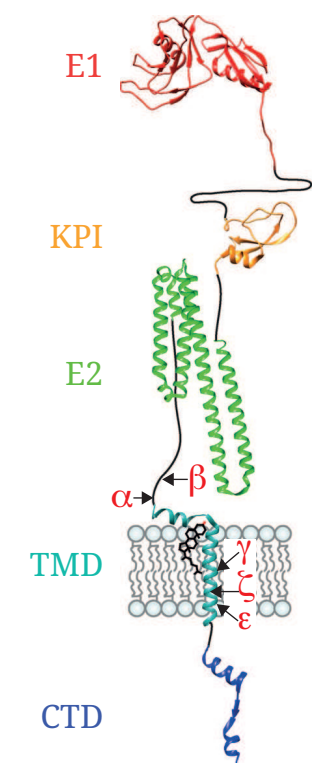

Figure 1.3 - Estimated 3dimensional structure of APP: Extracellular domains E1, KPI, and E2, transmembrane domain (TMD) and C-terminal domain (CTD) of APP. Arrows point at the $\alpha$ - to $\epsilon$-cleavage sites. Graphic was adapted from Dawkins and Small (2014). 
a variety of $\mathrm{A} \beta$ isoforms were recently isolated from human tissue and implicated with $\mathrm{AD}$ as well (Portelius et al., 2010).

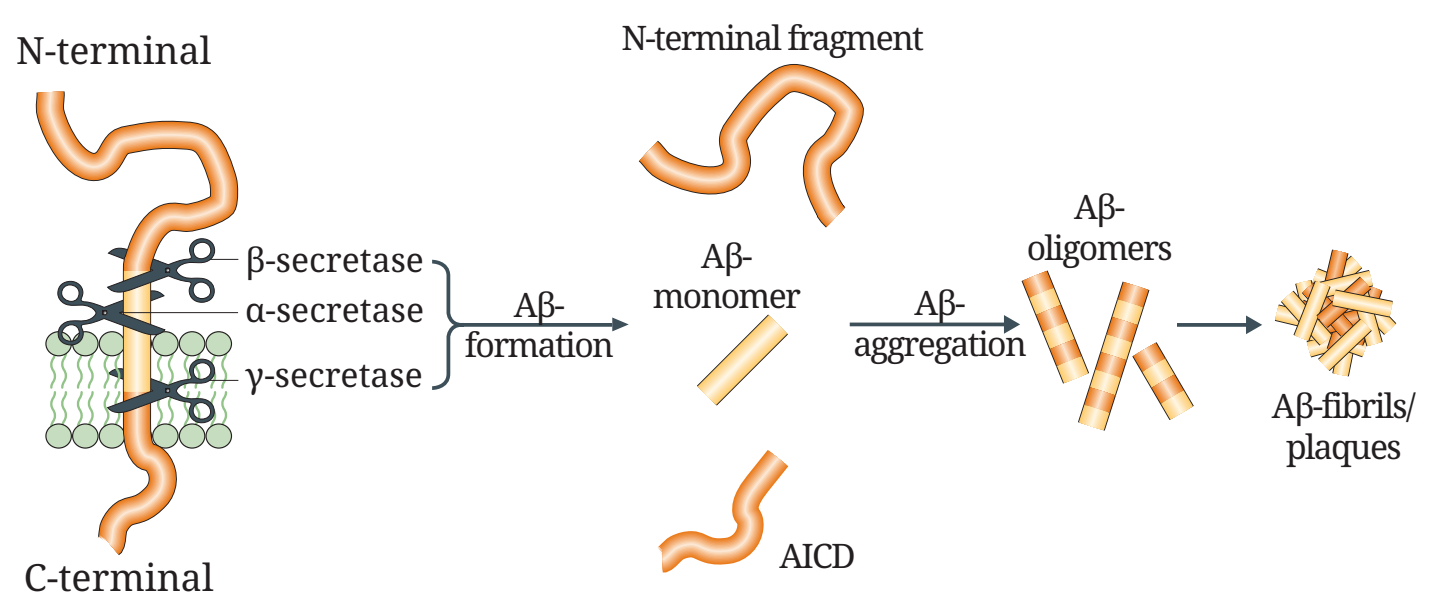

Figure 1.4 - Amyloidogenic cleavage of APP and formation of plaques:

Cleavage of APP by $\beta$ - and $\gamma$-secretases lead to the production of monomeric A $\beta$ which can aggregate to oligomers, subsequently forming plaques. The graphic was adapted from Götz and Ittner (2008).

Based on the gradual increase of amyloid plaques in size and number, Braak and Braak (1991) defined three stages of amyloid pathology in AD patients (see figure 1.5, p.8). In "stage A", the first amyloid deposits with low density can be found in the ventral parts of the frontal, temporal, and occipital lobes. These deposits become denser during disease progression and spread across the entire isocortex except from primary sensory areas and the motor cortex in "stage B". Most plaques can be found in cortical layers Va and VI and in the white matter underlying these layers. The hippocampus is only mildly affected at this stage with the highest number of deposits found in the CA1. In the end-stage of AD (amyloid "stage C"), amyloid plaques are spread across the entire brain with a slightly milder impact on the hippocampus, thalamus, and hypothalamus compared to other forebrain regions.

Though the level of amyloid deposits is drastically increased in AD patients, synthesis of neurotoxic amyloid species and subsequent oligomerization is not exclusive for $\mathrm{AD}$ but can be found in other diseases and even cognitive healthy elderly as well (Iwatsubo et al., 1995; Price and Morris, 1999). Thus, the more commonly used categorization of AD pathology follows the burden from NFT. How amyloid plaques impact physiological processes is rather unknown and some researches even debate the effect of amyloid deposits on neurodegeneration and cognitive decline 
(Wirth et al., 2013). However, cell culture experiments and studies on transgenic mice expressing mutated forms of APP strongly suggest a role of amyloid plaques in disturbing homeostasis, impairing immune functions and driving neurodegeneration, thus cognitive decline. Soluble oligomeric $A \beta$ produced by human neuronal cell cultures and injected into the lateral ventricle of rats was shown to specifically impair cognitive functions independently of neurodegeneration (Cleary et al., 2005). Bittner et al. (2012) found that amyloid plaques in mice led to a significant loss of dendritic spines in proximate neurons. On the molecular level, these neuroanatomical and psychological effects might partially be driven by disturbances of JAK/STAT signaling caused by an induction of $A \beta_{1-42}$ (Chiba et al., 2009; Hsu et al., 2013). This JAK/STAT signaling pathway functions in synaptic plasticity and might be a direct link between neuronal functions and glial immune response that is induced but impaired by the presence of amyloid deposits (Meda et al., 1995; Nicolas et al., 2012).

A

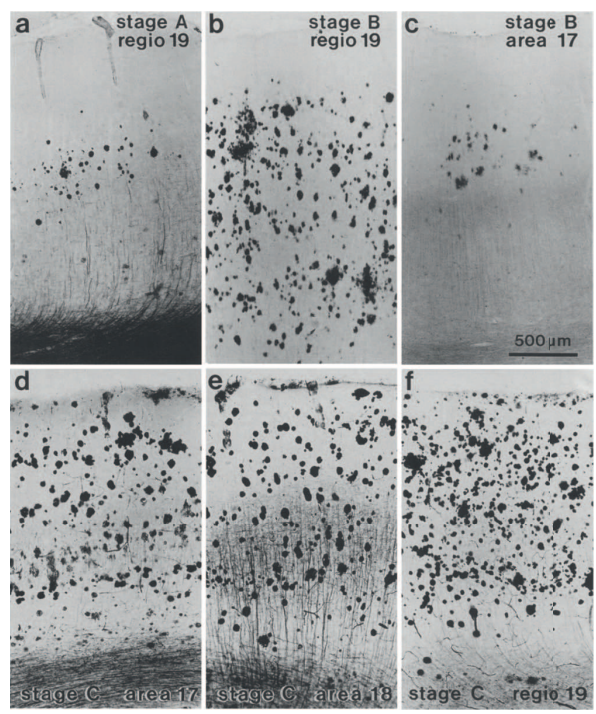

B

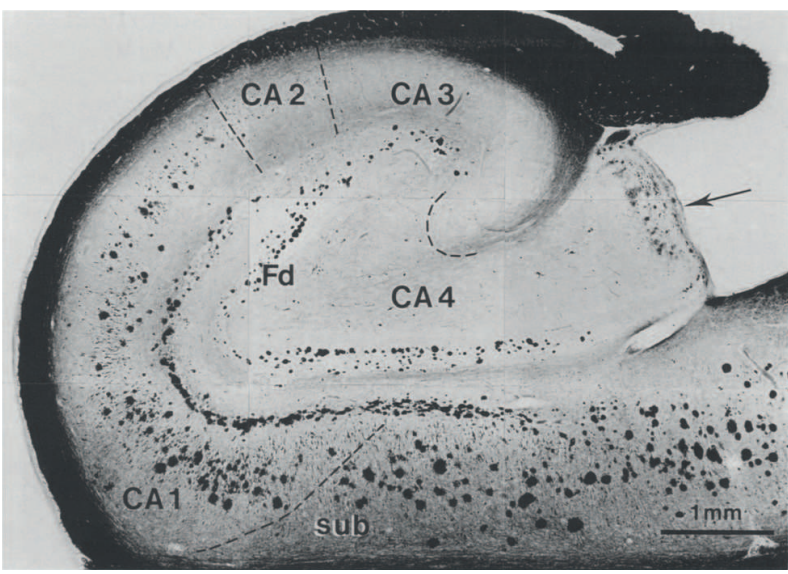

Figure 1.5 - Braak and Braak stages for amyloid deposition:

A) Amyloid deposits in "stage A" (a), "stage B" (b-c), and "stage C" (d-f) inside the occipital isocortex.

B) Hippocampal formation in "stage C". Dashed lines represent the boundaries between the subiculum and CA regions, the arrow highlights "fluffy material" at the border of the dentate gyrus. Unedited graphics from Braak and Braak (1991).

Phagocytosis and thus clearance of disturbing protein aggregates within the CNS (central nervous system) is mainly achieved by microglia. Amyloid peptides might be recognized by the TREM2/DAP12 receptor complex expressed in microglia, thereby inducing phagocytotic activity 
(Jones et al., 2014). In an AD context however, microglia cannot cope with the increased levels of A $\beta$ oligomers and are functionally impaired (Krabbe et al., 2013). While this impairment might be reversed by reducing the plaque burden, it might induce cytotoxic autoimmunity and thus promote neurodegeneration (Butovsky et al., 2005). Interestingly, upregulation of TREM2 was found to reinstate microglial function thereby reducing plaque burden and rescuing cognitive functions in mice (Jiang et al., 2014). Along with microglia, astrocytes were shown to induce compensatory mechanisms in response to amyloid deposits. Astrocytes bear anti-oxidative functions and show an increased activity near amyloid plaques. Increased levels of $A \beta_{1-42}$ coincide with an induction of oxidative stress which can lead to the activation of caspases driving cell death and thereby promote neurodegeneration (Butterfield et al., 2013; García-Matas et al., 2010).

One potential therapeutic approach might be to prevent the formation of amyloid plaques. While this can be achieved by immunizing individuals with $\mathrm{A} \beta_{1-42}$, clinical trials were aborted due to a pathological induction of CNS inflammatory response upon vaccination (Bard et al., 2000; DeMattos et al., 2001; Hardy, 2002; Schenk et al., 1999). Leinenga and Götz (2015) recently published a non-invasive approach to clear the CNS from amyloid plaques and could successfully reinstate memory function in a transgenic mouse model for AD. Still, further knowledge is needed to understand the molecular processes underlying amyloid pathology in $\mathrm{AD}$ patients.

\subsection{3 tau pathology: neurofibrillary tangles}

The tau protein responsible for the formation of the fibrillary tangles identified by Alois Alzheimer was first described in 1975 (Weingarten et al., 1975). However, it took nine more years until increased protein levels of tau were found in the cerebrospinal fluid of AD patients, given the first implication of that particular protein in the disease (Chapel et al., 1984). Two years later, several research groups identified tau in NFT simultaneously (Grundke-Iqbal et al., 1986; Ihara et al., 1986; Kosik et al., 1986; Wood et al., 1986). The knowledge on tau structure and the function of the respective protein domains is rather little as classical structure analysis are not suitable for proteins like tau (Mukrasch et al., 2009). Probably the most important parts of the tau proteins are the tubulin binding domains and proline-rich domains. In a healthy context, tau stabilizes microtubules thereby promoting their assembly (Brandt and Lee, 1993). Thus, it functions in proliferation and differentiation of cells as well as antero- and retrograde transport 
in neurons (Rodríguez-Martín et al., 2013; Sennvik et al., 2007). The binding towards microtubules is regulated by phosphorylation through cAMP dependent kinases, most likely acting on the proline-rich domains of tau (Landrieu et al., 2006; Mukrasch et al., 2009). Phosphorylation of tau hereby leads to a dissociation of tau from microtubules and thus their destabilization. In human, multiple splice variants of tau are expressed by the MAPT gene (microtubule-associated protein tau) located on chromosome 17 in different developmental stages or cellular compartments (Goedert et al., 1988; Kempf et al., 1996; Wang et al., 1993b). Alternative splicing of MAPT exon 10 results in two isoforms of tau with three (3R) or four repeats (4R) of microtubule-binding domains leading to a significant increase of microtubule polymerization through $4 \mathrm{R}$-tau that is 3 times higher compared to 3R-tau (Goedert et al., 1989).

In AD patients, levels of tau phosphorylation show an up to 4-fold increase (Köpke et al., 1993). This hyperphosphorylation induces a variety of pathological changes. First, hyperphosphorylated tau is unable to bind microtubules and thus to promote their stability and assembly. The resulting disintegration of microtubules is likely to cause axonal degradation and synapse loss which then leads to neuronal cell death and induction of microglia activity (Alonso et al., 1996; Yoshiyama et al., 2007). Second, excessive phosphorylation of tau causes tau proteins to form NFT preceeded by soluble aggregates of tau (see figure 1.6, p.11) (Götz and Ittner, 2008; Ihara et al., 1986). Interestingly, approximately $40 \%$ of all hyperphosphorylated tau proteins remain soluble and do not form fibrils in the brains of AD patients (Köpke et al., 1993). As for amyloid plaques, the mode of action of NFT and soluble tau aggregates is still debated. The classical view on protein aggregates and deposits assumes a pathogenic function and in fact, both soluble tau aggregates and NFT were shown to cause neurodegeneration (Fox et al., 2011; Rohn et al., 2001). Interestingly, the ratio of 3R:4R tau isoforms was found to be shifted towards $4 \mathrm{R}$ in $\mathrm{AD}$ patients (Ginsberg et al., 2006). The increase of $4 \mathrm{R}$ tau might resemble a compensatory response towards hyperphosphorylation of tau, since $4 \mathrm{R}$ tau has a higher microtubule-stabilizing activity (Goedert et al., 1989). However, Chen et al. (2010) found a decrease of neuronal survival and an induction of apoptosis on a gene expression level upon upregulation of $4 \mathrm{R}$ tau isoforms rather indicating a cytotoxic activity.

In contrast to the described implications of tau in neurodegeneration, some researchers question the "tau-hypothesis" of AD pathology since neurodegeneration in AD exceeds NFT burden and 


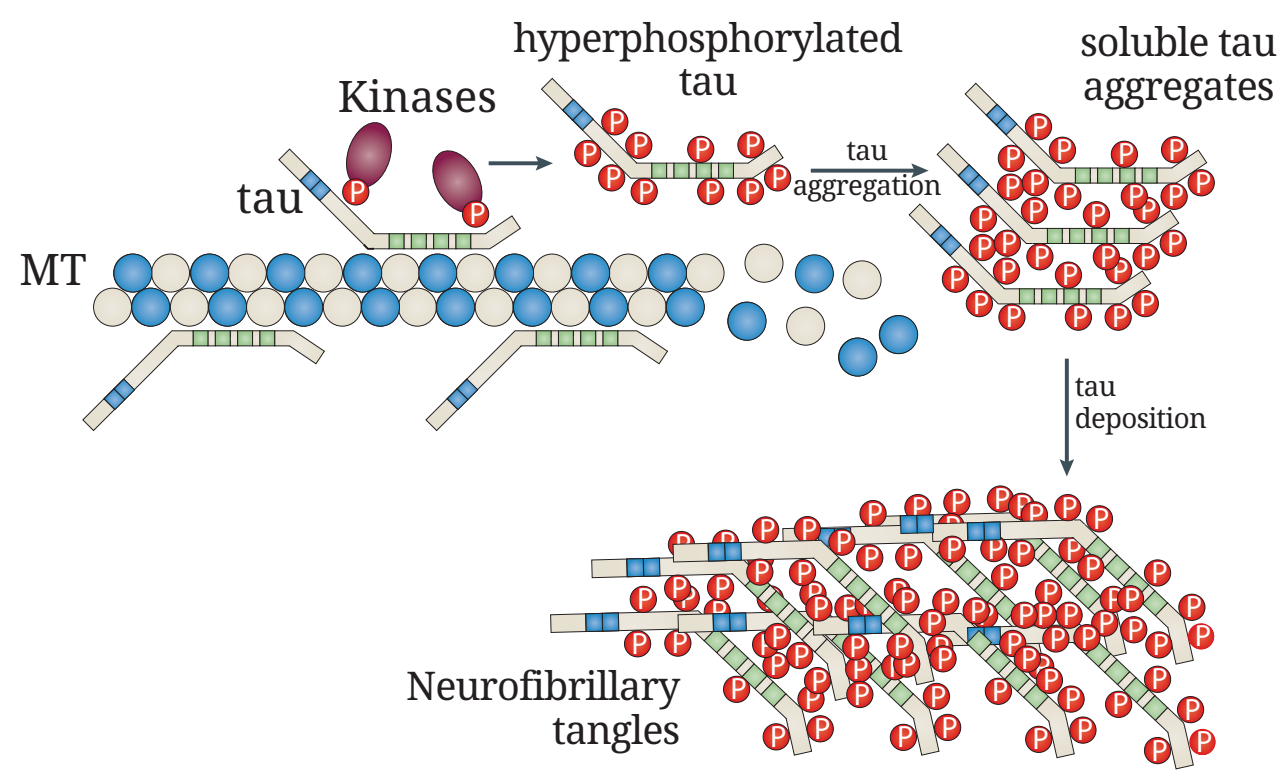

Figure 1.6 - Hyperphosphorylated tau aggregates and forms neurofibrillary tangles:

Microtubuli (MT) are degrading upon dissocation of tau following hyperphosphorylation (P). Graphic was adapted from Götz and Ittner (2008).

neurons containing those fibrils can survive for years and rather propose neuroprotective functions of tau (Gómez-Isla et al., 1997; Lee et al., 2005; Morsch et al., 1999). In general though, the significant function of tau in AD is well accepted and the post-mortem evaluation of AD pathology is commonly based on NFT burden. Braak and Braak defined six stages for tau pathology (see figure 1.7, p.12). In stage I, only a modest number of NFT can be found in few brain regions including the transentorhinal region, the CA1 region, the deeper layers of the entorhinal cortex, and parts of the thalamus and basal forebrain. These modest changes are aggravated during stage II and additional plaques can be found in the subiculum and the association areas of the cerebral cortex.

Stage III is characterized by a severe NFT burden including the first occurrence of "ghost tangles”, extracellular NFT, in the transentorhinal and entorhinal cortex. The hippocampal formation remains rather mildly affected and the isocortex does only rarely show any NFT during that stage. Notably, most AD cases are not diagnosed earlier than Braak and Braak stage III. In stage IV, all previously affected regions do now show severe NFT while the isocortex remains mildly affected. As soon as the isocortex is significantly affected by tau deposits, the tissue is categorized as stage V. The transentorhinal and entorhinal cortex are flooded with ghost tangles and NFT can 
A
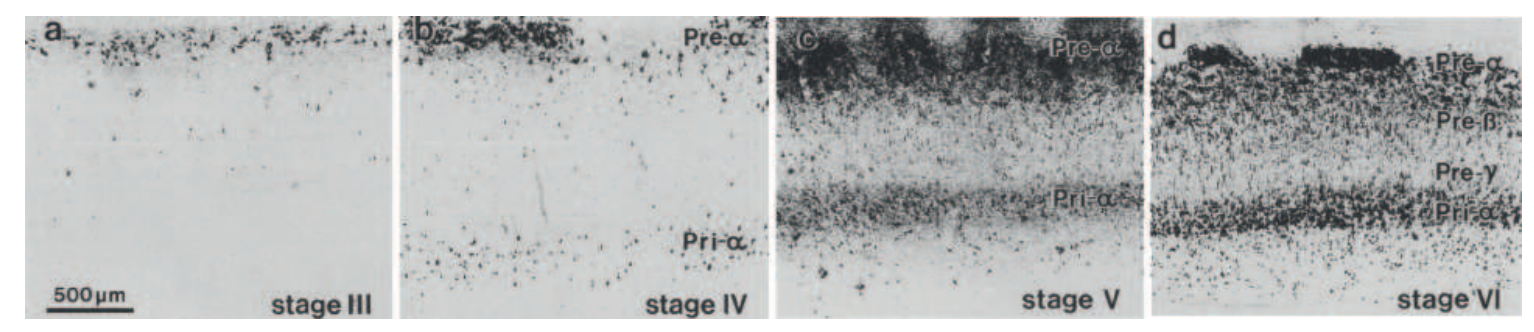

B

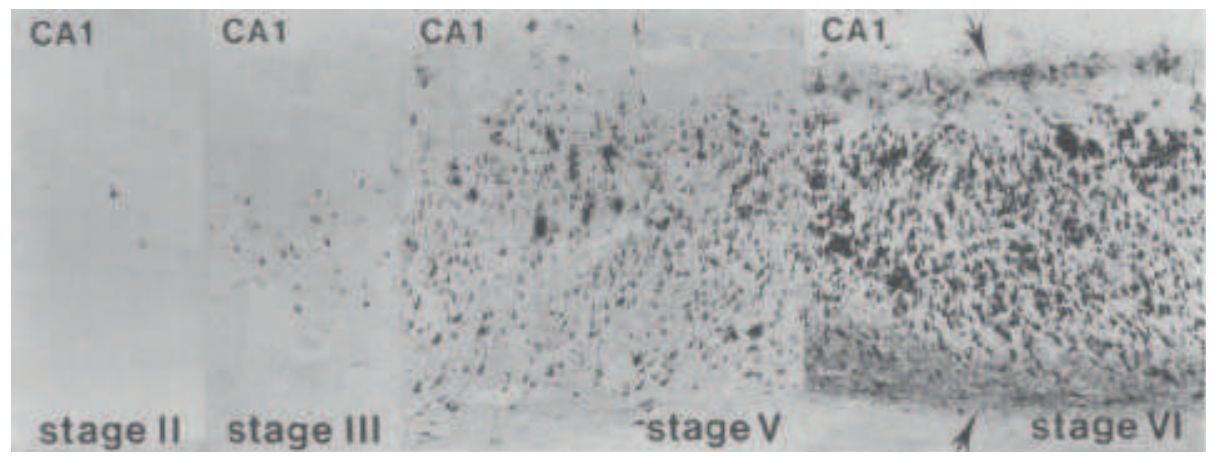

Figure 1.7 - Braak and Braak stages for neurofibrillary tangles:

A) NFT in the different layers of the entorhinal cortex in stage III, IV, V, and VI.

B) Tau pathology in the CA1 in stage II, III, V, and VI. Arrows depict particular dense NFT at the boundaries of the CA1. Unedited graphics were taken from Braak and Braak (1991).

be found all over the hippocampus. Stage VI describes an even more pronounced pathology as found in stage $\mathrm{V}$ brains with a significant loss of neurons and even degradation of ghost tangles within the entorhinal cortex and the hippocampus. NFT are found in all areas of the isocortex including the motor and sensory cortex.

\subsubsection{Risk factors for Alzheimer's disease}

Treatment of $\mathrm{AD}$ patients so far only delays cognitive decline and bears a variety of adverse side effects. Though a number of molecules were proposed as potential drugs for AD, memantine was the last component approved by the FDA for treating AD patients in 2003 (Godyn et al., 2016). The heterogeneity of $\mathrm{AD}$ and the relatively low accuracy of diagnosis prove the discovery of novel drugs to be difficult and, in fact, the number of clinical trials regarding AD is relatively low (Beach et al., 2012; Cummings et al., 2014; Mangialasche et al., 2010). Another approach to counteract $\mathrm{AD}$ besides the treatment of $\mathrm{AD}$ patients is the prevention of its development. This prevention requires a broad knowledge of factors that either increase or decrease the risk for sustaining AD. During the recent decades, knowledge on these risk factors drastically increased 
through epidemiological analysis, supporting pre-clinical studies and potentially reducing future incidence of AD (Barnes and Yaffe, 2011). These risk factors include intrinsic and extrinsic parameters and, unfortunately thus, not all of them can be actively avoided.

One example for these intrinsic factors is the genetic background of an individual. Mutations in a variety of genes were shown to increase the risk for developing AD or even cause a heritable, early-onset form of Alzheimer's disease (Tilley et al., 1998). The first genetic risk factors causing this familial AD were point mutations at APP codons 670/671 (called "Swedish” mutation), 692 ("Flemish”), 693 (”Dutch”), and 717 ("London”) (Goate et al., 1991; Hendriks et al., 1992; Levy et al., 1990; Mullan et al., 1992). During the recent decades, more than 20 additional pathogenic mutations were discovered within the APP gene, a bit less in PS2 and, remarkably, over 200 mutations in PS1 (ALZFORUM, 2016). Interestingly, some mutations of APP might also have neuroprotective functions (Jonsson et al., 2012). While mutations in MAPT can cause a familial form of frontotemporal dementia, there is no evidence yet for a role of genetic tau variants in familial AD (Wolfe, 2009). Familial AD, however, resembles less than 5\% of all AD cases and apart from mutations in APP, PS1 and PS2, more than 20 genetic predispositions were identified increasing the risk for sporadic, late-onset AD (Association, 2015; Van Cauwenberghe et al., 2015). These predispositions include mutations in apolipoprotein E, Trem2, or protein tyrosine kinase 2 beta (Ptk2b) (Jonsson et al., 2013; Strittmatter et al., 1993). Other intrinsic risk factors for AD are female gender and, most importantly, aging (see figure 1.2A, p.2) (Launer et al., 1999).

Apart of the intrinsic risk factors, epidemiological studies identified a broad variety of external impacts on the risk of sustaining AD. In general, researchers found clear correlations between a "healthy lifestyle" and a reduced disease risk. Both physical and cognitive activity, thus education, have protective effects in regard to AD (Barnes and Yaffe, 2011; Sando et al., 2008; Tyas et al., 2001). In contrast, obesity can increase the probability to develop the disease by up to $60 \%$ while regular smoking might even double the risk for AD (Cataldo et al., 2010; Ott et al., 1998; Profenno et al., 2010). In accordance with findings on obesity, patients with diabetes also face an increased probability to develop AD in their later life (Profenno et al., 2010; Tyas et al., 2001). A drastic increase of relative risk was found in individuals that were regularly exposed to defoliants and fumigants or other toxins. Interestingly, any kind of vaccination was shown to coincide with a 2.5 fold reduction of risk which is most likely coherent with a generally healthier 
way of life (Tyas et al., 2001). Besides metabolic diseases like diabetes, other medical conditions are implicated in the development of $\mathrm{AD}$ as well. Patients with migraine or antecedent suffering from post-traumatic stress disorder are more likely to suffer from AD in their later life (Tyas et al., 2001; Yaffe et al., 2010). Altogether, a "healthy lifestyle" together with a good education and avoidance of danger can be considered a promising strategy to reduce one's own individual risk for sustaining AD significantly.

\subsubsection{The APP/PS1 mice and other mouse models for Alzheimer's disease}

Compared to diseases acting on the peripheral nervous system or other human organs than the brain, researchers face a substantial issue when studying CNS diseases. While it is possible for most organs to directly study fresh tissue from living patients by taking biopsies, research on the human brain almost completely depends on indirect sampling (i.e. cerebrospinal fluid) or post-mortem tissue. To circumvent these limitations, researchers commonly use animal models for studying basal functions of the brain or CNS diseases. A variety of genetically modified mouse models for AD were generated during the past 25 years. In 1991, the first transgenic mouse model for AD overexpressing a C-terminal fragment of APP was described 4 years after the discovery of the APP gene (Kang et al., 1987; Kawabata et al., 1991). The first mouse model for tauopathy followed in 1995 (Götz et al., 1995). Nowadays, the most frequently used mouse models for AD either express native or mutated human tau, human APP or APP-fragments, or coexpress human APP and human PS1 driven by PDGF-B-, Thy1-, or Prp-promoters (Hall and Roberson, 2012). Another interesting AD model is the Ck-p25 mouse which features an inducible overexpression of p25 (a truncated form of cyclin-dependent kinase 5 activator) and subsequent inactivation of the cyclin-dependent kinase 5 (CDK5) leading to the formation of tauopathology, neurodegeneration, and cognitive decline (Cruz et al., 2003; Fischer et al., 2005).

The transgenic mouse strain B6-Tg(Thy1-APPswe; Thy1-PS1 L166P) (subsequently called "APP/PS1 mice”) was generated by Radde et al. in 2006. Those mice coexpress human APP carrying the "Swedish mutation" and human PS1 with a single point mutation at codon 166 (CTT to CCT) leading to an exchange of leucine with proline. The "Swedish mutation" of APP describes a double point mutation of codons 670 and 671 (AAGATG to AATCTG) leading to an exchange of 
lysine-methionine with asparagine-leucine (Mullan et al., 1992). The human APP shows a threefold overexpression compared to the endogenous murine APP and as a result, A $\beta$ levels gradually increase drastically during aging (Radde et al., 2006). The first amyloid plaques can be observed at an age of 2 months in the frontal cortex and at 3 months within the hippocampus inducing immune response. Radde et al. evaluated the spatial memory performance of this transgenic mouse line and detected memory impairments at an age of 8 months. Preliminary data from our lab, however, indicate that the memory in APP/PS1 mice is already impaired at an age of 4 months, nicely correlating with increasing plaque burden.

\subsection{Learning and memory}

Probably one of the most important traits in higher animals is learning and memory. The ability to cognitively store information is essential for an individual's survival. Dudai (2002) defined learning as an induced lasting alteration in behavior upon an individual's experience. With the ability to gain knowledge, individuals are able to remember feeding grounds, shelter or avoid adverse situations, i.e. encountering a predator, thereby drastically improve their chance to survive. Besides the ability to communicate with other individuals, memory can be considered as crucial for the regular social interactions and thus cultural evolution. It is a conventional belief that the remarkable human memory is what differentiates us from other animals. This highlights the strong impact of neurodegenerative disease encompassing cognitive decline on patients and their respective families. Understanding the molecular basics underlying learning and memory is thus also crucial for our knowledge of neurodegenerative diseases.

\subsubsection{Categories of memory}

Memory can classically be classified as explicit (declarative) and implicit (procedural) memory (Tulving et al., 1972). Implicit memory functions rather unconsciously and includes priming, procedural memory (habits and skills), associative and non-associative memory (Kandel, 2013). Priming is a process determining how a certain stimulus is perceived based upon previously achieved knowledge regarding both explicit and implicit memory. As an example, the second word of a pair of words is significantly faster processed when it can be associated to the first one 
(Meyer and Schvaneveldt, 1971). Non-associative memory describes learning processes regarding single stimuli including habituation and sensitization (Kandel, 2013). Habituation leads to a decrease in response upon a continuously reappearing stimulus while sensitization describes a general induction of perception upon receiving an intense stimulus (Kandel and Tauc, 1965; Thompson and Spencer, 1966).

Associative memory describes the theoretical linkage between two initially unrelated events (Dudai, 2002). The principles of associative learning were already studied by Ivan Pavlov who was one of the first Novel laureates for Medicine, which he received in 1904. When repeatedly pairing a so called "unconditioned stimulus" naturally evoking a certain behavior with a "conditioned stimulus" that would not cause similar behavior in time, the conditioned stimulus becomes capable of inducing the behavioral response of the unconditioned stimulus. In the classical conditioning performed by Pavlov himself, a bell (conditioned stimulus) was rung to a dog who received a portion of meat powder (unconditioned stimulus), immediately after making the dog salivate. After repeating this procedure a certain time, Pavlov was able to trigger the dog's salivation simply by ringing that particular bell. Another form of associative behavior is operant (or instrumental) conditioning, where an individual's behavior itself becomes associated with an unconditioned stimulus. This form of memory was first described in 1898 by Edward L. Thorndike. When putting cats into a small, closed box with a door opening upon pulling on a string inside of the box, he observed that with increasing number of trials, cats performed better and better in regard to escaping this box (Thorndike, 1898).

Explicit memory can be described as a conscious form of memory and includes acquired knowledge on personal experiences and autobiographic information (episodic memory), and knowledge on facts (semantic memory). For instance, episodic memory helps us to remember a certain moment we listened to a song while semantic memory stores information on the actual musical notes of that particular song. In contrast, playing that respective song on an instrument upon practice involves procedural, thus implicit memory. Interestingly, episodic memory is not "set in stone” and was shown to be remodeled frequently during remembering (Bartlett, 1995).

Memory is further distinguished by its continuance and capacity limits into short-term memory (STM) and long-term memory (LTM). Even though the exact duration of STM is unknown, it is generally believed to last several seconds to minutes in average while there is no temporal 
limitation for LTM (Kandel, 2013). However, researchers claim that the decay of STM does not depend on time but on a limit of memory capacity (Cowan, 2008; Lewandowsky et al., 2004). This capacity can be described as a limited number of theoretical items that can be stored in STM (Miller, 1956). This limitation was initially estimated to $7 \pm 2$ items, though later studies proposed a STM capacity of only 4-6 items maximally (Miller, 1956; Tulving and Patkau, 1962). Importantly, these items might not only resemble a single stimulus but a combination of stimuli into a larger unit (Cowan et al., 2004). In contrast to the debate in STM limitations, LTM is considered not to have any temporal or quantitative limitations and resembles the whole acquired knowledge of an individual's life-time (Cowan, 2008; Kandel, 2013).

\subsubsection{Brain areas involved in different modes of memory}

A healthy and stable memory function consists of three distinct stages: acquisition, consolidation and retrieval. Acquisition describes the perception of short-term storage of novel information therefore includes sensory processes and STM. The transformation of this novelty, thus STM, into stable knowledge, thus LTM, is termed consolidation while memory retrieval is commonly known as "remembering". Memory can also be classified into subtypes. This classification is in general well accepted and evidence from patients with different neuroanatomical symptoms indicate that distinct brain areas are involved in the respective forms and stages of memory. The most commonly known case is patient H.M. who was suffering from a severe epilepsy in the mid 1950s. In order to ameliorate the epileptic seizures, the hippocampus and adjacent regions of the temporal lobe were bilaterally removed via surgery (Scoville and Milner, 1957). Although this particular treatment did reduce the pathology, it led to a significant loss of memory functions in H.M. Interestingly, H.M. had a relatively stable episodic memory for events before his surgery and could remember new information for several seconds and even minutes, though he completely lacked the ability to store these information for a longer period of time. Despite this anterograde amnesia, H.M. was fully capable of learning new tasks though could not recall doing that certain task ever before. Thus, while implicit memory worked fine as well as the acquisition and retrieval of explicit memory, H.M. specifically lacked the ability to consolidate explicit memory. Findings from H.M. indicated that the hippocampus and temporal lobe are crucial for the formation of new explicit memory. Data from other patients with neuroanatomical disturbances 
and more recent data using novel approaches for real-life imaging in vertebrates could confirm these findings and in addition led to the identification of a number of brain regions involved in certain modes of memory (Kandel, 2013).

A
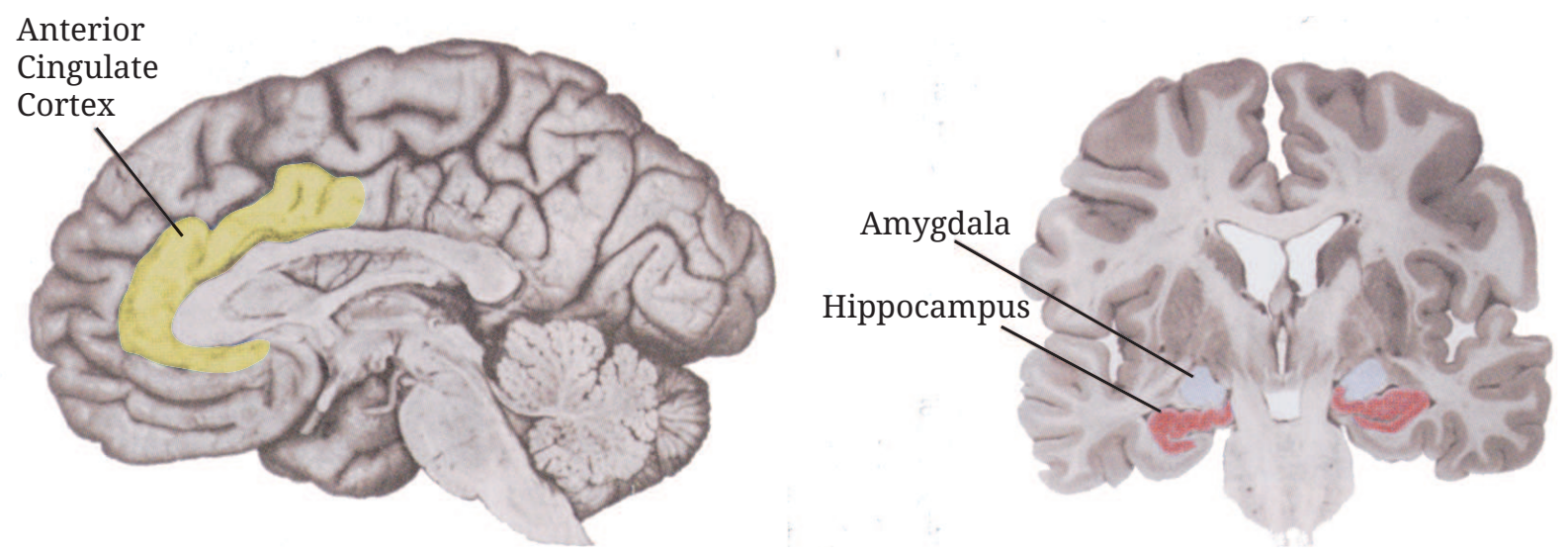

B

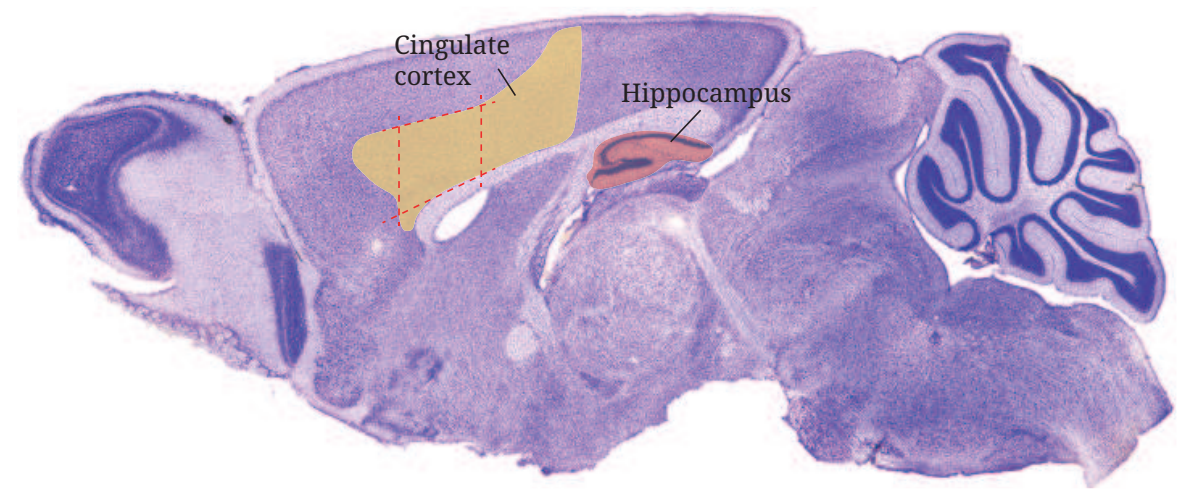

Figure 1.8 - Location of the ACC and hippocampus in human and mouse brains:

A) Sagittal section of a human brain showing the ACC (blue, left) in the frontal lobe below the pre-frontal cortex and frontal sections displaying the hippocampus (red, right) in the medial temporal lobe. Graphics were modified from Kandel (2013).

B) Sagittal sections of a mouse brain showing the cingulate cortex (yellow) and the hippocampus (red). The anterior part of the cingulate cortex (dashed red lines) is considered as the mouse ACC. Graphic modified from Franklin and Paxinos (1997).

The anterior cingulate cortex (ACC) is a part of the frontal lobe located below the pre-frontal cortex and is partially surrounding the corpus callosum in the human and mouse brain alike (see figure 1.8). In human, the ACC shows extensive connections with the hippocampus, amygdala, prefrontal cortex, anterior insula, and the nucleus accumbens indicating its broad impact on cognition but also autonomic functions (Bush et al., 2000; Kandel, 2013). Based on neuroimaging studies and data from patients with lesions inside the ACC, this brain region is most commonly 
linked to error detection and evaluation, reward prediction, decision making, empathy, impulsity and emotion such as aggression (Bubenzer-Busch et al., 2015; Carter et al., 1999; Ende et al., 2016; Lockwood et al., 2015; Nelson et al., 2015; Olié et al., 2015). The anatomical linkage between the ACC and regions involved in memory function like the hippocampus and the pre-frontal cortex, however, indicate its role in memory as well. In fact, several studies could confirm a role of the ACC in learning and memory including the formation of episodic and spatial memory or retrieval of semantic memory (Cazalis et al., 2011; Giannakopoulos et al., 2000; Seo et al., 2015). Further evidence for a role of the ACC in memory function was found by Frankland et al., since gene expression analysis upon contextual fear conditioning and behavior tests following a specific anesthetic inactivation of the ACC clearly demonstrated its importance for associative memory. Disruptions within the ACC were implicated in depression, schizophrenia and bipolar disorder but also in mild cognitive impairment and AD (Giannakopoulos et al., 2000; Kandel, 2013; Nelson et al., 2015; Seo et al., 2015; Tekin et al., 2001).

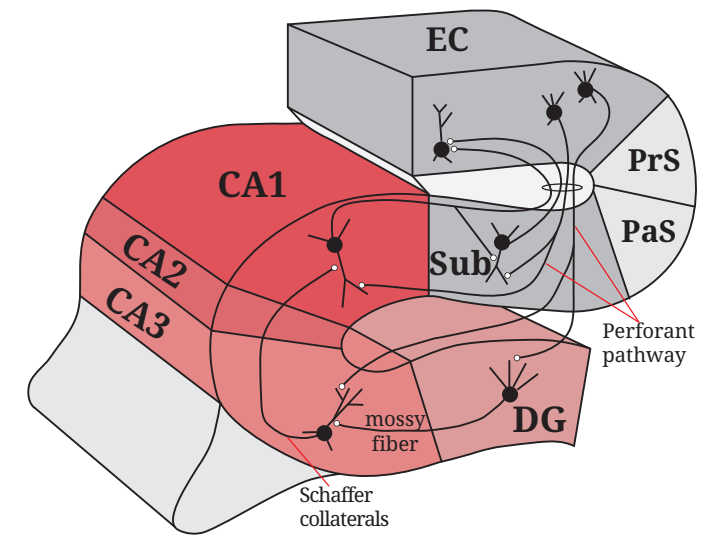

\begin{abstract}
Figure 1.9 - Scheme of the hippocampal formation:

The hippocampal regions (DG, CA3, CA2, CA1) receive their input from the entorhinal cortex (EC) via the perforant pathway. Signaling within the hippocampus is mainly unidirectional through mossy fibers and Schaffer collaterals. Sub: Subiculum; PaS: Parasubiculum; PrS: Presubiculum. Graphic adapted from Kandel (2013).
\end{abstract}

The hippocampal formation, one of the brain regions removed in patient H.M., is located within the medial temporal lobe and reminds of the shape of a seahorse when dissected (see figure 1.8, p.18) (Andersen et al., 2007). It consists of the hippocampal regions cornu ammonis (CA) and dentate gyrus (DG), and the subiculum connecting the hippocampus with the entorhinal cortex via the perforant pathway (see figure 1.9). While the developing hippocampus signals bidirectionally, the adult hippocampus mainly functions unidirectional, signaling from the DG through the different CA regions (Shi et al., 2014). The major input is thereby received by the DG from the entorhinal cortex. The important role of the hippocampus in learning and memory is nowadays well accepted with the respective subregions acting in distinct modes of memory (Battaglia et al., 
2011; Kandel, 2013). The DG is one of the rare sites in the mammal brain where neurogenesis occurs throughout maturity (Gage, 2000). Thereby, neuronal progenitor cells within the subgranular zone of the DG proliferate and mainly differentiate into dentate granule cells (Cameron et al., 1993). This synthesis of new neurons plays an important role in DG-dependent memory, including pattern recognition, temporal separation of acquired memory, or memory remodeling (Aimone et al., 2009; Cao et al., 2004; Deng et al., 2009). This effect on memory function seems to be specific for certain modalities as inhibition of adult neurogenesis impairs some but not all hippocampus related memories (Shors et al., 2002). These memory functions also include spatial memory, associative memory, and explicit sequential learning, strongly depending on the CA1 and CA3 region which are required for both acquisition and retrieval of learned information (Farovik et al., 2010; Montgomery and Buzsáki, 2007; Sakaguchi et al., 2015).

\subsubsection{Cellular mechanisms of learning and memory}

Different forms of memory can be related to certain areas of the brain and thus different neuronal sub-types and networks. It can thus be assumed, that these different forms depend on distinct cellular mechanisms and it is well accepted that STM is in principle regulated through temporal modifications within synapses while LTM depends on alterations in gene expression and resulting long-lasting cellular changes (Abel and Lattal, 2001; Cowan, 2008; Kandel, 2013). As previously described, STM is limited in its duration and capacity, thus requiring cellular mechanisms which can immediately be activated upon request. Implicit STM is considered to act by changing the pre-synaptic strength.

One of the first studies regarding synaptic strength and habituation were done by Spencer et al. (1966). Following a repetitive stimulus, the input from excitatory interneurons on motor neurons in the spinal cord of cats was decreased while the preceding signaling from sensory neurons was unaffected. After a certain time without that repetitive stimulus, Spencer et al. observed restoration of the excitatory signal, nowadays termed dishabituation. Studies in the seaslug Aplysia californica confirmed that habituation relies on reduced pre-synaptic signaling and thus a decrease of neurotransmitter release (Castellucci and Kandel, 1974). Aplysia has a relatively simple nervous system and large neurons thus enabling locally well defined electrophysiological measurements. Besides habituation and dishabituation, the cellular basics of sensitization 
were also studied in the seaslug already in 1965 (Kandel and Tauc, 1965). When transmitting a short electric shock to an animal, it showed an increased pre-synaptic current in interneurons as a response for other - harmless - stimuli as well. Depending on the strength or frequency of the priming stimulus, this sensitization effect lasted for minutes or even weeks and are thus not restricted to STM. More recent studies indicate that the pre-synaptic induction during sensitization depends on a higher rate of exocytosis following each single action potential (Stevens and Wesseling, 1999).

No limits were discovered yet in regard to duration and capacity of LTM and, conclusively, the cellular mechanisms of LTM can not solely rely on an induced neurotransmitter release (Kandel, 2013). Even though there is evidence for a quantitative change in the readily releasable vesicle pool during long-term potentiation and long-term depression, these changes - including an increased number of available neurotransmitter vesicles - depend on upstream mechanisms and most likely changes in gene expression (Goda and Stevens, 1998; Stanton et al., 2003). Thereby, gene expression changes are indirectly caused by neurotransmitters. For instance, the pre-synaptic release of serotonin, which in Aplysia is relevant for long-term potentiation, leads to an activation of serotonin-receptors and the initiation of a protein cascade (Bailey et al., 1996; Varrault et al., 1991). Binding of serotonin to its receptor leads to an increased level of cAMP which can activate the protein kinase A. Upon activation, protein kinase A can be translocated into the nucleus where it can phosphorylate transcription factors (TFs) like CREB (cAMPresponsive element binding protein) thereby regulating gene expression. The resulting changes of de-novo protein synthesis can then lead to a strengthening or dampening of synapses, i.e. by changes in neurotransmitter availability or receptor density (Goda and Stevens, 1998; Stanton et al., 2003; Stecher et al., 1997), a change in synapse number as a result of synaptogenesis or synaptic turnover (Shen and Ganetzky, 2009; Waites et al., 2005), and neurite outgrowth (Modarresi et al., 2012; Tolwani et al., 2002). During the early $21^{\text {st }}$ century, researchers were able to show that these changes in gene expression upon stimulation of neurons does not depend on TFs alone but on an interaction between TFs and epigenetic processes (Guan et al., 2002). 


\subsection{Epigenetics and its impact on gene expression}

Since the discovery of the DNA structure in the early 1950s and preliminary studies on transformation in bacteria, the concept of the DNA as a carrier for hereditary information is well accepted (Avery et al., 1944; Watson and Crick, 1953) The first estimations concerning the number of genes in the human genome were in the range of millions (Vogel, 1964). However, more recent studies suggest approximately 20,000 protein coding genes, highlighting the misconception of the DNA as a blue-print for invariant gene expression in former times (Clamp et al., 2007). Every somatic cell within the human body does in principle contain the same nucleotide sequence, thus requiring a differential expression of genes to enable the broad variety of cells. In 1972, two separate groups identified proteins in Escherichia coli and Bacillus phage SPO1 which are able to specifically modulate gene expression (Ghosh and Echols, 1972; Wilhelm et al., 1972). These proteins, termed transcription factors, can promote or repress gene expression by directly or indirectly binding DNA. Studies from the past decade strongly suggest, that the function of these TFs at least partially depends on epigenetic mechanisms.

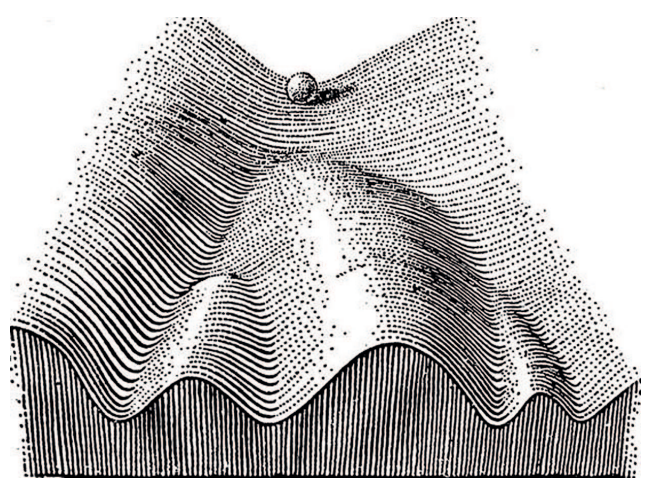

Figure 1.10 - Waddington's "epigenetic landscape": Hypothesis on the modulation of cellular development based on epigenetic regulation of genes. The marble on top of the depicted hill can in principle follow each distinct descent, describing the full genetic potential of each somatic cell. However, epigenetic mechanisms are determining the marble's route, thus the gene expression program. Adapted from Waddington (2014).

The term "epigenetics" originally described causal mechanisms of the development connecting an individual's genotype with its actual phenotype (Waddington, 2012). These mechanisms were later identified as covalent modifications of DNA binding proteins or the DNA itself, but also inhibitory functions of non-coding RNAs on mRNA and are not only happening during development but also in response to several endogenous and exogenous stimuli (Castel and Martienssen, 2013; Jenuwein and Allis, 2001; Robertson et al., 2000). Researchers thus hypothesize that while 
every somatic cell has the full genetic potential, it is the epigenetic machinery that is determining cell state (see figure 1.10, p.22). The methylation of DNA and modifications on histones, DNA binding proteins, is subsequently described in more detail.

\subsubsection{DNA methylation}

DNA methylation was already described in the 1960s and changes in methylation levels were regularly proposed as symptoms of several diseases, including $\mathrm{AD}$, or potential therapeutic approaches for these ever since (Dover et al., 1983; Gold et al., 1963; Pfeifer et al., 1988; West et al., 1995). Methylation of DNA acts mainly within CpG islands of the genome (Robertson et al., 2000). These CpG sites are DNA sequences with a relative frequency of cytosine-guanine dinucleotides higher than 55\%. DNA methylation in human is catalyzed by three distinct DNA methyltransferases (DNMT) by transferring a methyl residue from S-adenosyl methionine to the 5' position of the cytosine pyrimidine ring (Day and Sweatt, 2010) (see figure 1.11, p.24). DNMT1 identifies hemi-methylated CpG-sites and methylates the complementary strand's cytosine (Berkyurek et al., 2014). This process is also called "maintenance methylation”. In contrast, DNMT3A and DNMT3B are also able to catalyze a de-novo methylation of CpG sites (Okano et al., 1999). DNA can be demethylated by 5-methylcytosine demethylases (also termed TET proteins) or DNA glycosylases (Cervoni et al., 1999; Kohli and Zhang, 2013). However, scientists claim that demethylation of cytosine is rather energy-consuming and DNA methylation represents a rather stable epigenetic mechanism (Wolffe et al., 1999).

The development of Next-generation sequencing (NGS) in the past decade allows scientists to sequence DNA in a genome-wide approach, allowing analysis of gene expression via complementary DNA (RNA sequencing), DNA bound by specific proteins (ChIP sequencing) or modified DNA, e.g. methylated DNA (MeDIP sequencing) (Metzker, 2010). In contrast to more classical approaches like gene microarrays, NGS has several advantages which were frequently described in the past years (Marioni et al., 2008; Wang et al., 2014; Zhao et al., 2014). Microarrays are designed to study a pre-defined set of nucleotide sequences and are thus strongly biased while NGS unbiasedly reads sample DNA regardless of the actual sequence, enabling a genome-wide analysis and the identification of novel or exogenous sequences. Additionally, NGS is considered more accurate and replicable than microarray studies. Using this genome-wide approach in regard 


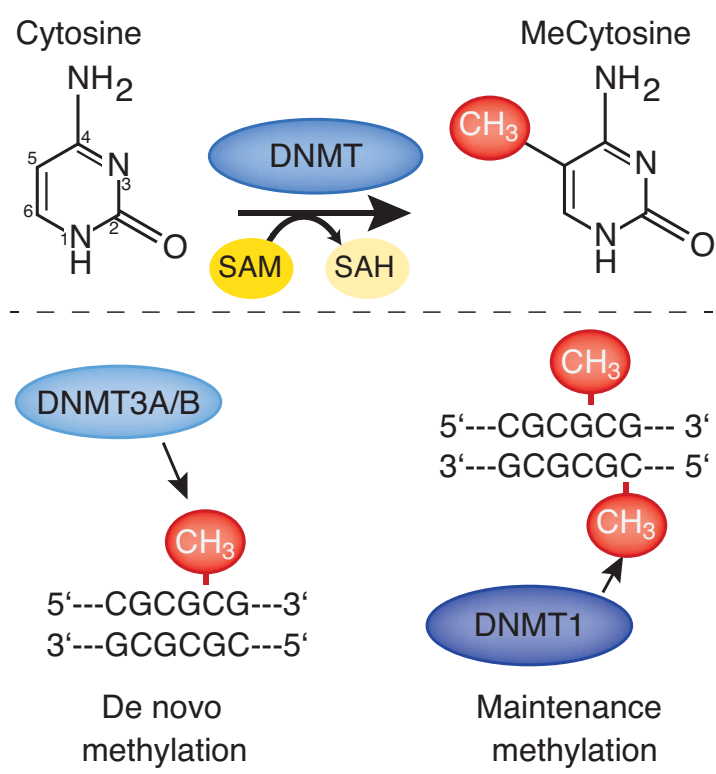

Figure 1.11 - De novo and maintenance methylation of cytosine by DNTMs:

Top: Methylation of cytosine to methylated cytosine (MeCytosine) happens at the 5' position of the pyrimidine ring. DNMTs use S-adenosyl methionine (SAM) as a methyl donor generating methylated cytosine (MeCytosine) and Sadenosyl homocystein (SAH).

Bottom: DNTMs 3A and 3B catalyze de-novo methylation of cystein while DNMT1 methylates the complementary CpG site. Graphic was modified from Day and Sweatt (2010).

to DNA methylation increased our understanding on the function of DNA methylation and its distribution along the genome. DNA methylation in fungi or invertebrates was shown to represent a mosaic pattern where stable methylated regions are interspersed (Suzuki and Bird, 2008). In contrast, MeDIP sequencing of vertebrates revealed a global pattern of large methylation domains primarily in intergenic regions.

In 1981, Mohandas et al. suggested that the suppression of the inactivated x-chromosome in females is driven by DNA methylation as the two x-chromosomes had opposite DNA-methylation patterns. This led to the general understanding of DNA methylation as a mechanism for gene silencing. Recently, Wagner et al. (2014) investigated the correlation between gene expression and DNA methylation state in a non-genome-wide approach. They identified a negative correlation between DNA methylation state at the promoter or gene body and gene expression levels in only $20 \%$ to $30 \%$ of all genes while up to $35 \%$ of genes even showed a positive correlation between DNA methylation and their respective expression level. This was clearly contradictory to the common consensus of DNA methylation causing silencing of genes (Newell-Price et al., 2000). A number of recent genome-wide analysis might indicate, that the negative correlation between DNA methylation and gene expression most likely only accounts for long-term stabilization of gene silencing (Jones, 2012). In addition to gene expression per se, DNA methylation was shown to function in differential splicing as well. Interestingly, alternatively spliced exons 
included during transcription tend to have a higher methylation level than excluded exons and pharmaceutical inhibition of DNA methylation led to an aberrant exclusion of exons (Maunakea et al., 2013). These findings indicate that DNA methylation is not a uniform, binary mechanism but rather variable and multi-functional.

\subsubsection{Histone modifications}

Another epigenetic mechanism is the modification of histones at specific amino acids. Five different histone families are described, namely H1, H2A, H2B, H3, and H4, which mainly function in octamers consisting of two H2A/H2B and two H3/H4 dimers respectively (Bhasin et al., 2006; Luger et al., 1997). The DNA of eukaryotic cells is wrapped around these histone octamers forming a nucleosome, which describes the first magnitude of DNA compaction. Sequences of nucleosomes are condensed once more, forming a structure termed "chromatin" (Allis et al., 2007). Histone 1 acts as a linker protein that strengthens the binding of the histone octamer towards the DNA, maintaining its position inside the nucleosome and regulating a higher-order chromatin structure (Bednar et al., 1998). Modifications on specific residues of these histones were shown to impact the DNA binding properties and promote or repress gene expression (Allis et al., 2007). In addition, some histones within a nucleosome might also be exchanged by an isoform affecting the binding properties as well (Gaume and Torres-Padilla, 2015). These changes on DNA binding and therefore the chromatin structure are one aspect of a process called chromatin remodeling and are considered important mechanisms for regulating gene expression (Allis et al., 2007).

The structure of histones, which consist of a globular and a flexible domain, the "histone tail", is highly conserved among species (Bhasin et al., 2006; Luger et al., 1997). Various covalent modifications on the histone tails were discovered during the past 50 years including acetylation, methylation, phosphorylation, and ubiquitination (Portela and Esteller, 2010). The most prevalent and probably best studied modifications are acetylation (ac) and methylation (me) (see figure 1.12, p.26). For convenience, a terminology for histone modifications was established giving information about the modified Histone, the affected amino acid, and the respective modification. H3K4me3, for instance, resembles tri-methylation of the lysine-residue at the N-terminal position 4 in histone 3 (Allis et al., 2007). 


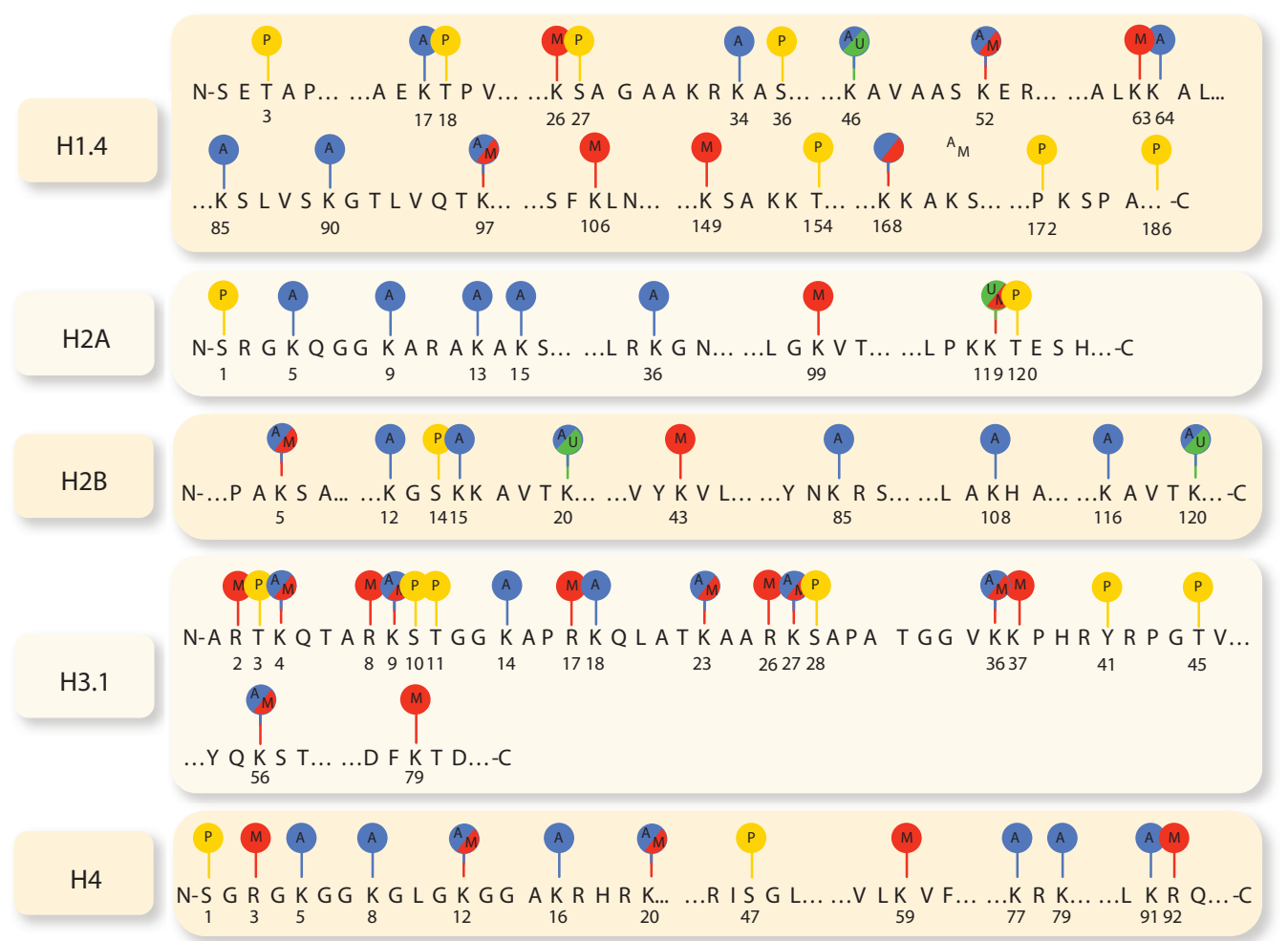

Figure 1.12 - Major modifications of histones H1, H2A, H2B, H3, and H4:

Excerpts of the histone tails' amino acid sequence (single-letter abbreviations) and the major covalent modifications, including acetylation (blue), methylation (red), phosphorylation (yellow), and ubiquitiniation (green). Numbers underneath the respective amino acids resemble their positions within the histone tail. Graphic was modified from Portela and Esteller (2010).

The effects of distinct histone modifications on gene expression are quite diverse and depend on the modified amino acid, respective kind of modification and the position of the modified histone and thus nucleosome within the gene. H3K4me3 for instance is in general implicated with active or poised gene promoters and H3K4me1 with gene enhancers, thus supporting gene expression (Heintzman et al., 2007). In contrast, H3K9me3 mainly occurs within the gene body of repressed genes (Gu et al., 2012; Lachner et al., 2001). Methylation of histone tails is regulated by histone methyltransferases and histone demethylases while acetylation is regulated by histone acetyltransferases and histone deacetylases (HDAC). Interestingly, some of these histone modifying proteins are acting together in protein complexes which usually also contain DNA-binding proteins, ATP-ases and linker-proteins (Hayakawa and Nakayama, 2011). One example is the Mi-2/NuRD complex, containing HDAC1 or HDAC2 along with the lysine-specific demethylase 1 (LSD1) and Mi-2 which functions in nucleosome positioning (Zhang et al., 1997, 1999). Notably, 
histone modifying complexes and histone modifying enzymes themselves were shown to interact with DNA methylation but also TFs. Some histone modifying complexes are able to specifically bind to methylated CpG-sites where they are likely to cause a repression of gene expression (Denslow and Wade, 2007; Hendrich and Bird, 1998).

\subsubsection{DNA methylation and histone modification in age and disease}

Memory impairments are one of the most striking symptoms of AD patients. However, this facet of cognitive decline does also happen during healthy aging, though at milder level (Deary et al., 2009). One potential mechanism driving this progressive decline is the disruption of the nuclear lamina during senescence which was shown to disrupt the three-dimensional organization of chromatin thereby histone modifications and DNA methylation (Berman et al., 2011; Righolt et al., 2011; Shumaker et al., 2006). This pathological effect on epigenetic modifications is thought to trigger severe changes in gene expression observed in AD patients. Another reason for potential disruptions in gene expression is the deregulation of proteins involved in epigenetic mechanisms themselves (Fraga and Esteller, 2007; Kyrylenko et al., 2000). In fact, different patterns of these epigenetic processes can be found in adolescent individuals compared to younger ones and experiments on mice could confirm a linkage between age-dependent memory impairments and altered histone modifications (Fraga and Esteller, 2007; Lubin et al., 2011; Peleg et al., 2010).

The epigenetic profile and, concordantly, gene expression in AD patients is strikingly different than the one from age-matched cognitively healthy human and distinct from changes occurring during healthy aging (Mills et al., 2013; Twine et al., 2011). RNAseq studies on post-mortem tissue from $\mathrm{AD}$ patients revealed that approximately $20 \%$ of all transcripts are differentially expressed in the frontal lobe and parietal cortex compared to samples from healthy controls. The majority of genes were upregulated in their expression in $\mathrm{AD}$ patients. Both up- and downregulated genes could be linked with neuronal functions, cellular metabolsim, and homeostasis. Interestingly, DNA methylation in AD patients also shows a global increase which might resemble a global disruption of the transcription machinery (Bakulski et al., 2012; Coppieters et al., 2014). The APPand MAPT-genes are, in contrast, hypomethylated in some brain areas which is likely to cause a specific induction of APP and MAPT expression (Bakulski et al., 2012; Iwata et al., 2014; West et al., 1995). Some studies performed in mice regarding the specific effect of amyloid-pathology 
on DNA methylation were published recently though their results are rather inconsistent (Cong et al., 2014; Sung et al., 2011). Unfortunately, data concerning histone modifications in AD patients are rather sparse while a considerable amount of studies were conducted in AD mouse models (Fischer, 2014). Results from animal experiments, however, strongly suggest a disruption of histone modifications following amyloid plaque formation (Francis et al., 2009).

Targeting enzymes involved in epigenetic mechanisms is considered a promising approach for counteracting and reinstating memory functions in AD patients (Adwan and Zawia, 2013; Fischer et al., 2010). In fact, a variety of molecules are available targeting DNMTs or histone modifying enzymes like HDACs. The synthetic nucleoside 5-acacytidin is able to inhibit DNMTs, thus DNA methylation (Tanaka et al., 1980). It was approved by the FDA in 2004 for treating myelodysplastic syndromes, though pre-clinical trials in mouse models indicate a beneficial role in reinstating a healthy gene expression in context of AD as well (Sung et al., 2011). One histone modification, namely acetylation, is of particular interest due to the availability of different HDAC-inhibitors, including SAHA (suberoylanilide hydroxamic acid), trichostatin-A, or valproic acid. By inhibiting HDACs Peleg et al. (2010) were able to normalize histone acetylation which is significantly decreased in adolescent mice, thereby reinstating memory functions. Additionally, all three previously mentioned HDAC-inhibitors were able to augment cognitive processes in an APP-mouse model for AD, emphasizing their potential for treating AD patients (Benito et al., 2015; Kilgore et al., 2010). 


\section{Objectives}

The incidence of Alzheimer's disease is prospected to increase dramatically during the next years. However, the possibilities to treat AD patients is strongly limited due to the weak effectiveness of approved drugs and our current inability to actually cure AD. The drug development for $\mathrm{AD}$ is considered problematic due to the heterogeneity of the disease and our relatively little knowledge on the molecular basics involved in its progression and development. In addition, studies to discover novel drugs are often targeting single proteins known to be directly involved in the pathology, i.e. $\gamma$-secretases generating neurotoxic $A \beta$, and are thus strongly biased and restricted to these already known effectors. Recently, HDAC-inhibitors that were initially tested successfully in regard to different types of cancer showed beneficial effects in mouse models for $\mathrm{AD}$ and might be promising treatments for AD patients as well.

I hypothesized that this "pan"-disease efficacy is not exclusive for HDAC-inhibitors thus other, even commonly used drugs might be efficient for $\mathrm{AD}$ as well, and that these drugs can be identified by a molecular-symptomatic (i.e. gene expression or epigenetic changes) approach. In order to test this hypothesis, I aimed to

1. investigate transcriptional and epigenetic genome wide changes specifically caused by amyloid deposition in a longitudinal approach using a mouse mouse model for AD to advance the knwoledge on molecular changes during disease development and progression,

2. identify upstream core modulators - proteins which disruption might cause the deregulation of a variety of downstream cellular processes - so far not linked to $\mathrm{AD}$, and

3. screen public databases to discover and potentially validate new drugs for $\mathrm{AD}$ in the given mouse model, which were not yet considered as promising treatments. 


\section{Materials and Methods}

\subsection{Animals}

All wildtype and transgenic mice used for this study were from the APP/PS1 mouse strain B6Tg(Thy1-APPswe; Thy1-PS1 L166P) (Radde et al., 2006), initially received from Mathias Jucker (German Center for Neurodegenerative Diseases; Tübingen, Germany). Transgenic mice were heterozygously positive for both human APP carrying the swedish mutation (Mullan et al., 1992) and human PS1 with a point mutation at position 166 (leucine to proline) (Moehlmann et al., 2002). Mice from three age groups were chosen for experiments: 1.5 (healthy; young), 4 (early AD pathology; mid-age) and 8 months old (late AD pathology; old)

All mice were bred in groups with their respective littermates, kept in individually ventilated cages $(365 \times 207 x 140 \mathrm{~mm})$ unless otherwise stated. Animals were exposed to a 12 hour light/darkcycle, a stable room temperature (RT) of $22^{\circ} \mathrm{C}$ and air humidity of $58 \%$. Food and water were provided ad libitum.

Housing, breeding and animal experiments were planned and conducted in full compliance with the German Federal Act on the Protection of Animals and were approved by the responsible ministry of Lower Saxony ("Niedersächsisches Landesamt für Verbraucherschutz und Lebensmittelsicherheit”).

\subsubsection{Genotyping}

Mouse tail biopsies were lysed by incubation in $200 \mu$ l DirectPCR tail-lysis buffer (Viagen Biotech;

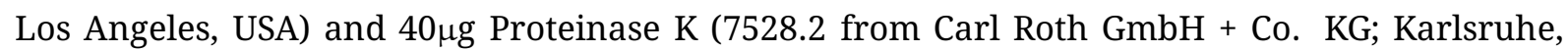
Germany) in a thermoshaker at $1400 \mathrm{rpm}$ and $55^{\circ} \mathrm{C}$ for $3 \mathrm{hr}$ following $85^{\circ} \mathrm{C}$ for $45 \mathrm{~min}$.

The PCR was done with primers for both human APP (3': GAATTCCGACATGACTCAGG, 5': GTTCTGCTGCATCTTGGACA, amplicon size: 264bp) and human PS1 (5': AATAGAGAACGGCAGGAGCA, 3':GCCATGAGGGCACTAATCAT, amplicon size: 608bp) using DreamTaq polymerase (Thermo 
Fisher Scientific; Waltham, USA) according to table 3.1. Samples were evaluated via gel electrophoresis on a 1.5 agarose gel containing $0.1 \%$ ethidiumbromide.

Table 3.1 - Standard protocol for a genotyping PCR using DreamTaq polymerase

\begin{tabular}{|l|c|}
\hline Component & Volume \\
\hline 10x DreamTaq Green Buffer & $2.5 \mu \mathrm{l}$ \\
\hline dNTP Mix, 2.5mM each & $2 \mu \mathrm{l}$ \\
\hline Primer mix, 10mM each & $0.5 \mu \mathrm{l}$ \\
\hline DreamTaq Polymerase & $0.125 \mu \mathrm{l}$ \\
\hline $\mathrm{H}_{2} \mathrm{O}$ & $18.375 \mu \mathrm{l}$ \\
\hline DNA sample & $2 \mu \mathrm{l}$ \\
\hline
\end{tabular}

\begin{tabular}{|l|c|c|}
\hline Step & Temperature & Duration \\
\hline pre-incubation & $95^{\circ} \mathrm{C}$ & $3 \mathrm{~min}$ \\
\hline \multirow{3}{*}{$\begin{array}{l}\text { amplification } \\
30 \mathrm{x}\end{array}$} & $95^{\circ} \mathrm{C}$ & $30 \mathrm{~s}$ \\
\cline { 2 - 3 } & $58^{\circ} \mathrm{C}$ & $60 \mathrm{~s}$ \\
\cline { 2 - 3 } & $72^{\circ} \mathrm{C}$ & $1 \mathrm{~min}$ \\
\hline final elongation & $72^{\circ} \mathrm{C}$ & $5 \mathrm{~min}$ \\
\hline cooling & $4^{\circ} \mathrm{C}$ & $\infty$ \\
\hline
\end{tabular}

\subsubsection{Brain dissection}

To dissect brains, mice were sacrificed by cervical dislocation and decapitated. The scalp was carefully cut open to expose the skull. The posterior and lateral muscles were cut through to ease isolating the brain. The occipital, temporal, and parietal bones were removed after fracturing the interparietal bone by a precise but thorough cut. A transversal incision of the frontal bone allowed removing the brain, which was immediately placed on an ice cold, sterile metal plate for further isolation of brain regions.

\subsubsection{Dissection of the anterior cingulate cortex}

The olfactory bulbs and the most anterior $0.5 \mathrm{~mm}$ of the forebrain were removed using a scalpel. A second coronal cut $3 \mathrm{~mm}$ posterior to the latter one was done to receive a brain slice containing the ACC which is usually visible as a darker triangular region dorsal of the corpus callosum.

\subsubsection{Dissection of the hippocampus}

A superficial cut along the midline of the forebrain was done. The forebrain hemispheres were dissected and freed from the thalamus using a surgical scoop. The hippocampus was now freed from vessels and meninges. The DG was then dissected from the hippocampus by performing 
two orthogonal cuts along its border using a cannula (1.2x50mm, 18G). The remaining hippocampal parts were pulled off the cortex using a surgical scoop and split into CA1 and CA3. All steps starting from the dissected hemispheres were done in ice cold PBS using a stereoscope.

\subsubsection{Intracardial perfusion}

Animals were anesthetized with $30 \mu \mathrm{l} / \mathrm{g}$ avertin and fixed by pinning their limbs once they did not respond to external stimuli anymore. The abdominal cavity was opened with a transversal cut following a careful medial cut to the throat without damaging the rips. The liver was gently pushed aside before removing the thoracic diaphragm. Afterwards, the rips were disjointed laterally without penetrating the lungs.

The heart was freed from the pericardium, a butterfly cannula (Venofix A G21, B.Braun; Melsungen, Germany) connected to a perfusion pump was inserted into the left ventricle and its position was fixed. A precise cut into the right atrium was done to open the circuitry system which was immediately rinsed with $5 \mathrm{ml} / \mathrm{min}$ PBS afterwards. Once the circuitry system was cleared from blood, the tissue was fixated using a total volume of $25 \mathrm{ml}$ ice cold fixation buffer (PBS containing $4 \%$ PFA).

The brain was carefully dissected as previously described (see section 3.1.2, p.31) and incubated in fixation buffer for 6 hours. Afterwards it was transferred to $30 \%$ sucrose in water and incubated for one day before it was dried, sealed in aluminum foil and frozen in liquid nitrogen.

The used anesthetic Avertin was produced by slowly adding $71 \mathrm{ml}$ distilled water to a mixture of 1g 2,2,2-tribromoethanol and $1 \mathrm{ml} 98 \%$ isoamylalcohol. 


\subsection{Molecular analysis}

\subsubsection{Immunohistochemistry}

For immunohistochemistry, mouse brains were cut into slices using a cryostat (Leica; Wetzlar, Germany) set to $-20^{\circ} \mathrm{C}$. Brains were allowed to acclimate for at least $25 \mathrm{~min}$ before sectioning and fixation. For hippocampal slices, brains were sectioned by cutting coronally $\approx 0.5 \mathrm{~mm}$ and $3.3 \mathrm{~mm}$ posterior to the Bregma. Sections were placed on a specimen stage covered with Jung tissue freezing medium (Leica; Wetzlar, Germany) with the posterior plane facing down. They were then sealed with the freezing medium. After letting the medium dry for $20 \mathrm{~min}$, the sections were cut into $40 \mu \mathrm{m}$ thick slices which were transferred to 24 well plates. Each well was previously

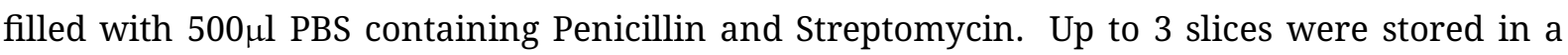
single well.

Table 3.2 - Primary (top) and secondary (bottom) antibodies for immunohistochemistry

\begin{tabular}{|c|c|c|l|l|}
\hline Target protein & Host species & Concentration & vendor \& product ID \\
\hline NeuN & guinea pig & $1: 1000$ & Synaptic Systems (Göttingen, Germany) - 266-004 \\
\hline GFAP & rabbit & $1: 1000$ & Abcam (Cambridge, UK) ab7260 \\
\hline IBA1 & rabbit & $1: 1000$ & Wako Chemicals (Neuss, Germany) 019-19741 \\
\hline human A $\beta$ & rabbit & $1: 1000$ & Tecan (Männedorf, Switzerland) JP18584 \\
\hline Target protein & Dye & Host species & Concentration & vendor \& product ID \\
\hline rabbit IgG & AlexaFluor 488 & goat & $1: 1000$ & $\begin{array}{l}\text { Life technologies (Carlsbad, USA) } \\
\text { A11034 }\end{array}$ \\
\hline guinea pig IgG & AlexaFluor 633 & goat & $1: 1000$ & $\begin{array}{l}\text { Life technologies (Carlsbad, USA) } \\
\text { A21105 }\end{array}$ \\
\hline
\end{tabular}

Brain slices of the same anteroposterior region were selected for each sample and transferred to a fresh 24 well plate. The slices were incubated twice in PBS containing $0.2 \%$ Triton-X to permeabilize cell membranes. Samples were blocked afterwards with 5\% normal goat serum in PBS containing $0.3 \%$ Triton-X for $1.5 \mathrm{hr}$. The blocking medium was removed and primary antibodies dissolved in $300 \mu \mathrm{l}$ PBS containing 1\% normal goat serum and $0.3 \%$ Triton-X were added and incubated at $4^{\circ} \mathrm{C}$ over night (see table 3.2 top panel). Next, samples were washed thrice for 10 minutes with PBS containing 1\% normal goat serum. Afterwards, secondary antibodies were 
added dissolved in 300 $\mu$ PBS containing 1\% normal goat serum and 0.3\% Triton-X and incubated for $2 \mathrm{hr}$ (see table 3.2 bottom panel, p.33). Samples were washed twice with PBS for $5 \mathrm{~min}$ before incubating with DAPI (6335.1 from Carl Roth GmbH + Co. KG; Karlsruhe, Germany) (1:10,000) in PBS for 20min. The samples were finally washed once more with PBS for $5 \mathrm{~min}$ and mounted on objective slides using mowiol (Sigma Aldrich; St. Louis, USA). Mounted slides were dried briefly at $37^{\circ} \mathrm{C}$ for $20 \mathrm{~min}$ or over night at $4^{\circ} \mathrm{C}$.

\subsubsection{Confocal imaging}

Confocal imaging was done with a ZEISS LSM 710 (Carl Zeiss AG; Oberkochen, Germany) equipped with a 20x objective. Images were acquired using the ZEN lite software from ZEISS with a resolution of $2048 \times 2048$ pixel at $1.58 \mu$ s/pixel, bit depth of 12 Bit and 8 times line averaging. Laser and software settings for image acquisition were set up once prior to image acquisition and remained unchanged during the experiment.

\subsubsection{RNA isolation}

RNA isolation was performed in a sterile fume hood and all technical equipment used was previously washed with 70\% ethanol and RNAseZap to avoid contamination and degradation of RNA. Brain samples were kept on dry ice until they were processed and all steps were performed on ice unless otherwise stated to reduce degradation. The RNA isolation protocol was adapted from the TRIzol reagent protocol from Thermo Fisher Scientific (Waltham, USA).

Each sample was supplied with $500 \mu \mathrm{l}$ TRIzol-reagent and 10 ceramic beads before homogenizing in an Omni Bead-Ruptor 24 (Omni International; Kennesaw, USA) for 10s at a relative speed of 2.6. Afterwards, the samples were incubated at RT for 5 minutes to ensure a proper lysis of the tissue. $100 \mu \mathrm{l}$ chloroform was added, samples were mixed manually for 15 seconds and incubated for another 5 minutes at RT. After centrifuging at 12,000g for 15 minutes at $4^{\circ} \mathrm{C}$, the transparent upper phase was transferred to a fresh tube. $250 \mu$ isopropanol and $1 \mu$ l GlycoBlue (Thermo Fisher Scientific; Waltham, USA) were added to each sample before they were incubated at $-20^{\circ} \mathrm{C}$ over night to precipitate the RNA. 
Then, the samples were centrifuged at $12,000 \mathrm{~g}$ for 30 minutes at $4^{\circ} \mathrm{C}$ and the supernatant was discarded carefully. For washing, the RNA pellets were mixed with $300 \mu \mathrm{l} 75 \%$ ethanol, vortexed and centrifuged at $12,000 \mathrm{~g}$ for 5 minutes at $4^{\circ} \mathrm{C}$ before removing the supernatant. This washing step was repeated once. Before redissolving the RNA, the tubes were briefly spinned and freed from remaining liquid. Samples were air-dried for 2 minutes to clean the RNA from ethanol. Finally, RNA pellets were redissolved in sequencing graded water (W4502 from Sigma Aldrich; St. Louis, USA) and incubated for 10 minutes at $42^{\circ} \mathrm{C}$ before proceeding with DNAse treatment.

\subsubsection{DNAse treatment}

To ensure a high quality of RNA, samples were treated with DNAse I (EN0525, from Thermo Fisher Scientific; Waltham, USA) to remove any contamination with DNA. Therefore, samples were mixed with reaction solution prepared according to the DNAse I standard protocol from Thermo Fisher Scientific (Waltham, USA), described in table 3.3. After incubating at $37^{\circ} \mathrm{C}$ for 20 minutes, $150 \mu$ l sequencing graded water was added to each sample and phenol-chloroform extraction was performed.

Table 3.3 - DNAse I reaction solution.

\begin{tabular}{|l|c|}
\hline Component & Volume \\
\hline $10 \mathrm{x}$ Incubation Buffer & $5 \mu \mathrm{l}$ \\
\hline DNAse I $(10 \mathrm{U} / \mu \mathrm{l})$ & $1 \mu \mathrm{l}$ \\
\hline RNAse OUT $(40 \mathrm{U} / \mu \mathrm{l})$ & $0.5 \mu \mathrm{l}$ \\
\hline RNA in sequencing graded $\mathrm{H}_{2} \mathrm{O}$ & $\mathrm{x} \mu \mathrm{l}$ \\
\hline Sequencing graded $\mathrm{H}_{2} \mathrm{O}$ & $43.5-\mathrm{x} \mu \mathrm{l}$ \\
\hline
\end{tabular}

\subsubsection{Phenol-chloroform extraction}

To retrieve the RNA from the DNAse reaction solution, a phenol-chloroform extraction was done. Each sample was mixed with $200 \mu \mathrm{l}$ phenol/chloroform/isoamyl alcohol (25:24:1) and centrifuged at $13,000 \mathrm{~g}$ for 2 minutes at $4^{\circ} \mathrm{C}$. The aqueous phase was transferred to a fresh tube and mixed with $1 \mu \mathrm{l}$ GlycoBlue (Thermo Fisher Scientific; Waltham, USA), $20 \mu \mathrm{l} 3 \mathrm{M}$ sodium-acetate (pH 4.8), and $200 \mu \mathrm{l}$ isopropanol. Samples were incubated over night to precipitate the RNA and processed 
further as previously described (see section 3.2.3, p.34). RNA content was quantified using a Nanodrop 2000 (Thermo Fisher Scientific; Waltham, USA) and purity was validated using an Agilent 2100 Bioanalyzer (Agilent Technologies; Santa Clara, USA). Samples with a RNA integrity number below 8.0 were re-purified as described.

\subsection{4 cDNA synthesis}

To quantify specific transcripts via quantitative PCR, the RNA first has to be rewritten into cDNA (complementary DNA). To do so, the Transcriptor First Strand cDNA Synthesis Kit (Roche; Basel, Switzerland) was used following the standard protocol. Samples and reagents were kept on ice during the whole procedure unless otherwise stated.

An equal amount of RNA templates was mixed with primers and RNAse-free water according to table 3.4 (top left panel) and denatured by incubating at $65^{\circ} \mathrm{C}$ for 10 minutes in a PCR cycler. Afterwards, the reaction mixture was set up according to table 3.4 (bottom left panel), added to each template-primer mix and incubated as described below (see table 3.4 right panel) in a PCR cycler.

Table 3.4 - Standard protocol for Transcriptor First Strand cDNA Synthesis

\begin{tabular}{|l|c|}
\hline Component & Volume \\
\hline Random hexamer primer $(0.6 \mathrm{mM})$ & $2 \mu \mathrm{l}$ \\
\hline RNA template (500ng) & $\mathrm{x} \mu \mathrm{l}$ \\
\hline RNAse-free $\mathrm{H}_{2} \mathrm{O}$ & $11-\mathrm{x} \mu \mathrm{l}$ \\
\hline
\end{tabular}

\begin{tabular}{|l|c|}
\hline Component & Volume \\
\hline $5 x$ Reaction buffer & $4 \mu \mathrm{l}$ \\
\hline RNAse Inhibitor $(40 \mathrm{U} / \mu \mathrm{l})$ & $0.5 \mu \mathrm{l}$ \\
\hline $\mathrm{dNTP}$ Mix $(10 \mathrm{mM}$ each) & $2 \mu \mathrm{l}$ \\
\hline Reverse Transcriptase $(20 \mathrm{U} / \mu \mathrm{l})$ & $0.5 \mu \mathrm{l}$ \\
\hline template-primer mix & $13 \mu \mathrm{l}$ \\
\hline
\end{tabular}

\begin{tabular}{|c|c|}
\hline Temperature & Incubation time \\
\hline $25^{\circ} \mathrm{C}$ & $10 \mathrm{~min}$ \\
\hline $55^{\circ} \mathrm{C}$ & $30 \mathrm{~min}$ \\
\hline $85^{\circ} \mathrm{C}$ & $5 \mathrm{~min}$ \\
\hline $4^{\circ} \mathrm{C}$ & $\infty$ \\
\hline
\end{tabular}

Upper left: template primer mix. Bottom Left: cDNA synthesis reaction solution. Right: cDNA synthesis reaction protocol. 


\subsubsection{Quantitative real time PCR}

Quantitative real time PCR (qPCR) was used to quantify specific mRNA levels. Primers for qPCR were designed using the "UPL Assay Design Center" (http://www.universalprobelibrary.com) from Roche and ordered from Sigma Aldrich (St. Louis, USA). Samples were mixed with Primers, LightCycler480 MasterMix and a universal probe library and run in a LightCycler 480 (Roche; Basel, Switzerland) as described in table 3.5.

Table 3.5 - Protocol for qPCR of mRNA samples with LightCycler 480

\begin{tabular}{|l|c|}
\hline Component & Volume \\
\hline PCR-graded $\mathrm{H}_{2} \mathrm{O}$ & $4.05 \mu \mathrm{l}$ \\
\hline Primer Mix (10mM each) & $0.3 \mu \mathrm{l}$ \\
\hline LightCycler480 Master Mix & $7.50 \mu \mathrm{l}$ \\
\hline Universial probe library & $0.15 \mu \mathrm{l}$ \\
\hline cDNA sample (1:10 diluted) & $3.00 \mu \mathrm{l}$ \\
\hline
\end{tabular}

\begin{tabular}{|l|c|c|}
\hline Step & Temperature & Duration \\
\hline pre-incubation & $95^{\circ} \mathrm{C}$ & $10 \mathrm{~min}$ \\
\hline \multirow{3}{*}{$\begin{array}{l}\text { amplification } \\
45 \mathrm{x}\end{array}$} & $95^{\circ} \mathrm{C}$ & $10 \mathrm{~s}$ \\
\cline { 2 - 3 } & $60^{\circ} \mathrm{C}$ & $30 \mathrm{~s}$ \\
\cline { 2 - 3 } & $72^{\circ} \mathrm{C}$ & $1 \mathrm{~s}$ \\
\hline cooling & $40^{\circ} \mathrm{C}$ & $30 \mathrm{~s}$ \\
\hline
\end{tabular}

Left: qPCR reaction solution. Right: Light cycler program.

\subsubsection{RNA sequencing}

Messenger RNA from the ACC, CA1 and DG of 1.5, 4 and 8 months old APP/PS1 wildtype and transgenic mice $(n=6)$ was sequenced for genome wide gene expression analysis using a HighSeq 2000 sequencing system (Illumina; San Diego, USA). Paired-end RNA libraries were prepared from the CA1 and single-end libraries from ACC and DG using the TruSeq RNA Library Preparation Kit v2 and subsequently the TruSeq PE Cluster Kit v3-cBot-HS or TruSeq SR Cluster Kit v3-cBot-HS respectively following the standard protocols from Illumina. Quality of RNA libraries was validated using a Nanodrop 8000 (Thermo Fisher Scientific; Waltham, USA), Qubit 2.0 fluorometer (Life technologies; Carlsbad, USA) and RNA 6000 Nano Kit on a Agilent 2100 Bioanalyzer (Agilent Technologies; Santa Clara, USA). 


\subsubsection{Fluorescence-activated cell sorting (FACS) of nuclei}

To perform celltype-specific Chromatin immunoprecipitation (ChIP) and methylated DNA immunoprecipitation, nuclei from the ACC, CA1 and DG of 1.5, 4 and 8 months old APP/PS1 wildtype and transgenic mice were isolated and sorted using fluorescence-activated cell sorting (FACS) as previously described (Bonn et al., 2012). Samples from 3 animals (6 for 1.5 months old mice) per group were pooled to retrieve a feasible amount of chromatin.

The pooled tissue was homogenized in $100 \mu$ l low sucrose buffer using motorized micro-pestles. The pestle was washed in $400 \mu \mathrm{l}$ of the same buffer which was then added to the homogenate. For cross-linking, a final concentration of $1 \%$ formaldehyde was added and incubated for $5 \mathrm{~min}$ at RT on a rotation wheel. The sample was quenched by adding $51.4 \mu \mathrm{l} 1.25 \mathrm{M}$ glycine for another $5 \mathrm{~min}$ at RT. $500 \mu \mathrm{l}$ low sucrose buffer was added. The samples were washed by centrifuging at $2000 \mathrm{~g}$ for $3 \mathrm{~min}$ at $4^{\circ} \mathrm{C}$ and the resulting supernatant was removed carefully. The pellet was dissolved in $1 \mathrm{ml}$ low sucrose buffer and homogenized a second time using a T10 basic Ultra-Turrax (IKA; Staufen im Breisgau, Germany) for 30s at a relative force of 3.5.

Nuclei were isolated using a sucrose gradient. The homogenate was carefully layered onto $6 \mathrm{ml}$ high sucrose buffer in oak ridge tubes and centrifuged at $3200 \mathrm{~g}$ for $10 \mathrm{~min}$ at $4^{\circ} \mathrm{C}$. The buffer was carefully removed without disturbing the nuclei pellet. The pellet was resuspended in $1 \mathrm{ml}$ PBTB and washed by centrifuging at $2000 \mathrm{~g}$ to remove any residual sucrose buffer. The oak ridge tube was rinsed with $750 \mu \mathrm{l}$ PBTB to retrieve any remaining nuclei. The nuclei were collected by centrifuging at $4000 \mathrm{~g}$ for $5 \mathrm{~min}$ at $4^{\circ} \mathrm{C}$ and stained by resuspending the pellet in $300 \mu \mathrm{l}$ PBTB containing 3\% normal goat serum and conjugated anti-NeuN antibody from mouse (1:500) (MAB377 from Merck Millipore; Darmstadt, Germany) for $30 \mathrm{~min}$ at $4^{\circ} \mathrm{C}$ on a rotation wheel. The staining was stopped by washing the nuclei four times as described above.

Before loading a sample into the FACS machine, the samples were pathed through a 26G needle and syringe to detach nuclei and avoid nuclei cluster. Sorting was done in a FACSARIA III (BD Biosciences; Franklin Lakes, USA) and sorted cells were collected in 1.5ml Eppendorf tubes. Tubes for nuclei collection were coated with bovine serum albumin by rinsing them with $300 \mu l$ PBTB for 1 hour at RT prior to sorting to prevent the nuclei from attaching to the tube's wall. Sorted nuclei were pelleted by centrifugation at $20,000 \mathrm{~g}$ for $5 \mathrm{~min}$ at $4^{\circ} \mathrm{C}$, redissolved in $100 \mu \mathrm{l}$ 
RIPA buffer and transferred to $600 \mu$ l Bioruptor tubes.

\subsubsection{Chromatin preparation from sorted nuclei}

Sorted nuclei in RIPA buffer were sheared 4 times for 5 cycles (30s on/off) in a Bioruptor Plus (Diagenode; Liège, Belgium) with high power. After each shearing cycle, the samples were briefly spun down. Afterwards, the samples were centrifuged at $16,000 \mathrm{~g}$ for $5 \mathrm{~min}$ at $4^{\circ} \mathrm{C}$ to separate the sheared chromatin containing supernatant from residual nuclear compartments. All following steps were conducted in DNA LoBind tubes (Eppendorf; Hamburg, Germany).

A proper shearing of chromatin was validated for every single sample by isolating the DNA. Therefore, $2 \mu$ l of each sample was mixed with $8 \mu \mathrm{l}$ elution buffer containing $0.1 \mu \mathrm{g} / \mu \mathrm{l}$ RNAse A and incubated for $45 \mathrm{~min}$ at $37^{\circ} \mathrm{C}$ to reduce the concentration of SDS, elute the DNA and digest residual RNA. $10 \mu \mathrm{l}$ Wienmann shearing buffer containing $2 \mu \mathrm{g} / \mu \mathrm{l}$ proteinase $\mathrm{K}$ was added and samples were incubated over night at $65^{\circ} \mathrm{C}$ in a thermoshaker at $800 \mathrm{rpm} .20 \mu \mathrm{l}$ SureClean along with $3 \mu \mathrm{L}$ LPA as a co-precipitant were added to precipitate the DNA. Samples were rigorously vortexed and centrifuged at 15,000g for 20min after incubating for 10min at RT. The DNA pellets were washed twice with $200 \mu \mathrm{l} 70 \%$ ethanol by centrifuging at $15,000 \mathrm{~g}$ for $5 \mathrm{~min}$. Afterwards, the ethanol was removed and possible remainings were allowed to evaporate. The pellets were dissolved in $15 \mu$ l elution buffer.

DNA content was measured with a Qubit 2.0 fluorometer (Life technologies; Carlsbad, USA) and fragmentation of DNA was quantified using a DNA High Sensitivity Kit on a Agilent 2100 Bioanalyzer (Agilent Technologies; Santa Clara, USA). The desired fragmentation reached from 100500bp with an average fragment size of 300bp. In case the DNA fragments did exceed the given sizes, respective samples were re-sheared and fragmentation as well as DNA content were validated again. 


\subsubsection{Chromatin immunoprecipitation (ChIP)}

ChIP sequencing was performed to investigate potential changes of H3K4 methylation state. Chromatin from neuronal and non-neuronal nuclei retrieved from the ACC and DG of 1.5, 4 and 8 months old APP/PS1 wiltype and transgenic mice ( $\mathrm{n}=2$ each) was immunoprecipitated using an H3K4me3 antibody (ab8580 from Abcam; Cambridge, UK). Each processed sample equaled a pool of at least 3 animals. All steps were done using DNA LoBind tubes (Eppendorf; Hamburg, Germany) to reduce loss of DNA to a minimum.

From each sample, $250 \mu \mathrm{g}$ DNA were diluted 1:10 in IP buffer without SDS to reduce the concentration of SDS and, thus, ensure a proper antibody-binding. Magnetic beads were blocked

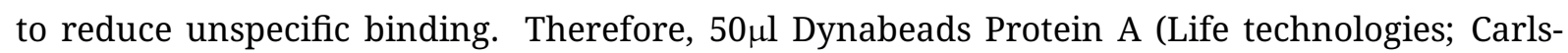
bad, USA) per ChIP sample were washed once with IP buffer and afterwards incubated in IP buffer containing $0.5 \%$ bovine serum albumine for $2 \mathrm{hr}$. The beads were washed twice with $1 \mathrm{ml}$

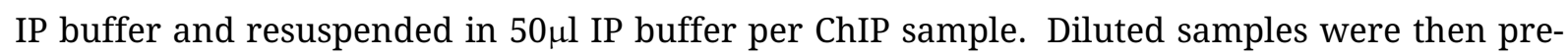
cleared by adding $30 \mu \mathrm{l}$ blocked magnetic beads for $1 \mathrm{hr}$ at $4^{\circ} \mathrm{C}$ to reduce unspecific precipitation of chromatin. Samples were cleaned from beads and $2 \%$ of each sample was taken as an input.

The H3K4me3 antibody was added to each sample and incubated over night at $4^{\circ} \mathrm{C}$ on a rotation wheel. $20 \mu \mathrm{l}$ of blocked magnetic beads were added and the samples were incubated for another $1.5 \mathrm{hr}$ at $4^{\circ} \mathrm{C}$. Samples were washed twice with a sequence of three different buffers $(2 \mathrm{x}$ IP buffer, 3x LiCl buffer, 2x IP buffer, 2x TE buffer) to isolate specifically precipitated chromatin. Afterwards, the DNA of ChIPed and input samples was isolated as described in section 3.2.8 (see p.39).

\subsubsection{Library preparation for Multiplex Illumina ChIP-sequencing}

DNA libraries for ChIP samples were generated using the NEBNext Ultra DNA Library Prep Kit for Illumina, NEBNext Multiplex Oilgos for Illumina (NewEngland Biolabs; Ipswich, USA) and AMPure XP beads (Beckman Coulter; Breas, USA) for end repair and adaptor ligation, amplification and clean-up respectively.

As fragmentation of DNA might lead to blunt ends or 3'- and 5'-overhangs of different size, both 
ends of the fragments need to be converted into blunt ends and tagged by a single adenine nucleotide to avoid fragment-oligomerization. For end repair, DNA samples were mixed with $1.5 \mu \mathrm{l}$ End Prep Enzyme Mix and 1.5 $\mu$ l End Repair Reaction Buffer following incubation for 30min at $20^{\circ} \mathrm{C}$ and $65^{\circ} \mathrm{C}$ respectively. Adapter ligation was done by adding 7.5 $\mu \mathrm{B}$ Blunt/TA Ligase Master Mix, $2 \mu \mathrm{l}$ NEBNext Adapter and 0.5 $\mu$ l NEBNext Ligation Enhancer to each sample followed by incubation for $30 \mathrm{~min}$ at $20^{\circ} \mathrm{C}$. Adapters are specific sequences for 5 ' and 3 ' ends organized in a stem-loop and separated by a central uracil. This structure forces adapters to bind a single DNA fragment exclusively. Uracil is afterwards excised by adding $1.5 \mu \mathrm{l}$ USER enzyme to each sample and incubating for $30 \mathrm{~min}$ at $37^{\circ} \mathrm{C}$.

DNA fragments are then isolated using AMPure XP beads which selectively bind to DNA with a preference for fragments longer than 100bp and, thus, allow size selection of fragments. $26.5 \mu \mathrm{l}$ AMPure XP beads were added to a sample and incubated for $5 \mathrm{~min}$ at RT before removing the supernatant. Beads were washed twice with $200 \mu \mathrm{l} 80 \%$ ethanol and air-dried for 2 minutes. DNA was eluted from the beads by adding $17 \mu$ l elution buffer, vortexing and incubating for $2 \mathrm{~min}$ at RT.

The number of amplification cycles needed for each single ChIP sample was determined via qPCR according to the protocol in table 3.6 (see p.42). The ct value was computed using the LightCycler

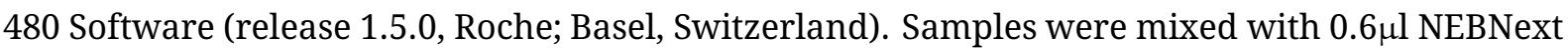

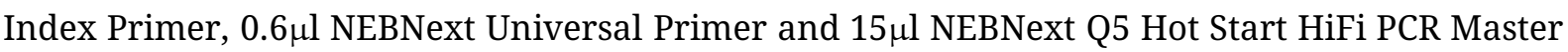
Mix. For multiplex sequencing, samples that were run on the same lane need to be amplified with different indices. Six samples were run per lane, thus, index primes 2, 4, 5, 6, 7 and 12 were used for amplification as those share the least similarity among each other. Samples were amplified for ct-2 PCR cycles according to table 3.6 (see p.42).

The DNA was isolated as described and transferred to a DNA LoBind tube (Eppendorf; Hamburg, Germany). DNA content was measured with Qubit 2.0 fluorometer (Life technologies; Carlsbad, USA) and quality control was done using a DNA High Sensitivity Kit on a Agilent 2100 Biooanalyzer (Agilent Technologies; Santa Clara, USA). Amplification of DNA was repeated with the same Index primers for low concentrated samples (¡1.5nmol) and clean-up of DNA was repeated in case of residual adapters and/or primers. 
Table 3.6 - Protocol for qPCR of ChIP samples using NEBNext Q5 polymerase

\begin{tabular}{|l|c|}
\hline Component & Volume \\
\hline $\begin{array}{l}\text { NEBNext Q5 Hot Start HiFi PCR } \\
\text { Master Mix }\end{array}$ & $2.5 \mu \mathrm{l}$ \\
\hline NEBNext Index Primer & $0.1 \mu \mathrm{l}$ \\
\hline NEBNext Universal Primer & $0.1 \mu \mathrm{l}$ \\
\hline $\begin{array}{l}\text { SyBr Green (04887352001 from } \\
\text { Roche; Basel, Switzerland) }\end{array}$ & $0.3 \mu \mathrm{l}$ \\
\hline DNA sample & $2.00 \mu \mathrm{l}$ \\
\hline
\end{tabular}

\begin{tabular}{|l|c|c|}
\hline Step & Temperature & Duration \\
\hline pre-incubation & $95^{\circ} \mathrm{C}$ & $30 \mathrm{~s}$ \\
\hline \multirow{2}{*}{$\begin{array}{l}\text { amplification } \\
45 \mathrm{x}\end{array}$} & $98^{\circ} \mathrm{C}$ & $10 \mathrm{~s}$ \\
\cline { 2 - 3 } & $65^{\circ} \mathrm{C}$ & $75 \mathrm{~s}$ \\
\hline cooling & $40^{\circ} \mathrm{C}$ & $10 \mathrm{~s}$ \\
\hline
\end{tabular}

Left: qPCR reaction solution. Right: Light cycler program.

\subsubsection{Methylated DNA immunoprecipitation (MeDIP)}

Methylated DNA immunoprecipitation (MeDIP) is a technique to unravel potential changes in DNA methylation and was performed as described in (Halder et al., 2015). Sheared chromatin can serve as starting material for MeDIP, however, one first has to isolate the DNA as described above (see section 3.2.8, p.39ff.). 200ng of sheared chromatin were used for each sample and DNA pellets were resuspended in $55 \mu$ l elution buffer. Then, DNA fragments underwent end repair and adapter ligation as described in section 3.2.10 (see p.40) using the NEBNext Ultra DNA Library Prep Kit (NewEngland Biolabs; Ipswich, USA). 41.25 $\mu$ l AMPure XP beads (Beckman Coulter; Breas, USA) were added to each sample to clean the ligated DNA from adapters and to perform size selection. After vortexing, the samples were incubated for $7 \mathrm{~min}$ at RT and placed on a magnetic rack afterwards for 5 minutes. The clear supernatant was removed without disturbing the magnetic beads, bound to the DNA. Beads were washed and incubated with $200 \mu \mathrm{l}$ $80 \%$ ethanol for 30s at RT twice. The ethanol was carefully removed, and the beads were dried for $10 \mathrm{~min}$ before eluting the DNA into $22 \mu \mathrm{l}$ elution buffer. The clear supernatant was separated from the beads and again mixed with $20 \mu \mathrm{l}$ AMPure XP beads. DNA was isolated once again. After a final resuspension of beads in $27 \mu$ l elution buffer, samples were incubated for 2 minutes at RT and the DNA containing clear buffer was extracted from the beads.

$0.1 \mu$ g of size selected DNA were mixed with $2 \mu \mathrm{l}$ blocking DNA and $38 \mu \mathrm{l}$ TE buffer. These blocking DNA resembles 20bp sequences complementary to the used adaptors. Samples were denatured at $99^{\circ} \mathrm{C}$ for $10 \mathrm{~min}$ and immediately placed on ice for another $10 \mathrm{~min}$. The DNA was mixed with $5 \mu \mathrm{l}$ 
of 10x IP buffer, $1 \mu \mathrm{l}$ of 1:5 diluted 5mc antibody (BI-MECY-0500 from Eurogentec; Liège, Belgium) and $2 \mu \mathrm{l}$ Dynabeads Protein G (Life technologies; Carlsbad, USA) and incubated for $6 \mathrm{hr}$ at $4^{\circ} \mathrm{C}$ on a rotation wheel. Afterwards, the beads were isolated and washed thrice with $700 \mu \mathrm{IP}$ buffer by incubating the tubes for $10 \mathrm{~min}$ at $4^{\circ} \mathrm{C}$ on a rotation wheel. The beads were isolated again, mixed with $30 \mu$ l Proteinase K digestion buffer containing $70 \mu$ g proteinase K. DNA was isolated as described in section 3.2.8 (see p.39).

The number of cycles needed for amplification of DNA were estimated by qPCR using the TruSeq PCR Primer Cocktail (Illumina; San Diego, USA) and Phusion HF PCR Master Mix (NewEngland Biolabs; Ipswich, USA) according to table 3.7. Samples were amplified for ct-2 cycles using $9 \mu \mathrm{l}$ DNA mixed with $0.5 \mu \mathrm{l}$ TruSeq PCR Primer cocktail and 9.5 $\mu$ l Phusion HF PCR Master Mix and the same PCR protocol as used for qPCR. Afterwards, the DNA was isolated as described above (see section 3.2 .10$, p.40).

Table 3.7 - Protocol for qPCR of MeDIP samples using Phusion HF polymerase

\begin{tabular}{|l|c|}
\hline Component & Volume \\
\hline PCR-graded $\mathrm{H}_{2} \mathrm{O}$ & $3.4 \mu \mathrm{l}$ \\
\hline $\begin{array}{l}\text { TruSeq PCR Primer Cocktail } \\
(25 \mu \mathrm{M})\end{array}$ & $0.4 \mu \mathrm{l}$ \\
\hline Phusion HF PCR Master Mix (2x) & $5.0 \mu \mathrm{l}$ \\
\hline $\begin{array}{l}\text { SyBr Green (04887352001 from } \\
\text { Roche; Basel, Switzerland) }\end{array}$ & $0.2 \mu \mathrm{l}$ \\
\hline DNA sample & $1 \mu \mathrm{l}$ \\
\hline
\end{tabular}

\begin{tabular}{|l|c|c|}
\hline Step & Temperature & Duration \\
\hline pre-incubation & $98^{\circ} \mathrm{C}$ & $45 \mathrm{~s}$ \\
\hline \multirow{3}{*}{$\begin{array}{l}\text { amplification } \\
30 \mathrm{x}\end{array}$} & $98^{\circ} \mathrm{C}$ & $15 \mathrm{~s}$ \\
\cline { 2 - 3 } & $65^{\circ} \mathrm{C}$ & $30 \mathrm{~s}$ \\
\cline { 2 - 3 } & $72^{\circ} \mathrm{C}$ & $30 \mathrm{~s}$ \\
\hline denaturation & $72^{\circ} \mathrm{C}$ & $60 \mathrm{~s}$ \\
\hline cooling & $4^{\circ} \mathrm{C}$ & $\infty$ \\
\hline
\end{tabular}

Left: qPCR reaction solution. Right: Light cycler program. 


\subsection{NGS data analysis}

Raw data from Next-Generation Sequencing were quality controlled using FastQC (Andrews, 2010). Handling of raw data including generation of BAM files and RNAseq count files as well as differential expression analysis were done in collaboration with the lab of Stefan Bonn (German Center for Neurodegenerative Diseases; Göttingen, Germany).

\subsubsection{Differential gene expression}

Alignment of RNAseq data was done using the Mus musculus mm10 reference genome and STAR aligner (Dobin et al., 2013) for generating BAM files. RNA count files were created with FeaturesCount (Liao et al., 2013) and differential expression analysis was done using DESeq2 (Love et al., 2014).

Genes with an adjusted p-value $\leq 0.05$ were considered as significantly differentially expressed. Additionally, a cutoff for $\log _{2}$ fold-changes of \pm 0.5 was applied on gene expression data unless otherwise stated and only those transcripts that code for actual proteins were considered.

Distance heatmaps showing the euclidean distance between samples and plots for principle compnent analysis were generated using the R packages DESeq, DESeq2, gplots and RColorBrewer (Anders and Huber, 2010; Love et al., 2014; Neuwirth, 2014; R Core Team, 2015; Warnes et al., 2015) to visualize clustering of samples (see code 3.1, p.51). Heatmaps for fold changes of significantly differentially expressed genes were generated using the R packages Biobase and DESeq2 (Huber et al., 2015; Love et al., 2014) along with the previously used packges gplots and RColorBrewer (see code 3.2, p.52).

To visualize a longitudinal pattern of differential gene expression, all genes which were differentially expressed in at least one of the respective comparisons were clustered using the Partition Around Medoids technique and plotted in a heatmap using R (see code 3.4, p.52) (Maechler et al., 2015). The number of clusters was set to 7 to enable clustering of all possible intersects of genes. Functional pathway analysis of differential gene expression data was done with the commercial, 
Java based tool Ingenuity Pathway Analysis (http://www.ingenuity.com, Qiagen; Venlo, Netherlands) and DAVID Bioinformatics Resources (https://david.ncifcrf.gov) regarding KEGG (Kyoto Enzyclopedia of Genes and Genomes) pathways. Only the top 10 "Ingenuity pathways" of one comparison were plotted for visual clarity purposes. P-values were computed using fisher's exact t-test. Heatmaps for functional pathways were generated using the R-packages gplots and RColorBrewer (Neuwirth, 2014; R Core Team, 2015; Warnes et al., 2015).

\subsubsection{Transcription factor binding site prediction}

To identify potential core regulators of differential gene expression, Pscan (vers.1.3) was used to screen for overrepresented TFs (Zambelli et al., 2009). All differentially expressed genes (see section 3.3.1, p.44) of a comparison were taken as input and Pscan was run with the following preferences: Mus musculus (organism), -200bp to +50bp (region around TSS), Jaspar 2014 (descriptor). EnsembliDs from the differential expression tables were converted to RefSeqIDs using BioMart (http://www.ensembl.org/biomart/) and R (R Core Team, 2015).

Adjusted p-values for the whole set of TFs were computed with R (see code 3.3, p.52). Only those TFs with an adjusted p-value of 0.05 or less and a motif length of at least $8 \mathrm{bp}$ were used for further analysis. Differentially expressed genes with a score of 0.95 or higher for a respective TF were considered as likely target genes. The top 20 motifs were plotted for visual clarity.

\subsubsection{Differential splicing}

Raw count files from RNAseq were analyzed for differential exon usage using the R-package "DEXSeq" (see code 3.6, p.53) (Anders et al., 2012; R Core Team, 2015). Exons with an adjusted p-value of 0.1 and a log2foldchange of \pm 0.15 were considered as significantly differentially expressed. Functional (KEGG-)pathway analysis of genes containing significantly differentially expressed exons was done using DAVID Bioinformatics Resources (https://david.ncifcrf.gov) (Huang et al., 2008, 2009). Correlation analysis between gene expression and alternative splicing was done using R (see code 3.5, p.52). 


\subsubsection{MeDIP- and ChIPseq data analysis}

Differential DNA methylation was analyzed using the R package MEDIPS (Lienhard et al., 2014) as described in source code 3.7 (see p.54). MACS was used for calling significant peaks of ChIPsequencing (Zhang et al., 2008) and differential binding was analyzed using the R package DiffBind with default parameters (Stark and Brown, 2011). ChIPSeeker was used for annotating identified peaks to the reference genome Mus Musculus mm10 (Carlson and Maintainer, 2015; Yu et al., 2015).

NGS plots showing the average DNA- and H3K4-methylation along gene bodies of low, mid and high expressed genes and the NGS pie charts showing the relative distribution of peaks within the genome were generated with the standalone software ngs.plot (Shen et al., 2014). Low and High expressed genes resemble the bottom and top 0.1 quantiles of normalized read counts respectively. Mid expressed genes resemble quantile 0.25 to 0.35 . Functional pathway analysis was done using DAVID Bioinformatics Resources (https://david.ncifcrf.gov) (Huang et al., 2008, 2009). Correlation studies were done with $\mathrm{R}$ as described (see code 3.5, p.52).

Identification of long distance interactions was done based on published Hi-C data from the mouse cortex (Dixon et al., 2012). Hot-spots of gene expression were defined as long distance interaction domains in which at least $20 \%$ of the included genes are differentially expressed.

\subsubsection{Drug screening}

The Java based and freely available Drug Pair Seeker (Zhong et al., 2013) was used for a direct approach to uncover potential therapeutic drugs based on the set of differentially expressed genes. The Drug Pair Seeker compares an input set of deregulated genes with published data from the CMAP database (Lamb et al., 2006) where approximately 1300 drugs were tested on human cancer cell-lines. The top 10 pairs of drugs for each comparison (wildtype vs transgenic; aging in transgenic mice) and brain region were collected and individually evaluated for their bioavaiablity, mechanism of action, potential side effects and published data considering the drugs. 


\subsection{Behavioral experiments}

\subsubsection{Drug administration}

Four months old male and female APP/PS1 transgenic mice were treated with either vehicle $\left(\mathrm{n}_{0^{7}}=5, \mathrm{n}_{\odot}=5\right), 125 \mathrm{mg} / \mathrm{l}$ SAHA $\left(\mathrm{n}_{0^{7}}=6, \mathrm{n}_{\varphi}=5\right), 50 \mathrm{mg} / \mathrm{l}$ memantine $\left(\mathrm{n}_{\mathrm{O}^{7}}=7, \mathrm{n}_{\varphi}=3\right)$ (both from Cayman Chemicals; Ann Arbor, USA) or a combination of both medications $\left(\mathrm{n}_{\mathrm{O}^{\top}}=8, \mathrm{n}_{\varphi}=5\right)$ in potable water containing 18g/l (2-Hydroxypropyl)- $\beta$-cyclodextrin (Sigma Aldrich; St. Louis, USA). To find a potential beneficial effect of the two drugs when given in combination, dosages were arbitrary chosen where the individual drugs were not suspected to cause their full therapeutic potential previously described (Barnes et al., 1996; Benito et al., 2015; Guan et al., 2009; Minkeviciene et al., 2004). Assuming an average body weight of $30 \mathrm{~g}$ and drinking volume of $3 \mathrm{ml}$ per day, the given concentrations resemble $12.5 \mathrm{mg} / \mathrm{kg}$ SAHA and $5 \mathrm{mg} / \mathrm{kg}$ memantine per day respectively.

The cyclodextrin was added to increase solubility and bioavailability as well as to cover any potential flavor caused by the respective therapeutic drugs (Jambhekar and Breen, 2015). Treatment of mice started four weeks before animal experiments and lasted until the last day of behavioral experiments.

\subsubsection{Open field}

Mice were tested in the open field test to study explorative behavior and locomotion ( $\mathrm{n}=9$ for vehicle, memantine or SAHA, $n=13$ for combinatory treatment). A mouse was placed into the center of an open quadratic arena (50x50 cm, 20cm high walls) and recorded for 5 minutes using the VideoMot2 system (TSE Systems; Bad Homburg, Germany). The overall traveled distance and the proportion of time the mice spent within the arena's center (more than $10 \mathrm{~cm}$ distance to the walls) are conventionally quantified for indicating locomotive and explorative behavior respectively (Carola et al., 2002). 


\subsubsection{Elevated plus maze}

For analyzing basal anxiety levels, mice were tested in the elevated plus maze ( $n=9$ for vehicle, memantine or SAHA, n=13 for combinatory treatment). The maze consisted of two open and two enclosed arms ( $1 \mathrm{x}$ w: $30 \times 9 \mathrm{~cm}, 20 \mathrm{~cm}$ high walls) without ceiling which were connected by a $9 \mathrm{~cm}^{2}$ large center area. It was placed on a metal stand at a height of $75 \mathrm{~cm}$. A mouse was placed into the center of the maze and recorded for 5 minutes using the VideoMot2 tracking system. The relative time a mouse spent in open and enclosed arms was quantified, whereby a pronounced preference for enclosed arms indicated a higher basal anxiety (Carola et al., 2002).

\subsubsection{Morris water maze}

The morris water maze is a behavioral experiment to investigate spatial memory in mice (Morris, 1981). A mouse was placed into a round basin (1.2m in diameter) filled with opaque water, unable to escape the basin by itself. A quadratic platform $(10 \times 10 \mathrm{~cm})$ was placed right underneath the water surface, thus hidden from the mice's view. The basin was sectioned in 4 equal quadrants, each with an optic cue attached to the basin's wall. The platform was placed in the center of one random section and remained in the same position for the entire experiment.

During the training days, a mouse was placed in each of the four quadrants once and was allowed to explore the basin until finding the platform and remaining on it for 10 seconds. Mice that did not find the platform within 60s were placed onto the platform afterwards to ensure memory formation. Training was done for 10 consecutive days. One the sixth and eleventh day, a probe test was performed. The platform was removed prior to the test and each mouse was allowed to explore the basin for 1 minute starting at the quadrant opposite to the initial platform's position ( $\mathrm{n}=9$ for vehicle, memantine or SAHA, $\mathrm{n}=13$ for combinatory treatment). Three days after the second probe test, mice underwent a reversal learning test to investigate memory plasticity ( $\mathrm{n}_{\text {vehicle }}=5, \mathrm{n}_{S A H A}=5, \mathrm{n}_{\text {Memantine }}=9, \mathrm{n}_{\text {combi }}=9$ ) (Vorhees and Williams, 2006). Therefore, the platform was placed in the opposite quadrant of its initial position and mice were trained for another three days as previously described. A final probe test was done at the fourth day of reversal learning. 
The latency to discover the platform during each learning trial was quantified to assess information on spatial memory formation. The percentage of time spent in the target quadrant and the number of visits to the initial platform's position during the probe test were measured as indicators for spatial memory retrieval.

\subsubsection{Contextual fear conditioning}

To analyze contextual fear memory, mice underwent Pavlovian contextual fear conditioning using an automated fear conditioning setup for mice (Med Associates; St. Albans, USA). Similar to previous studies, a mouse was placed into a conditioning chamber $(30.5 \times 24.1 \times 21.0 \mathrm{~cm}, 1$ $\mathrm{x} \mathrm{w} \mathrm{x} \mathrm{h)} \mathrm{with} \mathrm{controlled} \mathrm{visual} \mathrm{and} \mathrm{auditory} \mathrm{stimuli} \mathrm{(Agis-Balboa} \mathrm{et} \mathrm{al.,} \mathrm{2011).} \mathrm{The} \mathrm{floor} \mathrm{of} \mathrm{the}$ chamber was a metal grid used to transmit electric shocks to the test subjects.

For training, mice were allowed to explore the chamber for 3 minutes until they received a footshock ( $0.7 \mathrm{~mA})$ for 2 seconds. 24 hours later, they were reintroduced to the very same context and their locomotive behavior was assessed. The relative amount of freezing behavior indicated contextual fear memory, whereby freezing in mice describes the absent of significant motion. 


\subsection{Statistics and graphical output}

Simple calculations and table handling was done with Microsoft Excel 2010 (Microsoft; Redmond, USA). Statistical analyses, data mining and batch processing of data was done in R (R Core Team, 2015). Correlation analyses and linear regressions were generated as described in code 3.5 (see p.52). A list of all used R packages is displayed in table 3.8 (see p.51). Statistical evaluation of behavioral experiments was done in Prism 6 (GraphPad Software; La Jolla, USA). Significance for whole data sets were tested with one-way and two-way ANOVA. Tukey's multiple comparison test was used for one-to-one comparisons within groups. A potential significant difference from chance level in the Morris Water Maze was tested via two-tailed t-test. Potential effects of gender or batch affiliation on mice behavior was tested by two-way ANOVA. Behavioral differences with a p-value $\leq 0.05$ were considered as significant and were highlighted in plots by an asterisk.

Adobe Illustrator CS5.1 (Adobe Systems Inc.; San José, USA) was used to generate Venn diagrams and pie charts, and to reformat graphical outputs from GraphPad Prism and R. In full compliance with the general terms on good scientific practice, adjustments on data plots were exclusively made on fonts, colors, and global scaling without affecting the actual data. 


\subsection{R source codes}

Table 3.8 - List of R packages

\begin{tabular}{|c|l|}
\hline Package & citation \\
\hline Biobase & Huber et al. (2015) \\
\hline BSgenome & Pages (2016) \\
\hline BSgenome.Mmusculus.UCSC.mm10 & The-Bioconductor-Dev-Team \\
\hline ChIPseeker & Yu et al. (2015) \\
\hline cluster & Maechler et al. (2015) \\
\hline DESeq & Anders and Huber (2010) \\
\hline DESeq2 & Love et al. (2014) \\
\hline DEXSeq & Anders et al. (2012) \\
\hline DiffBind & Stark and Brown (2011) \\
\hline GenomicFeatures & Lawrence et al. (2013a) \\
\hline GenomicRanges & Lawrence et al. (2013b) \\
\hline gplots & Warnes et al. (2015) \\
\hline MEDIPS & Lienhard et al. (2014) \\
\hline openxlsx & Walker (2015) \\
\hline org.Mm.eg.db & Carlson (2015) \\
\hline RColorBrewer & Neuwirth (2014) \\
\hline FeaturesCount & Liao et al. (2013) \\
\hline TxDb.Mmusculus.UCSC.mm10.ensGene & Carlson and Maintainer (2015) \\
\hline &
\end{tabular}

Code 3.1 - Generating distance heatmaps in $\mathrm{R}$

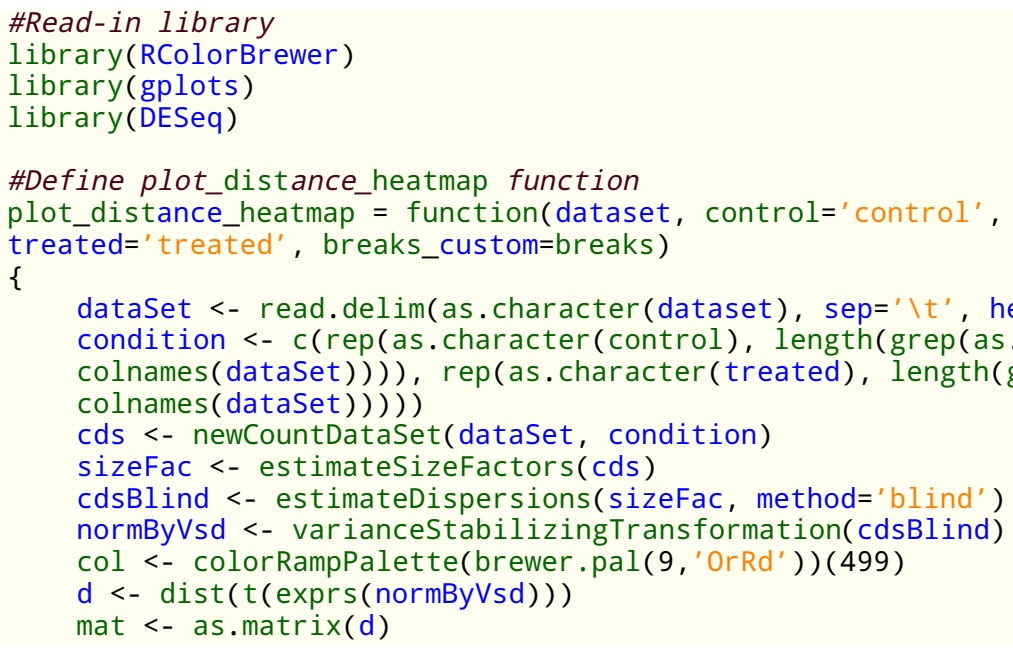




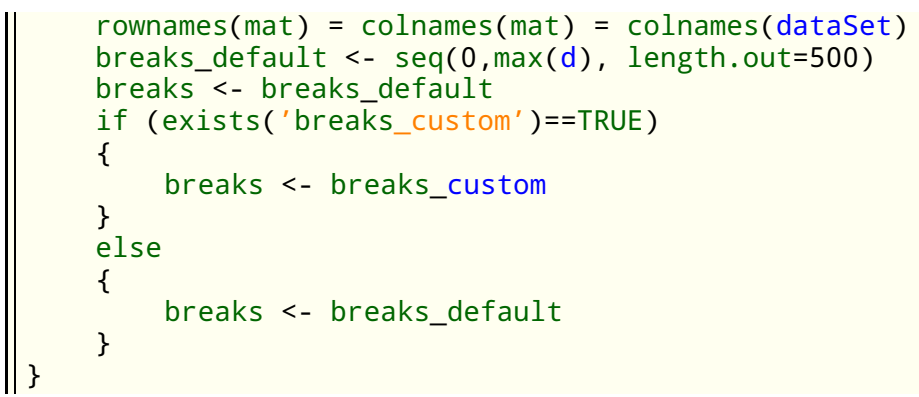

Code 3.2 - Generating heatmaps in $\mathrm{R}$

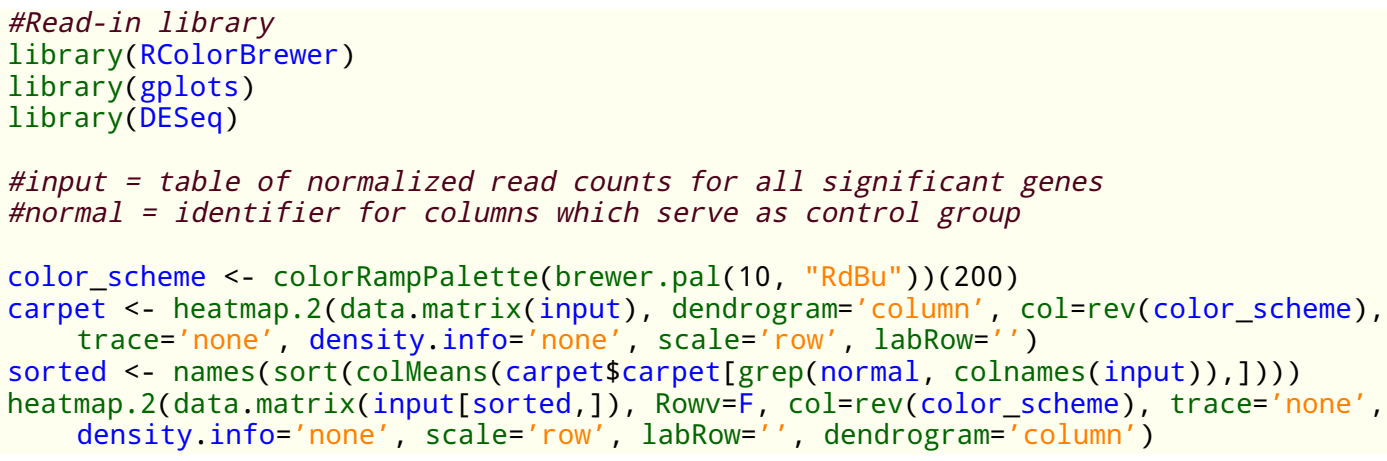

Code 3.3 - Computing adjusted p-values in $\mathrm{R}$

|| padj <- p.adjust(pvalue, "fdr", length(pvalue))

Code 3.4 - Partitioning Around Medoid clustering in $\mathrm{R}$

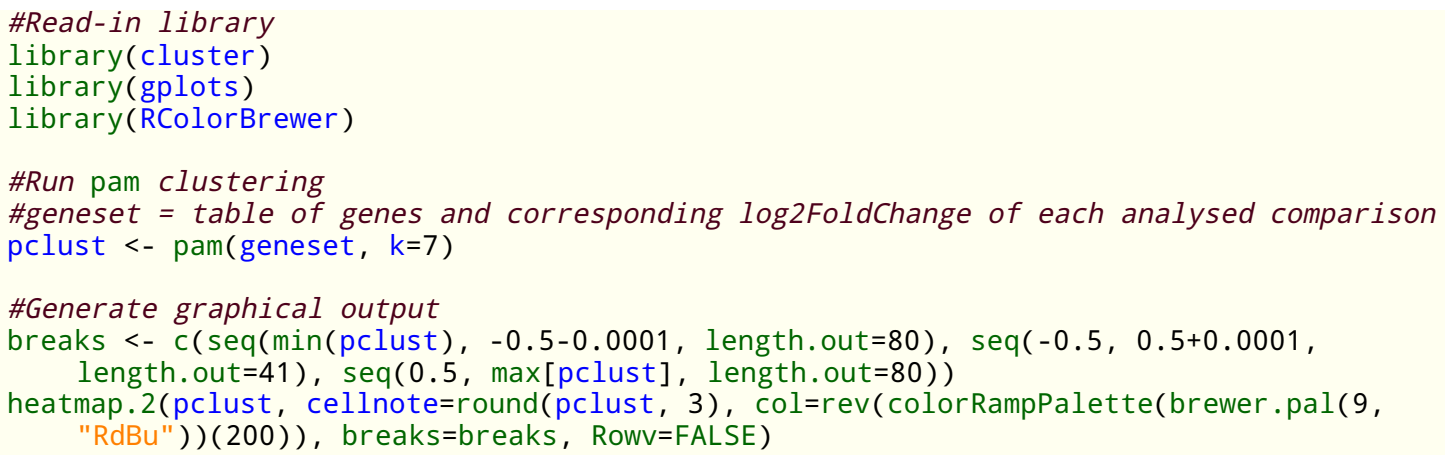

Code 3.5 - Correlation and linear regression in $\mathrm{R}$

\#input = dataframe of values of choice from a single comparison (i.e. wildtype vs transgenic mice, or gene expression vs splicing).

plot (input\$A, input\$B)

abline ( $\operatorname{lm}\left(\right.$ input $\$ A^{\sim}$ input $\left.\$ B\right)$ )

r_square <- cor (input $\$ A$, input $\$ B)^{\wedge} 2$ 
Code 3.6 - Differential Splicing analysis using DEXSeq in $\mathrm{R}$

\#Read-in library

library(DEXSeq)

\#countFiles = list of countfiles to be analysed

\#sampleTable = list of sample names and corresponding condition (i.e. 4mo transgenic ACC)

\#flattenedFile $=$. gff file

\#set number of CPU cores to work on

BPPARAM = MulticoreParam (workers $=$ cores $)$

\#execute DEXSeq

$\mathrm{d} x \mathrm{~d}$ = DEXSeqDataSetFromHTSeq(countFiles, sampleData=sampleTable, design= sample + exon + condition:exon, flattenedfile=flattenedFile)

$\mathrm{d} x \mathrm{~d}=$ estimateSizeFactor $\mathrm{s}(\mathrm{d} \mathbf{x d})$

$d x d=$ estimateDispersions $(d x d$, BPPARAM=BPPARAM $)$

$\mathrm{d} x \mathrm{~d}=$ testForDEU $(\mathrm{dxd}$, BPPARAM=BPPARAM $)$

$d x d=$ estimateExonFoldChanges $(d x d$, fitExpToVar="condition", BPPARAM=BPPARAM $)$

$\mathrm{d} \times \mathrm{r} 1=$ DEXSeqResults $(\mathrm{d} x \mathrm{~d})$

\#create output files

DEXSeqHTML (dxr1, file, FDR=0.1, color=c("\#FF000080", "\#0000FF80"), BPPARAM=BPPARAM )

write.csv(as.data.frame(dxr1), file) 
Code 3.7 - MeDIP-sequencing analysis using MEDIPS in $\mathrm{R}$

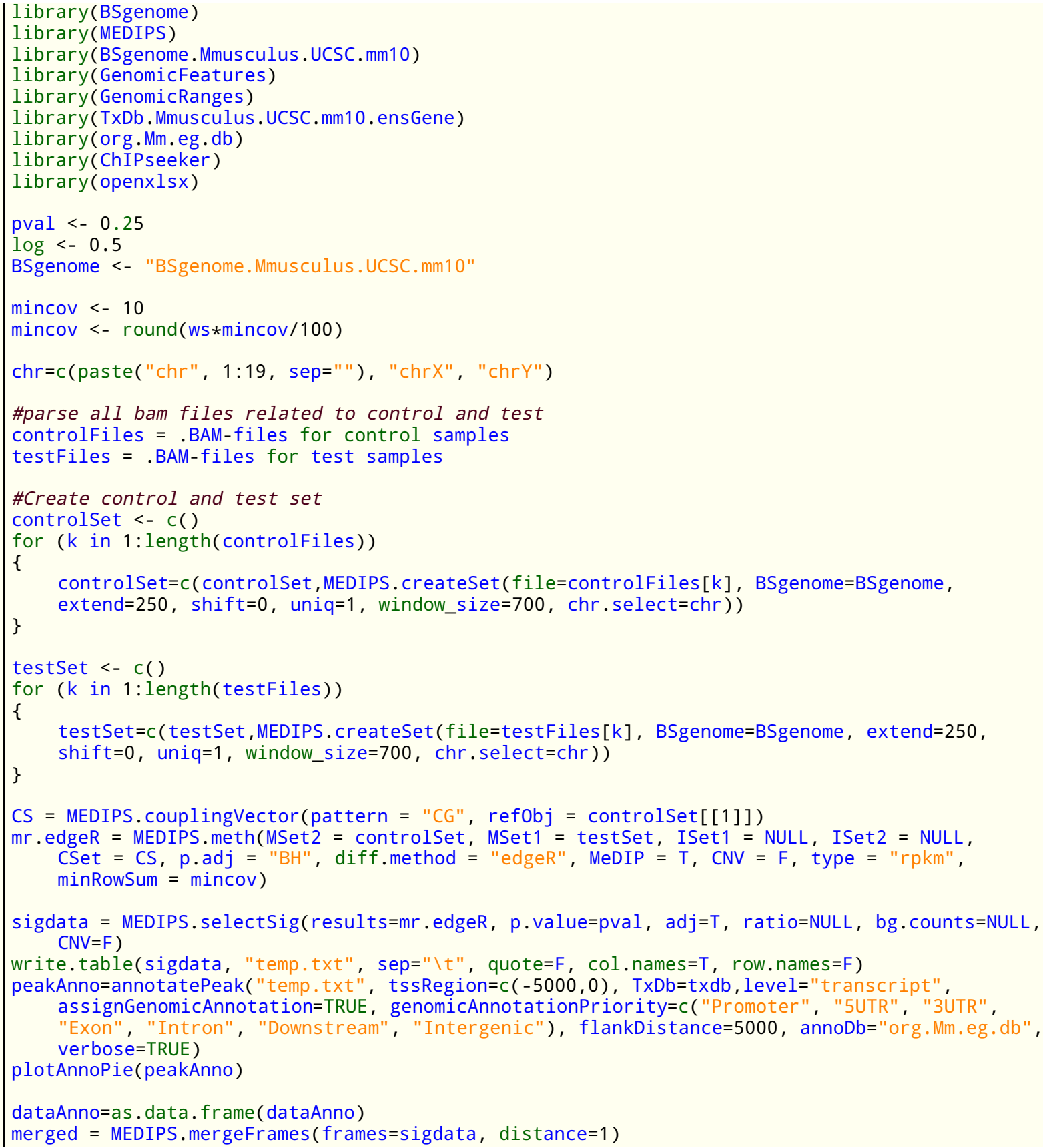




\subsection{Buffers and solutions}

\begin{tabular}{ll} 
Elution buffer (pH 8) & 10mM Tris \\
\hline High sucrose buffer (ph 8) & $\begin{array}{l}1 \mathrm{M} \text { Sucrose, 3mM MgAc } 2,50 \mathrm{mM} \mathrm{HEPES,} \mathrm{1mM} \\
\text { DTT }\end{array}$ \\
\hline IP buffer (pH 8) & $\begin{array}{l}\text { 150mM NaCl, 50mM Tris, 20mM EDTA, 1\% NP-40, } \\
0.5 \% \text { Sodium deoxycholate, 1\% SDS }\end{array}$ \\
\hline LiCl wash buffer (ph 8) & $\begin{array}{l}\text { 100mM Tris, 500mM LiCl, 20mM EDTA, 1\% NP-40, } \\
1 \% \text { Sodium deoxycholate }\end{array}$ \\
\hline Low sucrose buffer (pH 8) & $\begin{array}{l}\text { HEPES, 0.1mM EDTA, 1mM DTT, 0.1\% Triton-X } \\
\text { TE buffer (pH 8) }\end{array}$ \\
\hline PBTB & $\begin{array}{l}\text { 1x PBS, 0.1\% Tween, 50mg/ml BSA } \\
\text { RIPA buffer (pH 8) }\end{array}$ \\
\hline
\end{tabular}

Wienmann shearing buffer (pH 7.4) 10mM Tris, 20mM EDTA, 2\% SDS 


\section{Results}

\subsection{Transcriptional differences among brain regions}

For studying the molecular processes underlying the age-related cognitive decline in APP/PS1 mice, I chose three different areas of the murine brain involved in memory functioning and affected by amyloid depositions in AD patients. The ACC as a part of the frontal lobe is involved in consolidation and retrieval of LTM, the two hippocampal sub-regions CA1 and DG in encoding spatial and associative memory. To study longitudinal effects of the amyloid pathology on gene expression and epigenetic modifications, I selected three age groups resembling a healthy (1.5 months; young), early (4 months; mid-aged) and late-state (8 months; old) of the disease based on preliminary data regarding amyloidosis and mice behavior.

To highlight the importance of tissue-specific approaches, I analyzed interregional gene expression differences for the given brain areas. A useful statistical technique for clustering data is the Principal component analysis (PCA). In this technique, an orthogonal transformation of each variable of a dataset is made based on the actual values of this respective set of data (Jolliffe, 2002). Starting with a linear regression of the data that results in a maximal variance (PC1), an orthogonal vector is computed which by itself again shows the highest variance possible (PC2). By plotting principal component values (i.e. PC1 over PC2) for each sample, separate clusters can be identified. PCA was done for all age groups and revealed a clear separation of samples from the respective brain areas as it is exemplarily shown in figure 4.1A (see p.57) for young mice.

Figure 4.1B (see p.57) shows the relative euclidean distance among all samples where RNA was sequenced for. The euclidean distance as a measure for similarity or dissimilarity of measurements also enables clustering of samples. Three major clusters can be identified resembling the given brain regions. Clusters for the ACC and CA1 are relatively consistent, while samples from the DG seem to be more diverse. Two subclusters for the DG can be identified from the heatmap, whereby the samples inside these clusters cannot be separated by either age or genotype. Clusters resembling age groups or genotypes are in general overshadowed by the big dissimilarity of samples from the distinct brain regions. 
A
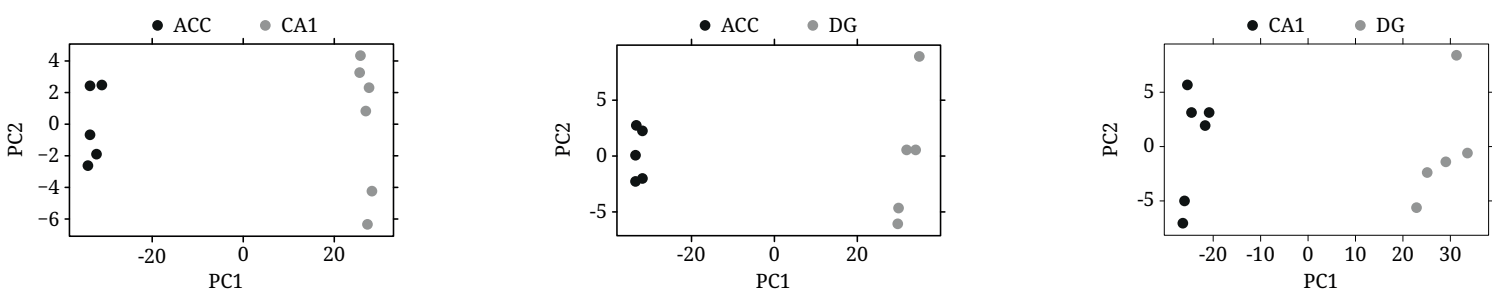

B

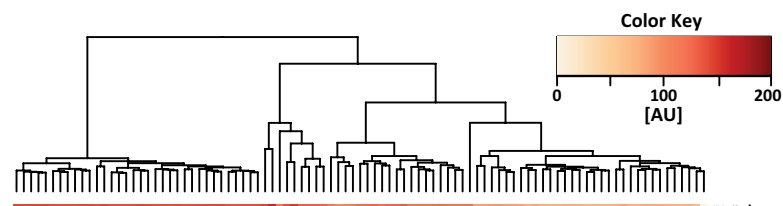

\section{C}

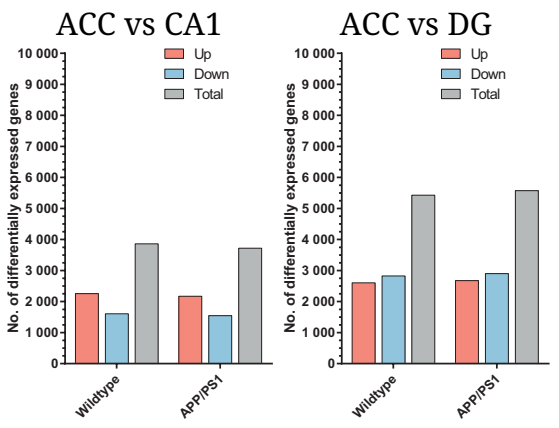

CA1 vs DG

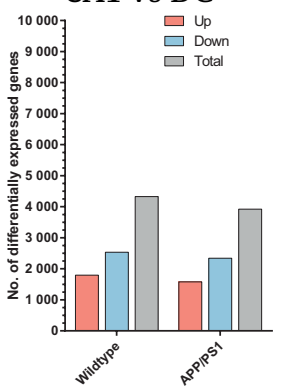

Figure 4.1 - Transcriptional differences among brain regions

A) PCA plots for interregional comparisons of ACC vs CA1 (left), ACC vs DG (center), and CA1 vs DG (right). Samples from different brain regions can clearly be separated by a straight line and thus belong to distinct clusters.

B) Euclidean distance between all sequenced RNA samples and clustering of these. The color key is ranging from $100 \%$ similarity (white) to dissimilarity (red) with increasing value (arbitrary unit). Three major clusters can be identified, namely samples from the different brain regions: ACC, CA1 and DG. No clusters can be observed resembling age groups or genotypes. A detailed listing of samples is shown in supplemental figure 7.1 (see p.129).

C) Number of differentially expressed genes between the given brain regions of 1.5 months old wildtype and transgenic mice. Up- and downregulation of genes is comparable in both genotypes.

The total number of significant genes differentially expressed among the brain regions ranges from 3861 (ACC vs CA1) to 5430 (ACC vs DG) in 1.5 months old wildtype mice (see figure 4.1C). Similar numbers of genes were observed in transgenic mice of the same age (ACC vs CA1: 3718; ACC vs DG: 5574). Within the hippocampal sub-regions CA1 and DG, 4328 genes were differentially expressed in young wildtype mice and 3920 in transgenic mice. Across all brain regions, protein coding RNA from 15,234 genes was detected by Illumina sequencing. Thus, the differentially expressed genes resemble approximately $25-35 \%$ of the whole transcriptome, emphasizing 
the difference between the tested brain areas. Functional pathways analysis was performed to discover cellular functions enriched by the set of differentially expressed genes. Identified pathways mainly resemble neuronal signaling, i.e. ”Axon guidance” or ”Neuroactive ligand-receptor interaction", and functions involved in cell survival and regulation of gene expression, i.e. "p53 signaling” or "Wnt signaling pathway" (see table 7.1, p.130).

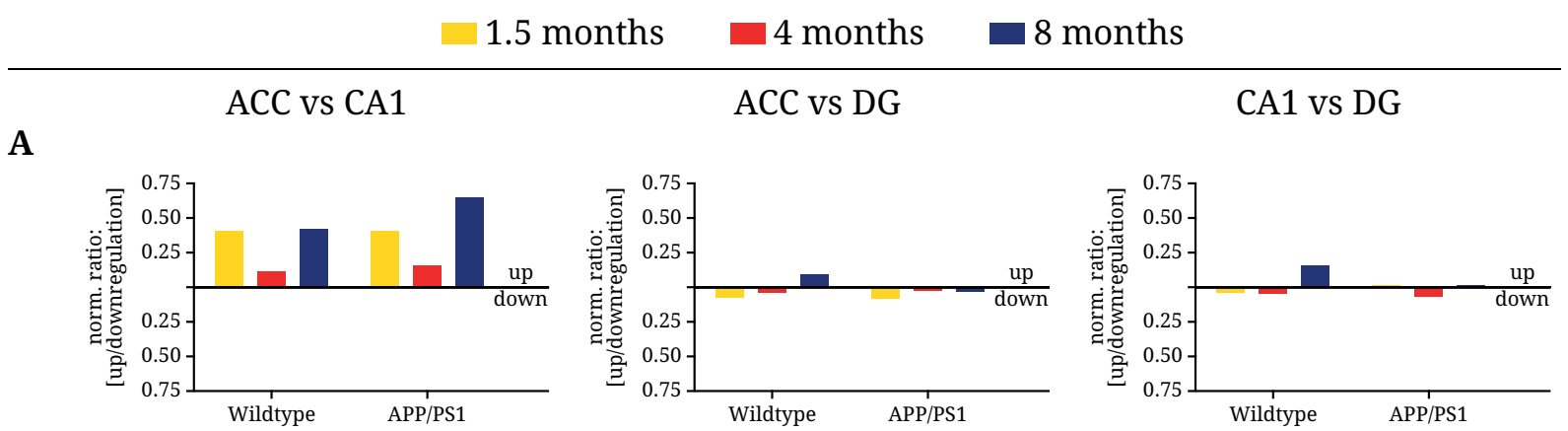

B
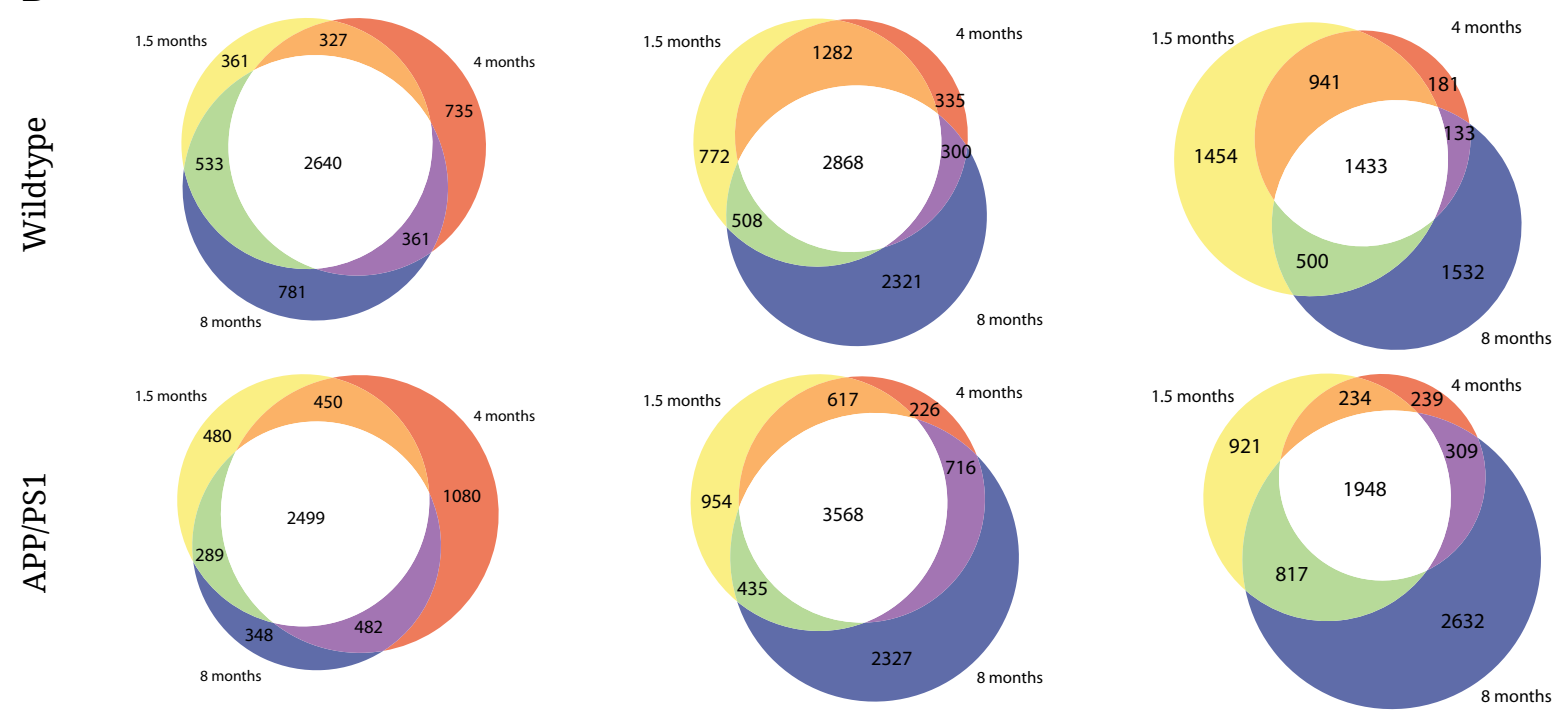

Figure 4.2 - Interregional differences: Percentage of upregulated genes:

A) Normalized ratio of up- and downregulated gene expression among brain regions for both genotypes and all given age groups. Values $>0$ resemble upregulation and vice versa.

B) Venn diagrams showing interregional differentially expressed genes common and uncommon between 1.5, 4, and 8 months old wildtype (top) and APP/PS1 transgenic (bottom) mice. The majority of genes is common between the age groups.

Transcriptional differences between brain regions remained relatively stable across all age groups and genotypes (see figure 4.2A). Compared to the ACC, genes are in average higher expressed in the CA1 in all age groups. Up- and downregulation of gene expression in the DG is 
rather evenly distributed compared to the ACC or CA1. Figure 4.2B (see p.58) shows the number of differentially expressed genes that are common or specific for the three age groups in each respective interregional comparison and genotype. When comparing the ACC to the CA1, the vast majority of genes is shared between all age groups. In contrast, comparisons of either the ACC or the CA1 with the DG revealed quite a high number of differentially expressed genes specific for a respective brain region in 8 months old mice. For instance, 2321 genes are exclusively differentially expressed when comparing the ACC to the DG in 8 months old wildtype mice compared to 3674 that are shared with other age groups in the same brain regions. Similar findings were made for APP/PS1 transgenic mice.

\subsection{Wildtype-like gene expression in young APP/PS1 mice}

Preliminary data from our lab showed, that no differences in cognitive performance can be observed in young APP/PS1 mice compared to wildtype littermates. Nevertheless, molecular processes might already be affected by the transgene driving the age related cognitive decline in those mice that can already be observed at an age of 4 months. I thus analyzed the gene expression profiles of the ACC, CA1, and DG from 1.5 months old APP/PS1 wildtype and transgenic mice and performed a cluster analysis as previously described (see section 4.1, p.56ff.). Distance heatmaps and PCA for all three brain regions of young wildtype and transgenic mice are shown in figure 4.3 (see p.60). The distance heatmaps clearly show that no distinct separation can be observed for either brain region. Samples from wildtype and transgenic mice can neither be separated by a single straight line in the PCA plots for either region further indicating their relative likeness. Samples can thus not be clustered on the basis of the whole transcriptome.

Despite there being no major differences between genotypes at 1.5 months of age, I was able to detect some amyloid-associated transcriptional changes. Figure 4.4 (see p.61) shows heatmaps only for the significantly differentially expressed genes in the given brain regions of young mice. Values represent relative foldchanges of deregulated genes in arbitrary units. Considering only the significant genes, samples from 1.5 months old wildtype and transgenic mice can nicely be separated as shown by the respective dendrograms. Forty-one genes are differentially expressed in the ACC of 1.5 months old mice, 28 of which are upregulated. In the CA1, 12 genes are up- 
A

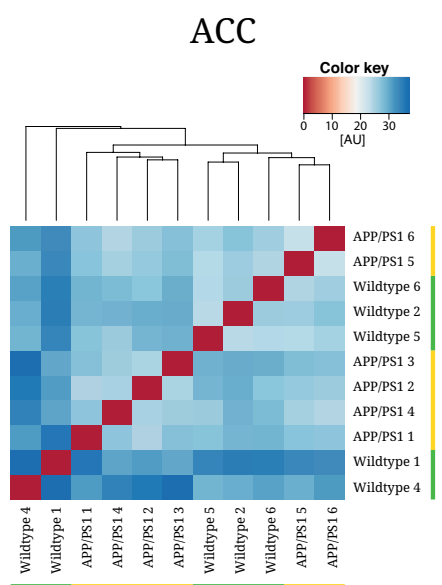

B
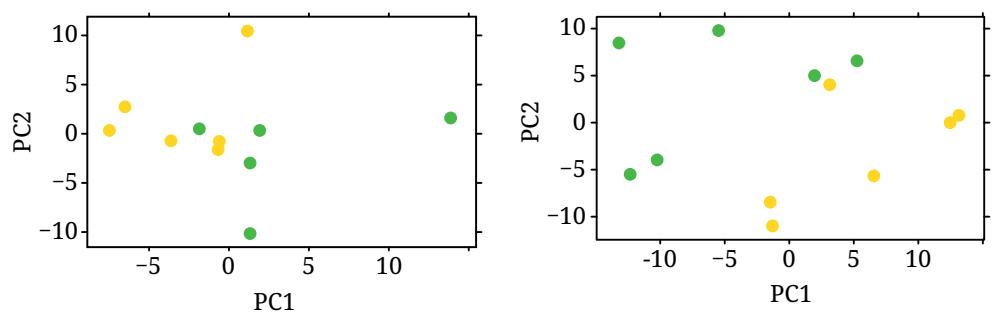

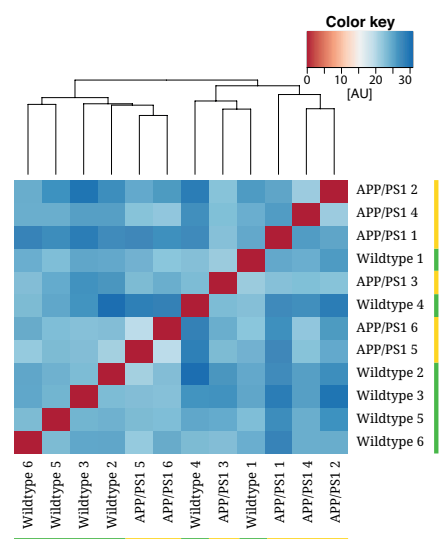

DG

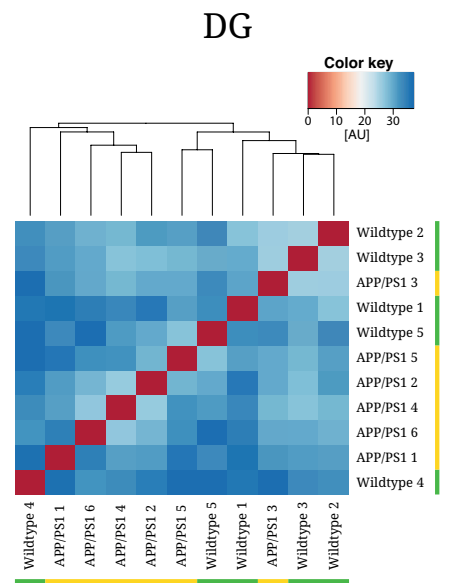

\section{- Wildtype}

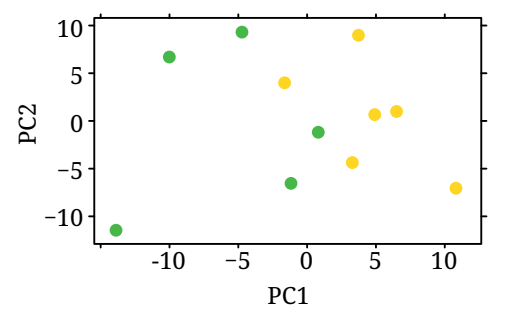

- APP/PS1

Figure 4.3 - Clustering of 1.5 months old mice:

A) Distance heatmaps showing euclidean distance of samples from all three brain regions of 1.5 month old mice. The color key is ranging from $100 \%$ similarity (red) to dissimilarity (blue) with increasing value (arbitrary unit). Dendrograms displayed above each heatmap show no distinct clustering of the two genotypes, highlighted by green (wildtype) and yellow (transgenic) bars respectively.

B) PCA plots for comparison of wildtype (green) and APP/PS1 transgenic (yellow) 1.5 months old mice. Samples from different genotypes cannot be separated by a straight line and thus cannot be clustered.

and 9 genes downregulated and in the DG, 17 genes are up- and 15 genes are downregulated. To discover potentially overrepresented pathways based on these genes, I screened for KEGG pathways enriched in the given datasets (see table 4.1, p.61). This analysis was done without a foldchange cutoff, increasing the number of input genes fore analysis to 348 in the ACC. The number of genes in the CA1 and DG remained relatively stable with or without a foldchange cutoff (CA1: 49, DG: 32 ).

Pathway analysis for the ACC revealed four significant pathways, namely "Endocytosis" $(p=0.0017)$, ”Notch signaling pathway" $(p=0.0091)$, ”Axon guidance” $(p=0.0209)$, and "Calcium signaling pathway" ( $\mathrm{p}=0.0382$ ). Only one pathway was significant for the CA1 ("Axon guidance", 
$\mathrm{p}=0.0043$ ) and none for the DG. Though only a slight tendency towards upregulation of gene expression can be observed in all three brain areas, the genes involved in the significant functional pathways are almost exclusively upregulated, with the exception of Vps37d, Lrrc4c, CD38, and Srgap1.

ACC

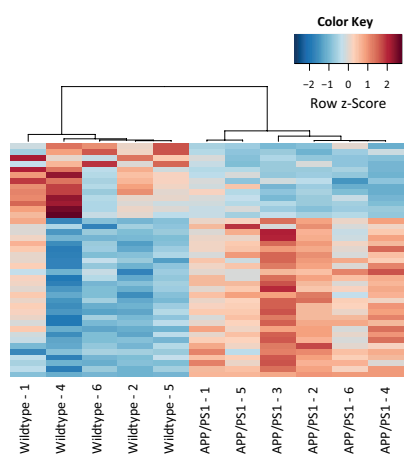

CA1

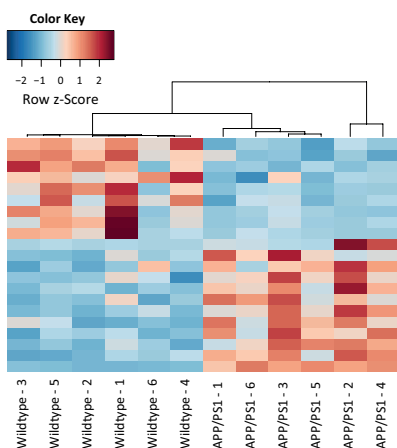

DG

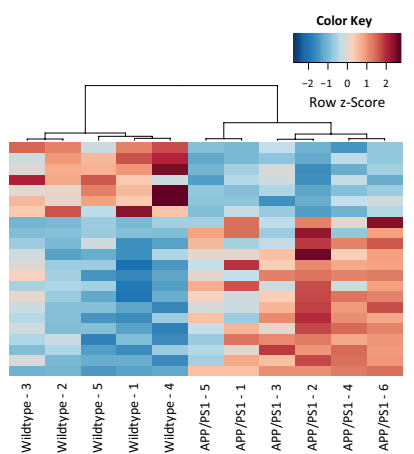

Figure 4.4 - Transcriptional differences in 1.5 months old mice:

Heatmaps for differentially expressed genes in the ACC, CA1, and DG of 1.5 months wildtype and APP/PS1 transgenic mice. Upregulation of gene expression is resembled by positive values and red boxes, downregulation by negative values and blue boxes.

Table 4.1 - Functional pathways in the ACC (top) and CA1 (bottom) of 1.5 months old mice. Downregulated genes are labeled in red.

\begin{tabular}{|c|c|c|c|}
\hline ACC & KEGG pathway & p-value & genes \\
\hline \multicolumn{2}{|c|}{ Endocytosis } & 0.0017 & $\begin{array}{l}\text { AGAP3, HGS, ACAP3, LDLR, EPN1, RAB11FIP3, PSD, } \\
\text { VPS37D, HSPA2, AP2A1, VPS37B }\end{array}$ \\
\hline \multicolumn{2}{|c|}{ Notch signaling pathway } & 0.0090 & JAG2, DVL1, NCOR2, DTX1, NUMBL \\
\hline \multicolumn{2}{|c|}{ Axon guidance } & 0.0209 & SEMA6B, SLIT1, UNC5A, LIMK1, EPHB6, EFNB1, LRRC4C \\
\hline \multicolumn{2}{|c|}{ Calcium signaling pathway } & 0.0382 & $\begin{array}{l}\text { GRIN2D, CACNA1H, GRIN1, HTR6, CD38, SLC8A2, GNA11, } \\
\text { PTK2B }\end{array}$ \\
\hline
\end{tabular}

\begin{tabular}{|l|l|c|l|}
\hline CA1 & KEGG pathway & p-value & genes \\
\hline Axon Guidance & 0.0043 & LRRC4C, SRGAP1, ABLIM3, MAPK3 \\
\hline
\end{tabular}




\subsection{Differential gene expression in APP/PS1 mice with mild and severe amyloidosis}

RNA from the ACC, CA1, and DG of APP/PS1 wildtype and transgenic mice was sequenced and compared to discover putative molecular mechanisms that might affect and partially drive the previously described cognitive decline in this mouse model for AD via gene expression profiles. Figure 4.5A (see p.63) shows the fold change and p-value of all protein coding transcripts identified by Illumina RNA sequencing in 1.5, 4, and 8 months old mice. A strong shift towards upregulation of gene expression can be observed in all three tested brain regions. This shift becomes more intense during aging and the number of significant genes rises with increasing age. The majority of genes with a p-value less than 0.05 is only mildly differentially expressed, meaning a $\log _{2}$ foldchange weaker than \pm 0.5 . As previously described, the gene expression profile of the DG is quite different from the ones observed for the ACC and CA1 (see section 4.1, p.56). Compared to the other brain regions, gene expression is only mildly affected in the DG of 4 months old mice and in general shows a broader and weaker distribution.

While samples from 1.5 months old mice were not yet separating significantly in the respective brain regions, wildtype and transgenic mice are becoming more diverse during aging. Compared to 41 genes that were differentially expressed in the ACC of young mice, 390 (363 up, 27 down) are already significantly affected in mid-aged and 839 (764 up, 75) in old mice (see figure 4.5B, p.63). In the CA1, 21 genes (12 up, 9 down) were differentially expressed at an age of 1.5 months, 326 (302 up, 24 down) at 4 months, and 856 (812 up, 44 down) at 8 months. In the DG of 8 months old mice, the number of significant genes rouse to 890 (542 up, 348 down) in older mice while the number of genes remained relatively stable in 4 months old mice compared to 1.5 months old mice (50 and 22 respectively).

To analyze the consistency of transcriptional changes during progressing age, I performed a partition around medoid (PAM) analysis. PAM is an advanced version of k-means clustering and can identify clusters of data-points that behave similar under different conditions - like age. PAM clustering was chosen as it is less susceptible to noise and outliers compared to its primitive technique of classical k-means clustering. 


\section{5 months $\quad 4$ months $\quad 8$ months}

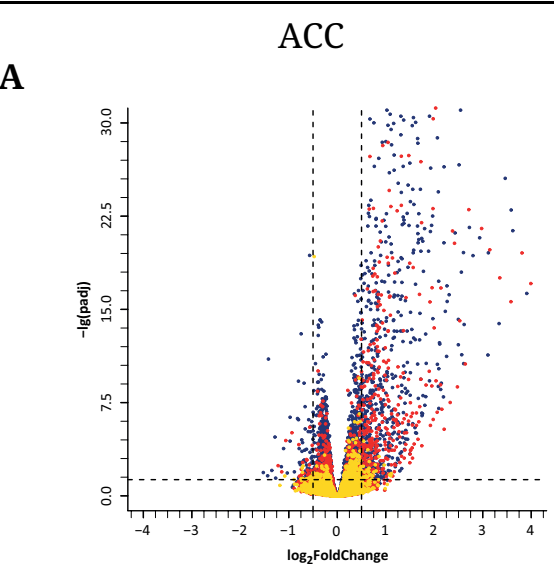

CA1

DG

B
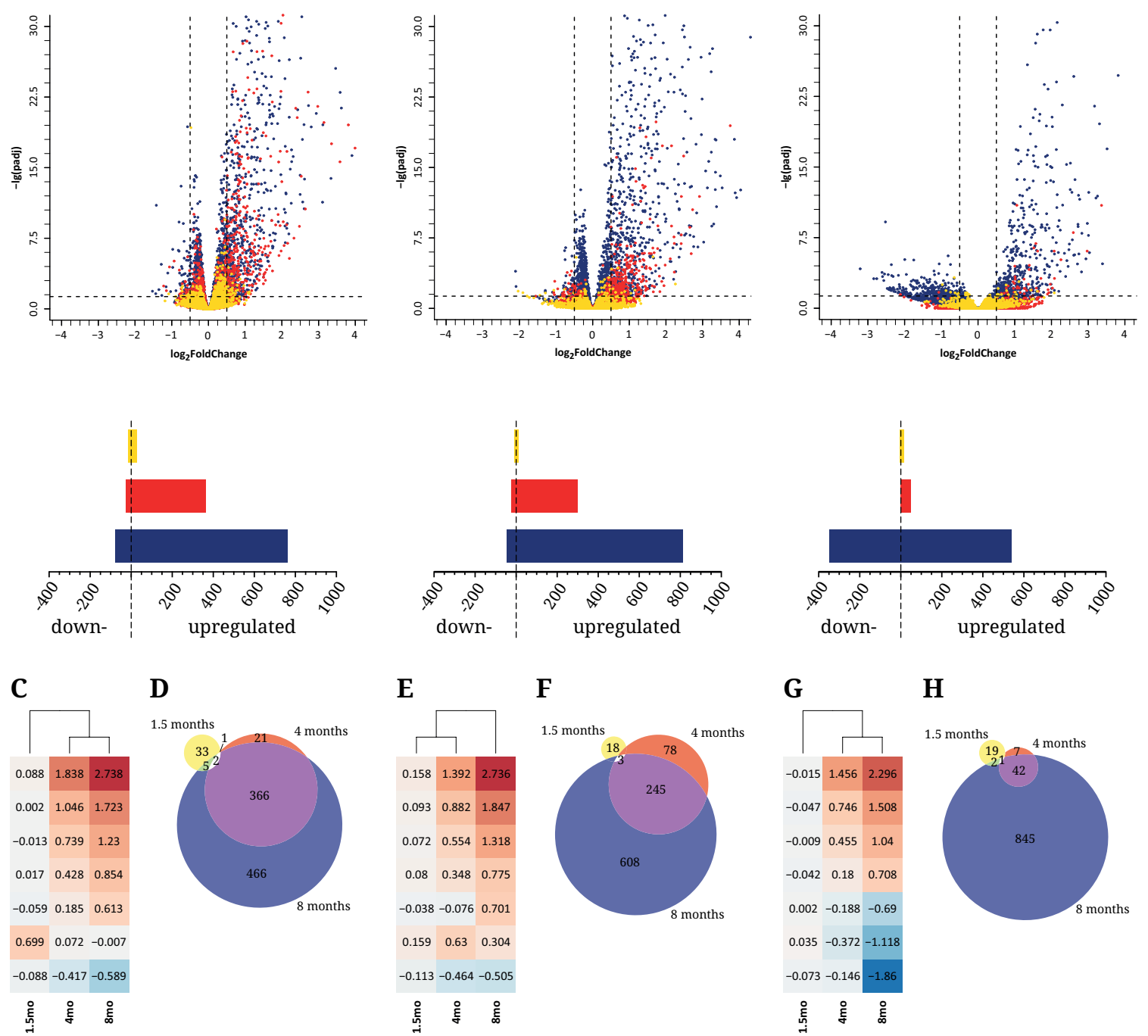

$\mathbf{E}$

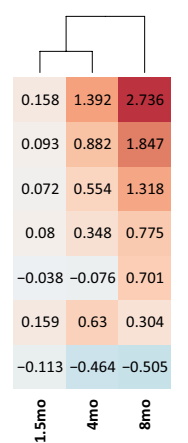

$\mathbf{F}$

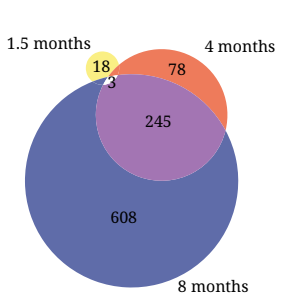

$\mathbf{H}$

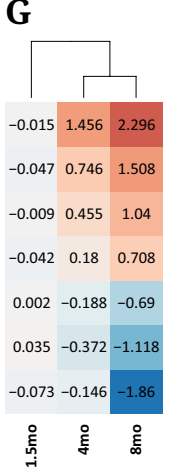

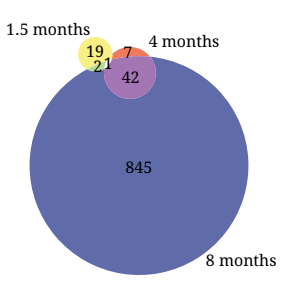

Figure 4.5 - Differential gene expression in APP/PS1 transgenic mice:

A) Volcano plots of all coding genes detected by Illumina RNA sequencing for the ACC, CA1 and DG of 1.5 (yellow), 4 (red), and 8 (blue) months old transgenic mice. Vertical dashed lines resemble the cutoff for $\log _{2}$ foldchange $( \pm 0.5)$ and horizontal dashed lines resemble the cutoff for the adjusted $p$-value $(\leq 0.05)$. Note that differential gene expression in APP/PS1 transgenic mice is prone to upregulation rather than downregulation of genes.

B) Number of up- and downregulated genes for the given age groups and brain regions.

C,E,G) Results from partition around medoids clustering of $\log _{2}$ foldchanges for 7 clusters. Values depicted in each cell represent the respective cluster's medoid of foldchanges for each age group. Downregulation of genes is resembled by blue, upregulation by red boxes.

$\mathbf{D , F}, \mathbf{H})$ Venn diagrams showing the number of common and exclusive differentially expressed genes in the ACC, CA1 and DG of 1.5, 4, and 8 months old wildtype and APP/PS1 transgenic mice. 
PAM analysis was done for 7 clusters to enable one cluster for each possible intersection of shared genes and group-exclusive genes. Figure 4.5C, E, and G (see p.63) shows heatmaps for the medoids retrieved for each respective clustering. With only one exception in the CA1, the deregulation of gene expression is changing in a unidirectional manner. A gene that is deregulated in 4 months old mice is thus likely to be deregulated in the same direction in a later stage as well. Only a single cluster in ACC was identified, were genes are upregulated in 1.5 months old APP/PS1 transgenic mice and not deregulated anymore in older mice. The DG is the only brain regions with more than one cluster of downregulated genes identified.

The findings from the PAM analysis also becomes visible in the Venn diagrams shown in figure 4.5D, F, and H (see p.63). The vast majority of deregulated genes is shared between 4 and 8 months old mice, while those genes deregulated during early life are almost exclusive to young mice (ACC: 33 out of 41, CA1: 18/21, DG: 19/22). Only few genes are shared among all age groups due to that exclusivity of genes affected in early life, namely Thy1 (all brain areas), Nipal4 (ACC), Mrc2, and Capn3 (both CA1). Only 22 genes differentially expressed in the ACC of 4 months old mice were not expressed in the ACC of 8 months old mice as well. In the CA1, 78 out of 326 deregulated genes in 4 months old mice were exclusive for 4 months old mice and 7 out of 50 in the DG. These data suggest that the general pattern of molecular deregulation is already set at an age of 4 months and becomes more intense during aging.

1.5 months

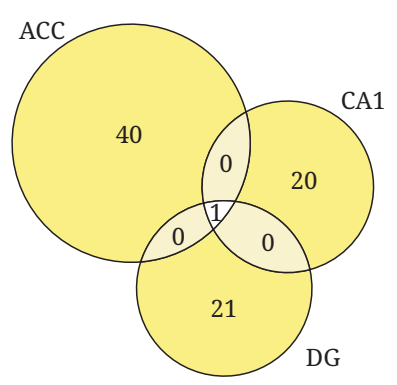

4 months

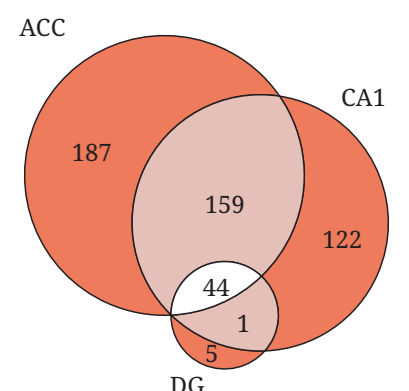

8 months

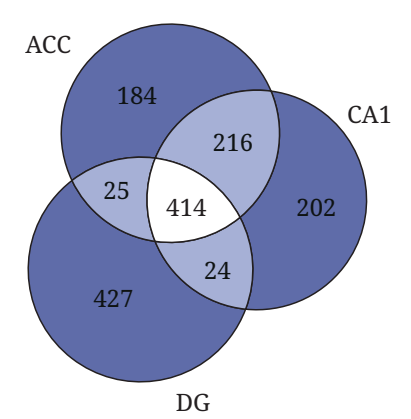

Figure 4.6 - Differentially expressed genes in APP/PS1 mice shared among brain regions:

Venn diagrams for common and brain region selective differentially expressed genes in 1.5 (yellew), 4 (red), and 8 months old (blue) APP/PS1 transgenic vs wildtype mice.

Figure 4.6 shows the number of differentially expressed genes in APP/PS1 transgenic mice that are shared among the three tested brain regions. While only a single gene, namely Thy1, is 
shared among the regions in 1.5 months old mice, the majority of genes is shared in the older mice. Only 5 genes from the DG are selective for that brain region in 4 months old mice while the majority of differentially expressed genes are shared among all three brain regions. In total, the ACC and CA1 of 4 months old mice shared approximately $52-62 \%$ of significant genes respectively. In 8 months old mice, the majority of genes is shared among the ACC, CA1, and DG as well (all: 414, ACC\&DG: 25, ACC\&CA1: 216, CA1\&24: 24). However, the DG only shares approximately half of its differentially expressed genes with the other brain regions. 427 out of 890 genes are exclusive for the DG and the majority of these 427 genes (318) is downregulated. Together with data from the regional comparisons in section 4.1 (see p.56), these data confirm that the DG as a hippocampal sub-region shares less similarity with the CA1 than the two anatomical distant regions CA1 and ACC share among each other.

\begin{tabular}{lccc}
\multicolumn{1}{c}{1.5 months } & 4 months & $\mathbf{0}$ months & \\
\hline ACC & CA1 & DG
\end{tabular}

A
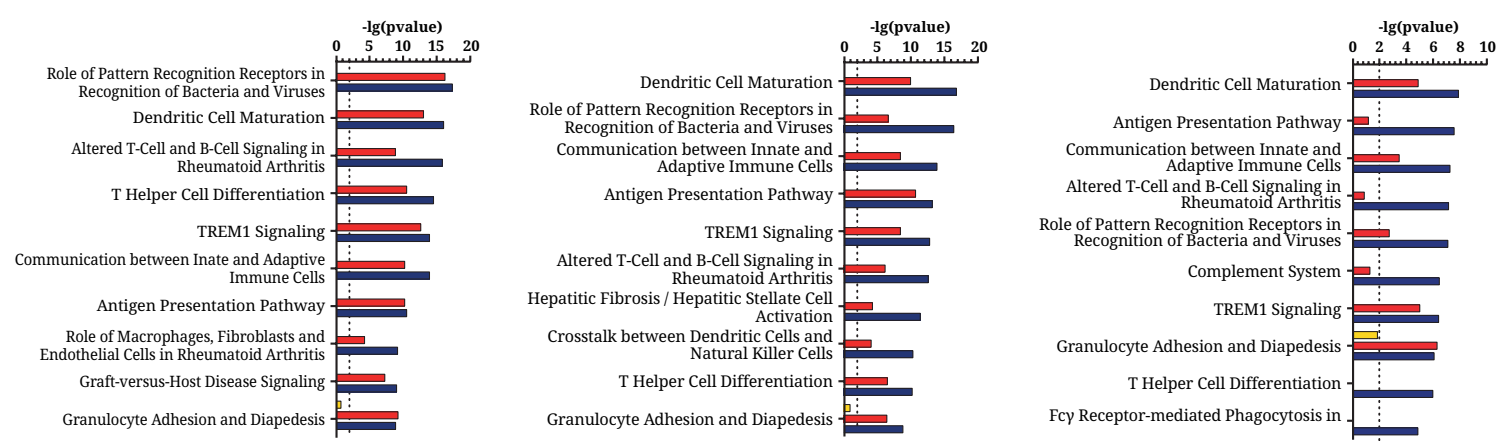

B
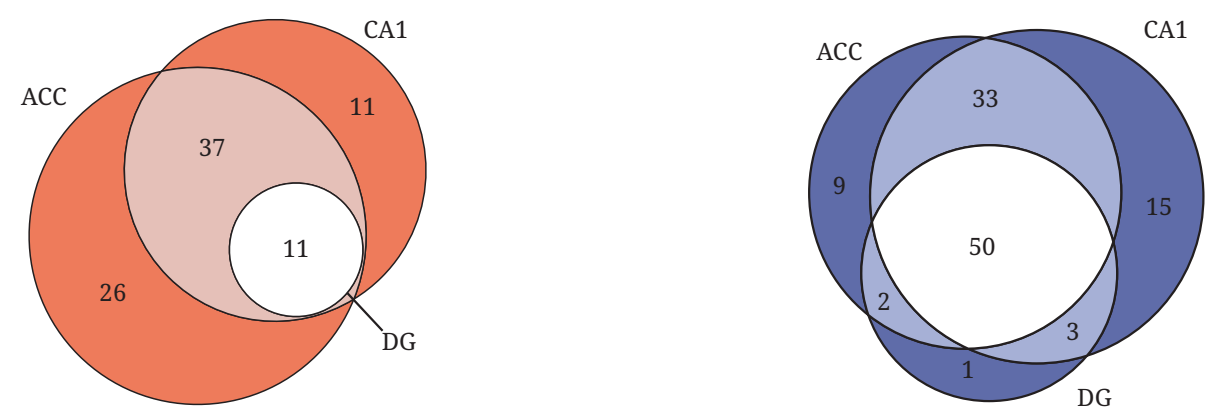

Figure 4.7 - Overrepresented functional pathways in APP/PS1 transgenic mice:

A) Enriched functional pathways in 1.5 (yellow), 4 (red), and 8 (blue) months old APP/PS1 transgenic mice compared to wildtype mice identified by Ingenuity Pathway Analysis. The top 10 pathways identified for 8 months old mice were chosen for plotting. Dotted lines resemble the threshold for significance $(=0.01)$.

B) Number of common and region exclusive significant functional pathways for 4 (red) and 8 months (blue) old mice respectively. 
To bring the deregulation of gene expression during aging in a functional context, I performed functional pathway analysis using Ingenuity. The top 10 significant pathways $(p<0.01)$ identified for the ACC, CA1, and DG of 8 months old APP/PS1 transgenic mice compared to wildtype mice are displayed in figure 4.7 (see p.65). From the top10 pathways of a brain region, the majority (8) is also among the top 10 in the other regions and can be related to immune response, i.e. "Dendritic Cell Maturation", "Role of Pattern Recognition" (innate immune system related), or "TREM1 (Triggering receptor expressed on myeloid cells) Signaling”. By comparing all significant functional pathways in the given set of deregulated genes, the majority of pathways is still shared among all three regions (see figure 4.7B, p.65). While few pathways can be related to metabolism (i.e. "cholesterol biosynthesis", ”Type I Diabetes Mellitus Signaling”), the vast majority of pathways can be linked with immune response mainly resembling upregulation of gene expression. None of the significant pathways can be directly linked to neuronal plasticity.

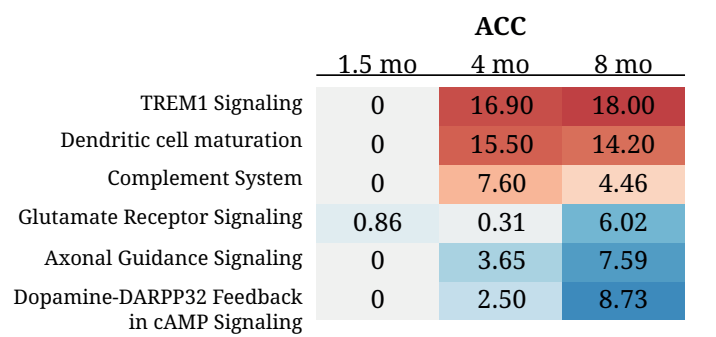

\begin{tabular}{ccc} 
& CA1 & \\
$1.5 \mathrm{mo}$ & $4 \mathrm{mo}$ & $8 \mathrm{mo}$ \\
\hline 1.06 & 10.40 & 20.3 \\
0.71 & 11.30 & 18.6 \\
0 & 9.26 & 6.03 \\
0 & 1.78 & 2.82 \\
1.26 & 1.2 & 6.73 \\
0 & 1.74 & 11.2 \\
\hline
\end{tabular}

\begin{tabular}{ccc}
\multicolumn{3}{c}{ DG } \\
$1.5 \mathrm{mo}$ & $4 \mathrm{mo}$ & $8 \mathrm{mo}$ \\
\hline 0 & 4.58 & 12.40 \\
0 & 5.52 & 14.00 \\
0 & 4.08 & 8.92 \\
0 & 0 & 2.50 \\
1.52 & 0 & 6.12 \\
0 & 0 & 2.67
\end{tabular}

Figure 4.8 - Functional pathways from mildly downregulated genes:

Three functional pathways related to immune response (red) identified for all upregulated genes also including those with a $\mid \log _{2}$ foldchange $\mid<0.5$ and pathways related to neuronal plasticity (blue) identified for all downregulated genes without foldchange cutoff. Values represent the respective - $\lg (\mathrm{p}$-value) of each pathway and comparison. With few exceptions, deregulation of pathways becomes more significant during aging.

Recently published data for another AD mouse model (Gjoneska et al., 2015) suggests a bulk downregulation of genes related to neuronal plasticity upon amyloid pathology. Additionally, since cognitive decline can already be observed at an age of 4 months in APP/PS1 mice as well, some functional pathways were expected to resemble neuronal functioning. Thus, I also analyzed mildly affected genes $\left(\mid \log _{2}\right.$ foldchange $\left.\mid<0.5\right)$ for overrepresented functional pathways (see section 7.2, p.131ff.). Seventeen pathways with a p-value $\leq 0.01$ were found, shared among the ACC, CA1, and DG in 8 months old mice regarding downregulated genes. Some of those pathways can clearly be related to neuronal plasticity, i.e. ”Axonal Guidance Signaling”, ”DopamineDARPP32 Feedback in cAMP Signaling”, and "Glutamate Receptor Signaling”. Figure 4.8 shows 
the $\lg (\mathrm{p}$-value) for the previously mentioned plasticity related pathways and three arbitrary chosen immune response related pathways ("Complement System”, ”Dendritic Cell Maturation”, "TREM1 Signaling”) for each given age group and brain region. As it was previously shown for progression of gene expression per se (see figure 4.5, p.63), these pathways also behave in a unidirectional manner. Overrepresented pathways in 4 months old mice become more significant during aging. Among the set of pathways identified for mildly upregulated genes, only a single plasticity-related pathway was found common for all three regions, namely "Reelin Signaling in Neurons". However, more neuronal signaling pathways were found common for the ACC and the CA1, i.e. "Synaptic Long Term Depression”. These data suggest that the neurons in APP/PS1 transgenic mice are only mildly affected in their gene expression by amyloid pathology and that the major effect on gene expression is acting on glial cells functioning in immune response upon amyloid deposition. 
Besides quality control of Illumina sequencing itself, data from RNAseq are usually validated via qPCR to ensure trustful data. Figure 4.9 shows the validation via qPCR for two highly deregulated genes, namely MPEG1 and RUNX1. A similar pattern of deregulation found by RNAseq can be identified for qPCR data as well. Significance analysis with ANOVA and Tukey's multiple comparisons test confirms the findings from RNAseq for both MPEG1 ( $\mathrm{p}_{\text {genotype }}<0.0001 ; \mathrm{p}_{4 v s 8}=$ 0.0092 ) and RUNX1 ( $\left.\mathrm{p}_{\text {genotype }}=0.0001 ; \mathrm{p}_{4 v s 8}=0.0112\right)$.

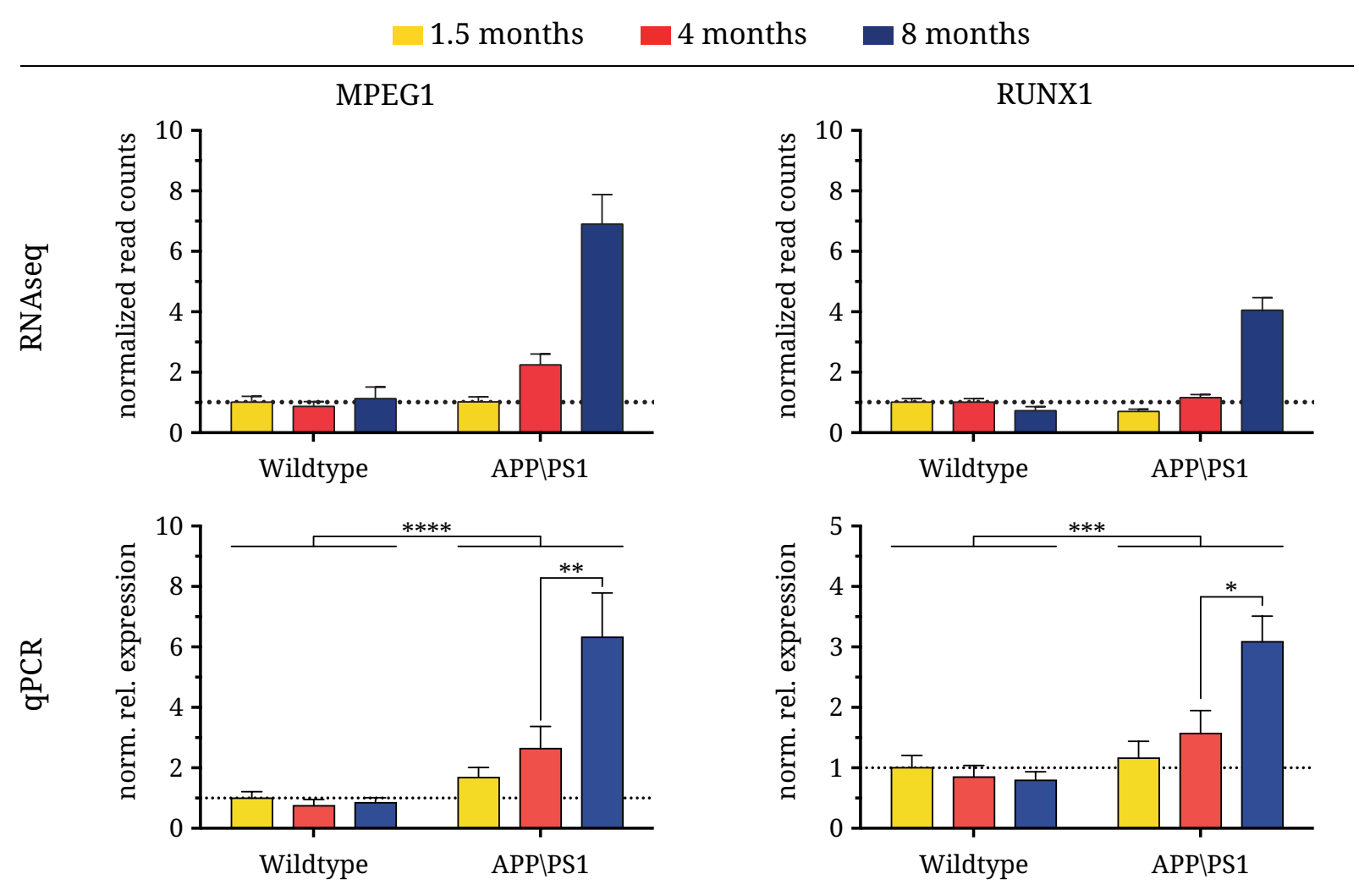

Figure 4.9 - Validation of RNA sequencing via qPCR:

Data from RNAseq (top) and qPCR (bottom) for MPEG1 (left) and RUNX1 (right). The very same RNA samples of 1.5, 4, and 8 months old wildtype and transgenic mice were used for both RNAseq and qPCR. Data from qPCR was tested for significance using 2-way ANOVA for group effects and Tukey's multiple comparison test for time point comparisons. Significant differences from RNAseq could be validated for both MPEG1 $\left(\mathrm{p}_{\text {genotype }}<0.0001 ; \mathrm{p}_{4 v s 8}=0.0092\right)$ and RUNX1 $\left(\mathrm{p}_{\text {genotype }}=0.0001 ; \mathrm{p}_{4 v s 8}=0.0112\right)$. Error bars resemble the standard error of means. 


\subsection{Differential gene expression during aging}

\subsubsection{Healthy aging in wildtype mice}

Gene expression analysis from APP transgenic mice suggest that the effect of the transgene on gene expression is already severe at 4 months of age. It is mainly driven by immune response related mechanisms and deteriorate during aging. By analyzing transcriptional changes during aging in both APP transgenic and wildtype mice, I aimed to shed some more light on the molecular mechanisms underlying the cognitive decline in those mice. To do so, I first investigated aging in wildtype mice to see how gene expression develops during healthy aging. These data were analyzed similarly to the previously described ones.

Figure 4.10A (see p.70) shows volcano plots of early-life- (1.5 vs 4 months, green) and mid-lifeaging ( 4 vs 8 months, purple) in wildtype mice. For the ACC and CA1, the majority of genes with an adjusted p-value below the given threshold of 0.05 is only mildly differentially expressed. The DG shows a broader pattern of differential gene expression as it could already be observed when comparing the different genotypes. In general, gene expression in wildtype mice across different ages does not show a universal trend towards either up- or downregulation in all age comparisons and brain regions.

While almost an equal number of genes is up- and downregulated in the ACC of 1.5 vs 4 months old mice (155 up, 153 down), 582 genes are downregulated in 4 vs 8 months old mice compared 222 that are upregulated (see figure 4.10B, p.70). In the CA1, only 15\% of the significant genes are upregulated in early-life aging. The number of differentially expressed genes during aging is going down to 267 (67 up, 200 down) in the CA1. Compared to the ACC and CA1, the DG is only mildly affected in regard to gene expression and without any tendency for up- or downregulation. In 1.5 vs 4 months old mice, 153 genes were up- and 179 genes downregulated and the number of significant as well as their ratio of up- vs downregulation remains stable. 


\section{5 vs 4 months 4 vs 8 months}

ACC CA1 DG

A
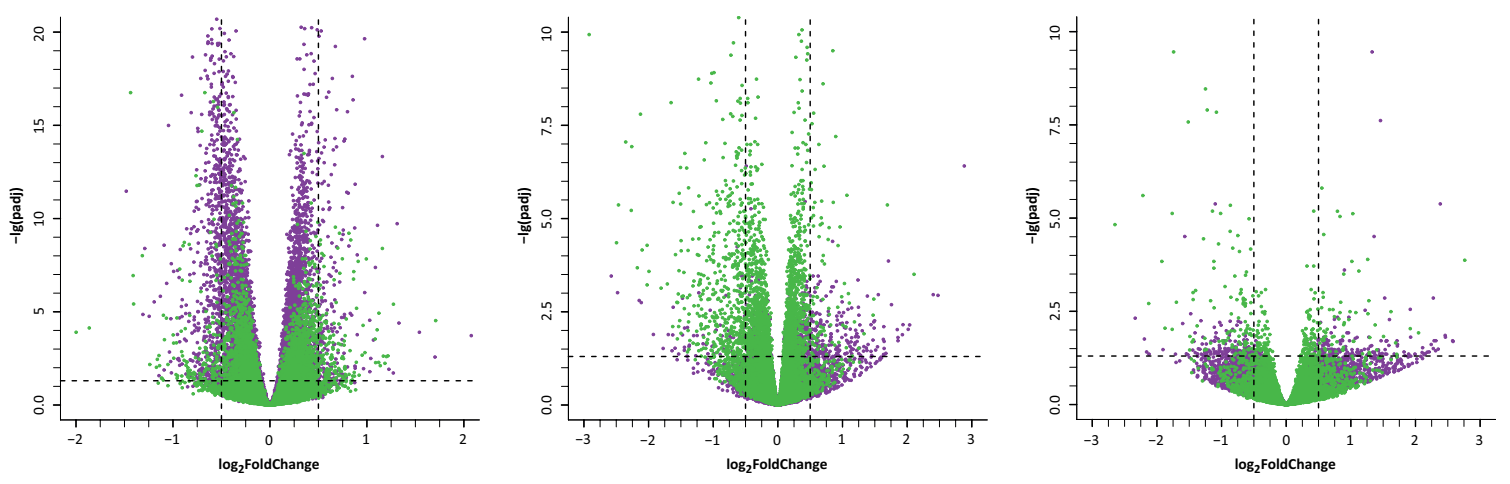

B
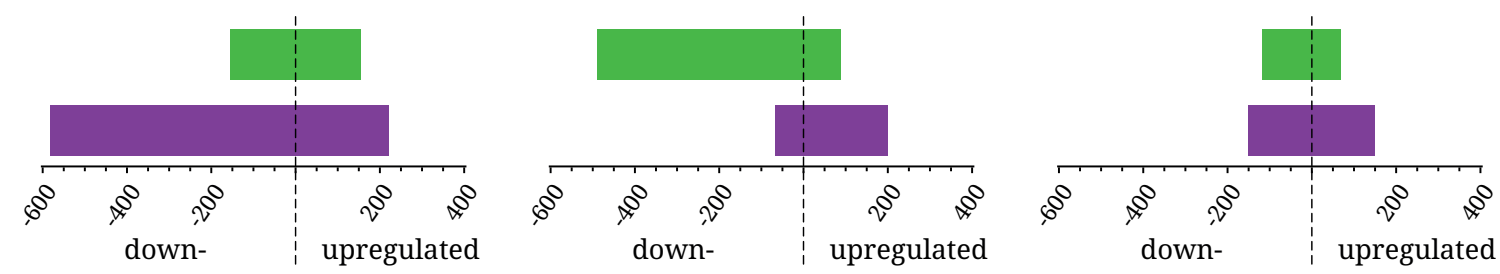

C

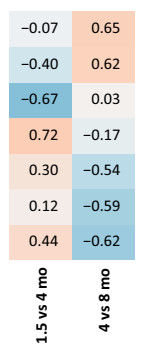

D

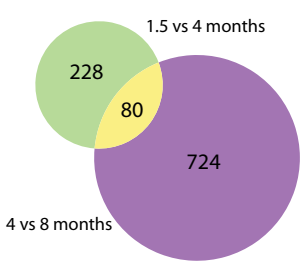

$\mathbf{E}$

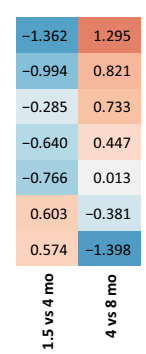

$\mathbf{F}$

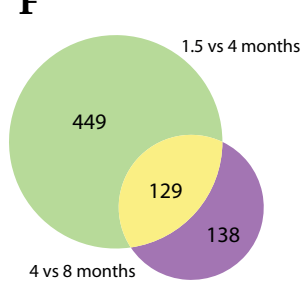

$\mathbf{H}$

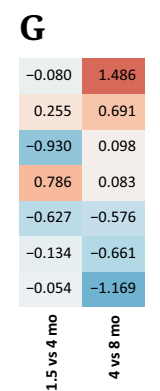

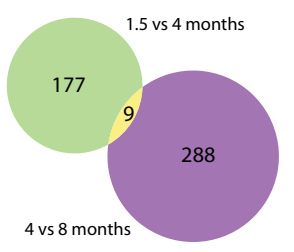

Figure 4.10 - Differential gene expression in aging wildtype mice:

A) Volcano plots of all coding genes detected by Illumina RNA sequencing for the ACC, CA1 and DG of 1.5 vs 4 (green) and 4 vs 8 (purple) months old wildtype mice. Vertical dashed lines resemble the cutoff for $\log _{2}$ foldchange $( \pm 0.5)$ and horizontal dashed lines resemble the cutoff for the adjusted p-value $(\leq 0.05)$. Note that differential gene expression in wildtype mice is slightly tended towards downregulation during aging.

B) Number of up and downregulated genes for the given age groups and brain regions.

C,E,G) Results from partition around medoids clustering of $\log _{2}$ foldchanges for 7 clusters. Values depicted in each cell represent the respective cluster's medoid of fold changes for each age comparison. Downregulation of genes is resembled by blue, upregulation by red boxes.

$\mathbf{D , F}, \mathbf{H})$ Venn diagrams showing the number of common and exclusive differentially expressed genes in the ACC, CA1 and DG of 1.5 vs 4 and 4 vs 8 months old wildtype mice. 
PAM analysis of aging-related differential gene expression in wildtype mice revealed that gene expression is not following a unidirectional pattern as it was observed for the comparison of wildtype vs transgenic mice (see figure 4.10C, E, and G, p.70). Three different kinds of clusters were identified: genes that are not yet affected in early-life aging but in mid-life aging or vice versa; genes that are downregulated in 4 months old mice compared to 1.5 months old mice but upregulated in 8 months old mice or vice versa; and genes that are downregulated in both aging comparisons. The only cluster of the latter kind was observed in the DG. The majority of genes differentially expressed in the CA1 reverse their direction of deregulation during aging, matching the shift from down- to upregulation seen in figure 4.10B (see p.70).
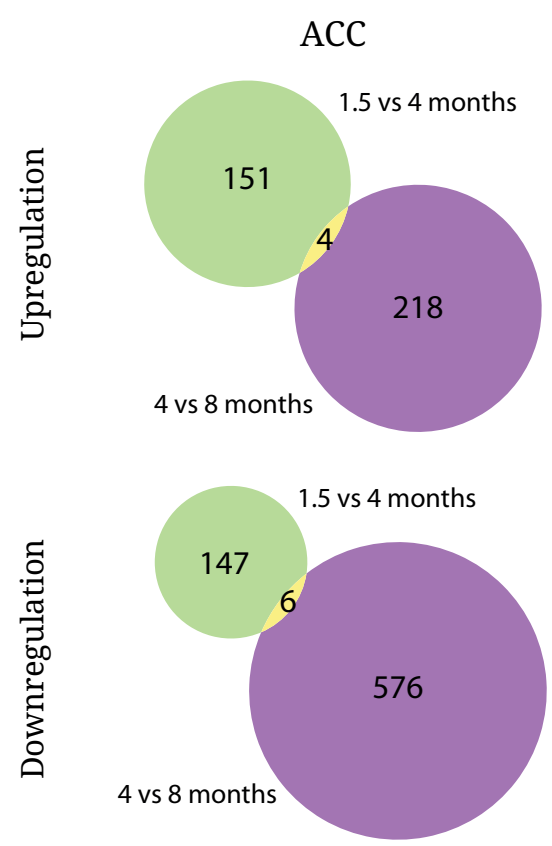

CA1
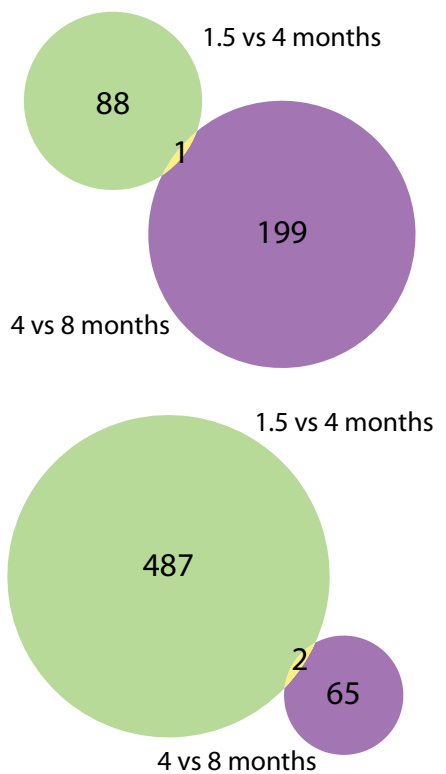
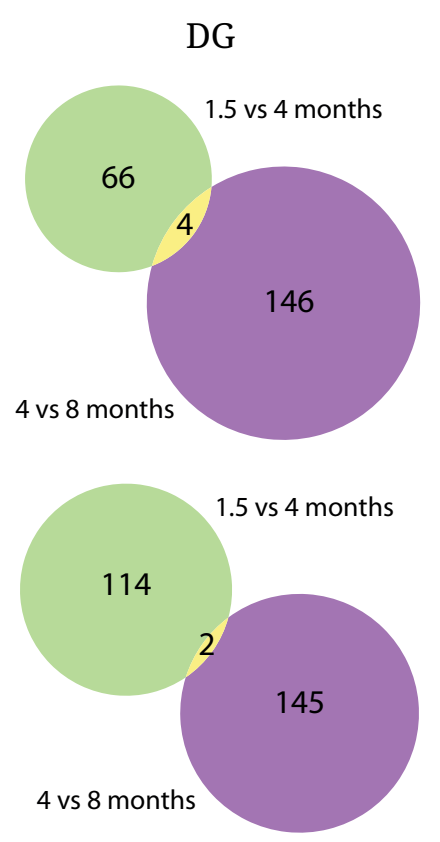

Figure 4.11 - Common up- and downregulated genes during aging in wildtype mice:

Venn diagrams for up- (top) and downregulated (bottom) genes shared between early-life and mid-life aging in wildtype mice. Only genes deregulated in the same direction are plotted.

The findings from PAM analysis are also reflected by the Venn diagrams for 1.5 vs 4 and 4 vs 8 months old mice showing that only a small fraction of genes is shared among the aging comparisons in the ACC (80 from 308 in early-life and 804 in mid-life aging) (see figure 4.10D, F, and H, p.70). This effect is even stronger in the DG, where only 9 genes out of 186 and 297 respectively are shared, partially resembling the cluster of unidirectional downregulated genes. Almost half of the genes differentially expressed in the CA1 of 4 vs 8 months old mice (129 out of 267) is also 
differentially expressed in early-life aging. However, data from PAM analysis already suggested that most genes are likely to develop in opposite directions during aging progression. Figure 4.11 (see p.71) confirms these findings. Out of the 80 genes shared between the aging groups in the ACC of wildtype mice, only 4 are upregulated and 6 downregulated in both comparisons. Only 3 genes from both age comparisons are developing in the same direction in the CA1 ( 2 down, 1 up) and in the DG, only 9 genes were shared among the aging groups to begin with. 4 of those are upregulated during aging and 2 are downregulated.

4 vs 8 months

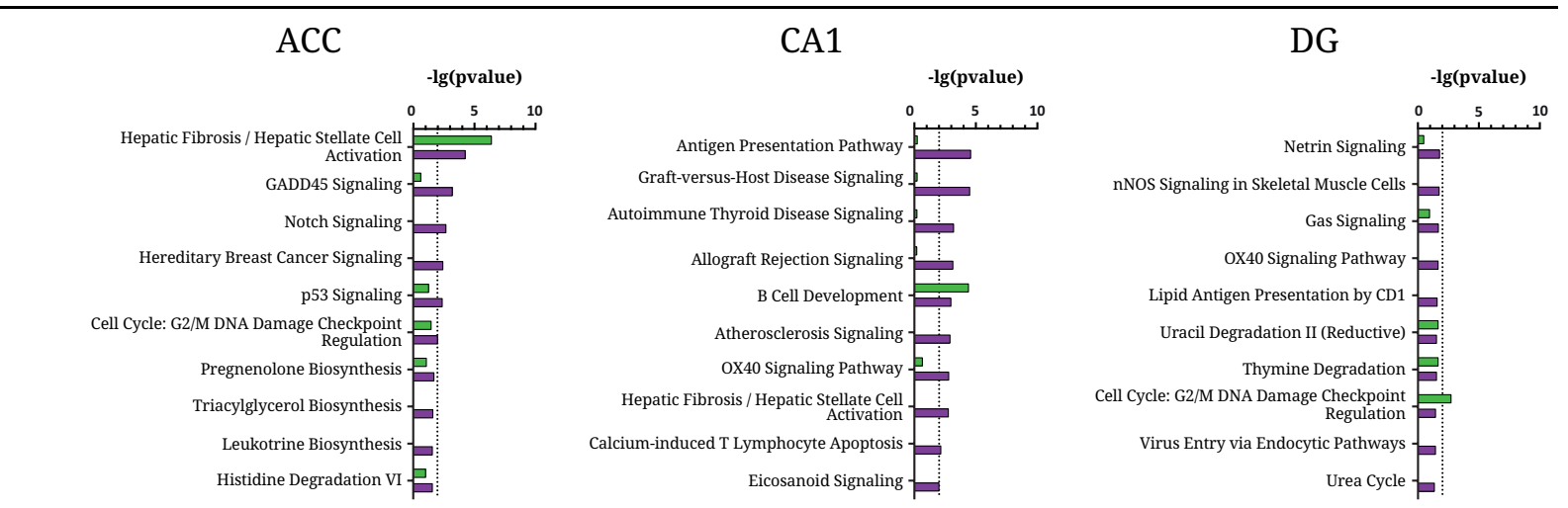

Figure 4.12 - Overrepresented functional pathways in aging wildtype mice:

Overrepresented functional pathways in aging wildtype mice identified by Ingenuity Pathway Analysis. The top 10 pathways identified for 4 vs 8 months old mice were chosen for plotting. Dotted lines resemble the threshold for significance (=0.01).

To identify functional pathways involved in healthy aging, I performed a functional pathway analysis using Ingenuity (see figure 4.12). Only few pathways were significant $(p \leq 0.01)$ and due to the relatively low coverage of genes between the two age comparisons in the respective brain regions, the pathways are exclusive for one of the aging comparisons with few exceptions. Not a single pathway was found to be significant in the DG of 4 vs 8 months old mice. The categories of the few significant functional pathways detected for the other tested brain regions include immune response (i.e. "Antigen Presentation Pathway") and homeostasis or anti-cancer pathways (i.e. "p53 signaling”, ”Cell Cycle: G2/M DNA Damage Checkpoint Regulation”).

As mice from 1.5 to 8 months of age are not expected to develop severe cognitive impairments, these data are fitting to the phenotype of wildtype aging. Still, due to the low number of overrepresented pathways, I ran a pathway analysis on all up- and downregulated genes respectively, 
regardless of the foldchange (see figure 4.13). Due to the relatively weak effect of aging on gene expression in the DG of aging wildtype mice, only few functional pathways were identified even without a cutoff for expression foldchanges. Pathways identified for the DG can be linked to immune response, however, less than 4 genes were matched for each pathway. The ACC and CA1 both show a higher number of differentially expressed genes compared to the DG. Though the categories of functional pathways are quite diverse, the majority of them can be related to neuronal functions (i.e. ”Synaptic Long Term Potentiation”, ”CDK5 Signaling”, ”Axonal Guidance Signaling”).

\section{5 vs 4 months $\quad 4$ vs 8 months}

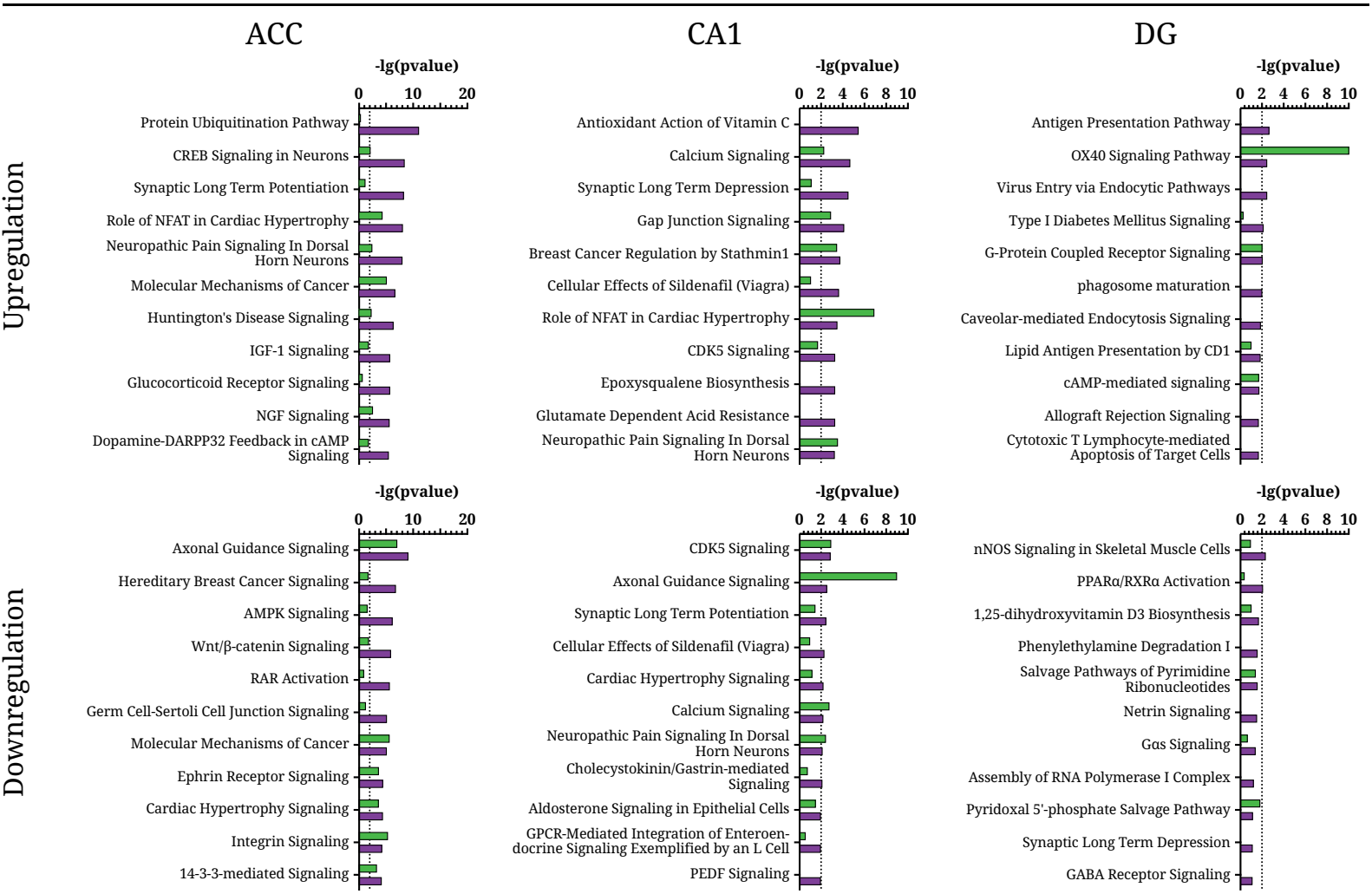

Figure 4.13 - Functional pathways from mildly deregulated genes in aging wildtype mice:

Overrepresented functional pathways in aging wildtype mice identified by Ingenuity Pathway Analysis for up- and downregulated genes separately. All genes with an adjusted p-value $\leq 0.05$ were considered, regardless of their respective foldchange. The top 10 pathways identified for 4 vs 8 months old mice were chosen for plotting. Dotted lines resemble the threshold for significance $(=0.01)$.

Figure 4.14 (see p.74) shows the number of significant pathways that are shared between the brain regions. Approximately 35-60\% of all pathways identified for the CA1 of up- or downregulated genes in early- or mid-life aging were also identified for the ACC of the same comparison. 
No clear separation of pathway categories regarding up- and downregulation of genes can be observed, suggesting that the differential gene expression resembles a shift from post-natal developmental to mature neuronal pathways rather than a deregulation of these.

\section{5 vs 4 months old}
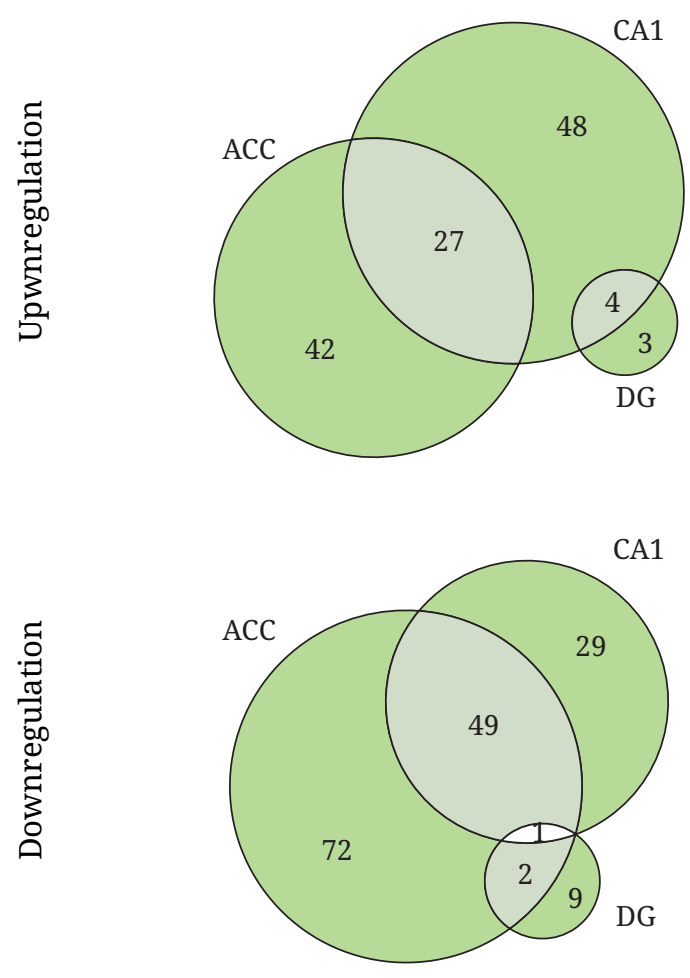

4 vs 8 months old
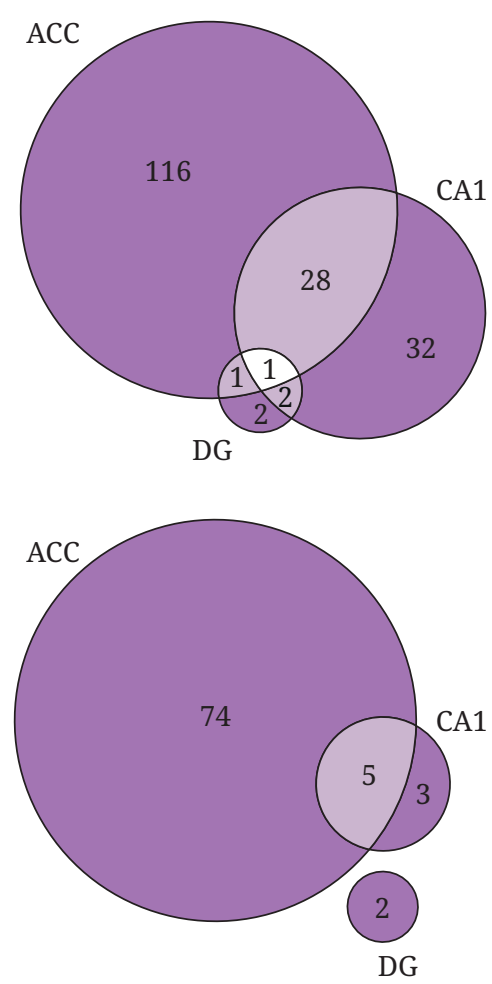

Figure 4.14 - Common functional pathways from mildly deregulated genes in aging wildtype mice: Number of overrepresented functional pathways identified for $1.5 \mathrm{vs} 4$ (left) and 4 vs 8 (right) months old wildtype mice, common for the ACC, CA1, and DG. Pathway analysis was based on all genes with an adjusted $\mathrm{p}$-value $\leq 0.05$, regardless of the respective foldchange. 


\subsubsection{Pathological aging in APP/PS1 transgenic mice}

\section{5 vs 4 months 4 vs 8 months}

ACC

A

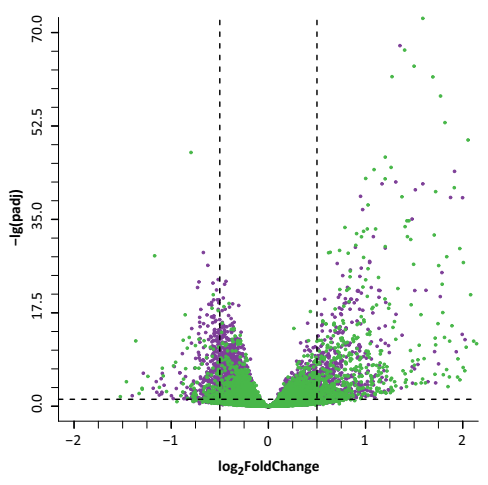

B

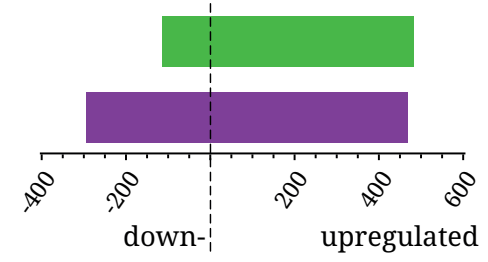

C

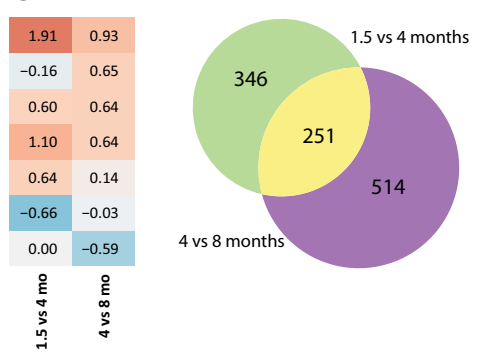

CA1
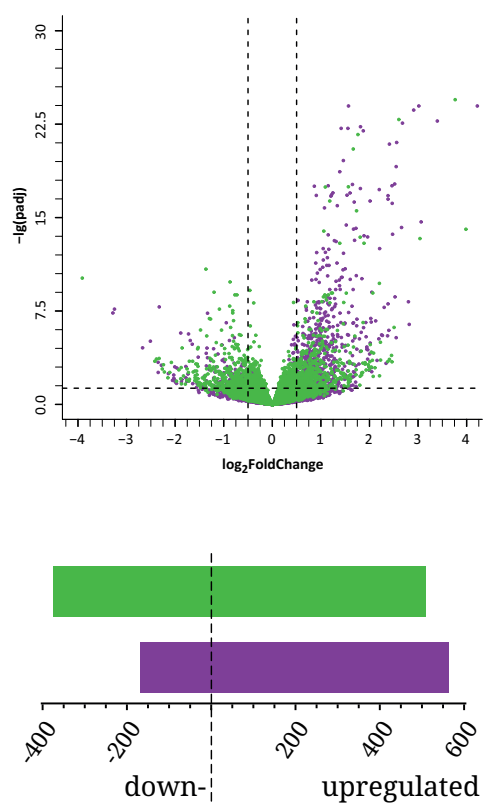

E
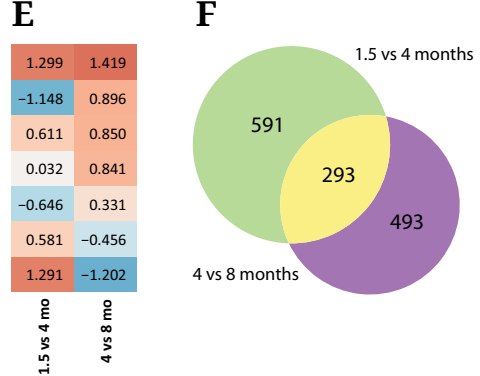

DG
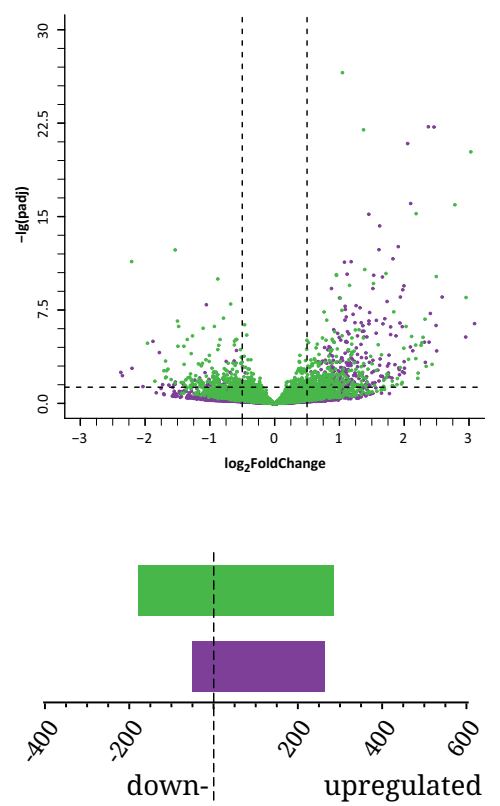

G
$\mathbf{H}$

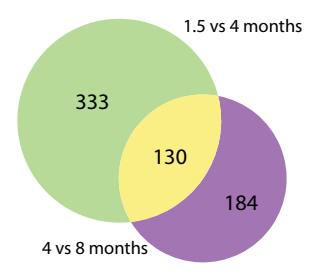

Figure 4.15 - Differential gene expression in aging APP/PS1 mice:

A) Volcano plots of all coding genes detected by Illumina RNA sequencing for the ACC, CA1 and DG of 1.5 vs 4 (green) and 4 vs 8 (purple) months old APP/PS1 transgenic mice. Vertical dashed lines resemble the cutoff for $\log _{2}$ foldchange $( \pm 0.5)$ and horizontal dashed lines resemble the cutoff for the adjusted p-value $(\leq 0.05)$. Note that differential gene expression in transgenic mice is slightly tended towards upregulation during aging.

B) Number of up and downregulated genes for the given age groups and brain regions.

C,E,G) Results from partition around medoids clustering of $\log _{2}$ foldchanges for 7 clusters. Values depicted in each cell represent the respective cluster's medoid of fold changes for each age comparison. Downregulation of genes is resembled by blue, upregulation by red boxes.

$\mathbf{D}, \mathbf{F}, \mathbf{H})$ Venn diagrams showing the number of common and exclusive differentially expressed genes in the ACC, CA1 and DG of 1.5 vs 4 and 4 vs 8 months old transgenic mice. 
Gene expression profiles from aging APP/PS1 transgenic mice are similar to the comparisons of wildtype and transgenic mice (see figure 4.15A, p.75). Genes tend towards upregulation of their expression and the DG shows the mildest deregulation of gene expression of all three regions. During early-life aging (1.5 vs 4 months), 482 genes are upregulated in the ACC compared to 115 downregulated ones and the number of downregulated genes is increasing to 296 in mid-life aging (4 vs 8 months) (see figure 4.15B, p.75). In the CA1 of 1.5 vs 4 months old mice, 509 genes are upregulated and 375 downregulated compared to 563 upregulated and 169 downregulated genes in 4 vs 8 months old transgenic mice. With 463 significant genes in total, the number of deregulated genes in the DG is relatively small and is decreasing further during aging. The number of differentially expressed genes during aging in transgenic mice is only slightly different than those observed for wildtype aging, though the expression profile with its tendency towards upregulation highlights the diverse effect of aging in those mice.

1.5 vs 4 months 4 vs 8 months

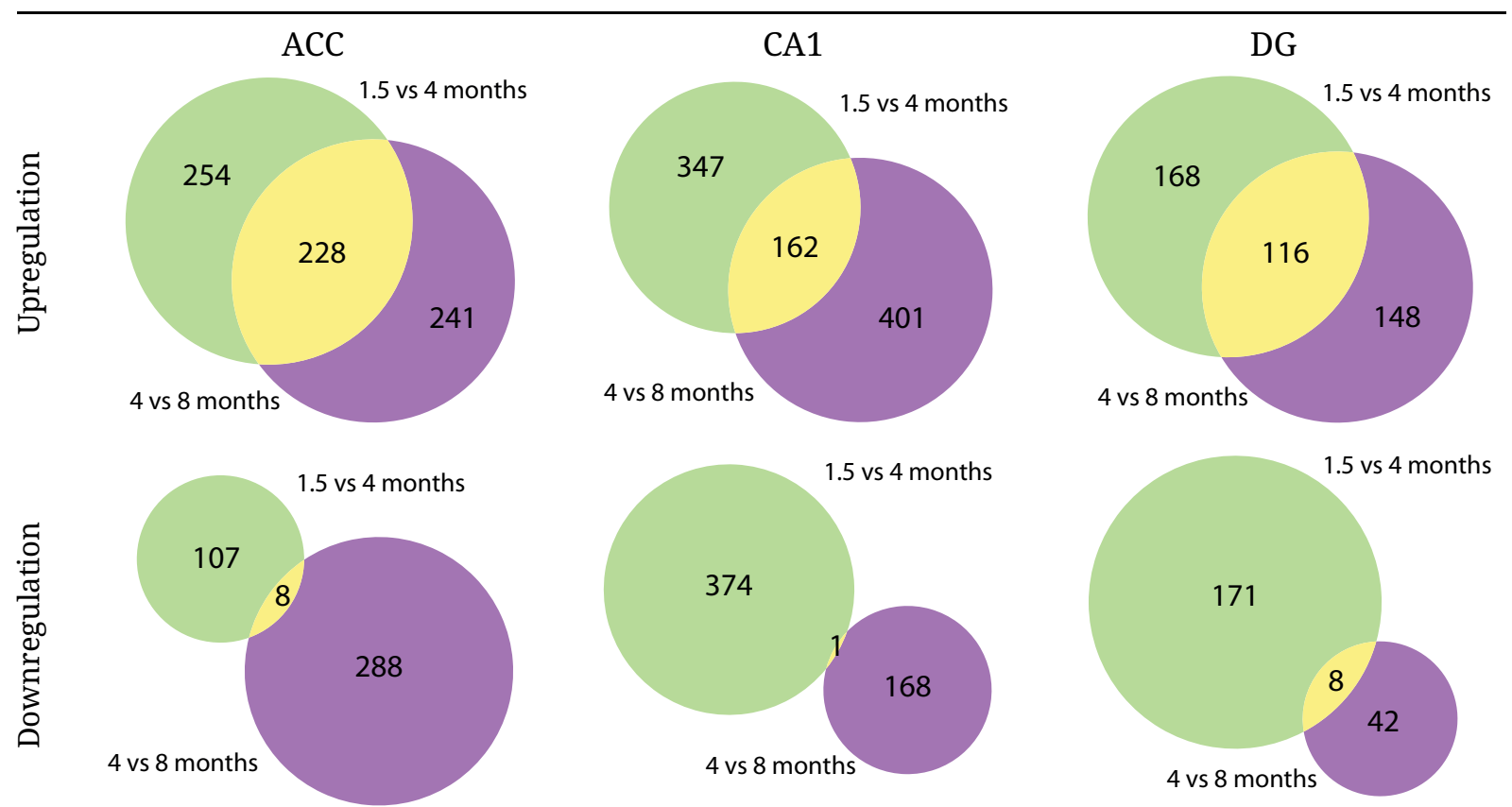

Figure 4.16 - Common up- and downregulated genes during aging in transgenic mice:

Venn diagrams for up- (top) and downregulated (bottom) genes shared between early-life and mid-life aging in transgenic mice. Only genes deregulated in the same direction are plotted.

Figure 4.15C, E, and G (see p.75) shows PAM cluster analysis for aging APP/PS1 transgenic mice. Similar to aging in wildtype mice, genes are not only developing in a unidirectional pattern, 
though gene expression changes in aging transgenic mice seem to be more consistent. The ACC and DG show the most consistent gene expression changes, as most of the genes are either deregulated in the same direction during aging or are affected in either early or late aging only. In contrast, PAM analysis of genes deregulated in the CA1 revealed 4 clusters of genes that are changing the direction of deregulation, thus are downregulated in early-life aging and upregulated in midlife aging or vice versa. Only a single cluster was found for the DG where genes in both earlyand mid-life aging are significantly downregulated.

A substantial number of genes is commonly differentially expressed in both aging groups for the respective brain regions (see figure 4.15D, F, and H, p.75). Approximately $35 \%$ of the deregulated genes for either age comparison and brain region were shared with the respective other aging comparison. As it is shown in figure 4.16 (see p.76), the majority of those shared genes are actually deregulated in a unidirectional pattern, except from genes in the CA1. From all 293 genes that are commonly differentially expressed during early-life and mid-life aging, only 163 (162 up, 1 down) are developing in the same direction. This relatively low coverage in the CA1 confirms the findings from PAM clustering (see figure 4.15C, E, and G, p.75). In the ACC and DG, 236 out of 251 and 124 out of 130 genes are unidirectionaly expressed respectively.

As it was already shown for transgenic vs wildtype mice, functional pathway analysis for aging transgenic mice results almost exclusively in immune response related pathways, i.e. ”Dendritic Cell Maturation” or "TREM1 Signaling” (see figure 4.17A, p.78). All top10 significant pathways (p $\leq 0.1$ ) identified for the given brain regions are overrepresented in both mid-age and early-life aging. The majority of significant pathways is shared among the ACC, CA1, and DG (see figure 4.17B, p.78). While the most significant pathways in 1.5 vs 4 months old mice were found in the ACC (64 compared to 21 in the CA1 and 30 in the DG), the ACC is the region with the least overrepresented pathways in 4 vs 8 months old mice (ACC: 43, CA1: 67, DG: 71), though it is the region with the highest number of differentially expressed genes (see figure 4.15B, p.75).

In healthy aging, both mildly up- and downregulated genes during aging were involved in neuronal plasticity related pathways (see figure 4.13, p.73). Here, I performed a similar pathway analysis based on mildly differentially expressed genes of both directions separately (see figure 4.18, p.79). The top 10 pathways revealed for upregulated genes are again exclusively related to immune response. From all significant pathways common between the brain regions, the only 


\section{5 vs 4 months 4 vs 8 months}

\begin{tabular}{|c|c|c|c|c|c|}
\hline \multicolumn{2}{|l|}{$\mathrm{ACC}$} & \multicolumn{2}{|l|}{ CA1 } & \multicolumn{2}{|l|}{ DG } \\
\hline & $-\lg ($ pvalue $)$ & \multicolumn{2}{|r|}{$-\lg ($ pvalue) } & & $-\lg ($ pvalue $)$ \\
\hline & لعسبس $\quad 20$ & & لمس & & $\begin{array}{lllll}0 & 5 & 10 & 15 & 20 \\
& 5 & & & \end{array}$ \\
\hline Dendritic Cell Maturation & $\begin{array}{lll} \\
\end{array}$ & $\begin{array}{l}\text { Role of Pattern Recognition Receptors in } \\
\text { Recognition of Bacteria and Viruses }\end{array}$ & 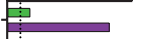 & Antigen Presentation Pathway & : \\
\hline $\begin{array}{l}\text { Altered T-Cell and B-Cell Signaling in } \\
\text { Rheumatoid Arthritis }\end{array}$ & $\begin{array}{l:l} \\
\end{array}$ & $\begin{array}{l}\text { Communication between Innate and } \\
\text { Adaptive Immune Cells }\end{array}$ & \begin{tabular}{ccc}
$\vdots$ \\
\hdashline
\end{tabular} & $\begin{array}{l}\text { Role of Pattern Recognition Receptors in } \\
\text { Recognition of Bacteria and Viruses }\end{array}$ & 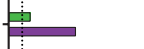 \\
\hline TREM1 Signaling & : & TREM1 Signaling - & 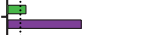 & TREM1 Signaling - & - \\
\hline $\begin{array}{r}\text { Communication between Inate and } \\
\text { Adaptive Immune Cells }\end{array}$ & \begin{tabular}{|l} 
\\
\end{tabular} & Antigen Presentation Pathway & $\begin{array}{l:l} \\
\end{array}$ & Systemic Lupus Erythematosus Signaling & 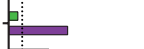 \\
\hline Graft-versus-Host Disease Signaling - & : & Dendritic Cell Maturation - & 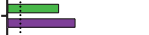 & Dendritic Cell Maturation & 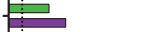 \\
\hline $\begin{array}{l}\text { Role of Pattern Recognition Receptors in } \\
\text { Recognition of Bacteria and Viruses }\end{array}$ & 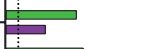 & $\begin{array}{l}\text { Altered T-Cell and B-Cell Signaling in } \\
\text { Rheumatoid Arthritis }\end{array}$ & \begin{tabular}{llll} 
\\
\hdashline
\end{tabular} & $\begin{array}{l}\text { Communication between Innate and } \\
\text { Adaptive Immune Cells }\end{array}$ & 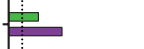 \\
\hline Antigen Presentation Pathway & 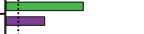 & Leukocyte Extravasation Signaling - & 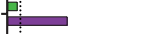 & Allograft Rejection Signaling & $\square$ \\
\hline Complement System & 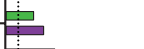 & $\begin{array}{r}\text { Hepatic Fibrosis / Hepatic Stellate Cell } \\
\text { Activation }\end{array}$ & $\begin{array}{lll} \\
\end{array}$ & Graft-versus-Host Disease Signaling - & $\square$ \\
\hline B-Cell Development & - & Graft-versus-Host Disease Signaling . & 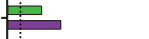 & Autoimmune Thyroid Disease Signaling & 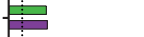 \\
\hline Allograft Rejection Signaling & 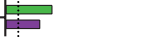 & Systemic Lupus Erythematosus Signaling & ים & Complement System & 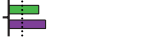 \\
\hline
\end{tabular}

B
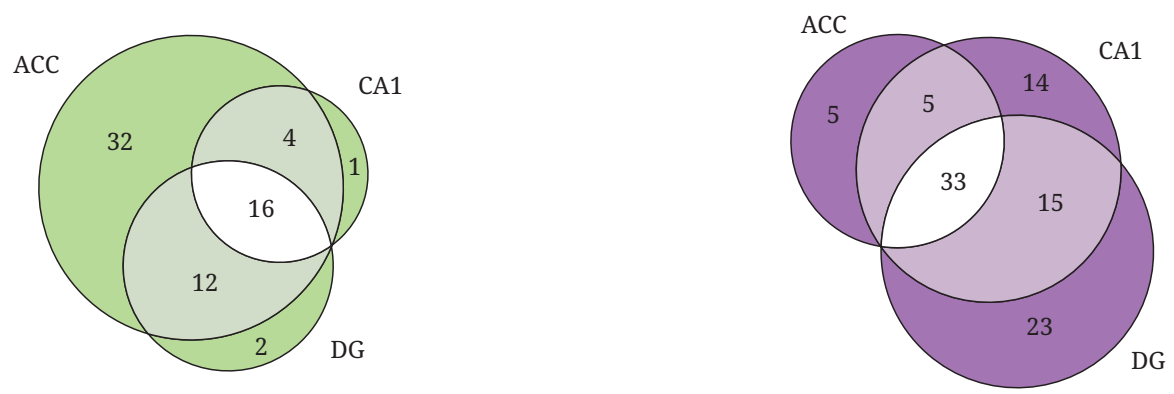

Figure 4.17 - Overrepresented functional pathways in aging transgenic mice:

A) Overrepresented functional pathways in aging APP/PS1 transgenic mice identified by Ingenuity Pathway Analysis. The top 10 pathways identified for 4 vs 8 months old mice were chosen for plotting. Dotted lines resemble the threshold for significance $(=0.01)$.

B) Venn diagrams showing the number of common and region exclusive significant functional pathways for 1.5 vs 4 (green) and 4 vs 8 (purple) months old transgenic mice respectively.

pathway related to neuronal functioning is "Reelin Signaling in Neurons" (see figure 4.19, p.79), while quite a number of neuronal function related pathways were found for all brain regions in healthy animals. Additionally, a single neuronal function related pathways can be found restricted to the ACC namely "Synaptic Long Term Potentiation”. In contrast to upregulated gene expression, analysis for downregulated genes revealed some neuronal functioning related pathways for both age comparisons and all brain regions, i.e. ”Axonal Guidance Signaling”, ”Synaptic Long Term Potentiation” or "Synaptic Long Term Depression”. Consequently, genes from transgenic and wildtype mice do only partially overlap in all aging comparisons and tested brain regions (see figure 4.20, p.80). The majority of genes is uniquely significant in either transgenic or wildtype mice during aging. 


\section{5 vs 4 months $\quad 4$ vs 8 months}

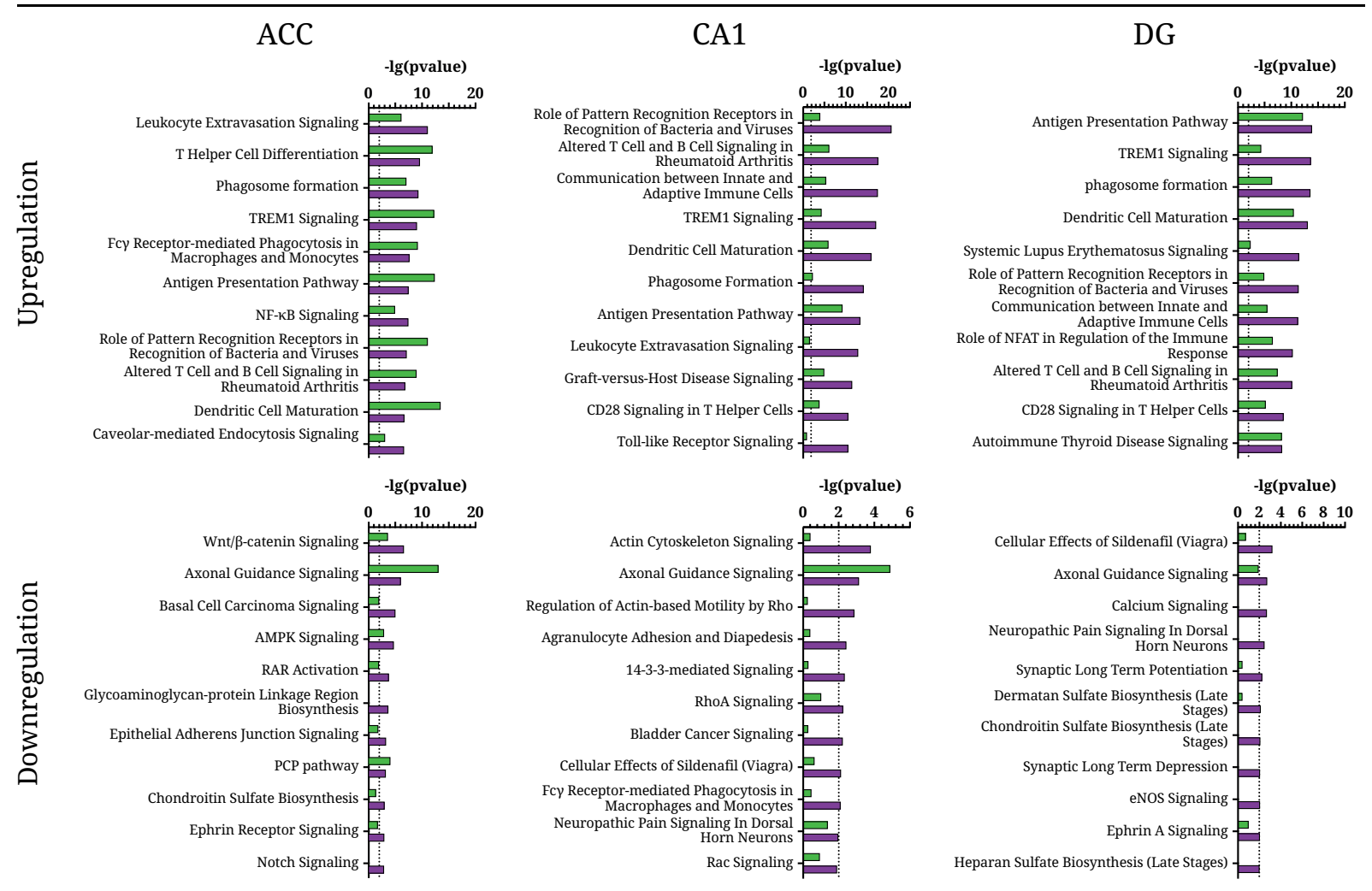

Figure 4.18 - Functional pathways from mildly deregulated genes in aging transgenic mice:

Overrepresented functional pathways in aging transgenic mice identified by Ingenuity Pathway Analysis for up- and downregulated genes separately. All genes with an adjusted p-value $\leq 0.05$ were considered, regardless of their respective foldchange. The top 10 pathways identified for 4 vs 8 months old mice were chosen for plotting. Dotted lines resemble the threshold for significance $(=0.01)$.

\begin{tabular}{|c|c|c|c|c|c|c|}
\hline & \multicolumn{2}{|c|}{ ACC } & \multicolumn{2}{|c|}{ CA1 } & \multicolumn{2}{|c|}{ DG } \\
\hline & 1.5 vs $4 \mathrm{mo}$ & 4 vs $8 \mathrm{mo}$ & 1.5 vs $4 \mathrm{mc}$ & 4 vs $8 \mathrm{mo}$ & 1.5 vs $4 \mathrm{mo}$ & 4 vs $8 \mathrm{mo}$ \\
\hline TREM1 Signaling & 12.20 & 8.98 & 4.27 & 17.00 & 4.31 & 13.60 \\
\hline Dendritic cell maturation & 13.40 & 6.63 & 5.87 & 15.90 & 10.40 & 13.00 \\
\hline Complement System & 5.17 & 4.11 & 5.17 & 8.26 & 6.70 & 7.80 \\
\hline Reelin Signaling in Neurons & 1.72 & 3.00 & 1.87 & 3.30 & 0.80 & 6.18 \\
\hline Glutamate Receptor Signaling & 2.12 & 1.83 & 2.01 & 1.39 & 0.32 & 1.89 \\
\hline Axonal Guidance Signaling & 13.00 & 5.97 & 4.87 & 3.12 & 1.91 & 2.71 \\
\hline $\begin{array}{r}\text { Synaptic Long Term } \\
\text { Potentiation }\end{array}$ & 4.44 & 2.50 & 1.83 & 1.13 & 0.40 & 2.25 \\
\hline
\end{tabular}

Figure 4.19 - Functional pathways from mildly downregulated genes in transgenic mice:

Three functional pathways related to immune response (red) identified for all upregulated genes also including those with a $\mid \log _{2}$ foldchange $\mid<0.5$ and pathways related to neuronal plasticity (blue) identified for all downregulated genes without foldchange cutoff. Values represent the respective -lg(p-value) of each pathway and comparison. With few exceptions, pathways become more significant during aging. 


\section{5 vs 4 months $\quad 4$ vs 8 months}

\section{ACC}

CA1

DG
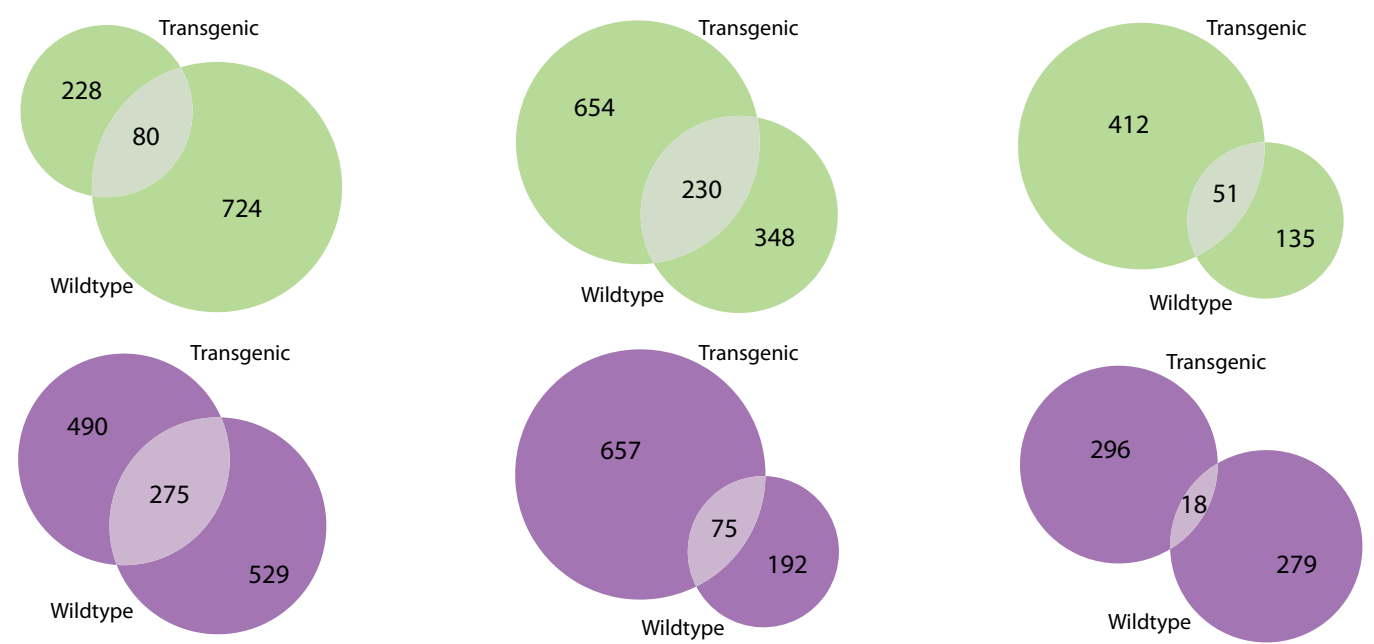

B
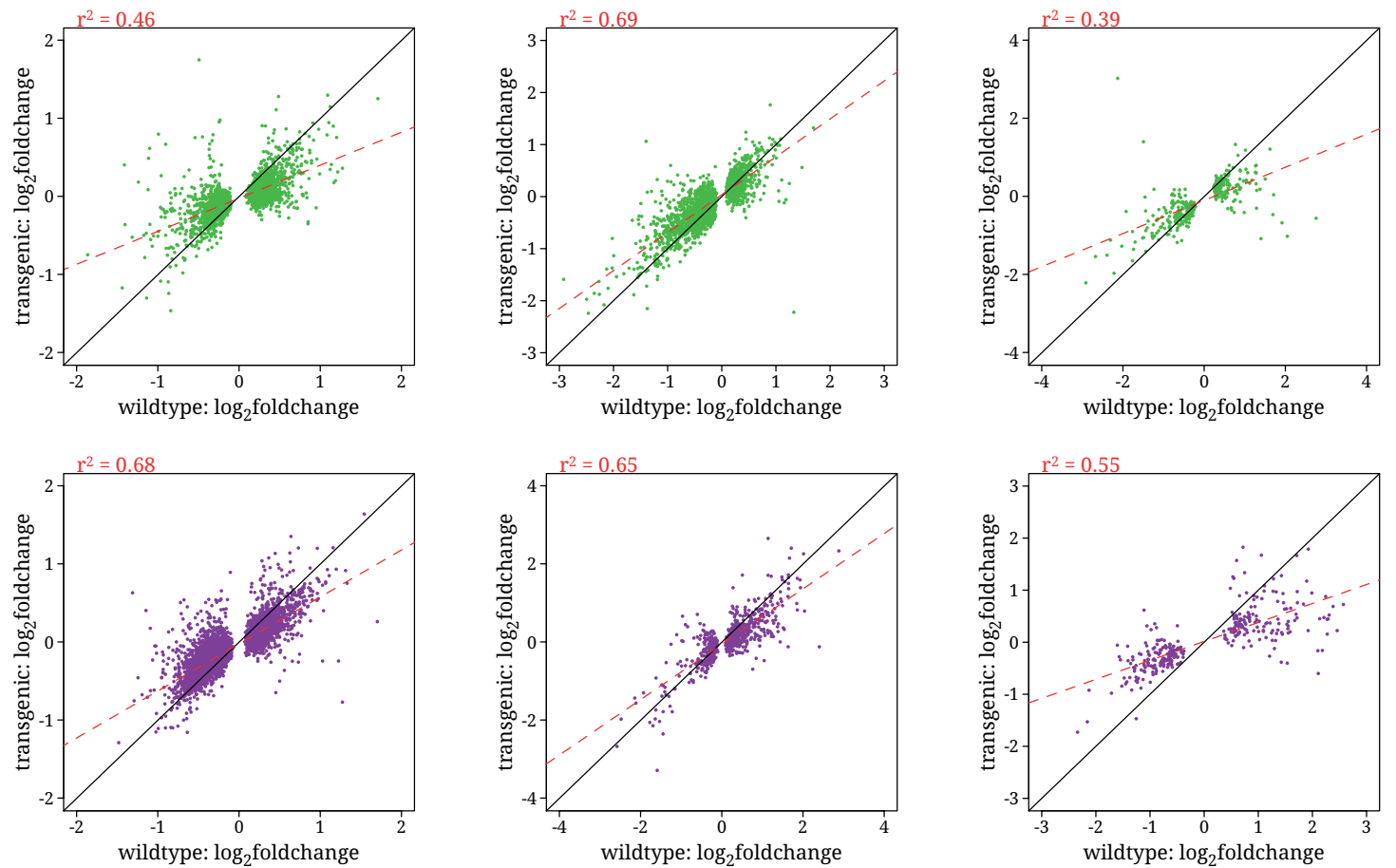

Figure 4.20 - Overlaps in gene expression among wildtype and transgenic aging mice:

A) Venn diagrams for common differentially expressed genes of 1.5 vs 4 (green) and 4 vs 8 (purple) months old wildtype and transgenic mice.

B) Correlation between the foldchange of all significant genes in wildtype mice and the respective foldchange in transgenic mice. All genes with an adjusted p-value $\leq 0.05$ were considered regardless of foldchange. Black lines resemble an ideal correlation of $r=1$. A linear regression is shown for each single comparison by a dashed red line and the computed $\mathrm{r}^{2}$ is depicted ontop of each plot. A global trend can be observed where expression of genes differentially expressed during wildtype aging is less plastic in transgenic mice. 
When calculating the adjusted p-value using multiple comparison algorithms, the output depends on the total number of bins and their respective values. Thus, non-adjusted p-values of a certain magnitude might lead to a significant adjusted p-value in one comparison but to an insignificant one in another. To rule out that genes which are differentially expressed in healthy aging are not masked artificially by the effect of multiple comparison, I compared the foldchange of all genes that were significant in wildtype mice (including mildly deregulated ones) with the foldchange from transgenic aging (see figure 4.20, p.80). A global trend in gene expression becomes visible as the majority of genes upregulated during aging in wildtype mice are only mildly affected or even downregulated in transgenic mice and vice versa for downregulated genes in wildtype mice.

Taken together, data from young mice (see section 4.2, p.59ff.) and differential gene expression during aging suggest that transgenic mice lose plasticity in neuronal functioning related gene expression, which might partially cause the cognitive decline in those mice.

\subsection{Induction of immune response in APP/PS1 mice}

Data from differential gene expression revealed that the major effects seen in APP/PS1 transgenic mice can be related to immune response. Immune response in the CNS is mainly driven by microglia and astrocytes. To rule out that differential gene expression seen in transgenic mice is only the effect of a gliosis, I evaluated the ratio between neuronal and non-neuronal cells in the given set of mice and performed confocal imaging using neuronal, microglial and astrocytic marker. As it is shown in figure 4.21A (see p.82), the ratio of neuronal:non-neuronal cells is not changing significantly between transgenic and wildtype mice. Confocal imaging further indicate that the significant changes observed in APP/PS1 mice is rather a result from glial activation than an induction of cell number as no obvious differences could be observed between wildtype and transgenic mice, as it is exemplary shown for 4 months old mice in figure 4.22B (see p.82). 
ACC

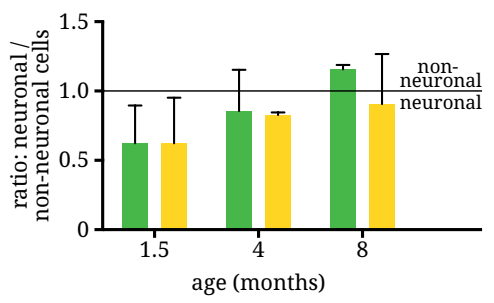

Wildtype APP/PS1

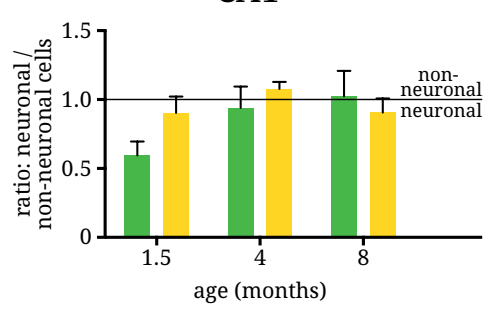

DG

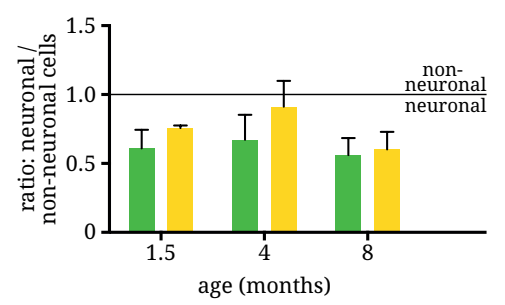

Figure 4.21 - Neural cell counts from transgenic and wildtype mice:

Ratio of neuronal over non-neuronal cells FACS-sorted for epigenetic analysis. No significant differences between wildtype (green) and transgenic (yellow) mice can be observed at the given sample size $(n=2-5$, each $n$ resembles a pool of 3 mice in minimum).
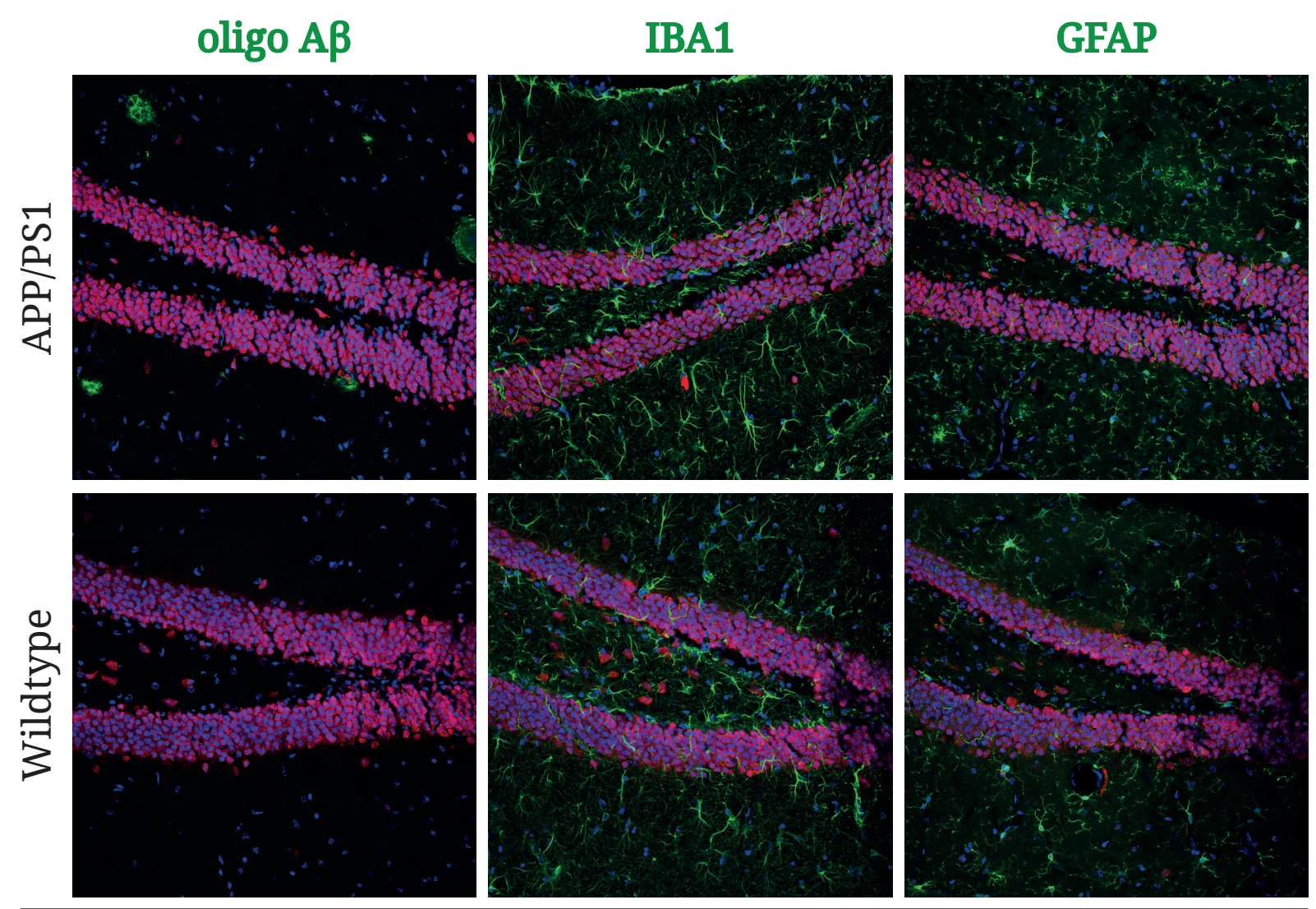

\section{DAPI}

\section{NeuN}

Figure 4.22 - Confocal imaging of the dentate gyrus from 4 months old mice:

Confocal imaging of amyloid plaques (left), microglia (center) and astrocytes (right) in brain slices from wildtype (top) and transgenic (bottom) mice using a magnification of 20x. Plaques, astrocytes (GFAP) and microglia (IBA1) are labeled in green, neurons (NeuN) in red and DAPI-stained nuclei are labeled in blue. Error bars resemble standard deviation. 


\subsection{Transcription factor analysis in APPPS1 mice}

One potential mechanism that might cause a deregulation of gene expression is a modification of TF activity. To identify potential TFs that might act as drivers for differential gene expression or that could be targeted to reinstate a healthy gene expression, I performed a TF binding site prediction analysis on the previously described RNAseq dataset using Pscan (Zambelli et al., 2009). A list of differentially expressed genes is parsed into Pscan which then screens the promoters for each gene and tries to align those to an implemented set of 263 TF binding sites, or motifs. A score is computed for each motif:promoter-pair and a p-value is given for each motif. For a more solid analysis, p-values were transformed into adjusted p-values. Transcription factors with an adjusted $p$-value $\leq 0.05$ and a motif length $\geq 8$ were considered as significant and scores $\geq 0.95$ were considered as likely true positive motif:promoter-pairs.

Due to the large overlap of differentially expressed genes in 4 and 8 months old transgenic mice, the majority of significant TFs found by Pscan is shared between the age groups (see figure 4.23A, p.84). A substantial number of highly significant TFs can be related to immune response, i.e. STAT1, SPI1, IRF1, or NFKB1 (see table 4.2, p.86). Thus, results from Pscan do nicely match data from functional pathway analysis. Figure 4.23B and C (see p.84) shows the coverage of differentially expressed genes targeted by significant TFs. A core-set of TFs was defined covering TF families known to function in neuronal plasticity and neural immune response, namely ETS, GATA, IRF, KLF, NF KB and STAT. A detailed list of TFs covered by Pscan and included in the coreset can be seen in table 7.17 (see p.136). 74-80\% of all differentially genes significant in 4 months old mice are likely to be regulated by at least one of the TFs included in the core-set consistently for all brain regions. The coverage only marginally changes in 8 months old transgenic mice. Only approximately 6 to $12 \%$ of genes expressed in either age group or brain region can not be linked to one of the TFs included in Pscan.

In the ACC and CA1, most of the genes that carried a motif belonging to the core-set of TFs were likely targeted by members of the ETS or KLF family. Only a very small fraction of genes might be regulated by proteins from the GATA family. The ETS is regulating a larger fraction of differentially expressed genes in the DG. Inside that TF family, the most significant hit is SPI1. Members from the KLF family covered by Pscan were restricted to KLF1, KLF4, and KLF5. Thus, some 
genes targeted by this family of TFs might not be identified by the described analysis yet.

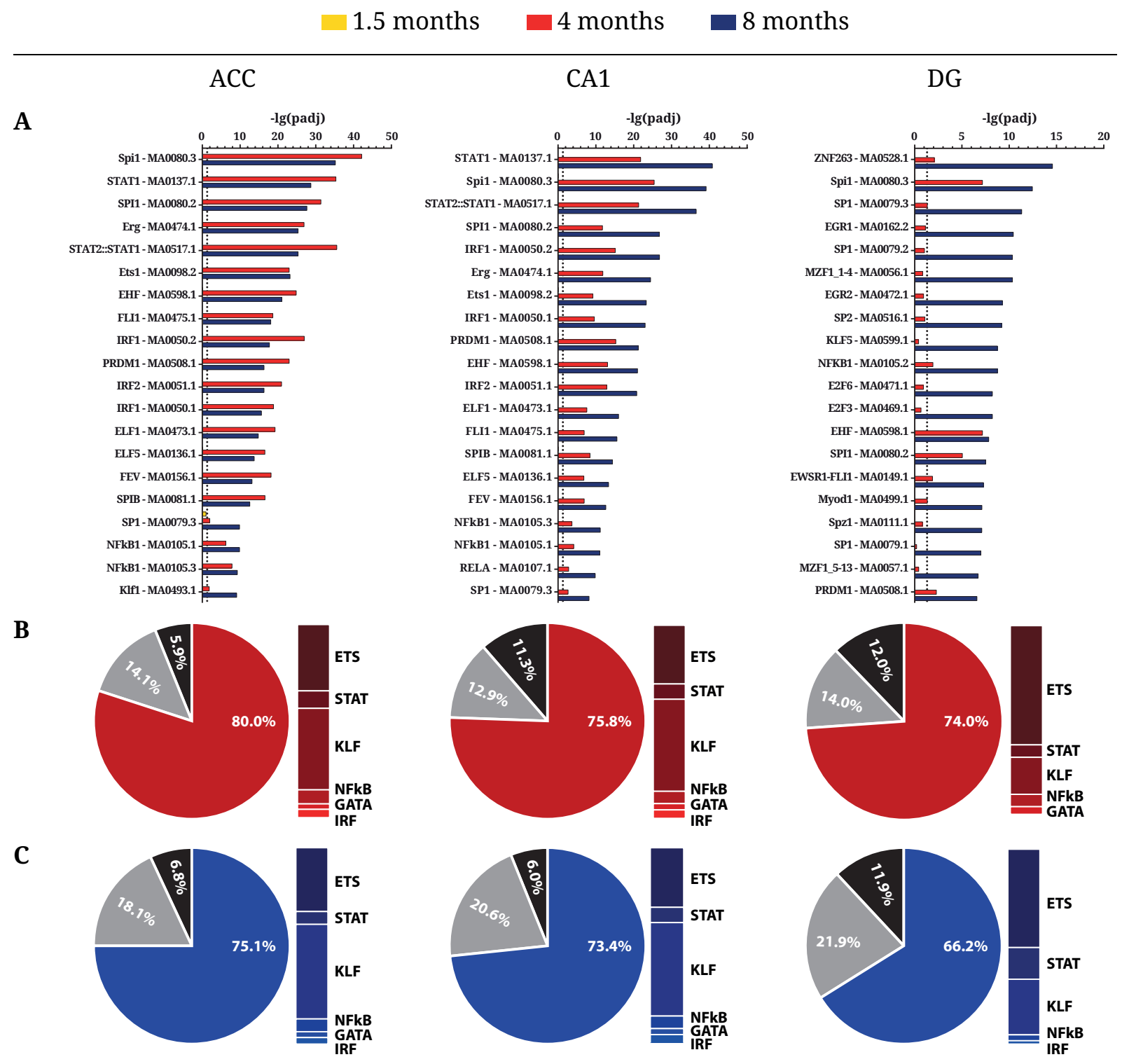

core-set targets

non-core-set targets

unassigned genes

Figure 4.23 - Overrepresented transcription factors in APP/PS1 transgenic mice:

A) Overrepresented TFs in 1.5 (yellow), 4 (red), and 8 (blue) months old APP/PS1 transgenic mice compared to wildtype mice identified by Pscan. The top 20 significant motifs identified for 8 months old mice were chosen for plotting. Dotted lines resemble the threshold for significance $(=0.05)$.

B/C) Pie-chart: Percentage of differentially expressed genes for 4 (top) and 8 (bottom) months old mice with motifs for either one of the core-set TFs, non-core-set TFs and those genes that do not carry any of the motifs included in the analysis (unassigned). Bar charts next to each pie chart resemble the relative coverage of target genes for each respective TF family included in the core-set. 
The activity of TFs depends on different parameters. Some TFs might form complexes with other proteins, some need to undergo post-translational modifications to become active. Another important parameter is the pure abundance of a respective TF which is mainly driven by gene expression itself. Thus, I checked for the level of gene expression from the core-set TFs in AP$\mathrm{P} / \mathrm{PS} 1$ transgenic mice. As shown in figure 4.24, only a small fraction of genes coding for core-set TFs is differentially expressed or mildly deregulated ( $\mid \log _{2}$ foldchange $\left.\mid \leq 0.5\right)$. Gene expression per se is stronger affected in 8 months old mice compared to 4 months old mice as previously shown in figure 4.5B (see p.63) and core-set TF coding genes are also following that pattern as the number of significant genes is higher in 8 months old mice. The core-set TFs affected in their gene expression are largely shared among the brain regions and included Irf1, Spi1 and Stat1 (all regions), Elf1, Fli1, Nfkb1, RelA, Stat3 and Stat6 (ACC and CA1), Klf4, and Stat4 (both exclusive for the ACC). The only significantly downregulated transcription factor in the given datasets is Stat4. All differentially expressed core-set TFs can be related with immune response. Additionally, $\mathrm{Nf}_{\mathrm{k}} \mathrm{B}$ and Stat3 were also implicated with neuronal signaling. Altogether, upregulation of the identified core-set TFs further induces immune response observed in APP/PS1 mice.

\section{significant: \& \&} mildly deregulated: \& \&

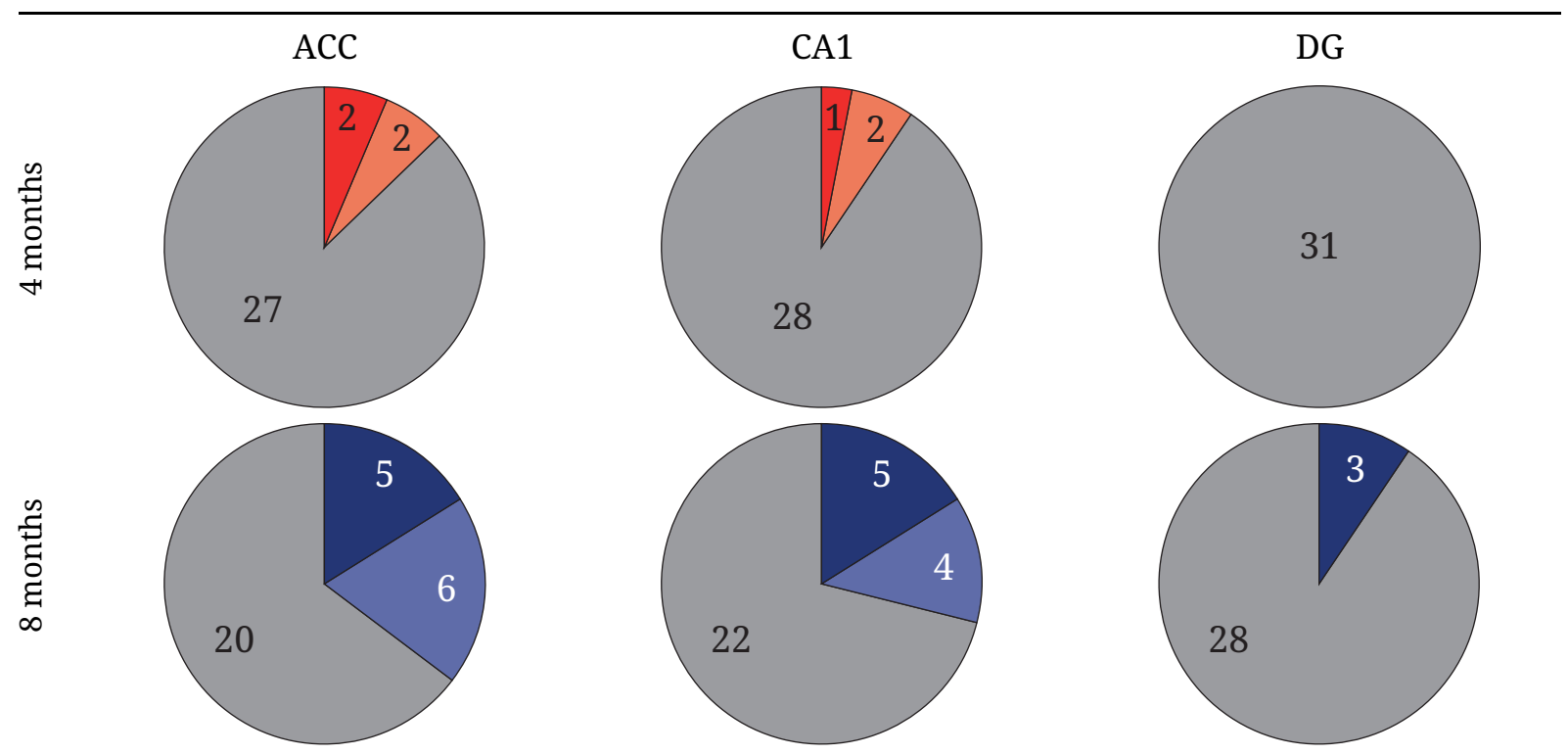

Figure 4.24 - Differentially expressed transcription factors in APP/PS1 mice:

Numbers of differentially expressed and mildly deregulated genes corresponding to core-set TFs in 4 (red, top) and 8 (blue, bottom) months old APP/PS1 mice. Transcription factor coding genes not affected in their gene expression are shown in grey. 
Table 4.2 - Biological function of deregulated Transcription factors

\begin{tabular}{|c|c|c|}
\hline TF & effect & reference \\
\hline Elf1 & induction of immune response & $\begin{array}{l}\text { Sharrocks (2001); Wang et al. } \\
\text { (1993a) }\end{array}$ \\
\hline FLI1 & TGF- $\beta$ signaling; tumor suppression & $\begin{array}{l}\text { Hahm et al. (1999); Sharrocks } \\
(2001)\end{array}$ \\
\hline IRF1 & activation of immune response, tumor suppression & $\begin{array}{l}\text { Miyamoto et al. (1988); Xie et al. } \\
\text { (2003) }\end{array}$ \\
\hline KLF4 & induction of apoptosis, tumor suppression & $\begin{array}{l}\text { (El-Karim et al., 2013; Rowland } \\
\text { et al., 2005) }\end{array}$ \\
\hline $\mathrm{NF} \kappa \mathrm{B}$ & $\begin{array}{l}\text { proliferation, cell survival, neuronal plasticity, induction } \\
\text { of immune response }\end{array}$ & $\begin{array}{l}\text { Brantley et al. (2001); Hayden } \\
\text { et al. (2006); Mattson and Caman- } \\
\text { dola (2001) }\end{array}$ \\
\hline RELA & NF $k B$ subunit p65 & see $N_{F} \kappa B$ \\
\hline SPI1 & differentiation of microglia & $\begin{array}{l}\text { Sharrocks (2001); Suzuki et al. } \\
\text { (2003) }\end{array}$ \\
\hline STAT1 & induction of immune response and apoptosis & $\begin{array}{l}\text { Frank et al. (1999); Takagi et al. } \\
\text { (2002) }\end{array}$ \\
\hline STAT3 & neuronal signaling, immune suppression & Chiba et al. (2009); Yu et al. (2007) \\
\hline STAT4 & induction of immune response & $\begin{array}{l}\text { (Deng et al., 2004; Wurster et al., } \\
\text { 2000) }\end{array}$ \\
\hline STAT6 & induction of immune response & $\begin{array}{l}\text { (Goenka and Kaplan, 2011; } \\
\text { Wurster et al., 2000) }\end{array}$ \\
\hline
\end{tabular}

\subsection{Differential splicing in APPPS1 transgenic mice}

Differential gene expression analysis and corresponding transcriptin factor binding site prediction indicate severe changes on the transcription machinery following amyloidosis. This disruption might not only resemble changes in transcription frequency but also differential exon usage. Thus, I studied alternate splicing based on RNAseq datasets from 1.5, 4, and 8 months old mice using the R package DEXSeq (Anders et al., 2012). DEXSeq is able to detect relatively mild changes in exon usage, thus a $\log _{2}$ foldchange cutoff of $\pm 0.15(\approx 10 \%)$ was used for the whole analysis.

Figure 4.25A (see p.87) shows volcano plots for significance and fold change of differentially expressed exons. Only few exons were differentially expressed in all three brain regions and age groups with the exception of the ACC of 1.5 months old mice, where a significant number 


\section{5 months $\quad 4$ months $\quad 8$ months}

A

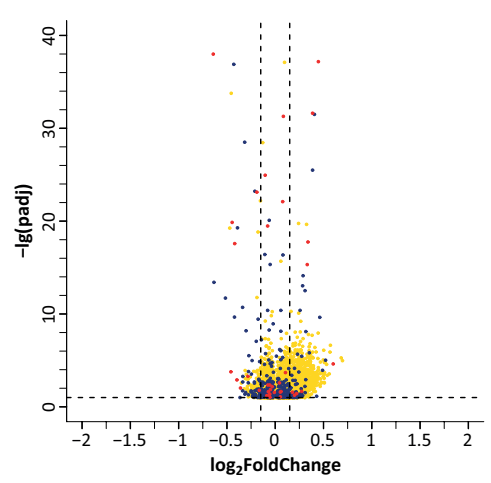

B

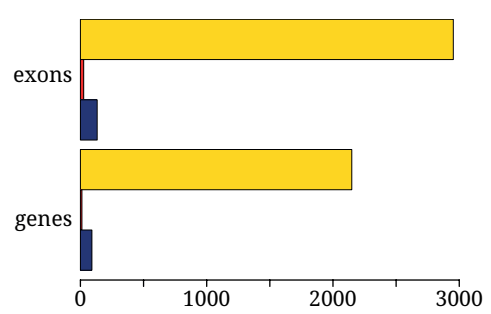

C

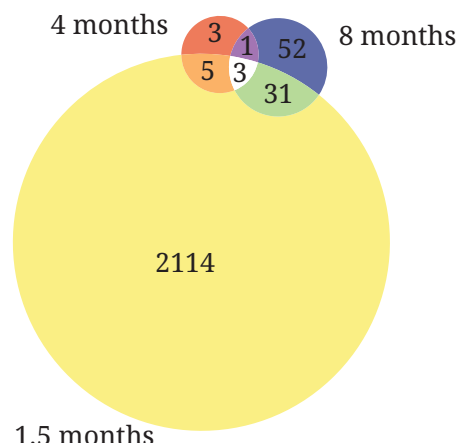

CA1

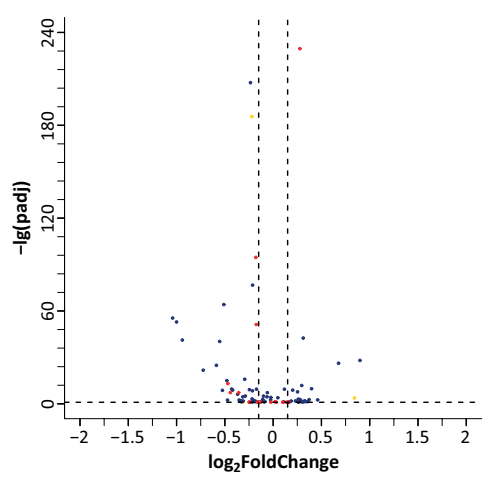

log $_{2}$ FoldChange
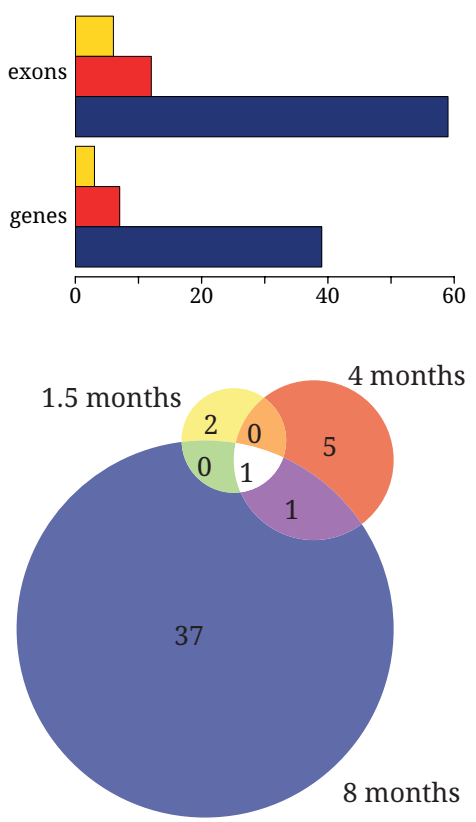

DG
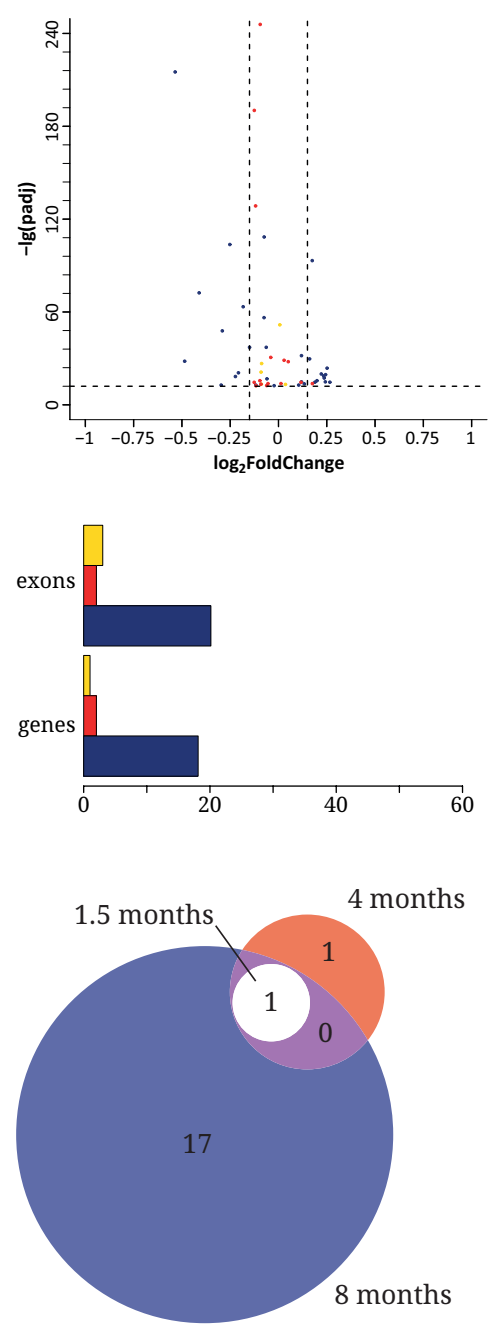

Figure 4.25 - Differiental splicing in APP/PS1 transgenic mice:

A) Volcano plots for exons detected by the DEXSeq analysis for 1.5 (yellow), 4 (red) and 8 (blue) months old transgenic mice. Only exons with an adjusted $p$-value $\leq 0.1$ are plotted for technical reasons. Dashed lines resemble the threshold for significance of 0.1 (horizontal) and the cutoffs for $\log _{2}$ foldchange of \pm 0.15 made for analysis.

B) Number of differentially spliced exons and corresponding number of differentially spliced genes. Note the different scaling for $y$-axis in the ACC.

C) Venn diagrams showing the number of differentially spliced genes common or exclusive for the three age groups.

of exons was upregulated in transgenic mice compared to wildtype littermates. The number of differentially spliced genes in each respective brain region and age group is shown in figure 4.25 (B). Approximately 1.4 exons per differentially spliced gene were significant in the ACC of 1.5 
months old mice. Except for the ACC of young mice, the differential expression profile of exons is similar to gene expression, as the number of differentially spliced genes is increasing during aging. However, the number of deregulated exons is only marginal compared to those from gene expression (see figure 4.5, p.63). Only few genes are differentially spliced in all age groups and tested brain regions (see figure 4.25C, p.87). These genes include APP cleaving enzymes like PS1 and CTSD, the APP recognizing enzyme TREM2 and APP itself.

Table 4.3 - Overrepresented KEGG-pathways for differential splicing in young APP/PS1 mice

\begin{tabular}{|c|c|c|c|}
\hline KEGG pathway & Represented genes & p-value & adjusted p-value \\
\hline Long-term potentiation & 25 & $2.16 \mathrm{E}-07$ & 3.80E-05 \\
\hline Huntington's disease & 42 & 7.01E-06 & 6.17E-04 \\
\hline Lysosome & 31 & 1.09E-05 & $6.40 \mathrm{E}-04$ \\
\hline Alzheimer's disease & 39 & 7.98E-05 & $3.50 \mathrm{E}-03$ \\
\hline Regulation of actin cytoskeleton & 44 & $9.94 \mathrm{E}-05$ & $3.49 \mathrm{E}-03$ \\
\hline Parkinson's disease & 31 & 1.05E-04 & 3.09E-03 \\
\hline Neurotrophin signaling pathway & 30 & $1.68 \mathrm{E}-04$ & 4.23E-03 \\
\hline Citrate cycle (TCA cycle) & 12 & $3.17 \mathrm{E}-04$ & $6.95 \mathrm{E}-03$ \\
\hline MAPK signaling pathway & 49 & $3.82 \mathrm{E}-04$ & $7.45 \mathrm{E}-03$ \\
\hline Oxidative phosphorylation & 29 & 4.03E-04 & 7.07E-03 \\
\hline Oocyte meiosis & 26 & 7.03E-04 & $1.12 \mathrm{E}-02$ \\
\hline Endocytosis & 39 & 7.33E-04 & $1.07 \mathrm{E}-02$ \\
\hline GnRH signaling pathway & 23 & $7.95 \mathrm{E}-04$ & $1.07 \mathrm{E}-02$ \\
\hline Calcium signaling pathway & 37 & $9.68 \mathrm{E}-04$ & $1.21 \mathrm{E}-02$ \\
\hline Spliceosome & 27 & $9.83 \mathrm{E}-04$ & $1.15 \mathrm{E}-02$ \\
\hline Fc gamma R-mediated phagocytosis & 22 & $2.22 \mathrm{E}-03$ & $2.41 \mathrm{E}-02$ \\
\hline Glioma & 16 & $3.75 \mathrm{E}-03$ & $3.82 \mathrm{E}-02$ \\
\hline Ubiquitin mediated proteolysis & 27 & $3.88 \mathrm{E}-03$ & $3.73 \mathrm{E}-02$ \\
\hline Alanine, aspartate and glutamate metabolism & 10 & 4.16E-03 & 3.79E-02 \\
\hline
\end{tabular}

Since the quality of RNAseq was validated for each sample and data from differential gene expression, the exceptional differential splicing in the ACC of 1.5 months old mice is unlikely to be an artifact of sequencing or an unspecific output from DEXSeq. To get further insight into these data, I screened the differentially spliced genes for overrepresented functional pathways (KEGG pathways). Nineteen significant KEGG pathways with an adjusted $p$-value $\leq 0.05$ were identified 
that can mainly be categorized in neuronal plasticity ("Long-term Potentiation", "Calcium Signaling Pathway"), neurodegenerative diseases ("Alzheimer’s Disease”, ”Parkinson’s disease”) or metabolism ("Citrate Cycle", "Oxidative Phosphorylation"), whereby a large proportion of genes is shared among pathways of a similar category (see table 4.3, p.88).

\subsection{Differential splicing during aging}

\subsubsection{Differential splicing in healthy aging}

The relatively low abundance of differential splicing upon amyloidosis was unexpected an led to the question if splicing is also just marginally affected during aging. I thus performed a similar analysis on aging wildtype and transgenic mice. Figure 4.26A (see p.90) shows the adjusted pvalue and $\log _{2}$ foldchange of exons for each age comparison in wildtype mice's ACC, CA1 and DG. For computational and visual purposes, exons with an adjusted p-value below 0.1 are not shown and the y-dimension of each plot is designed to show the bottom $99.9 \%$ of significant data. The majority of significantly differentially transcribed exons is only mildly affected $(<10 \%)$. While the changes in splicing seem to be negligible in the CA1, a number of differentially transcribed exons can be found in the other two regions. The most differences do hereby occur when comparing 4 and 8 months old mice. Gene expression in healthy wildtype mice revealed a mild tendency towards downregulation in the ACC and a rather neutral ratio in the DG (see figure 4.10, p.70). In contrast, the majority of exons in the ACC of both aging comparisons is upregulated suggesting a trend towards inclusion of exons, while a slight tendency towards exon exclusion can be observed in the DG of 4 vs 8 months old mice.

A minimum of 590 exons corresponding to 414 genes (DG in early-life aging) are found to change with age regarding the ACC and DG. The most significant differences can though be found in the ACC of 4 vs 8 months old mice (15,891 exons from 4699) (see figure 4.26B, p.90). While approximately 3.4 exons per gene were significantly differentially transcribed in the ACC of midlife aging mice, the majority of genes in the other comparisons contained only a single significant exon ( $\hat{x}$ : 1.2 - 1.4). In the ACC, 524 out of all 689 differentially spliced genes in early-life aging were also significant in the mid-life aging. $78 \%$ of the commonly significant exons change in 


\section{5 vs 4 months 4 vs 8 months}

ACC

A

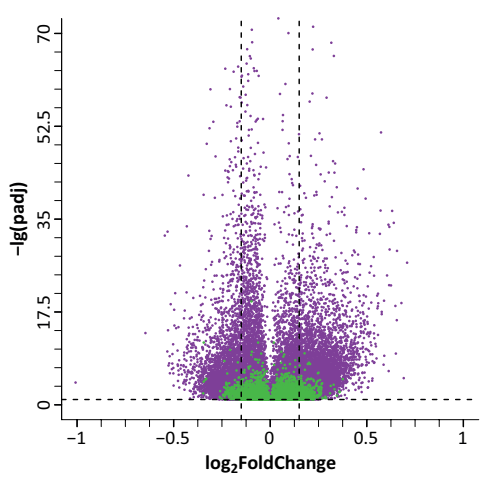

B

C
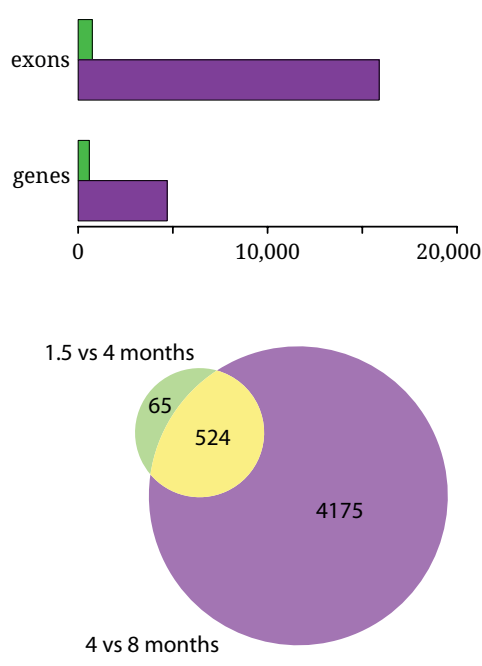

CA1
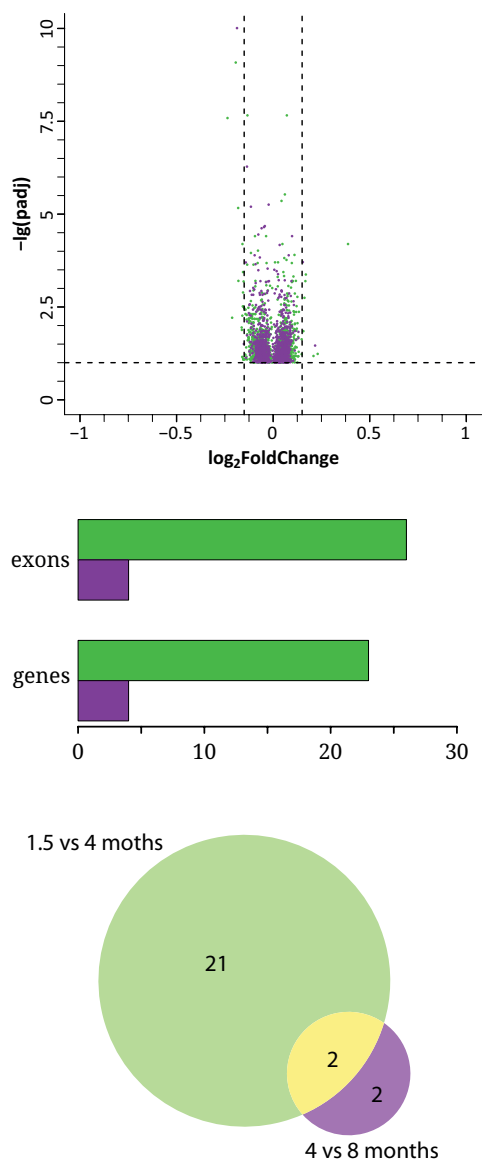

DG
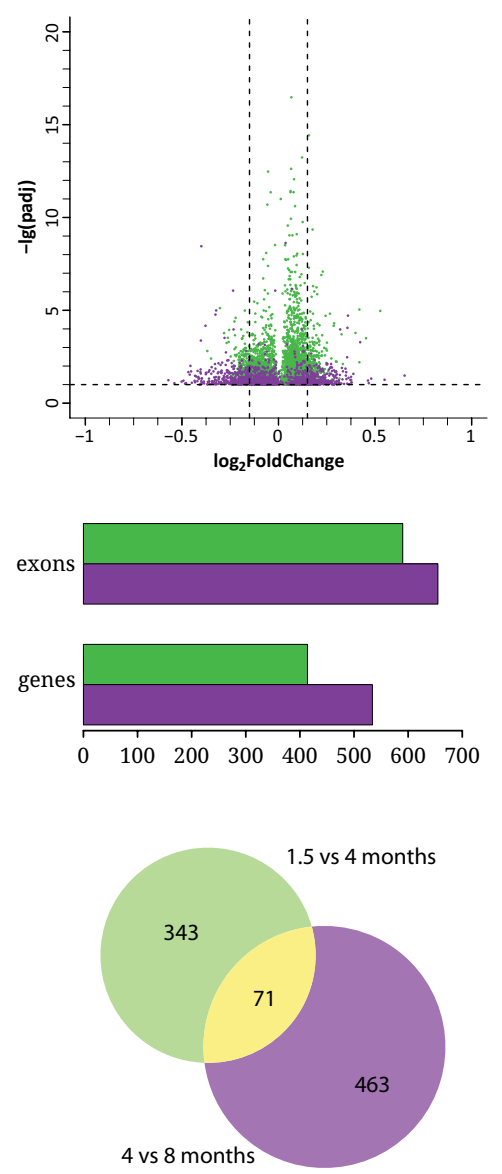

Figure 4.26 - Differiental splicing in aging wildtype mice:

A) Volcano plots for exons detected by the DEXSeq analysis for 1.5 vs 4 (green) and 4 vs 8 (purple) months old transgenic mice. Only exons with an adjusted p-value $\leq 0.1$ are plotted for technical reasons. Dashed lines resemble the threshold for significance of 0.1 (horizontal) and the cutoffs for $\log _{2}$ foldchange of \pm 0.15 made for analysis.

B) Number of differentially spliced exons and corresponding number of differentially spliced genes. Note the different scaling for $y$-axis.

C) Venn diagrams showing the number of differentially spliced genes common or exclusive for the three age groups.

the same direction throughout aging. In contrast, from all 71 common exons in the DG, 23 are developing in opposite directions throughout aging.

To check whether differential splicing affects certain functional pathways in aging mice, I screened for overrepresented KEGG pathways. Only a single significant pathway (adjusted pvalue $\leq 0.05$ ) was found for the ACC and DG of 1.5 vs 4 months old mice, namely "Spliceosome" 
and "Tight Junction" respectively (see table 4.4, p.92). The "Spliceosome"-pathway is also significantly represented in 4 vs 8 months old mice (both ACC and DG). Due to the high number of differentially spliced genes, the most significant pathways appear in the ACC of 4 vs 8 months old mice and those pathways cover a broad range of biological functions mainly involved in neuronal signaling ("Axonal guidance", "Long-Term Potentiation"), general signal transduction ("Phosphatidylinositol signaling system") and gene expression ("Aminoacyl-rRNA biosynthesis", "Spliceosome") (see table 4.4, p.92). No significant pathways were found for differentially spliced genes that are inversely changing during aging.

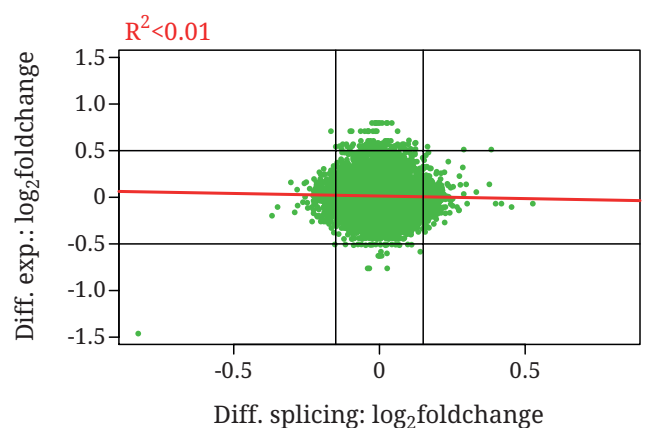

C

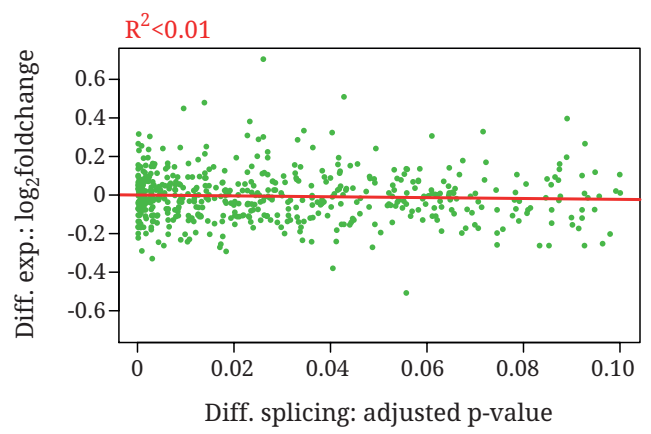

B

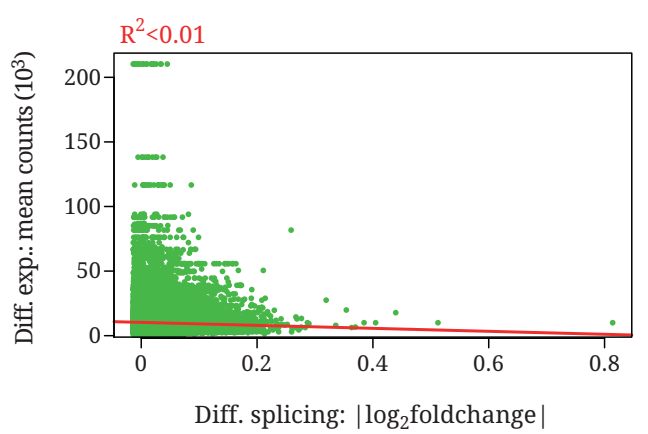

D

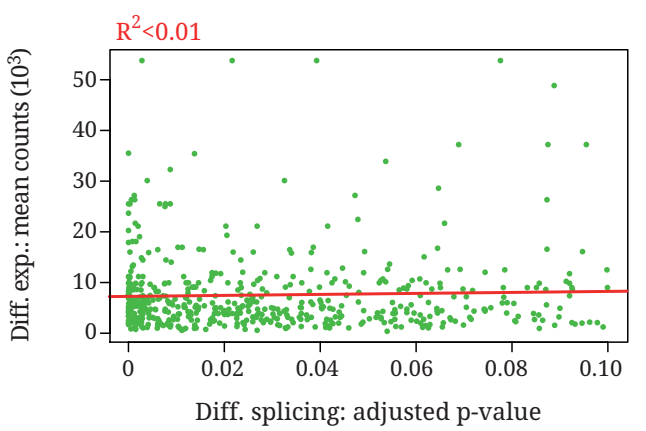

Figure 4.27 - Comparison of differential gene expression and splicing analysis:

A linear regression for each comparison is displayed in red and the $\mathrm{R}^{2}$ value for each correlation analysis is written ontop of each plot.

A) Comparison of the $\log _{2}$ foldchanges from each respective analysis. Horizontal lines resemble the cutoff for gene expression analysis and vertical lines cutoff for differential splicing. Note that in the given example differential gene expression and splicing are almost mutually exclusive. No correlation can be found between the foldchanges.

B) Normalized mean counts from gene expression analysis versus absolute $\log _{2}$ foldchange for splicing. No correlation can be found between the two given parameters. Differential splicing seems to be less likely in highest expressed genes.

C) $\log _{2}$ foldchange from gene expression plotted against the adjusted p-value from splicing analysis (with padj $\leq 0.05)$. No correlation can be found here as well.

D) Comparison of the normalized read counts from gene expression and the adjusted p-value from splicing analysis. Only values for significant exons were plotted. Significance of differentially transcribed exons appears independent from read count of the corresponding gene. 
Due to the high number of significant exons and the resulting broad range of functional pathways, I investigated whether the data is contaminated with too many false positives. Since quality control of the RNAseq data confirmed a high quality, I hypothesized that any significant fraction of false positives could either derive from a very high basal gene expression or differential gene expression. I thus checked whether one can correlate either read counts or foldchanges from differential gene expression analysis with the exon foldchange and adjusted p-value (see figure 4.27, p.91). As shown in 4.27A (see p.91), differential gene expression and alternative splicing seem to be mutually exclusive in the given example (DG of 1.5 vs 4 months old wildtype mice). Only exons from genes that had an absolute $\log _{2}$ foldchange below 0.5 were significant in splicing analysis, as it is also shown in 4.27C (see p.91). Also, no correlation could be found between the exons' foldchanges and basal gene expression of corresponding genes which is described by the normalized read count from gene expression analysis. However, it seems that the highest expressed genes are more unlikely to be differentially spliced. Taken together, these data indicate that there is no correlation between gene expression and differential splicing. Any potential artificial effects that might cause a high number of false positive values are thus unlikely a result from the data or the chosen cutoffs for analysis but rather a limitation of the utilized DEXSeq package.

Table 4.4 - Overrepresented KEGG pathways for differential splicing in aging wildtype mice

\begin{tabular}{|c|c|c|}
\hline $\begin{array}{l}1.5 \text { vs } 4 \text { months: ACC } \\
\text { pathway }\end{array}$ & adj. p-value & genes \\
\hline Spliceosome & 0.0023 & $\begin{array}{l}\text { PRPF31, RBM25, DDX5, SRSF5, DDX46, HNRNPK, } \\
\text { PRPF40B, TCERG1, SF3B2, SF3B1, PRPF38B, WBP11, } \\
\text { U2AF2, SRSF2, HNRNPU }\end{array}$ \\
\hline $\begin{array}{l}1.5 \text { vs } 4 \text { months: DG } \\
\text { pathway }\end{array}$ & adj. p-value & genes \\
\hline Tight junction & 0.0427 & $\begin{array}{l}\text { AKT2, CDK4, ACTN1, AKT3, SPTBN1, SYMPK, } \\
\text { EPB4.1L3, MPDZ, CTTN, PRKCB, ACTN4, MLLT4 }\end{array}$ \\
\hline $\begin{array}{l}4 \text { vs } 8 \text { months: DG } \\
\text { way }\end{array}$ & adj. p-value & genes \\
\hline Spliceosome & 0.0022 & $\begin{array}{l}\text { DDX23, SRSF6, SRSF1, XAB2, DDX5, PRPF8, THOC1, } \\
\text { SF3B1, SRSF2, DHX38, SART1, AQR, CHERP, HNRNPM }\end{array}$ \\
\hline Ubiquitin mediated proteolysis & 0.0124 & $\begin{array}{l}\text { UBA1, PPIL2, NEDD4L, HUWE1, TRIP12, PIAS3, } \\
\text { HERC3, NEDD4, UBE2Q2, UBA7, CDC16, HERC1, } \\
\text { UBE3C, GM20661 }\end{array}$ \\
\hline
\end{tabular}


Table 4.5 - KEGG pathways for differential splicing in wildtype ACC: 4 vs 8 months (1)

\begin{tabular}{|c|c|c|}
\hline KEGG pathway & genes & ad. p-value \\
\hline Axon guidance & 65 & 3.01E-08 \\
\hline Long-term potentiation & 42 & 2.75E-08 \\
\hline Endocytosis & 88 & $2.34 \mathrm{E}-08$ \\
\hline Ubiquitin mediated proteolysis & 66 & 1.77E-08 \\
\hline ErbB signaling pathway & 47 & 9.43E-08 \\
\hline Phosphatidylinositol signaling system & 42 & $1.60 \mathrm{E}-07$ \\
\hline Spliceosome & 59 & $2.50 \mathrm{E}-07$ \\
\hline mTOR signaling pathway & 33 & $4.28 \mathrm{E}-07$ \\
\hline Regulation of actin cytoskeleton & 86 & $2.66 \mathrm{E}-06$ \\
\hline Insulin signaling pathway & 61 & $2.68 \mathrm{E}-06$ \\
\hline MAPK signaling pathway & 99 & $6.24 \mathrm{E}-06$ \\
\hline Focal adhesion & 78 & $1.11 \mathrm{E}-05$ \\
\hline Neurotrophin signaling pathway & 54 & $1.15 \mathrm{E}-04$ \\
\hline Glioma & 32 & $1.50 \mathrm{E}-04$ \\
\hline Aminoacyl-tRNA biosynthesis & 24 & $1.50 \mathrm{E}-04$ \\
\hline Renal cell carcinoma & 34 & $1.43 \mathrm{E}-04$ \\
\hline Fc gamma R-mediated phagocytosis & 43 & $1.82 \mathrm{E}-04$ \\
\hline Inositol phosphate metabolism & 28 & $2.07 \mathrm{E}-04$ \\
\hline Lysine degradation & 23 & $2.81 \mathrm{E}-04$ \\
\hline Lysosome & 49 & $2.78 \mathrm{E}-04$ \\
\hline Progesterone-mediated oocyte maturation & 38 & $3.08 \mathrm{E}-04$ \\
\hline Adherens junction & 35 & $3.06 \mathrm{E}-04$ \\
\hline Circadian rhythm & 11 & $5.84 \mathrm{E}-04$ \\
\hline GnRH signaling pathway & 41 & $5.88 \mathrm{E}-04$ \\
\hline Chronic myeloid leukemia & 34 & $7.21 \mathrm{E}-04$ \\
\hline Adipocytokine signaling pathway & 31 & $7.22 \mathrm{E}-04$ \\
\hline Glycerophospholipid metabolism & 31 & 7.22E-04 \\
\hline Prostate cancer & 38 & $1.04 \mathrm{E}-03$ \\
\hline RNA degradation & 28 & 1.33E-03 \\
\hline Pathways in cancer & 104 & $1.80 \mathrm{E}-03$ \\
\hline Endometrial cancer & 25 & $1.74 \mathrm{E}-03$ \\
\hline Wnt signaling pathway & 55 & $1.75 \mathrm{E}-03$ \\
\hline
\end{tabular}


Table 4.6 - KEGG pathways for differential splicing in wildtype ACC: 4 vs 8 months (2)

\begin{tabular}{|l|c|c|}
\hline KEGG pathway & genes & ad. p-value \\
\hline Colorectal cancer & 35 & $3.79 \mathrm{E}-03$ \\
\hline Cardiac muscle contraction & 32 & $5.64 \mathrm{E}-03$ \\
\hline Pancreatic cancer & 30 & $6.14 \mathrm{E}-03$ \\
\hline $\begin{array}{l}\text { Arrhythmogenic right ventricular cardiomy- } \\
\text { opathy (ARVC) }\end{array}$ & 30 & $1.25 \mathrm{E}-02$ \\
\hline Acute myeloid leukemia & 24 & $1.77 \mathrm{E}-02$ \\
\hline Non-small cell lung cancer & 23 & $1.82 \mathrm{E}-02$ \\
\hline Calcium signaling pathway & 62 & $2.22 \mathrm{E}-02$ \\
\hline Long-term depression & 28 & $2.55 \mathrm{E}-02$ \\
\hline Valine, leucine and isoleucine degradation & 20 & $2.58 \mathrm{E}-02$ \\
\hline Tight junction & 46 & $2.65 \mathrm{E}-02$ \\
\hline Oocyte meiosis & 40 & $3.18 \mathrm{E}-02$ \\
\hline SNARE interactions in vesicular transport & 17 & $3.67 \mathrm{E}-02$ \\
\hline Dilated cardiomyopathy & 33 & $3.96 \mathrm{E}-02$ \\
\hline Fc epsilon RI signaling pathway & 30 & $4.17 \mathrm{E}-02$ \\
\hline Gap junction & 31 & $4.49 \mathrm{E}-02$ \\
\hline T cell receptor signaling pathway & 40 & $4.50 \mathrm{E}-02$ \\
\hline Limonene and pinene degradation & 28 & $4.56 \mathrm{E}-02$ \\
\hline VEGF signaling pathway & $4.54 \mathrm{E}-02$ \\
\hline Type II diabetes mellitus & $4.48 \mathrm{E}-02$ \\
\hline
\end{tabular}

\subsubsection{Differential splicing in aging transgenic mice}

The differences found between wildtype and transgenic mice regarding splicing were relatively little, except from differences in the ACC of 1.5 months old mice (see figure 4.25A, p.87). However, quite some differences were found throughout aging in wildtype mice. To confirm the data from wildtype-vs-transgenic comparisons and to shed some light on splicing in aging transgenic mice, I did a similar analysis as described above for transgenic mice. By comparing the volcano plots from wildtype aging (see figure 4.26A, p.90) and transgenic aging (see figure 4.28A, p.95), one can identify certain similarities between the two sets of data. As it was observed for wildtype mice, a strong effect of mid-life aging on splicing can be found in the ACC, while the effect on the CA1 in both aging comparisons is rather negligible. Different from wildtype mice, there are only very 


\section{5 vs 4 months 4 vs 8 months}

A

ACC

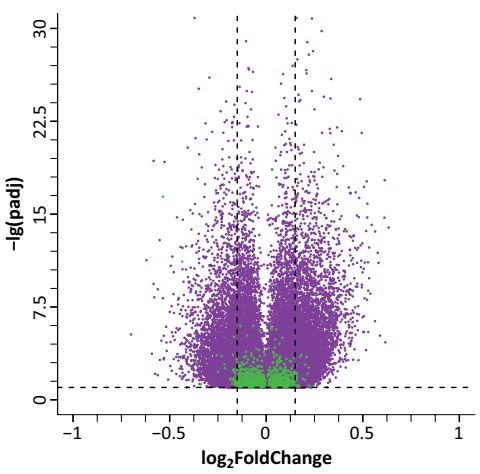

B

C
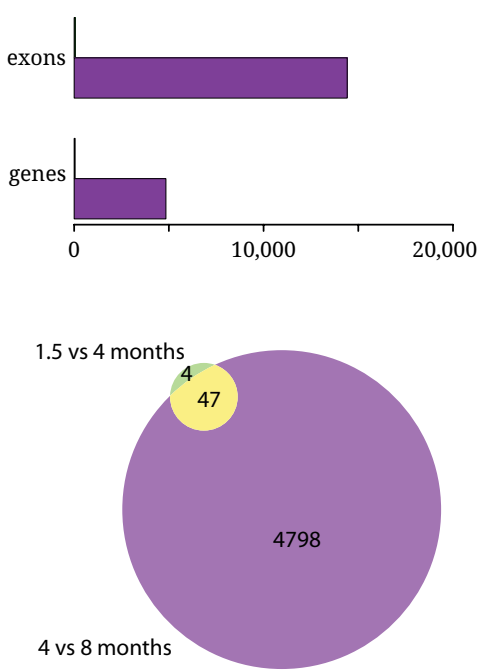

CA1
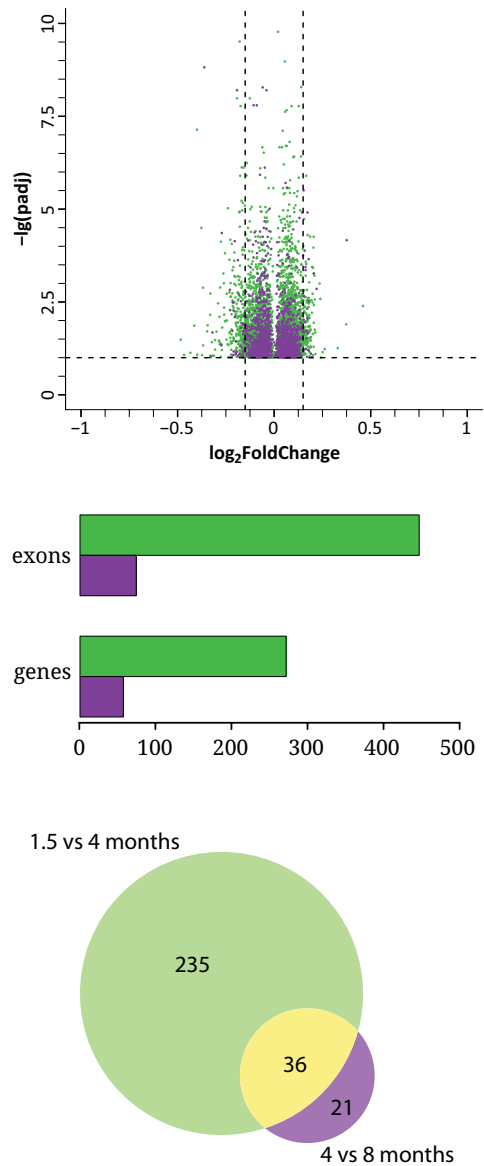

DG
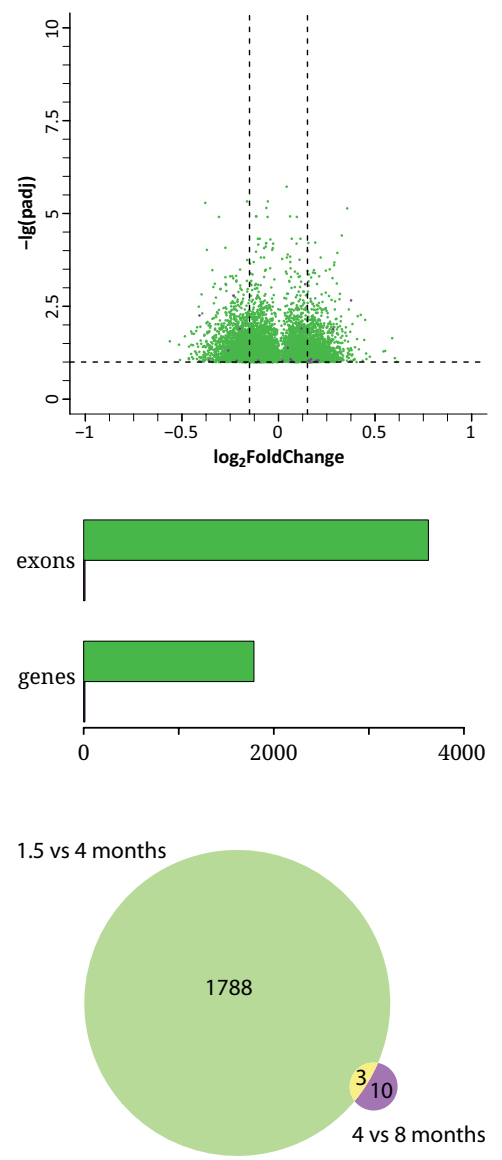

Figure 4.28 - Differiental splicing in aging transgenic mice:

A) Volcano plots for exons detected by the DEXSeq analysis for 1.5 vs 4 (green) and 4 vs 8 (purple) months old transgenic mice. Only exons with an adjusted p-value $\leq 0.1$ are plotted for technical reasons. Dashed lines resemble the threshold for significance of 0.1 (horizontal) and the cutoffs for $\log _{2}$ foldchange of \pm 0.15 made for analysis.

B) Number of differentially spliced exons and corresponding number of differentially spliced genes. Note the different scaling for y-axis.

C) Venn diagrams showing the number of differentially expressed exons common and age exclusive for the three age groups.

few exons differentially transcribed in the ACC of 1.5 vs 4 months old and the DG of 4 vs 8 months old transgenic mice. 4845 genes were differentially spliced in the ACC during mid-life aging and each gene showed in average 3 affected exons (see figure 4.28B). In the DG of 1.5 vs 4 months old mice where 1791 genes were differentially expressed, each gene resembles in average 2 exons. From the few genes differentially spliced in the ACC during early-life aging, the majority is also 
significant later on (see figure 4.28C, p.95).

Pathway analysis from alternative splicing in wildtype mice revealed a broad spectrum with particular enrichment of neuronal function related pathways and signal transduction. The majority of pathways identified for the ACC of mid-life aging transgenic mice was found to be similar in comparison to wildtype mice (see table 4.8, p.97). Notably, one of the 8 pathways unique for transgenic mice is "Alzheimer's Disease". With only one exception, all pathways overrepresented in the DG of early-life aging transgenic mice are also significant in the ACC during mid life aging. The pathway "Alzheimer's Disease", however, is not among the significant ones in the DG. Though some differential splicing can be linked with amyloid pathology, these data from wildtype and transgenic mice suggest that differential splicing is a crucial mechanism for aging and is only mildly affected by the transgenic induction of APP and PS1.

Table 4.7 - Overrepresented KEGG pathways for differential splicing in early-life aging transgenic mice: DG

\begin{tabular}{|l|c|c|}
\hline KEGG pathway & genes & ad. p-value \\
\hline Ubiquitin mediated proteolysis & 35 & $8.97 \mathrm{E}-06$ \\
\hline Phosphatidylinositol signaling system & 24 & $1.30 \mathrm{E}-05$ \\
\hline Spliceosome & 31 & $3.97 \mathrm{E}-05$ \\
\hline MAPK signaling pathway & 47 & $8.80 \mathrm{E}-04$ \\
\hline Endocytosis & 37 & $3.25 \mathrm{E}-03$ \\
\hline GnRH signaling pathway & 22 & $5.25 \mathrm{E}-03$ \\
\hline Gap junction & 20 & $6.85 \mathrm{E}-03$ \\
\hline Inositol phosphate metabolism & 15 & $6.66 \mathrm{E}-03$ \\
\hline Focal adhesion & 33 & $2.53 \mathrm{E}-02$ \\
\hline Insulin signaling pathway & 25 & $3.25 \mathrm{E}-02$ \\
\hline ErbB signaling pathway & 18 & $3.73 \mathrm{E}-02$ \\
\hline RNA degradation & 14 & $4.20 \mathrm{E}-02$ \\
\hline $\begin{array}{l}\text { Arrhythmogenic right ventricular cardiomy- } \\
\text { opathy (ARVC) }\end{array}$ & 16 & $4.39 \mathrm{E}-02$ \\
\hline
\end{tabular}


Table 4.8 - KEGG pathways for differential splicing in mid-life aging transgenic mice: ACC (1)

\begin{tabular}{|c|c|c|}
\hline KEGG pathway & genes & ad. p-value \\
\hline Phosphatidylinositol signaling system & 45 & 3.19E-08 \\
\hline Insulin signaling pathway & 67 & 9.45E-08 \\
\hline ErbB signaling pathway & 48 & 1.16E-07 \\
\hline Renal cell carcinoma & 41 & $1.60 \mathrm{E}-07$ \\
\hline Axon guidance & 63 & $2.06 \mathrm{E}-07$ \\
\hline mTOR signaling pathway & 34 & $2.52 \mathrm{E}-07$ \\
\hline Glioma & 38 & $2.31 \mathrm{E}-07$ \\
\hline Endocytosis & 86 & $2.55 \mathrm{E}-07$ \\
\hline Focal adhesion & 83 & $9.81 \mathrm{E}-07$ \\
\hline Long-term potentiation & 38 & $3.96 \mathrm{E}-06$ \\
\hline Lysosome & 55 & $5.24 \mathrm{E}-06$ \\
\hline MAPK signaling pathway & 101 & $6.34 \mathrm{E}-06$ \\
\hline Regulation of actin cytoskeleton & 86 & $7.25 \mathrm{E}-06$ \\
\hline Neurotrophin signaling pathway & 58 & $8.00 \mathrm{E}-06$ \\
\hline Ubiquitin mediated proteolysis & 59 & $1.81 \mathrm{E}-05$ \\
\hline Spliceosome & 55 & $1.80 \mathrm{E}-05$ \\
\hline Colorectal cancer & 40 & $1.52 \mathrm{E}-04$ \\
\hline Endometrial cancer & 28 & $1.51 \mathrm{E}-04$ \\
\hline Alanine, aspartate and glutamate metabolism & 19 & $3.08 \mathrm{E}-04$ \\
\hline Inositol phosphate metabolism & 28 & 3.29E-04 \\
\hline Fc gamma R-mediated phagocytosis & 43 & $3.16 \mathrm{E}-04$ \\
\hline RNA degradation & 30 & 3.49E-04 \\
\hline Prostate cancer & 40 & $4.04 \mathrm{E}-04$ \\
\hline Progesterone-mediated oocyte maturation & 38 & $5.34 \mathrm{E}-04$ \\
\hline Adherens junction & 35 & $5.14 \mathrm{E}-04$ \\
\hline Lysine degradation & 22 & $1.21 \mathrm{E}-03$ \\
\hline Chronic myeloid leukemia & 34 & $1.23 \mathrm{E}-03$ \\
\hline Non-small cell lung cancer & 26 & $2.16 \mathrm{E}-03$ \\
\hline Acute myeloid leukemia & 27 & $2.14 \mathrm{E}-03$ \\
\hline Pancreatic cancer & 32 & $2.07 \mathrm{E}-03$ \\
\hline Pathways in cancer & 106 & 2.13E-03 \\
\hline Calcium signaling pathway & 66 & $7.55 \mathrm{E}-03$ \\
\hline GnRH signaling pathway & 38 & 8.64E-03 \\
\hline ABC transporters & 21 & $1.28 \mathrm{E}-02$ \\
\hline
\end{tabular}


Table 4.9 - KEGG pathways for differential splicing in mid-life aging transgenic mice: ACC (2)

\begin{tabular}{|l|c|c|}
\hline KEGG pathway & genes & ad. p-value \\
\hline Gap junction & 34 & $1.28 \mathrm{E}-02$ \\
\hline Adipocytokine signaling pathway & 28 & $1.33 \mathrm{E}-02$ \\
\hline T cell receptor signaling pathway & 43 & $1.79 \mathrm{E}-02$ \\
\hline Fatty acid elongation in mitochondria & 7 & $1.76 \mathrm{E}-02$ \\
\hline Alzheimer's disease & 61 & $2.12 \mathrm{E}-02$ \\
\hline Circadian rhythm & 9 & $2.15 \mathrm{E}-02$ \\
\hline B cell receptor signaling pathway & 31 & $2.48 \mathrm{E}-02$ \\
\hline Tight junction & 47 & $2.69 \mathrm{E}-02$ \\
\hline Oocyte meiosis & 41 & $2.97 \mathrm{E}-02$ \\
\hline Mismatch repair & 12 & $3.29 \mathrm{E}-02$ \\
\hline Huntington's disease & 60 & $3.33 \mathrm{E}-02$ \\
\hline Arginine and proline metabolism & 22 & $3.73 \mathrm{E}-02$ \\
\hline VEGF signaling pathway & 29 & $3.70 \mathrm{E}-02$ \\
\hline Wnt signaling pathway & 50 & $3.88 \mathrm{E}-02$ \\
\hline Limonene and pinene degradation & 9 & $5.39 \mathrm{E}-02$ \\
\hline Aldosterone-regulated sodium reabsorption & 18 & $5.35 \mathrm{E}-02$ \\
\hline Aminoacyl-tRNA biosynthesis & 18 & $5.35 \mathrm{E}-02$ \\
\hline
\end{tabular}

\subsection{DNA methylation in APP/PS1 mice}

So far, I demonstrated an increased deregulation of gene expression in APP/PS1 mice which is mostly related to immune response. In order to explore the possible mechanisms driving these gene expression changes, I analyzed DNA methylation. DNA methylation is known to influence gene expression in several ways, however, has not yet been significantly explored in the context of $\mathrm{AD}$. To gain some more insight into the molecular processes underlying gene expression in APP/PS1 mice, I performed MeDIP-sequencing on neuronal and non-neuronal cells to analyze DNA methylation along the genome in 1.5, 4, and 8 months old APP/PS1 transgenic mice.

Figure 4.29A (see p.99) shows the average methylation profile of genes in the murine genome for low, mid and high expressed genes based on gene expression data. The number of methylated sites is reduced for all gene fractions around the TSS and methylation in general is reduced in 
the gene bodies of highly expressed genes. This is in line with the general consensus on DNA methylation as a marker for silenced genes. The differences between high and low expressed genes are stable across brain regions and cell types. Approximately $50 \%$ of all sequenced sites of methylation can be mapped to intergenic regions of the genome and 30\% to intronic regions (see figure 4.29B), confirming the function of DNA methylation as a repressive mark of gene expression.

A
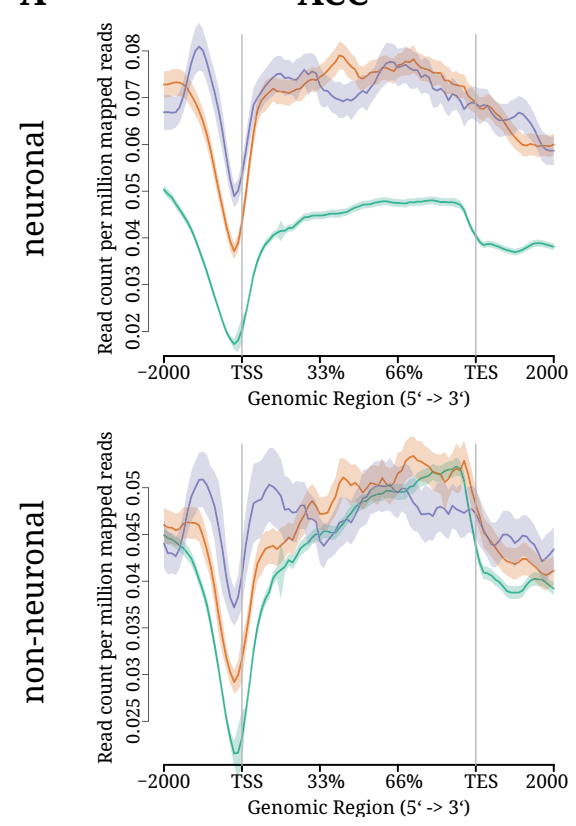

Low expressed.
CA1
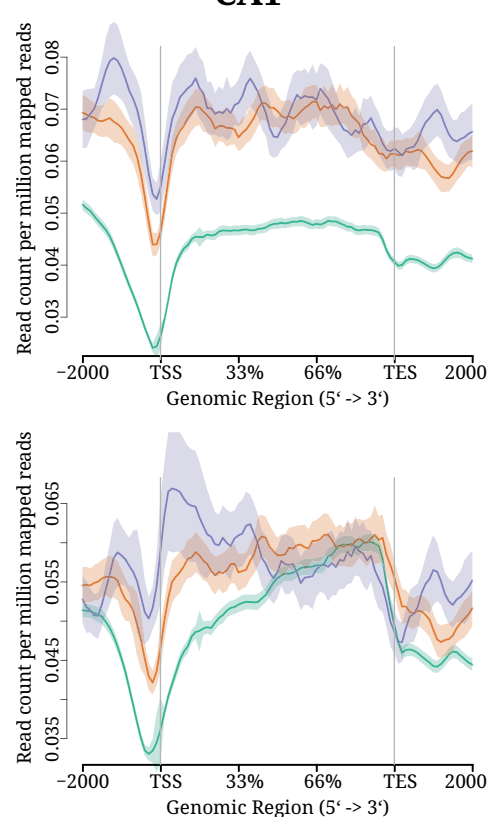

Mid expressed
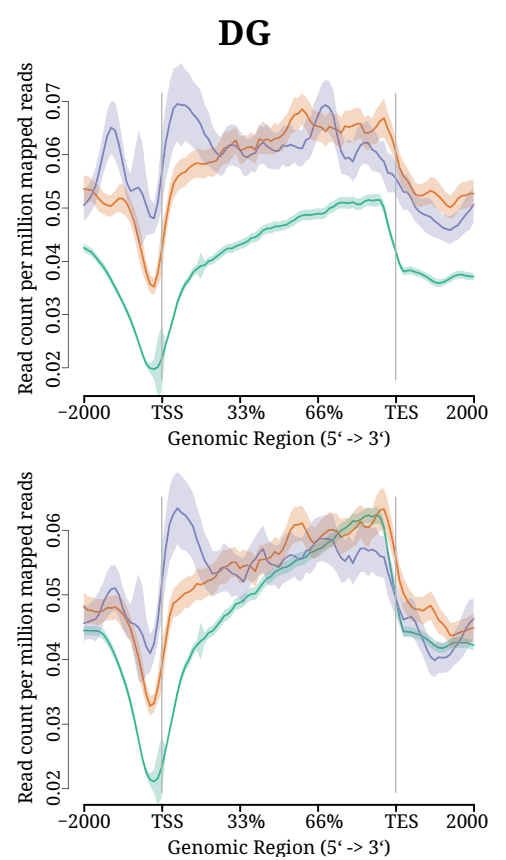

High expressed

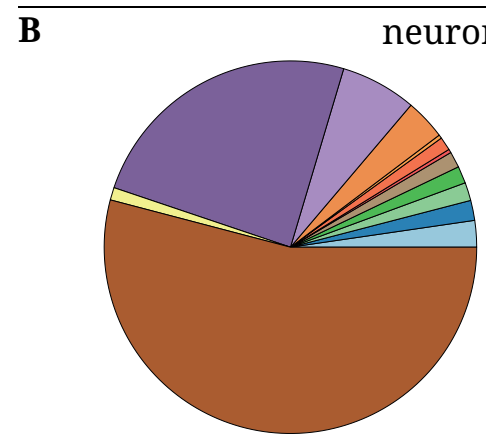

$$
\begin{aligned}
& \square \text { Promoter }(<=1 \mathrm{~kb})(2.31 \%) \\
& \square \text { Promoter }(1-2 \mathrm{~kb})(1.74 \%) \\
& \square \text { Promoter }(2-3 \mathrm{~kb})(1.58 \%) \\
& \square \text { Promoter }(3-4 \mathrm{~kb})(1.48 \%) \\
& \square \text { Promoter }(4-5 \mathrm{~kb})(1.39 \%) \\
& \square 5^{\prime} \text { UTR }(0.31 \%) \\
& \square \text { 3. }^{\prime} \text { UTR }(1.17 \%) \\
& \square \text { 1st Exon }(0.31 \%) \\
& \square \text { Other Exon }(3.52 \%) \\
& \square \text { 1st Intron }(6.58 \%) \\
& \square \text { Other Intron }(24.44 \%) \\
& \square \text { Downstream }(<=3 \mathrm{~kb})(1.09 \%) \\
& \square \text { Distal Intergenic }(54.09 \%)
\end{aligned}
$$

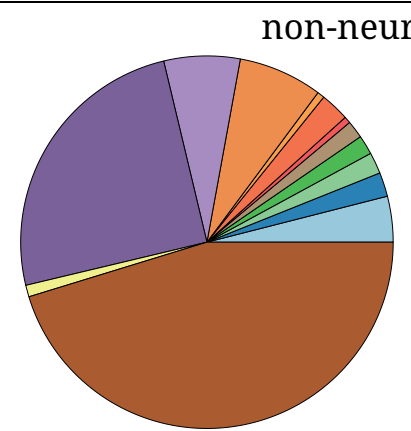

$\square$ Promoter $(<=1 \mathrm{~kb})(3.97 \%)$

口 Promoter (1-2kb) (2.09\%) $\square$ Promoter $(2-3 \mathrm{~kb})(1.85 \%)$ 口 Promoter $(3-4 \mathrm{~kb})(1.67 \%)$ 口 Promoter (4-5kb) (1.53\%) ๑ 5' UTR $(0.57 \%)$ $\square$ 3' UTR $(2.52 \%)$

$\square$ 1st Exon $(0.62 \%)$ Other Exon (7.29\%) $\square$ 1st Intron (6.61\%) - Other Intron $(24.99 \%)$ $\square$ Downstream $(<=3 \mathrm{~kb})(1.01 \%)$ ๑ Distal Intergenic $(45.26 \%)$

Figure 4.29 - NGS plots for DNA methylation along gene bodies:

A) Number of normalized mapped reads along the gene body averaged for the entire genome for 1.5 months old APP/PS1 mice's ACC, CA1 and DG. The three profiles resemble low (purple), mid- (orange) and high (green) expressed genes in each respective brain region. Note the reduced bulk level of DNA methylation in highly expressed genes, resembling their active state.

B) Proportion of each respective genomic methylation site in neuronal and non-neuronal cells. Note that the overall distribution is rather similar but non-neuronal cells had less methylated DNA in intergenic regions. 
A

$$
\text { DNA methylation: }
$$
Gene expression:

ACC

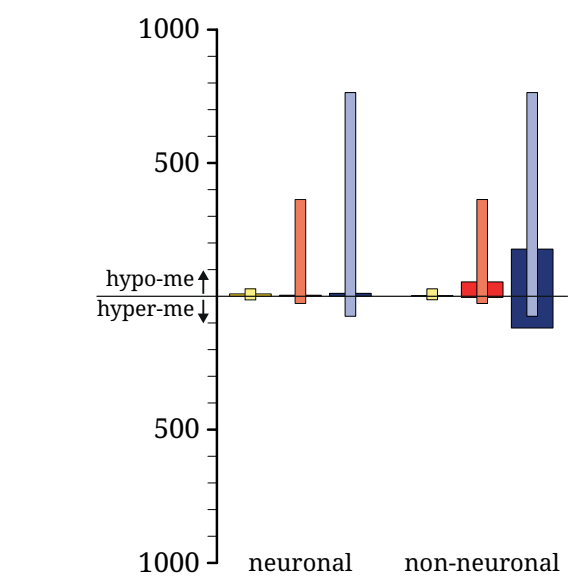

1.5 months 1.5 months

4 months 4 months

CA1

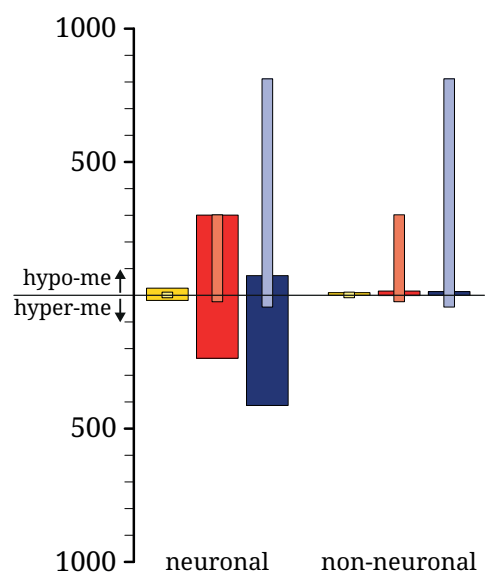

8 months

8 months

DG

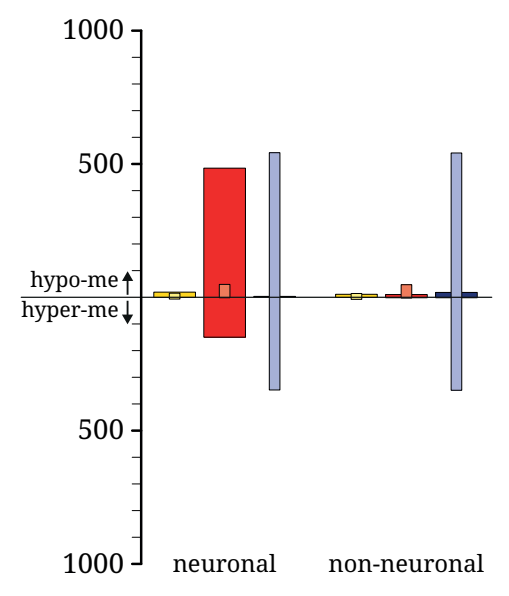

B

\begin{tabular}{r|c|c|c|}
\cline { 2 - 4 } neuron & $1.5 \mathrm{mo}$ & $4 \mathrm{mo}$ & $8 \mathrm{mo}$ \\
\hline ACC & 9 & 4 & 11 \\
\hline CA1 & 46 & 537 & 487 \\
\hline DG & 20 & 634 & 3 \\
\hline
\end{tabular}

\begin{tabular}{r|c|c|c|}
\cline { 2 - 3 } $\begin{array}{r}\text { non- } \\
\text { nuron }\end{array}$ & $1.5 \mathrm{mo}$ & $4 \mathrm{mo}$ & $8 \mathrm{mo}$ \\
\hline ACC & 2 & 58 & 296 \\
\hline CA1 & 10 & 17 & 14 \\
\hline DG & 12 & 13 & 20 \\
\hline
\end{tabular}

C

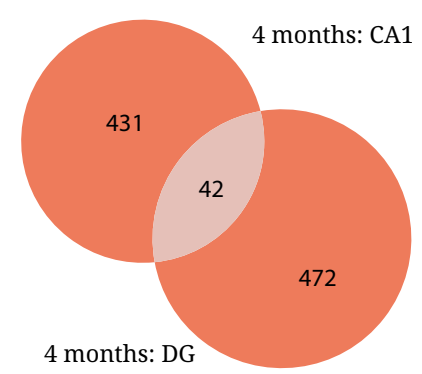

Figure 4.30 - DNA methylation in APP/PS1 transgenic mice:

A) Number of hypo- and hypermethylated 700bp bins (adjusted p-value $\leq 0.2$ ) in neuronal and nonneuronal cells from the ACC, CA1 and DG of APP/PS1 mice and corresponding changes in gene expression (light colors). Values above the $\mathrm{x}$-axis depict downregulation of DNA methylation but upregulation of gene expression and vice versa.

B) Exact numbers of differentially methylated DNA regions in neuronal and non-neuronal cells.

C) Common and region exclusive differentially methylated genes in neuronal cells from the CA1 and DG of 4 month old transgenic mice. Note that the number of genes is only a fraction of the number of significant bins since some genes contain more than one significant methylation site.

Gene expression in 1.5 months old mice was not strongly affected in APP/PS1 mice and thus, one would not expect massive changes in the DNA methylation of those young mice. In fact, only few genomic sites were significantly differentially methylated in the ACC, CA1 and DG of 1.5 months old transgenic mice compared to wildtype littermates (see figure 4.30A and B). Figure 4.30 (A) also shows the number of differentially methylated DNA sites compared to the number of differentially expressed genes in the respective age group and brain region. Surprisingly, the overlap between upregulation of gene expression and DNA hypomethylation is marginal and changes 
in gene expression are clearly exceeding differential DNA methylation. From 246 genes with deregulated DNA methylation sites in the ACC of old mice, only 8 were also differentially expressed in the same age group and brain region (see figure 4.31A, p.102). Similar findings were made for the CA1 and DG. Interestingly, the number of differentially methylated sites increases inconsistently throughout aging in the two cellular fractions while gene expression is disrupted gradually. While there were 296 differentially methylated sites in non-neuronal cells from the ACC of 8 months old mice, differential DNA methylation in neuronal cells or the other age groups is only marginal. An opposite effect can be found in the hippocampal regions CA1 and DG, where most of the differential methylation was found in neuronal cells from 4 months old wildtype vs transgenic mice. Only a smaller fraction of genes that are differentially methylated in either of the regions at 4 months of age is also significant in the respective other hippocampal region (see figure 4.30C, p.100). These findings might indicate differential mechanisms how DNA methylation affects gene expression in distinct cell-types and even brain regions.

Due to the surprisingly low coverage of differential gene expression and DNA methylation, I analyzed the DNA methylation profile of expressed genes in more detail and tried to correlate gene expression with DNA methylation specifically in promoter regions, exons and introns. Figure 4.31B (see p.102) exemplarily shows the results for neurons from the DG of 4 months old mice. No correlation could be found in regard to introns and exons and only a mild correlation can be observed in promoter regions. However, this weak correlation might be artificial due to the redundancy from significant genes as a result from multiple methylation sites within. Apart from a direct impact of DNA methylation on gene expression which could not be confirmed here, it might also function as a priming mechanism for gene expression, thus in a rather long-term pattern. To test this hypothesis, I compared DNA methylation from 4 months old mice with data from RNA sequencing from 8 months old mice and again found no decent correlation (see figure 4.31C, p.102). Furthermore, genes with differentially methylated DNA sites can not be assign to common functional pathways, supporting the previous findings of an absent or only mild direct connection between DNA methylation and differential gene expression.

These results indicate, that DNA methylation is not affected in those genes differentially expressed in APP/PS1 transgenic mice and that the effect of DNA methylation on gene expression in APP/PS1 mice might not primarily depend on intragenic mechanisms. 
A

ACC

non-neuronal, 8 months

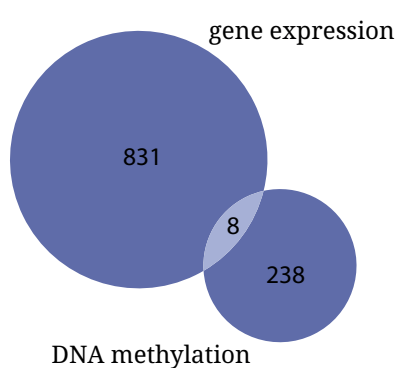

CA1

neuronal, 4 months

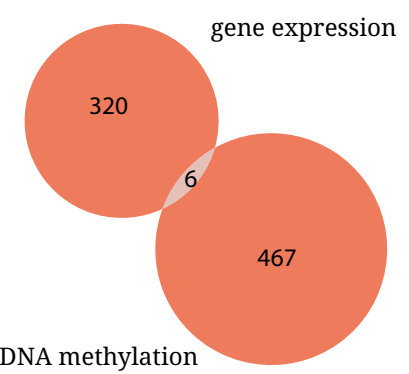

DG

neuronal, 4 months

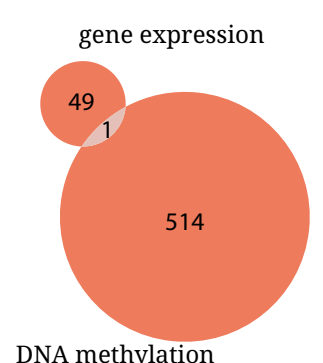

B

Promoter

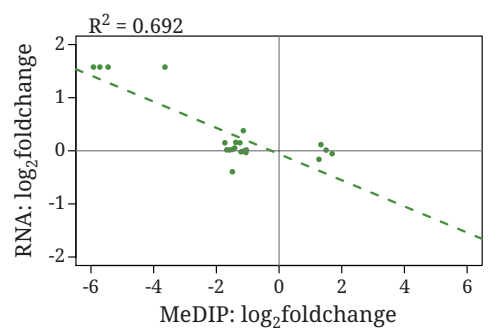

C

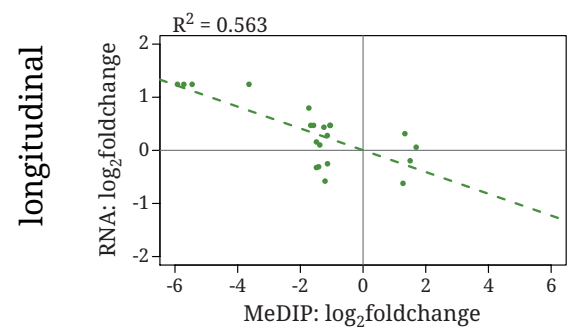

Exon
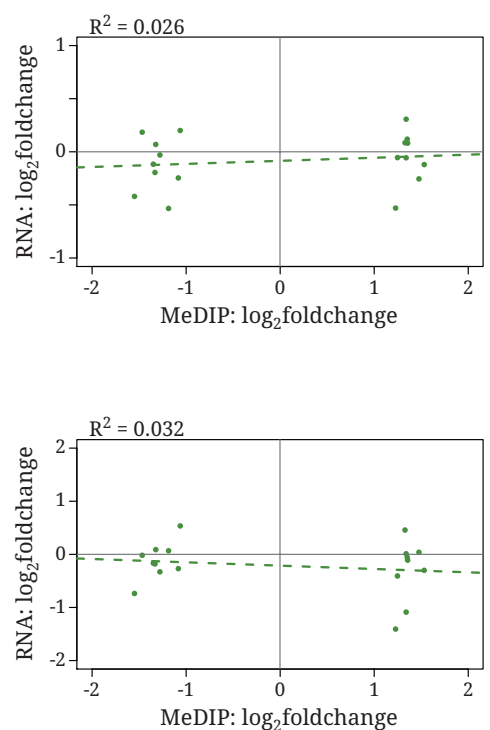

Intron
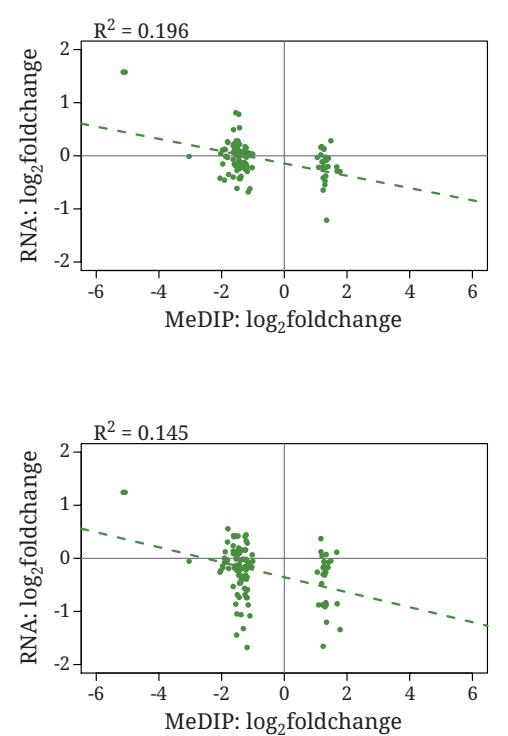

Figure 4.31 - Independence of differential gene expression and DNA methylation in APP/PS1 mice: A) Overlap of deregulated gene expression and differentially methylated DNA sites (padj $\leq 0.2$ ) annotated to genes in non-neuronal cells from the ACC of 8 months old mice and neuronal cells from the CA1 and DG of 4 months old mice.

B) Absent correlation between the foldchanges from MeDIP- and RNA-seq data analysis for promoter (left), exonic (center) and intronic (right) regions. Dashed lines resemble a linear regression of data. The $\mathrm{R}^{2}$-value for each correlation is depicted on top of each plot. Example data shown represent neuronal tissue in the DG of 4 months old transgenic mice compared to wildtype littermates.

C) Similar correlation analysis regarding the same MeDIP data as shown in B and RNAseq data from 8 months old mice's DG.

The absent correlation of DNA methylation and differential gene expression indicate, that DNA methylation might be rather globally affected and not specific to certain genes. When comparing the genome-wide average read count along the gene body, the profiles in cells from the different brain regions and cell-types are rather diverse. Though a considerable amount of DNA sites were 
ACC

non-neuronal, 8 months

A

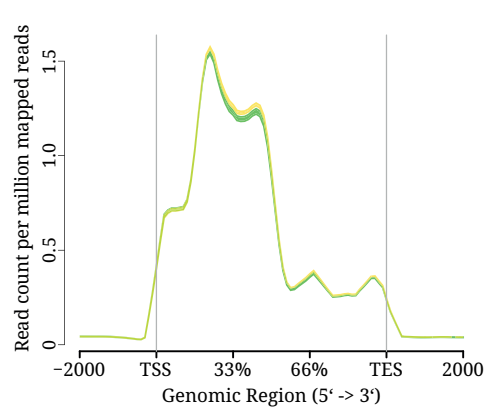

B
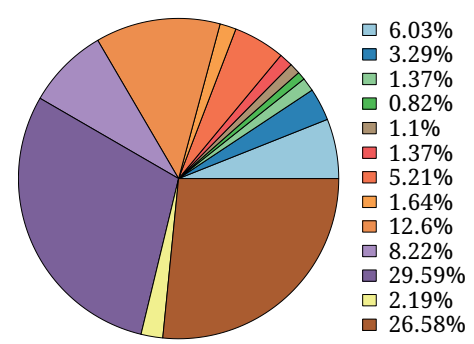

$\square .03 \%$
$\square 3.29 \%$

$\square 1.37 \%$

$\square 0.82 \%$

$\square 1.1 \%$

- $1.37 \%$

$\square$
$\square 5.21 \%$

$\square 1.64 \%$

$\square 12.6 \%$

$\square 8.22 \%$

$\square 29.59 \%$

ㅁ $2.19 \%$

Promoter $(\leq 1 \mathrm{~kb})$

Promoter (1-2kb)

Promoter (2-3kb)
CA1

neuronal, 4 months
DG

Transgenic

neuronal, 4 months
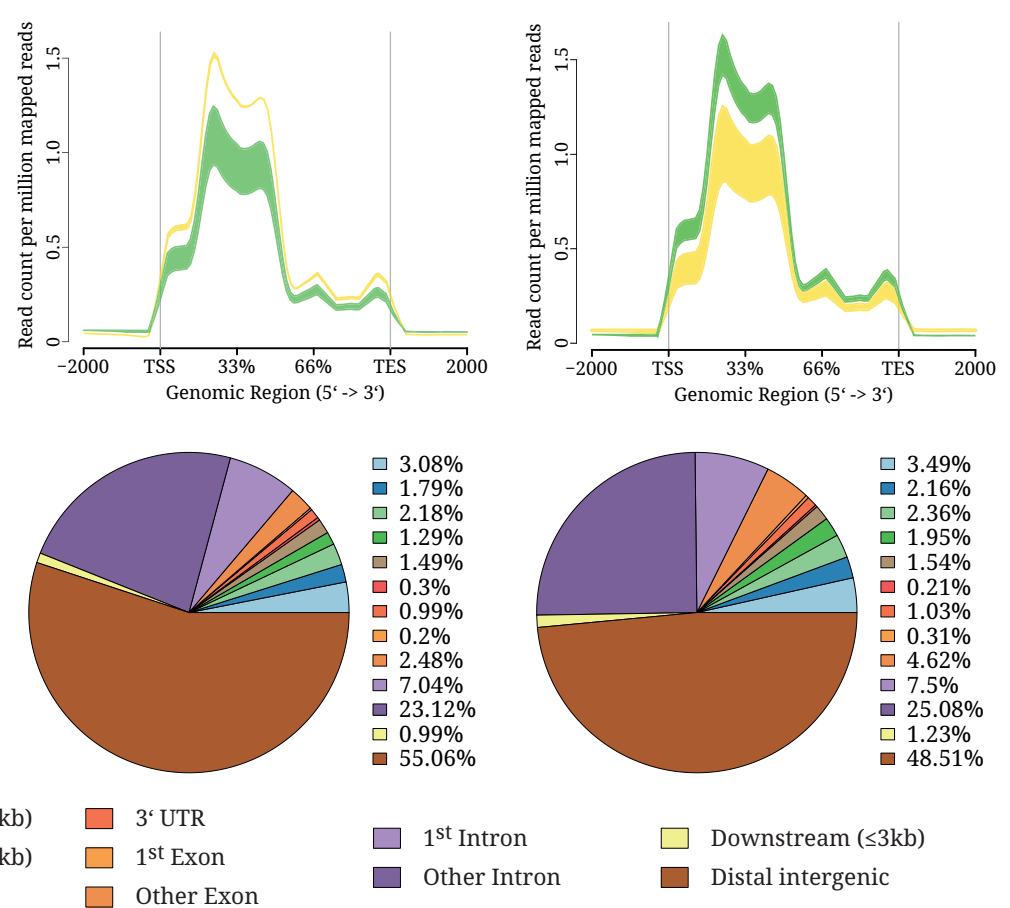

$1^{\text {st }}$ Intron

Other Intron

Downstream $(\leq 3 \mathrm{~kb})$

Distal intergenic

Figure 4.32 - Differential distribution of DNA methylation in APP/PS1 mice:

A) Number of normalized mapped reads along the gene body averaged for the entire genome for samples with the highest number of differentially methylated DNA sites respectively for the ACC, CA1, and DG of APP/PS1 wildtype (green) and transgenic (yellow ) mice. The profiles resemble the range of means for the respective replicates.

B) Distribution of differentially methylated DNA sites along the genome. Note that the overall profile is rather similar to the baseline distribution with the exception of non-neuronal cells in the ACC.

deregulated in non-neuronal cells from the ACC of old mice, no difference between wildtype and transgenic mice can be detected on a genome-wide scale (see figure 4.32A). Interestingly, DNA methylation is globally upregulated in CA1-neurons from transgenic mice while an opposing effect can be observed in the DG. These findings emphasize the differential linkage between DNA methylation and gene expression in different cellular states and environments.

Compared to the global distribution of DNA methylation in either neuronal or non-neuronal cells (see figure 4.29B, p.99), differentially methylated sites in non-neuronal cells from the ACC of 8 months old mice are enriched inside of the gene body, predominantly within promoters, exons, and 5' and 3' untranslated regions (see figure 4.32C). Methylation within introns, in contrast, remains relatively stable. This enrichment is absent in neuronal cells from the CA1 and 
DG, where the distribution of significant methylation sites is rather similar to the global distribution of DNA methylation with the majority of significant sites mapping to intergenic regions. These data suggest a partially gene-directed effect in glial cells and a rather global disruption of DNA methylation in neuronal cells which might explain the absence of significant functional pathways represented by the differentially methylated genes.

ACC non-neuronal, 8 months
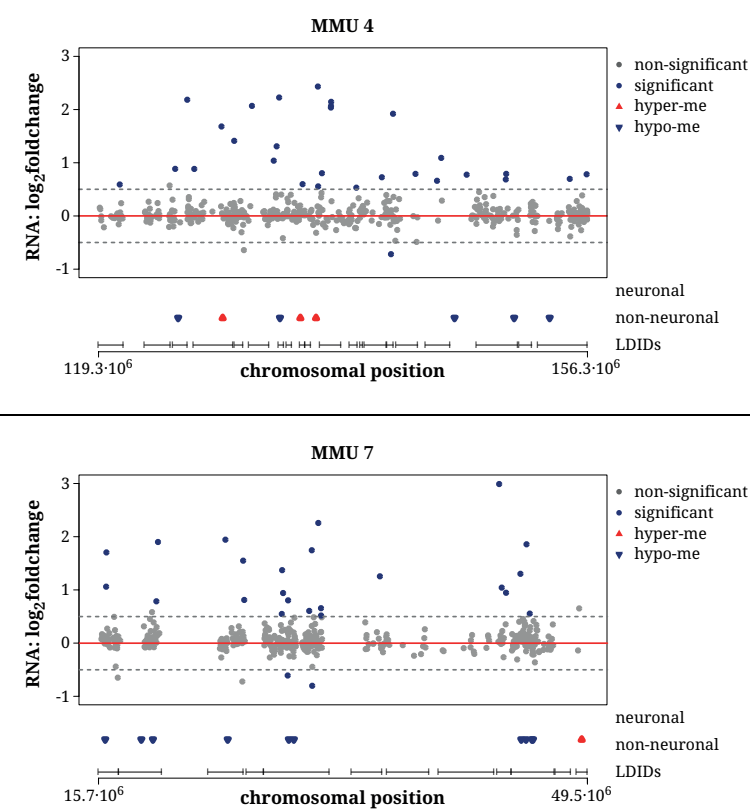

CA1

neuronal, 4 months
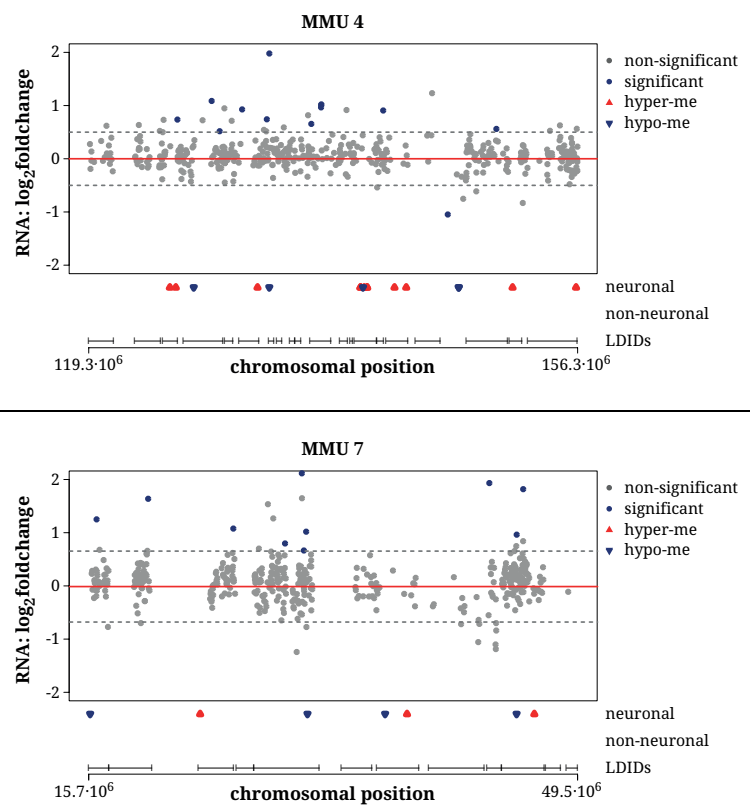

Figure 4.33 - Long distance interactions between genes and sites of DNA methylation:

Genes identified via RNA sequencing from the ACC of 8 months old mice and CA1 of 4 months old mice plotted for defined regions of chromosome MMU4 and MMU7. Significantly differentially expressed genes are labeled blue, non-significant ones in gray. Hypo- (blue) and hypermethylated (red) DNA sites are depicted as rectangles below each graph. Previously described long distance interaction domains (LDID) were added to identify correlations between gene expression changes and distant DNA methylation.

Due to the high proportion of significant intronic and intergenic methylation sites, I hypothesized that the effect of the transgene on DNA methylation is not specifically directed towards significantly deregulated genes identified by RNA sequencing but rather functioning on distant interactions. Therefore, I aimed to identify deregulated genes and DNA methylation sites within previously described long distance interaction domains (Dixon et al., 2012). In those groups showing a strong deregulation of DNA methylation, $68.1 \%$ to $75.5 \%$ of all deregulated genes were found in domains containing differentially methylated DNA sites. Figure 4.33 shows defined regions of the murine chromosomes 4 and 7 and the respective results from non-neuronal cells 
from the ACC and CA1-neurons. Notably, though one can identify common domains of deregulated genes and methylation sites, the number of affected genes per domain is rather little. I thus screened for gene expression hot-spots, defined as long distance interaction domains in which at least $20 \%$ of the expressed genes are differential in APP/PS1 mice. Notably, only 7 hot-spots were identified in the ACC and CA1 of old mice and 2 in the CA1 of 4 months old mice (see table 7.18, p.136). DNA methylation changes in these regions was only sparse as it is exemplarily shown in figure 4.34 .

ACC non-neuronal, 8 months

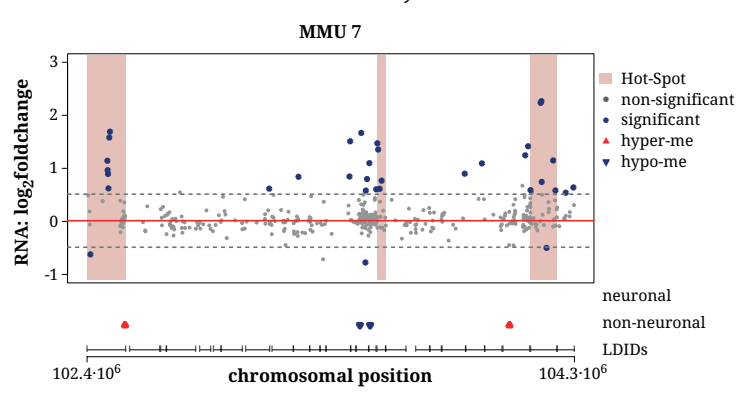

\section{CA1}

neuronal, 4 months

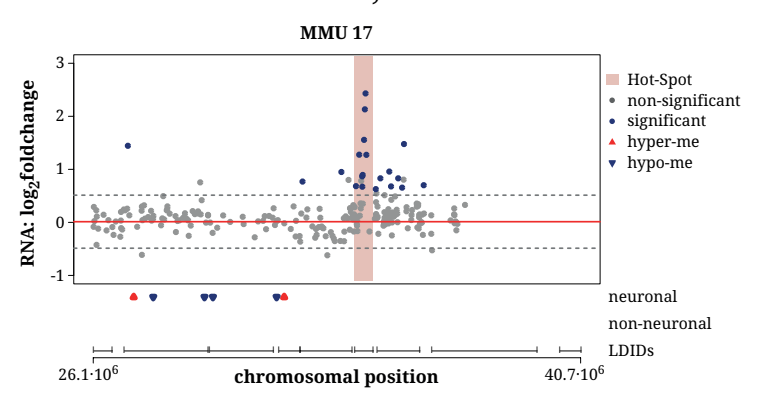

Figure 4.34 - Gene expression hot-spots and sites of DNA methylation:

Hot-spots within the murine chromosome 7 and 17 in the ACC and CA1 of 8 and 4 months old mice respectively. Long distance interaction domains with at least $20 \%$ of the genes within deregulated in APP/PS1 mice are here defined as hot-spots (light red). Significantly deregulated genes are labeled blue and hypo- (blue) and hypermethylated (red) DNA sites are depicted as rectangles underneath the plot as well as the LDIDs within the given area of the chromosome.

Taken together, data from MeDIP sequencing suggest that DNA methylation changes in APP/PS1 mice are not directly affecting gene expression via intragenic nor intrachromosomal interactions. These findings, however, oppose the general consensus that a high DNA methylation state is directly linked to a low gene expression rate and vice versa. I thus speculated, that DNA methylation per se is rather defining a basal gene expression - i.e. that defining cell fate - than acting on gene expression upon endogenous stimuli. To test this hypothesis, I compared the DNA methylation profiles of neuronal and non-neuronal cells in 1.5 months old mice to avoid any effect of aging or amyloid pathology. The observed differences between neuronal and non-neuronal cells are massive and range from approximately 10,000 in the DG to more than 30,000 differentially methylated sites in the CA1 and ACC of 1.5 months old mice. This further supports the described findings and indicates the importance for DNA methylation in determining cell fate. 


\subsection{H3K4me3 in APP/PS1 mice}

The strong induction in gene expression of APP/PS1 transgenic mice regarding immune response might be encoded by a downregulation of gene repressive marks and an upregulation of active marks. One histone modification that is in general associated with active genes in H3K4me3. Thus, I performed a ChIPseq analysis on H3K4me3 to reveal changes in this particular epigenetic marker and to find a potential correlation to gene expression. Due to the previously discovered diverse cell type specific effect of amyloid deposits on DNA methylation regarding the ACC and the hippocampal regions, I used the the ACC and DG for this subsequent analysis.

A
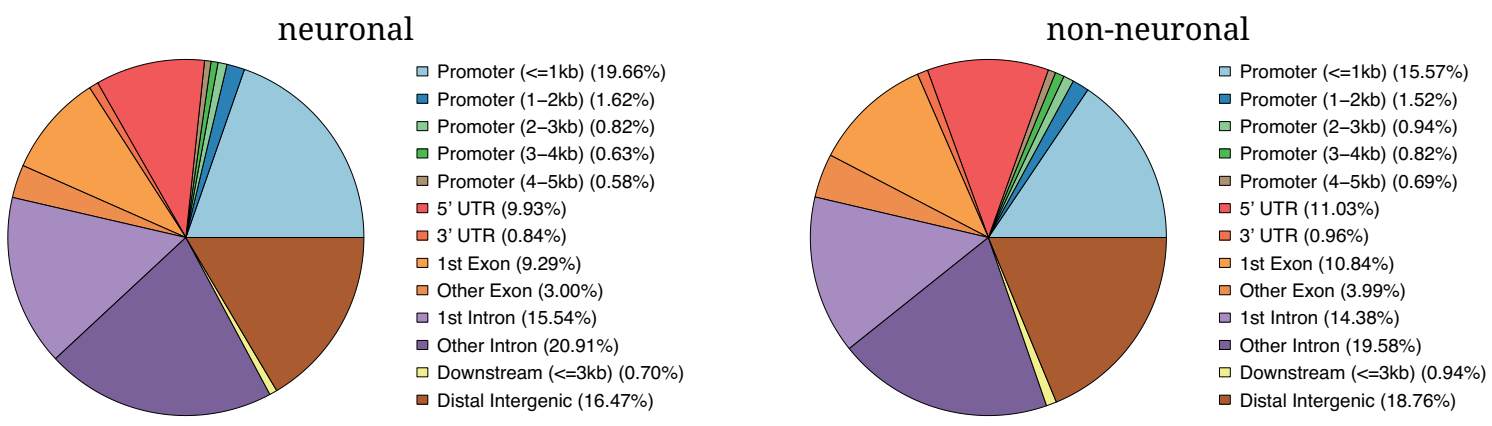

B

C
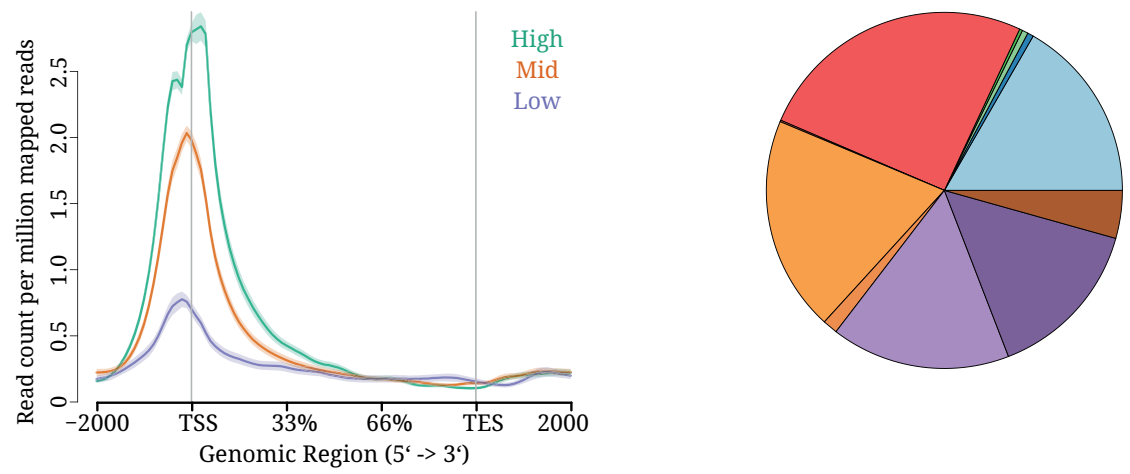

ㅁ Promoter $(<=1 \mathrm{~kb})(16.69 \%)$

口 Promoter $(1-2 \mathrm{~kb})(0.54 \%)$ 口 Promoter $(2-3 \mathrm{~kb})(0.54 \%)$ 口 Promoter (3-4kb) $(0.27 \%)$ $\square$ Promoter $(4-5 \mathrm{~kb})(0.00 \%)$ ㄷ 5' UTR $(25.51 \%)$ 口 3' UTR $(0.14 \%)$ ㅁ 1st Exon (19.54\%) $\square$ Other Exon (1.36\%) ㅁ 1st Intron (16.28\%) - Other Intron (14.79\%) $\square$ Downstream (<=3kb) $(0.00 \%)$ - Distal Intergenic (4.34\%)

Figure 4.35 - Genome wide distribution of H3K4me3:

A) Relative occurrence of H3K4me3 peaks within the gene body and in distal intergenic regions. Data shown are for the DG of 4 months old mice and exemplary for all age groups, cell types and brain regions. B) Number of normalized mapped reads along the gene body averaged for the entire genome. The three profiles shown resemble low(purple), mid (orange), and high (green) expressed genes.

C) Occurence from all significant H3K4me3 peaks (adjusted p-value $\leq 0.2$ ) in non-neuronal cells from the DG of 4 months old mice. Note an enrichment of signals within the 5'-untranslated region and first exon. 
Strikingly, only a single gene with differentially tri-methylated H3K4 was found in neurons from aged mice in the ACC and the DG considering an adjusted p-value $\leq 0.2$, namely Thy1. Surprisingly, a higher number of significant sites (737) was found in non-neuronal cells from the DG of 4 months old mice, corresponding to 501 individual genes. H3K4me3 is a well accepted mark for active promoters and was thus expected to change concordantly to the induction of immune response related gene expression. Figure 4.35A (see p.106) shows the genome wide distribution of peaks which were most abundant within promoter and intronic regions. Also in line with the previous assumptions is the induction of H3K4me3 in proximity to the TSS of highly expressed genes (see figure 4.35B, p.106). Interestingly, the significant differences concerning H3K4me3 in the DG of 4 months old mice showed a strong enrichment of signal in the 5'UTR and first exons indicating an effect on initiating transcription and presumably driving changes in gene expression as indicated in figure $4.35 \mathrm{C}$ (see p.106).

A

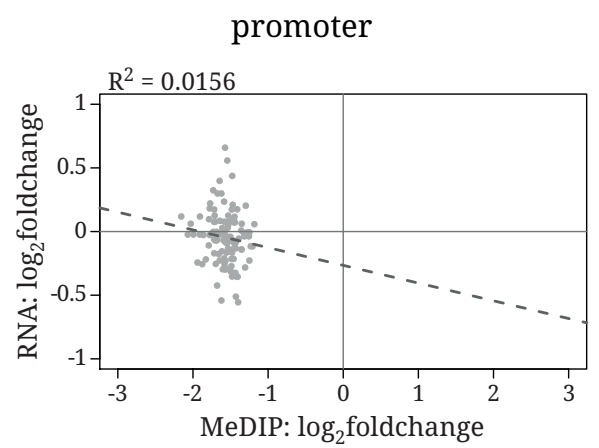

intron

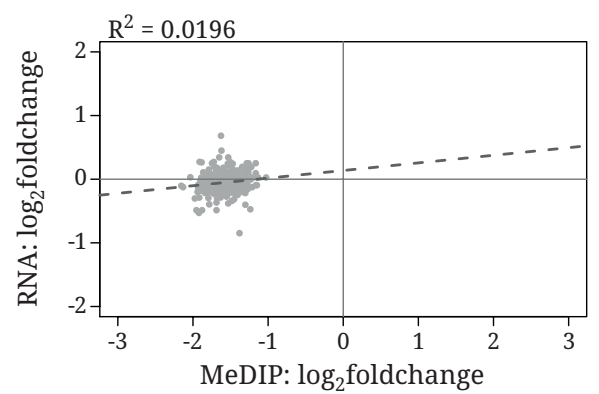

exon

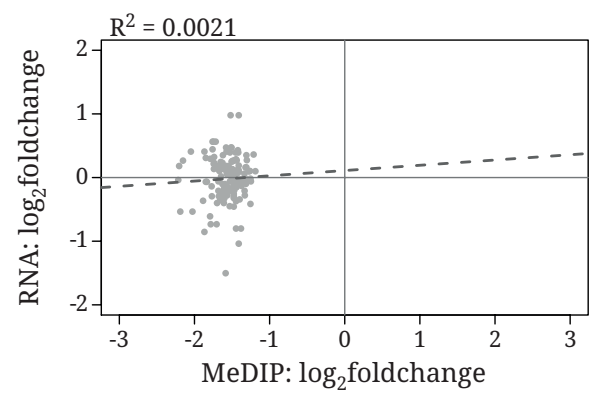

B

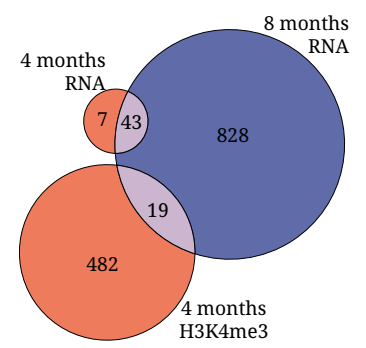

Figure 4.36 - Independence of identified H3K4me3 peaks and differential gene expression in APP/PS1 mice:

A) Absent correlation between H3K4me3 and differential gene expression in non-neuronal DG-cells from 4 months old APP/PS1 mice concerning promoter regions, exons and introns.

B) Venn diagram for overlaps between RNAsequencing from 4 and 8 months old mice and H3K4me3 ChIPseq from 4 months old mice. Note that not a single gene differentially express in mid-aged mice showed a significantly differential H3K4me3 signal. 
The changes observed in H3K4me3, however, only reflect downregulation of histone methylation and are independent from differential gene expression, indicating an artificial effect within the data which might partially result from the relatively low sample size (see figure 4.36, p.107). Taken together, data from H3K4me3-ChIPseq in general could confirm the common assumptions on that particular histone mark functioning in transcription initiation. However, the low sample size and relatively high variance within samples preclude a thorough analysis on amyloidosis driven effects on histone methylation.

\subsection{Drug screening for novel therapeutic strategies in AD}

As I was able to show, the transcription machinery is strongly disrupted in APP/PS1 mice coinciding with amyloid pathology. This diruption might be partially driven by deregulated TFs and epigenetic modification that might act directly on the respective genes or via long-distance interactions. Thus, the data from APP/PS1 mice do partially match data from human AD patients and might thus serve as a valid model to predict novel therapeutic strategies. To do so, I performed a drug screening using the DrugPairSeeker. Since no significant pathology-related changes were found for alternative splicing and, in addition, the effect of DNA methylation remains unclear, the drug screening was based on gene expression data.

The DrugPairSeeker is a Java-based tool developed and published by Zhong et al. (2013). It compares a given set of up- and downregulated genes with a differential gene expression database retrieved for several cancer cell lines treated with more than 1300 individual drugs. It computes the overlay of genes upregulated in the context of a certain phenotype and those downregulated in the database and vice versa. The difference between matches and mismatches is taken as a score for each respective drug. Thereby, the DrugPairSeeker tries to predict the most efficient pair of drugs which might attenuate the observed deregulation of gene expression. For identifying novel drugs, I ran the DrugPairSeeker with all genes from the respective comparisons with the given thresholds for significance and foldchange. Since amyloid pathology is acting on all three tested brain regions, affecting both hippocampal and cortical dependent cognition, the top 10 ranked drug pairs for each comparison (Wildtype vs transgenic and aging in transgenic mice) were collected. Drugs were then ranked according to their frequency in the given comparisons 
and were filtered to remove non-promising drugs. Drugs were filtered to exclude those that show adverse site effects, are unlikely to cross the blood brain barrier, were withdrawn by the World Health Organisation, or were already unsuccessfully tested in regard to AD.

The resulting top 10 drugs are listed in table 4.10. Even though the DrugPairSeeker's database was built on different cancer cell lines and not in regard to neuropathologies, part of these highly ranked drugs do actually seem promising for the treatment of AD. Three of the top 10 compounds are acting as HDAC inhibitors. HDAC inhibitors like vorinostat (SAHA) were already considered as promising therapeutic drugs for Alzheimer's disease and successfully tested by our group in the past in regard to $\mathrm{AD}$ and age-related cognitive decline (Benito et al., 2015; Peleg et al., 2010; Stilling et al., 2014). The drug screening was based on the differential gene expression and results do thus mainly cover changes in immune response observed in APP/PS1 mice. Concordantly, other highly ranked drugs were shown to act in the suppression of $\mathrm{NF}_{\mathrm{K}} \mathrm{B}$ signaling (Acacetin and Parthenolide) or in maintaining homeostasis (i.e. Kaempferol). Another drug that was discovered by the DrugPairSeeker though not among the top10 is Memantine, a NDMA receptor antagonist currently used for the treatment of progressed AD. As an antagonist for NMDA receptors, it is mainly functioning in neuronal signaling and plasticity.

Table 4.10 - Top 10 drugs identified by screening with DrugPairSeeker after filtering

\begin{tabular}{|c|c|c|c|}
\hline Drug & chemical family & source & mode of action \\
\hline Vorinostat (SAHA) & hydroxamid acid & synthetic & HDAC inhibitor \\
\hline Trichostatin A & benzendoid & $\begin{array}{l}\text { natural (i.e. Strepto- } \\
\text { myces platensis) }\end{array}$ & HDAC inhibitor \\
\hline Acacetin & flavone & $\begin{array}{l}\text { natural (i.e. Robinia } \\
\text { pseudoacacia) }\end{array}$ & NF $\kappa$ B suppression \\
\hline Parthenolide & lactone & $\begin{array}{l}\text { natural (i.e. Tanace- } \\
\text { tum parthenium) }\end{array}$ & NF $\kappa$ B suppression \\
\hline Pirinixid acid & pyrimidine derivate & synthetic & PPAR $\alpha$ antagonist \\
\hline Piperlongumine & pyridin derivate & $\begin{array}{l}\text { natural (i.e. Piper } \\
\text { longum) }\end{array}$ & reactive oxygen species induction \\
\hline Lovastatin & lactone & $\begin{array}{l}\text { natural (i.e. } \\
\text { pergillus terreus) }\end{array}$ & HMG-CoA-reductase inhibitor \\
\hline Sirolimus & macroldie & $\begin{array}{l}\text { natural (i.e. Strepto- } \\
\text { myces hygroscopicus) }\end{array}$ & mTOR inhibitor \\
\hline Valproic acid & fatty acyl & synthetic & HDAC inhibitor \\
\hline Kaempferol & flavone & natural (i.e. Aloe vera) & antioxidant \\
\hline
\end{tabular}




\subsection{Combinatory treatment with SAHA and memantine}

For identifying a novel therapeutic approach to counteract AD, I aimed for a targeting both the strong immune response and the mild deregulation of plasticity related genes found in APP/PS1 transgenic mice. Thus, I hypothesized that a combination therapy of the two individually well described drugs Memantine and Vorinostat might augment their respective effect on cognition and amyloid pathology. Dosages were chosen where the full therapeutic potential of each individual drug is not reached to enable the identification of mild enhancements of a combinatory treatment. Transgenic mice of 4-5 months of age were treated with the drug-combination for 4 weeks before behavioral experiments started. The age group of 4-5 months was chosen to model a stage of the disease in human patients, where it can be diagnosed and thus a therapy can be initiated. Mice underwent the open field test, elevated plus maze, Morris water maze and fear conditioning to discover potential differences in hippocampus and cortex related learning and memory.

A

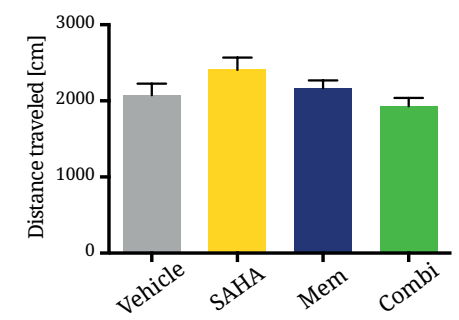

B

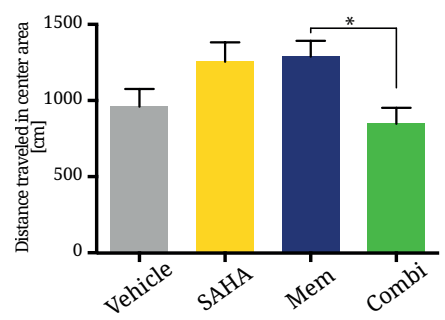

C

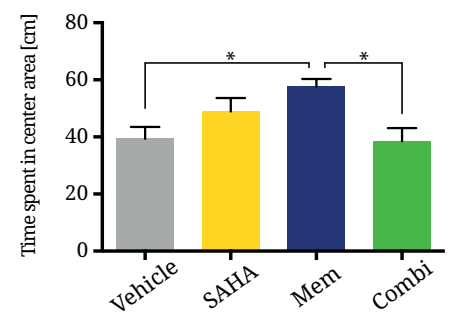

Figure 4.37 - Explorative behavior and basal anxiety levels in drug treated APP/PS1 transgenic mice: A) Overall distance traveled inside the open field arena. A reduction in that value would indicate a reduction in exploratory behavior. No difference could be observed between the groups (ANOVA: $p=0.082$ ) B) The distance traveled inside the center area $(10 \mathrm{~cm}$ distance from the walls) as a read-out for basal anxiety. None of the groups showed a significant increase compared to the vehicle group, though Memantinetreated mice spent more time in the center area than mice from the Combi-group (Tukey's test: $p=0.036$ ). C) Time spent inside the center area. Mice treated with memantine (Mem) showed a significant increase compared to mice treated with vehicle $(p=0.036)$ and combinatory treatment $(p=0.014)$ indicating a reduced basal anxiety.

The open field test is a behavioral experiment to quantify locomotive, exploratory and basal anxiety related behavior in mice. Mice treated either with SAHA, memantine or a combination of both are not affected in their locomotion, as mice from all groups traveled approximately the same distance within the open field area (see figure 4.37A). The time spent and distance traveled 
within the center area of the open field are indicators for basal anxiety levels. Mice treated with memantine spent significantly more time in the center area than mice treated with vehicle $(\mathrm{p}=$ 0.0359) or the combinatory treatment $(p=0.0140)$ and traveled a longer distance in the center area than mice that received the combinatory treatment $(p=0.0362)$ while no significant effect was found after treatment with SAHA, neither alone nor in combination with memantine.

A second test for studying anxiety-like behavior in mice is the elevated plus maze. A tendency of mice to stay in the closed arms of the plus maze is an indicator for an increased anxiety. However, none of the groups showed a significant effect in the elevated plus maze compared to mice treated with vehicle, neither regarding the time mice spent in open or closed arms nor the latency to enter either of the arms the first time ( $p>0.05)$ (see figure 4.38). Thus, the mild anxiolytic effect that was found for memantine when given exclusively in the open field test could not be confirmed in the elevated plus maze.

A

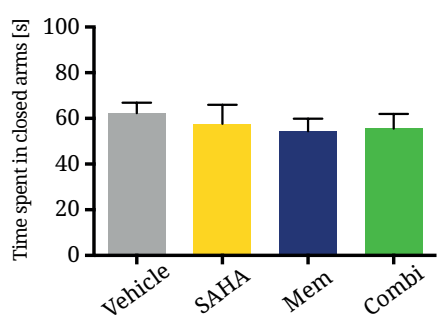

B

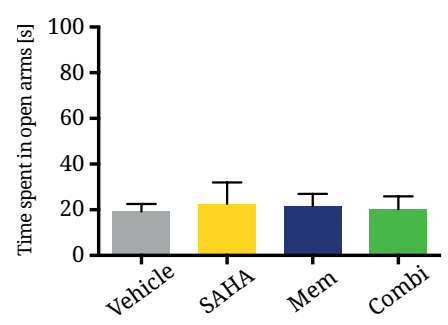

C

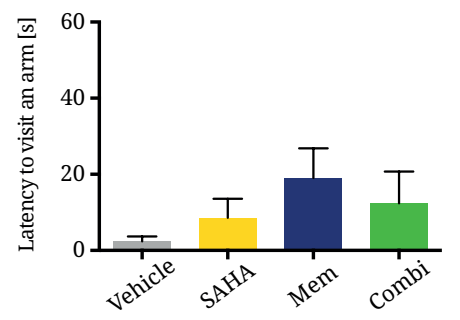

Figure 4.38 - Evaluation of anxiety-like behavior in treated APP/PS1 transgenic mice:

A \& B) Total time the mice spent inside the closed arms (A) and on the open arms (B). A tendency towards open arms would indicate an anxiolytic effect of the respective medications, though no significant differences were found between the groups (ANOVA: $\mathrm{p}_{A}=0.85, \mathrm{p}_{B}=0.98$ ).

C) Latency for the first exploration of either arm. A significant difference could mask results from the ratio of time spent in open and closed arms. No significant differences were detected among the tested mice (ANOVA: $\mathrm{p}=0.43$ ).

To study spatial memory, mice were tested in the Morris water maze. Figure 4.39A (see p.112) shows the learning curve based on the time the mice needed on each consecutive day to find the platform. The strongest learning effect can be seen during the first 3 days of training and mice performed significantly better in finding the platform after 10 days $(p<0.0001)$. The only significant difference in learning compared to mice treated with vehicle was observed at day 3 , when SAHA mice performed substantially worse $(p=0.0167)$, and on day 7 when mice treated with memantine showed a reduced escape latency and thus a better learning $(p=0.0163)$. A first probe test was performed at day 6 when the learning curve seemed to have reached saturation. 
A

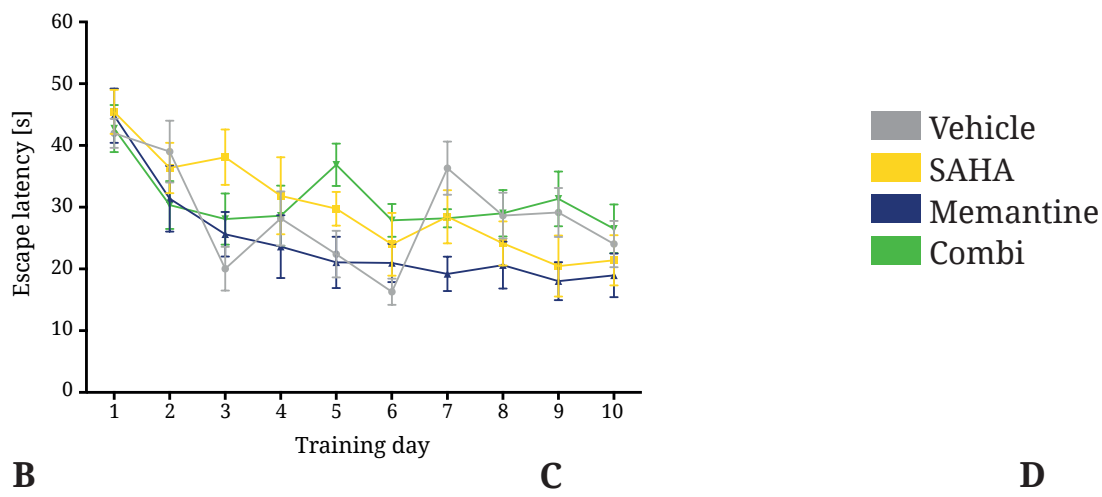

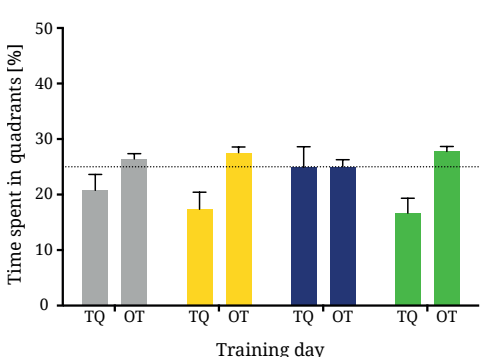

$\mathbf{E}$

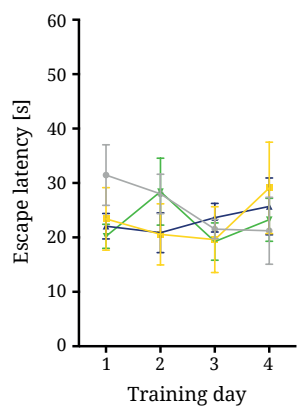

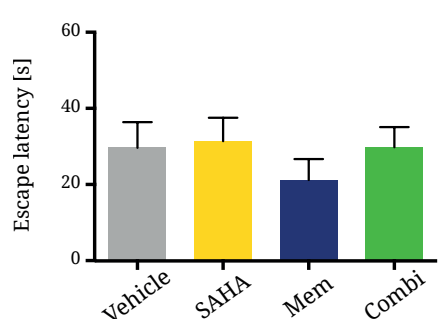

$\mathbf{F}$

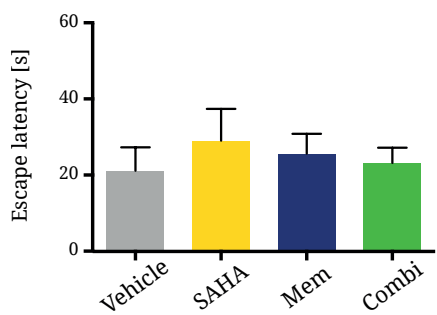

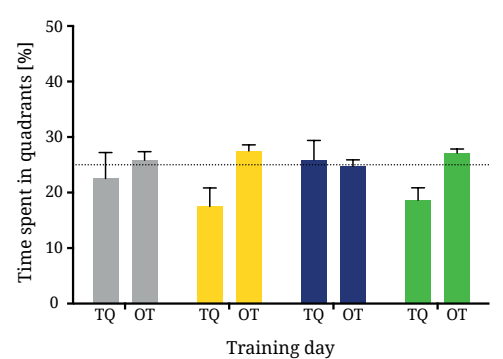

G

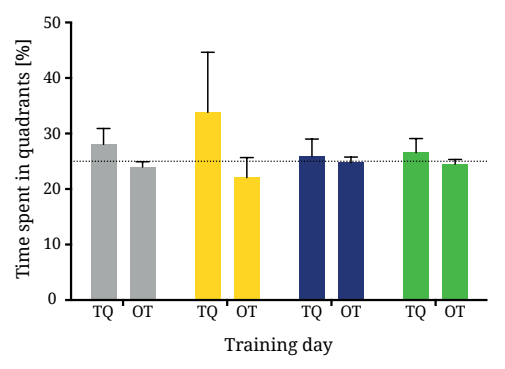

Figure 4.39 - Spatial memory in treated APP/PS1 mice tested in the Morris Water Maze:

A) Average time needed to enter the target platform on each consecutive training day. Learning effect was confirmed by 2-way ANOVA ( $<<0.0001)$. Significant difference towards vehicle were found in the SAHA group at day $3(p=0.0167)$ and in the memantine group at day $7(p=0.0163)$.

B) Percentage of time mice spent in the target quadrant (TQ) and off-target quadrants (OT) during the first probe test at day 6 . Chance level of $25 \%$ is resembled by the dashed line.

C) Latency to cross the target platform area during the second probe test at day 11. No significant differences were found among the groups (ANOVA: $p=0.62$ ).

D) Percentage of time mice spent in the target quadrant (TQ) and off-target quadrants (OT) during the second probe test at day 11 . Chance level of $25 \%$ is resembled by the dashed line.

E) Average time needed to enter the target platform on each consecutive training day during reversal learning. No significant improvements were observed for the 4 days of reversal training (2-way ANOVA: $p_{\text {days }}=$ $0.64)$.

F) Escape latency during the probe test after reversal learning. No significant differences were observed among the groups (ANOVA: $\mathrm{p}=0.86$ ).

G) Percentage of time spent in the target quadrant (TQ) and off-target quadrant (OT) during reversal probe test. Chance level of $25 \%$ is resembled by the dashed line. 
None of the groups showed a significant preference for the target quadrant and, interestingly, similar findings were made even five days later when performing a second probe test (see figure 4.39B-D, p.112). This indicates that all mice showed severe memory impairments, though they have improved in performing the task initially. Since no significant enhancement could be detected regarding spatial memory retrieval, reversal learning test was performed to test for a potential effect on cognitive plasticity. Therefore, the platform was placed in the opposite quadrant and mice were trained for another 4 days. As it is shown in figure 4.39E (see p.112), mice have already learned the new platform position at the first day as the escape latency did not differ from the last day of initial training. No further significant improvement of the latency was discovered for any of the groups (2-way ANOVA: $p_{\text {days }}=0.64$ ). The probe test after reversal learning revealed again no difference between drug treated mice and those treated with vehicle (ANOVA: $p=0.86$ ). Also, even though all groups did reach the chance level of $25 \%$, none of the groups showed a significant preference towards the novel target quadrant (two-tailed t-test: $\mathrm{p}$ $>$ 0.7). Memantine or SAHA when given alone or in combination were thus at the given dosage not suitable to reduce the cognitive impairment in APP/PS1 mice regarding the acquisition and retrieval of spatial memory.

A

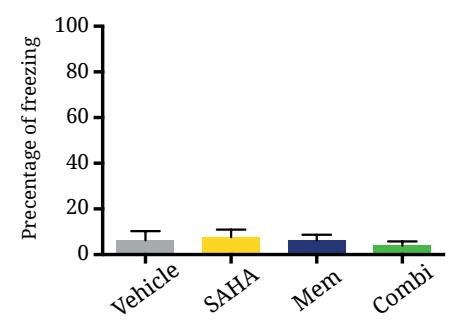

B

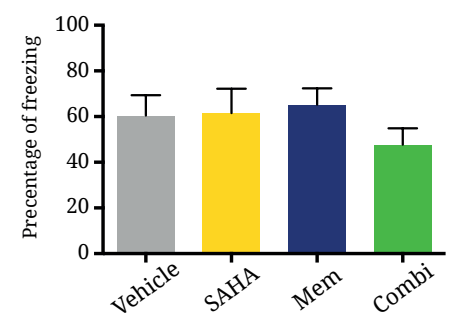

Figure 4.40 - Contextual fear conditioning in APP/PS1 mice after drug treatment:

A) Percentage of time mice spent with freezing behavior during conditioning prior to the electric foot shock. No significant differences were observed (ANOVA: $p=0.80$ ).

B) Percentage of time mice spent with freezing behavior 24 hours after conditioning. No differences can be found between the groups (ANOVA: $\mathrm{p}=0.39$ ).

Finally, I tested the mice in the contextual fear conditioning to quantify a potential effect of either drug on the formation and/or retrieval of contextual aversive memory. During the conditioning trial and, thus, prior to the test, none of the groups showed any difference regarding the overall motility compared to vehicle mice (see figure 4.40A), matching the findings from open field (see figure 4.37A, p.110). All mice have learned the foot shock and were showing a considerable amount of freezing behavior 24 hours after the test when being reintroduced to the conditioning 
chamber (see figure 4.40B, p.113), however no significant differences could be observed (ANOVA: $\mathrm{p}=0.39)$.

Taken together, though memantine showed a mild anxiolytic effect in the open filed test, none of the medications was able to augment cognitive performance in APP/PS1 mice when given at low dosages either alone or in combination. Experiments were conducted in two batches including both genders. Consistency of data was confirmed by two-way ANOVA as no significant differences could be observed between mice from different batches or gender. 


\section{Discussion}

\subsection{Interregional differences outweigh age and disease}

In this study I aimed to predict and validate a novel therapeutic strategy based on a longitudinal transcriptional and epigenetic characterization of a mouse model for Alzheimer's disease. The APP/PS1 mouse model B6-Tg(Thy1-APPswe; Thy1-PS1 L166P) (Radde et al., 2006) is frequently used in current science for studying the cognitive decline as well as the immune response upon amyloid deposition (Bittner et al., 2012; Jay et al., 2015). The hippocampus and isocortex are brain areas strikingly affected by amyloid deposition both in human AD patients at severe stages and in the given AD mouse model (Braak and Braak, 1991; Radde et al., 2006). While the neuropathology in APP/PS1 mice emerges from the hippocampus, isocortical regions like the ACC are only affected in progressed disease stages.

Due to the time-dependent spread of amyloid deposition and the resulting effect on neurodegeneration, it is important to specifically study distinct brain regions. Even though the hippocampal sub-regions are most likely affected by the transgene at roughly the same time and severity in APP/PS1 mice, their functions in cognition are quite diverse (Kandel, 2013; Radde et al., 2006). I thus speculated that their transcriptional profiles themselves are diverse as well. Using Illumina RNA sequencing, I was in fact able to show that the transcriptional profiles of the two anatomically neighboring hippocampal areas DG and CA1 are as distinct as either one of those is compared to the ACC and that approximately 20 to $30 \%$ of the entire transcriptome is differential in distinct brain regions. Furthermore, I found that these differences completely outweigh any effects of the transgene or aging in those mice as the only observed clusters could be related to brain regions. As a general phenomenon throughout the different approaches of data analysis, the DG always seemed to be the most variable brain region and, interestingly, was only mildly affected at an age of 4 months compared to the ACC or CA1. Notably, the DG is one of the few sites in the adult murine brain were neurogenesis happens throughout maturity (Cameron et al., 1993). Adult neurogenesis does not only play a physiological role in cognitive processes like pattern recognition, it is also likely to be an important compensatory mechanism for neurodegeneration 
and was even shown to be elevated in some mouse models for AD (Deng et al., 2009; Winner et al., 2011). This might explain the increased variability and the relatively mild progression of the pathology in the DG of APP/PS1 transgenic mice.

In addition to the region-specific transcriptomic diversity, data on DNA methylation revealed striking differences between cell types as well compared to only mild differences between the genotypes. This is in line with findings from Zeisel et al. (2015) who was even able to split subcell-types in cortical and hippocampal tissue simply based on their transcription profiles. Taken together, these data clearly indicate the need for brain region-specific but also cell type-specific molecular approaches whenever applicable.

\subsection{Compensatory effects in young APP/PS1 mice temporarily delay cognitive decline}

Preliminary data from our lab revealed that juvenile APP/PS1 mice show no cognitive impairment yet. Here, I showed that these findings on mice behavior do very nicely coincide with transcriptomic data, as the differences in gene expression found between 1.5 months old wildtype and transgenic mice were only marginal. Interestingly, pathway analysis revealed neuronal plasticity related functions when considering mildly deregulated genes, i.e. "Axon guidance" and "Calcium Signaling". Furthermore, the genes involved in those pathways were mainly regulated in a gain-of-function manner in transgenic mice, indicated by an upregulation of the NMDA-receptor domains GRIN1 and GRIN2D, MAPK3, and UNC5A or a downregulation of CD38. This might indicate a compensatory effect of gene expression in transgenic mice counteracting the incipient amyloid pathology. For instance, a reduction of CD38 was shown to drive NGFinduced neuronal differentiation and neurite outgrowth, thereby promoting survival of newborn neurons, while UNC5A was shown to promote axon guidance acting as a receptor for netrin1 (Hedgecock et al., 1990; Serafini et al., 1996; Yue et al., 2009).

Radde et al. described the APP/PS1 mouse model in 2006 and showed that though the mice did not show any amyloid plaques yet at 1 months of age, quite a substantial number of plaques were formed at an age of 2 month. The chosen age of 1.5 months is thus nicely reflecting a 
stage where the mice are cognitively fine but neurodegeneration is just starting to occur and thus compensatory effects are likely to still maintain homeostasis at least partially. Another example of these compensatory effects besides the promotion of neuronal differentiation is the clearance of amyloid deposits by microglia. While microglia are in principle able to scavenge the brain from neurotoxic amyloid, they become dysfunctional with progressing disease, loosing their phagocytotic ability and even promoting neurodegeneration (Jones et al., 2014; Khoury et al., 1996; Meda et al., 1995). This dysfunction of microglia, however, is just one of the immune response related effects that are dominating the entire gene expression profiles in APP/PS1 mice at later time points.

\subsection{Cognitive decline in APP/PS1 mice is accompanied by a progressive induction of immune response}

In early 2015, Gjoneska et al. published a similar approach for characterizing AD in another mouse model for the disease, the CK-p25 mice. They found a two-directional effect of the pathology on gene expression: A downregulation of genes that can be linked to cognitive decline and a broad upregulation of immune response related gene expression. However, they did not consider the fold change of differential gene expression for their analysis. In the present study, I analyzed gene expression profiles of APP/PS1 transgenic mice in a longitudinal approach differentially for mildly and severely affected genes. I was able to confirm the findings made for CK-p25 mice in APP/PS1 mice as well. Furthermore, I found that downregulation of gene expression linked to cognitive decline is rather mild and that the gene expression profile per se is masked by a strong induction of immune response related genes. Notably, most of the changes in gene expression upon early amyloid pathology worsen during age progression. The induction of immune response could be linked to a well defined core-set of TFs including proteins belonging to the ETS, GATA, IRF, KLF, NfKB and STAT families.

Interestingly, TFs were previously shown to interact quite frequently with each other thereby modulating their regulatory effect. One of the main TFs of the ETS family not only responsible for deregulated gene expression but also deregulated in its expression itself is SPI1. In central nervous tissue, SPI1 is specific for microglia and a TF essential for cell viability and phagocytotic 
activity (Smith et al., 2013). Along with other core-set TFs like IRF8, it is crucial for the synthesis of new microglia from erythromyeloid precursor cells (Kierdorf et al., 2013). IRF8 also functions in the regulation of neuronal cell death, as reinstatement of IRF8 expression in neurons can prevent apoptosis upon ischemia, and interacts with TFs of the STAT family (Xiang et al., 2014). Even though STAT activity is often linked with immune response, apoptosis and cancer pathways, knowledge on its role in healthy cognition is increasing in the recent years (Thomas et al., 2015). STAT signaling is not only crucial for the synthesis of new astrocytes, activation of the JAK/STAT-pathway upon interleukin-6 and interleukin-15 signaling was shown to be involved in adult neurogenesis as well (Bauer, 2009; Bonni et al., 1997; Gómez-Nicola et al., 2011). Opposing effects of the different STAT proteins were reported on neuronal functioning. While STAT3 activity was implicated in NMDA-receptor dependent long-term depression, spatial memory formation is negatively regulated by STAT1 (Hsu et al., 2013; Nicolas et al., 2012). STAT3 was also shown to interact with NFkB, whose activation is partially driving cell death in the CNS (Liu et al., 2011; Zhang et al., 2005). GATA proteins like GATA2 and GATA3 are highly implicated in the synthesis of new blood cells (Ku et al., 2012; Rodrigues et al., 2012). Interestingly, they were also shown to trigger pre- and post-natal neurogenesis and do thus function in neural development and cognition (Nardelli et al., 1999; Zhao et al., 2008). Altogether, the identified core-set TFs do not only drive the immune response in APP/PS1 mice, they are also a link between the induced immune response and an impaired neuronal functioning.

Only a fraction of the described core-set TFs was also found to be differentially expressed themselves, however, the activity of all described TFs is tightly regulated by posttranslational mechanisms, for instance acetylation and phosphorylation (Cowley and Graves, 2000; Lamonica et al., 2006; Zhuang, 2013). Interestingly, these posttranslational modifications are often regulated by proteins involved in epigenetic modifications and TFs were shown to form complexes with these proteins, including histone acetyltransferases and HDACs (Feng et al., 2010; Icardi et al., 2012; Ray et al., 2013; Suzuki et al., 2003). Though the majority of these proteins were not affected in their gene expression in APP/PS1 mice, histone acetylation is affected in those mice and other mouse models for $\mathrm{AD}$ and targeting histone acetylation is considered a promising therapeutic approach for counteracting the disease (Benito et al., 2015; Francis et al., 2009; Xu et al., 2011). The frequently described beneficial effect of different HDAC-inhibitors on preventing cognitive 
decline or reinstating memory function is thus likely not solely dependent on epigenetic modifications but also based on the interaction between HDACs and TFs (Gaub et al., 2010; Peleg et al., 2010; Ricobaraza et al., 2009). In summary, the core-set TFs do not only act in immune response but also in neuronal functioning and homeostasis. This might indicate a role in compensatory mechanisms, trying to reinstate a healthy cellular environment and coping with neurodegeneration, driven by amyloid deposits. Furthermore, reinstating a healthy regulation of gene expression via these TFs might be a promising approach to ameliorate the AD pathology and could be achieved by directly targeting the TFs or enzymes involved in posttranslational modifications.

\subsection{Alternative splicing of only few genes in APP/PS1 mice might facilitate amyloid deposition and cognitive decline}

The two major neuropathologies in AD patients are the amyloid plaques and tau NFT. The formation of these plaques and tangles does not only depend on the frequency of posttranslational processing, i.e. cleavage of APP resulting into $A \beta_{42}$, but also on differential composition of APP and tau themselves resulting from differential splicing (Belyaev et al., 2010; Kar et al., 2005; Rockenstein et al., 1995). Here, I analyzed alternate splicing in APP/PS1 mice to discover other genes whose splice variants might contribute to the progression of the disease and to study the effect of amyloidosis on the splicosome. Interestingly, the analysis on amyloidosis driven changes in alternative splicing showed that the splicosome is not dramatically effected by amyloid deposition. Notably, the splicosome within the ACC of young mice was severely affected by the transgene. However, this effect is unlikely caused by amyloid deposits as the ACC is not affected by amyloidosis at that stage (Radde et al., 2006). These findings again highlight the need for region specific analysis and suggest that alternative splicing is rather functioning in neuronal development and aging than neurodegeneration.

Even though, the effect of amyloidosis on alternative splicing was mild, I was able to identify some significant splice variants of APP processing and recognizing enzymes like PS1, Ctsd (Cathepsin D) and Trem2 (Triggering Receptor Expressed on Myeloid cells 2) as well as APP itself in the tested brain areas of 4 and 8 months old mice. Notably, tau was not found to be differentially spliced, indicating the specific effect of alternate splicing triggered by the transgenic APP 
and PS1. Differential exon usage in the PS1-gene was already described in 1997, though not much is known about potential differences in the activity of PS1 splice variants (Rogaev et al., 1997). An intronic mutation of PS1 can lead to an insertion of a single codon resulting in an induction of $A \beta_{42}$ synthesis suggesting a similar effect of differential splicing as well (De Jonghe et al., 1999). CTSD can cleave APP at its $\gamma$-cleavage site, thereby generating the neurotoxic $A \beta_{42}$ (Sadik et al., 1999). However, nothing is known so far about differential splicing of Ctsd and its consequences.

TREM2 forms a transmembrane complex with TYROBP (TYRO protein tyrosine kinase Binding Protein) and was proposed to function in the recognition of amyloid and subsequent activation of microglial phagocytosis (Frank et al., 2008; Hickman and El Khoury, 2014). Mutations in the Trem2 gene were shown to drastically increase the risk for AD (Guerreiro et al., 2013; Jonsson et al., 2013). Furthermore, different protein variants of TREM 2 were identified that function as risk factors for $\mathrm{AD}$ as well, though it remained unknown whether these variants are the result of alternate splicing or posttranslational modifications (Jin et al., 2014). One of these variants lacks a transmembrane domain, forming a soluble isoform that might inhibit the function of the membrane-bound TREM2 (Piccio et al., 2008; Schmid et al., 2002). Interestingly, this soluble form of TREM2 was found to be increased in the cerebrospinal fluid of patients suffering from central nervous diseases like multiple sclerosis but also $\mathrm{AD}$, though its actual role in promoting diseases remains unknown (Heslegrave et al., 2016).

Two more genes were identified to be differentially spliced throughout disease progression involved in neuronal functioning rather than amyloid processing, namely Ntrk2 (Neurotrophic Tyrosine Kinase, receptor type 2) and Thy1 (Thymocyte differentiation antigen 1). Ntrk2, also known as tyrosine receptor kinase B, recognizes BDNF (Brain Derived Neurotrophic Factor) and as such is involved in promoting differentiation and survival of newborn neurons (Bartkowska et al., 2007; Bergami et al., 2009). In regard to AD, Ntrk2 was shown to be indirectly influenced by $A \beta$ through calpain leading to cleavage of the receptor and thus impairment of BDNF signaling (Jerónimo-Santos et al., 2015). Furthermore, different splice variants of Ntrk2 were shown to influence APP-intracellular domain levels and might thus also influence the production of neurotoxic A $\beta$ species (Ansaloni et al., 2011). Thy1, also known as CD90 (Cluster of Differentiation 90), was first described in 1964 and found to be highly expressed in T-cells and nervous tissue 
(Reif and Allen, 1964). Due to its high expression in neuronal tissue, the Thy1 promoter is widely used for generating transgenic mice (Dana et al., 2014; Feng et al., 2000; Radde et al., 2006). The APP/PS1 mouse line used in the presented study is only one example for these mouse models. Despite the high expression of Thy1 in nervous tissue and its effect on long-term potentiation in the DG and axon regeneration, knowledge about its role in central nervous diseases is quite sparse (Deininger et al., 2003; Nosten-Bertrand et al., 1996). Even though no splice variants of Thy1 are described yet, data from differential splicing might indicate splice variants of Thy1 which might then contribute to the cognitive decline or promote compensatory mechanisms in APP/PS1 mice. Taken together, I was able to highlight a specific effect of the APP/PS1 transgene on differential splicing of genes with isoforms and splice variants that were already previously linked to cognitive decline or immune response in regard to neurodegenerative diseases. Furthermore, the presented data imply the need for further studies on splice variants of Thy1 and Ctsd in regard to $\mathrm{AD}$.

A stronger effect on differential exon usage was, however, identified during aging in both wildtype and transgenic mice. In fact, previously published data from our lab already suggested a striking effect of aging on alternate splicing linked to cognitive decline rather than neuroinflammation (Stilling et al., 2014). I was thus again able to validate the previously described linkage between aging and differential splicing in a different experimental approach (Mazin et al., 2014; Tollervey et al., 2011). Taken together these data indicate a naturally occurring differential exon usage in neuronal development following endogenous requirements on splice variants that is specifically impacted by amyloid deposition in a small number of genes. This disruption of differential splicing might then additionally promote neurodegeneration and thus cognitive decline in APP/PS1 mice.

\subsection{DNA methylation acts via long-distance interactions in AP- P/PS1 mice}

Data from RNAseq revealed a strong effect of the transgene on gene expression. Changes in gene expression can result from different molecular processes. These processes include the binding of TFs and subsequent initiation of gene expression as well as epigenetic mechanisms. These 
epigenetic mechanisms include DNA methylation and histone modification. Here, I analyzed DNA methylation profiles in an approach similar to differential gene expression analysis with MeDIPseq and identified some significant changes in all three tested brain regions upon amyloid pathology. Interestingly, no consistent effect could be detected throughout aging. DNA methylation is in general associated with gene silencing and in fact I found levels of DNA methylation decreased in genes with high gene expression (Newell-Price et al., 2000). Furthermore, the majority of sequenced bins was found in distal intergenic regions, thus regions were transcription should not occur. Interestingly however, quite a substantial fraction of significant differences was detected in those distal intergenic regions as well, indicating a rather unspecific effect of the transgene on DNA methylation. Concordantly, no functional pathways were significantly overrepresented by genes where DNA methylation was shown to be deregulated. These findings match data on human AD patients, where DNA methylation changes in the frontal cortex were shown to cover a broad variety of cellular processes rather than specific pathways (Bakulski et al., 2012). This unspecific effect on the genome might resemble a global deterioration of transcriptional regulation and might facilitate disease progression.

Other labs have already investigated the effect of $A \beta$ on DNA methylation levels in different $A D$ mouse models or cell culture (Isaacs et al., 2013; Sanchez-Mut et al., 2013) and found a correlation between DNA methylation and gene expression only in a small set of genes. Here, I show on a genome-wide scale that, even though a small number of genes shows a reduced gene expression and an increase in DNA methylation or vice versa, DNA methylation and gene expression can not be correlated on a larger scale. Additionally, I presented the first data ever showing cell-type specific DNA methylation in an AD mouse model giving a better understanding on the differential effect of amyloid pathology on neuronal and non-neuronal cells. Notably, Isaacs et al. (2013) found a link between DNA methylation and gene expression mainly in genes involved in cellfate determination. This is in line with my findings, indicating that DNA methylation is rather involved in differentiating cell-types and only plays a secondary role in responding to endogenous or exogenous stimuli. These stimuli might be disturbances in homeostasis, for instance caused by amyloid plaques, but also external stimuli causing neuronal activity. Recent data from our lab showed the effect of fear conditioning on DNA methylation in the hippocampus 
and ACC (Halder et al., 2015). During memory acquisition, the number of differentially methylated genes was comparable to that found in APP/PS1 mice and did not reach the dimension of cell-type related differences. Furthermore, Halder et al. also showed that the majority of differential DNA methylation does not occur within genes but in intergenic regions, rather suggesting long distance interactions between $\mathrm{CpG}$ sites and genes presumably regulated through the transcriptional repressor CTCF (Wang et al., 2012).

Notably, CTCF was shown to mediate interactions between chromatin and the nuclear lamina and thereby chromatin architecture (Handoko et al., 2011). Mutations in CTCF can lead to disturbances in higher order chromatin structures and were found to cause cognitive dysfunctions (Watson et al., 2014). Additionally, gene expression of APP is regulated via CTCF related pathways as well (Burton et al., 2002). The chromatin-lamina interactions are also regulated by the chromatin state giving a link between disruptions in the epigenetic machinery and subsequent changes of gene expression in distal chromosomal regions or even different chromosomes (Harr et al., 2015). Here I showed that potential impact of DNA methylation on gene expression upon amyloidosis are rather in the dimensions of interchromosomal interactions than those within defined interaction domains of up to 10 million base pairs and do thus depend on changes of the chromosomal architecture (Dixon et al., 2012). Additionally, I claim that direct effects of DNA methylation on gene expression are rather involved in determining cell fate and suggest further studies on the cell type specific mediation of chromatin organization, also mediated via CTCF as it was only insignificantly studied yet (Hou et al., 2010).

\subsection{The impact of amyloidosis on H3K4me3 remains elusive}

Apart from the rather global and cellular function-independent primary effect of DNA methylation, it can still be considered a mark for gene silencing and I was able to find DNA methylation reduced in active genes. To further identify the epigenetic mechanisms underlying the massive changes in gene expression, I also aimed to investigate an active epigenetic mark. Trimethylation of H3K4 is in general implicated with transcriptionally active genes (Ruthenburg et al., 2007). Via ChIP-sequencing on H3K4me3, I could confirm the previously described correlation between high levels of H3K4me3 and a high rate of gene expression previously. Surprisingly 
though, the only significantly differential H3K4me3 peaks were found within the Thy1 gene for most age groups in both the ACC and DG of transgenic mice. The only exception were nonneuronal cells from the DG in 4 month old mice. Interestingly, this very age group and brain region revealed only few changes in gene expression, primarily upregulated while H3K4me3 was globally downregulated. In regard to $A D, A \beta$ was previously shown to increase the level of H3K4me3 in two hormone-genes specifically (CORT and SST) (Rubio et al., 2012). These changes were not identified in this study. Furthermore, studies on the Ck-p25 AD mouse model revealed massive changes of H3K4me3 (Gjoneska et al., 2015). The data from the described ChIPseq experiments are thus partially contradictory to previously described data on H3K4me3 in regard to AD. Even though a decent quality of ChIP-sequencing was validated for each sample individually and the general linkage between active gene expression and H3K4me3 could be confirmed, I can not rule out an artificially introduced bias and absence of significant difference between wildtype and transgenic mice resulting from the small technical sample size $(n=2)$ and relatively high variance, thus weak statistical power.

\subsection{Drug screening and treatment of APP/PS1 mice}

Compared to gene expression, changes in splicing and DNA methylation were rather unrelated to cellular functions and inconsistent throughout disease progression. I thus decided to base the screening for novel therapeutic drugs for AD on gene expression data. Rather than interfering with the activity of some differentially expressed genes, I claim that it is more promising to target core modulators of gene expression. These core modulators include TFs and thus I screened for overrepresented TFs and defined a core-set of these responsible for a large fraction of differentially expressed genes. Targeting these proteins might normalize gene expression of a larger portion of deregulated genes. In fact, a variety of the defined core-set TFs is involved in amyloid deposit induced signaling and some of them were already studied in regard of AD themselves. Phosphorylation of STAT3, for instance, is reduced in AD patients and different mouse models for AD upon amyloid deposition (Chiba et al., 2009). Healthy levels of phosphorylated STAT3 could be reinstated using the PI-3K inhibitor LY294002, though was not yet thoroughly studied in regard to AD (Fang et al., 2006). STAT1 was shown to mediate the memory-impairing effect of A $\beta$ and 
can be inhibited by fludarabine, a therapeutic drug for treating different types of cancer (Frank et al., 1999; Hsu et al., 2013). Some phytoestrogens are able to suppress both phospho-STAT1 and IRF mediated inflammation and might serve as potential drugs for AD as well (Jantaratnotai et al., 2013).

After identifying those promising drug targets, I additionally performed a goal-directed drug screening based on all the differentially expressed genes and identified a variety of drug candidates that might reinstate a healthy gene expression. The most abundantly identified drugs included SAHA, trichostatin A, acacetin, parthenolide, pirinixid acid, piperlongumine, lovastatin, sirolimus, valproic acid and kaempferol. Notably, even though not among the top 10 drugs, memantine was identified by the drug pair seeker as well and is a commonly used drug in $\mathrm{AD}$ patients. Some of these top 10 drugs can be linked to AD due to their mode of action or were even tested already in mouse models for AD. For instance, the flavone-derivate acacetin was shown to suppress neuroinflammation through $\mathrm{NF}_{\mathrm{KB}}$ and $\mathrm{NO}$ signaling and might thus be suitable for targeting the inflammatory response in AD (Ha et al., 2012; Nathan et al., 2005). Parthenolide was shown to prevent the formation of amyloid-like fibers and also shows anti-inflammatory functions but was like acacetin not tested in regard to AD yet (Mathema et al., 2012; Romero et al., 2013). In contrast, sirolimus was already successfully tested in a mouse model for AD, leading to a reduction of $A \beta$ and tau pathology (Caccamo et al., 2010). Interestingly, three of the top10 identified drugs (SAHA, trichostatin A and valproic acid) are widely used HDAC-inhibitors that were recently tested in regard to AD (Benito et al., 2015; Kazantsev and Thompson, 2008; Noh and Seo, 2014; Wang et al., 2012). SAHA was found to elevate cognitive performance in aged wildtype mice by reinstating histone acetylation and a subsequent pre-clinical study in our lab confirmed a beneficial effect of this particular HDAC-inhibitor on cognition in APP/PS1 mice, linked with a reduction in neuro-inflammation (Benito et al., 2015; Peleg et al., 2010). A clinical study on vorinostat in $\mathrm{AD}$ patients is most likely going to follow, confirming the procedure described in this work as promising and valid for discovering novel therapeutic approaches.

Since SAHA was recently confirmed as a promising treatment for AD, I hypothesized that a combinatory treatment of APP/PS1 mice using SAHA along with a second drug might reduce the necessary dosage of both drugs and widen their therapeutic effects. As SAHA is supposedly mainly acting on reinstating gene expression through histone acetylation and was shown to have 
anti-inflammatory potential (Benito et al., 2015), I chose memantine for the combinatory treatment of APP/PS1 transgenic mice. Memantine is commonly used in AD patients and facilitates synaptic plasticity and thus cognitive function by antagonizing NMDA receptors and reinstating glutamatergic signaling (Barnes et al., 1996). Unfortunately, I was not able to identify any beneficial effect of the combinatory treatment in regard to anxiety-like and exploratory behavior or spatial and contextual memory acquisition and retrieval. The dosages of the chosen drugs were designed to represent a reduced dosage analogue to those used for human patients and compared to the standard dosages used in mice to enable identification of an augmented effect. Corresponding dosages for memantine were previously shown not do bear the full therapeutic potential though a similar dosage was able to reinstate memory functions in another mouse model for AD (Liu et al., 2014; Minkeviciene et al., 2004; Saab et al., 2011). Since both drugs were previously successfully studied in APP/PS1 transgenic mice, it is likely that they were not able to augment the therapeutic potential of the respective other drug simply due to the intentionally chosen reduced dosage and the relatively mild expressed cognitive decline at the given age of 5 to 6 months. Further experiments are needed to shed some more light on a potential advantage of combinatory treatments of SAHA and memantine. 


\section{Conclusions}

Alzheimer's disease is characterized by a progressive cognitive decline coinciding with the formation of amyloid plaques and tau fibrils in the human brain. It is the most common form of dementia and the number of people suffering from dementia worldwide was recently estimated to 46.8 million people, our knowledge on the molecular basics of $\mathrm{AD}$ and its development is rather little. Even though many different therapeutic drugs were tested in pre-clinical trials and some drugs are commonly used for treating AD patients, no cure is available yet. Unfortunately, the possibilities to study the disease in human patients is limited and mainly rely on post-mortem tissue. Thus, mouse models specifically reflecting different aspects of the disease were generated during the recent years.

In this study, I used an APP/PS1 transgenic mouse line to study the specific effect of amyloid pathology on gene expression, DNA methylation and histone methylation, thereby identifying core modulators of disease progression and new potential therapeutic strategies for counteracting AD. I was able to show that amyloid deposits on gene expression are independent from the respective brain region, though the transcription profiles of these brain regions are strikingly diverse. This global deregulation of gene expression can be linked to a small set of TFs that might serve as target proteins for therapeutic drugs. Gene expression is mainly driven by immune response related processes while deregulation of genes that function in neuronal processes is rather mild. Interestingly though, the identified significant TFs bridge the induction of immune response upon amyloid deposition with a loss of neuronal function leading to cognitive decline. The deregulation of genes and corresponding functional pathways becomes more severe with increasing age and thus follows the progressive cognitive decline observed in APP/PS1 mice. The wildtype-like cognitive performance of young transgenic mice can be explained by compensatory mechanisms counteracting the effect of neurotoxic $A \beta$. These compensatory mechanisms become overwhelmed while $A \beta$ plaques spread, leading to pathological aging.

In contrast to the strong effect of the immune response on gene expression, splicing does almost exclusively affect neuronal functioning and is rather affected by aging than amyloidosis. Nonetheless, I was able to detect differentially spliced genes with splice variants previously 
shown to promote the disease and identified two genes where alternative splicing was not yet related to neurodegenerative diseases, namely Thy1 and Ctsd. The deregulation of gene expression observed in APP/PS1 mice might partially be driven my a global disruption of the epigenetic landscape concerning DNA methylation. This disruption, is likely to cause a deterioration in higher order chromatin structures and might thereby impact gene expression rather indirectly. In contrast, I claim that gene-directed effects of DNA methylation leading to the silencing of genes is primarily involved in encoding cell-fate.

By using the DrugPairSeeker on RNAseq data, I identified a number of drugs potentially able to reinstate healthy gene expression in APP/PS1 transgenic mice through diverse mechanisms. Some of these drugs were already successfully tested in animal models for AD and our group recently confirmed a beneficial effect of the HDAC-inhibitor SAHA on cognition and neuroinflammation in regard to AD. Here, I studied the therapeutic potential of a combinatory treatment of SAHA along with the NMDA-receptor antagonist memantine, a commonly used drug for AD patients. I hypothesized, that a combinatory treatment might broaden the medications' efficacy and enables the use of mild dosages of the individual drugs thereby limiting adverse side effects. Unfortunately, a beneficial effect on memory functioning of either drug individually or in combination was not confirmed for APP/PS1 mice, presumably due to the reduced dosages and mild cognitive impairment observed in mice of the chosen age.

Altogether, I identified a set of core-modulators of differential gene expression and novel alternative splicing in the Thy1 and Ctsd genes that might drive disease progression. Furthermore, I hypothesize that the severe disruptions of gene expression are at least partially driven by deterioration of the chromatin architecture resulting from deregulated epigenetic mechanisms. Further experiments on DNA-DNA interactions via "chromosome confirmation capture" approaches and DNA-protein interactions, i.e. regarding CTCF, are necessary to test this hypothesis. Based on RNAseq data, I predicted a set of therapeutic drugs able to counteract amyloidosis in AD patients and illustrated the need for further studies concerning the combinatory usage of these drugs for enhancing the treatment of human patients. Based on previous studies regarding the identified drugs, I conclude that the applied procedure in this work is a suitable and valid approach for discovering novel therapeutic drugs for counteracting AD and other human diseases. 


\section{Supplemental figures and tables}
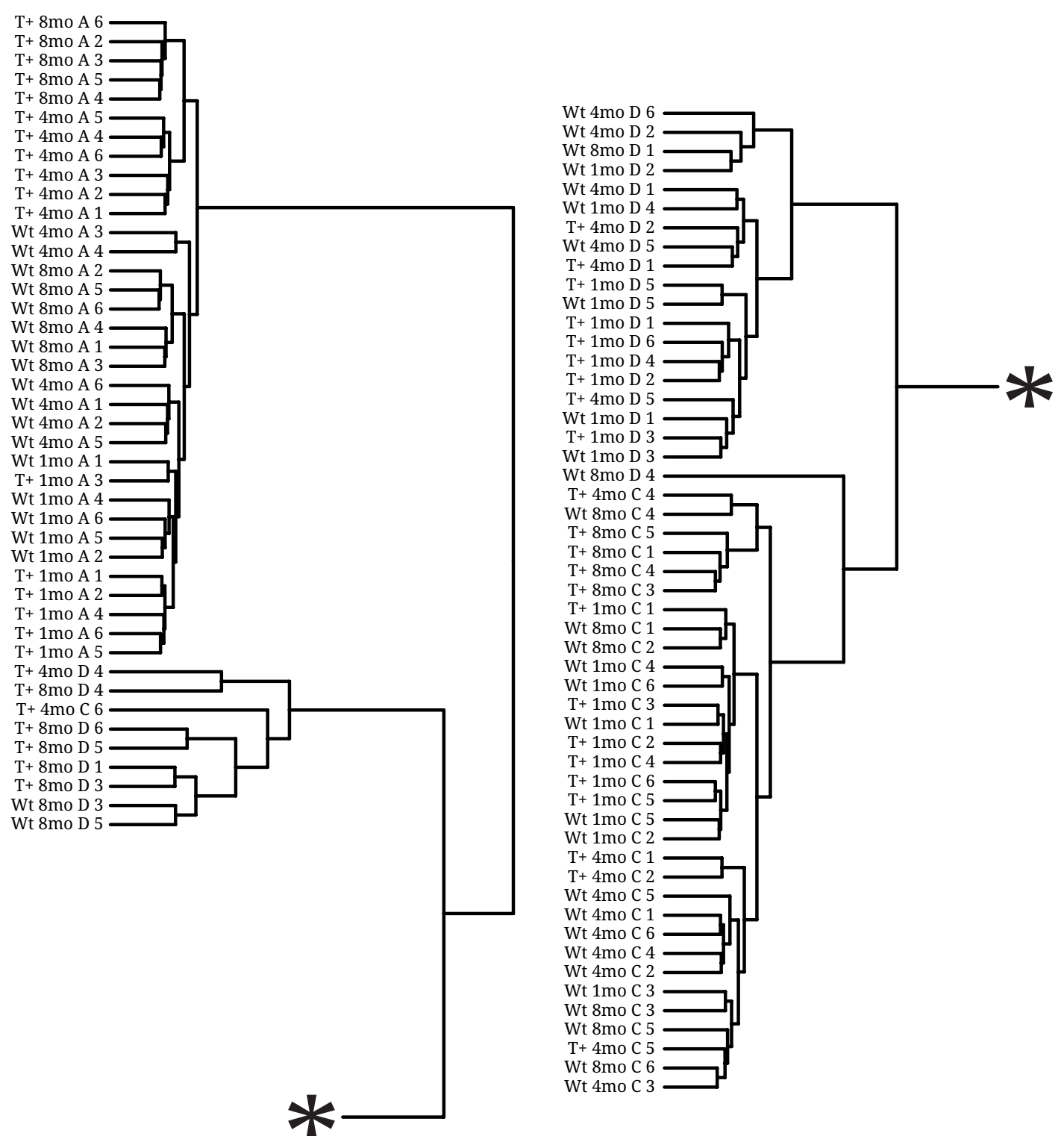

Figure 7.1 - Detailed dendrogram for eucledian distances of RNA samples:

Detailed view on the dendrogram and sample IDs shown above and below the distance heatmap depicted in figure 4.1A (see p.57). Wt: wildtype; T+: transgenic. A: ACC; C: CA1; D: DG. 
$\overleftrightarrow{\tilde{o}^{\prime}}$ Table 7.1 - KEGG pathways enriched by interregional differences common in different age groups

\begin{tabular}{|l|l|l|}
\hline ACC vs CA1 & ACC vs DG & CA vs DG \\
\hline Arrhythmogenic right ventricular cardiomyopathy & Adherens junction & Arrhythmogenic right ventricular cardiomyopathy \\
\hline Axon guidance & Arrhythmogenic right ventricular cardiomyopathy & Axon guidance \\
\hline Basal cell carcinoma & Axon guidance & Basal cell carcinoma \\
\hline Calcium signaling pathway & Calcium signaling pathway & Calcium signaling pathway \\
\hline Dilated cardiomyopathy & Colorectal cancer & Dilated cardiomyopathy \\
\hline ECM-receptor interaction & Dilated cardiomyopathy & ECM-receptor interaction \\
\hline Focal adhesion & ECM-receptor interaction & ErbB signaling pathway \\
\hline Hypertrophic cardiomyopathy (HCM) & ErbB signaling pathway & Focal adhesion \\
\hline MAPK signaling pathway & Fc gamma R-mediated phagocytosis & Gap junction \\
\hline Melanogenesis & Focal adhesion & GnRH signaling pathway \\
\hline Neuroactive ligand-receptor interaction & Gap junction & Hedgehog signaling pathway \\
\hline Pathways in cancer & GnRH signaling pathway & Heparan sulfate biosynthesis \\
\hline Ribosome & Hypertrophic cardiomyopathy (HCM) & Hypertrophic cardiomyopathy (HCM) \\
\hline Wnt signaling pathway & Inositol phosphate metabolism & Long-term potentiation \\
\hline & MAPK signaling pathway & MAPK signaling pathway \\
\hline & Neuroactive ligand-receptor interaction & Melanogenesis \\
\hline & p53 signaling pathway & Neuroactive ligand-receptor interaction \\
\hline & Pathways in cancer & p53 signaling pathway \\
\hline & Phosphatidylinositol signaling system & Pathways in cancer \\
\hline & Regulation of actin cytoskeleton & Phosphatidylinositol signaling system \\
\hline & Renal cell carcinoma & Regulation of actin cytoskeleton \\
\hline & Ribosome & TGF-beta signaling pathway \\
\hline & Type II diabetes mellitus & Vascular smooth muscle contraction \\
\hline & Vascular smooth muscle contraction & \\
\hline & Wnt signaling pathway & \\
\hline & & \\
\hline
\end{tabular}

Axon guidance

Dilated cardiomyopathy

Fc gamma R-mediated phagocytosis

rophic cardiomyopathy ( $\mathrm{HCM})$

MAPK signaling pathway

Neuroactive ligand-receptor interaction

Phosphatidylinositol signaling system

Renal cell carcinom

Vascular smooth muscle co

gnaling pathwa 
Table 7.2 - Canonical pathways for upregulated genes in 1.5 months old APP/PS1 mice: ACC

\begin{tabular}{|l|c|}
\hline Pathway & -lg(pvalue) \\
\hline TR/RXR Activation & 3.01 \\
\hline Germ Cell-Sertoli Cell Junction Signaling & 2.85 \\
\hline Ephrin Receptor Signaling & 2.62 \\
\hline Unfolded protein response & 2.23 \\
\hline Superpathway of Cholesterol Biosynthesis & 2.22 \\
\hline
\end{tabular}

Table 7.3 - Top 10 canonical pathways for upregulated genes in 4 months old APP/PS1 mice: ACC

\begin{tabular}{|l|c|}
\hline Pathway & -lg(pvalue) \\
\hline TREM1 Signaling & 16.9 \\
\hline Dendritic Cell Maturation & 15.5 \\
\hline Superpathway of Cholesterol Biosynthesis & 14.8 \\
\hline Antigen Presentation Pathway & 14.1 \\
\hline T Helper Cell Differentiation & 13.8 \\
\hline Role of Pattern Recognition Receptors in Recognition of Bacteria and Viruses & 12.7 \\
\hline phagosome formation & 12.1 \\
\hline Altered T Cell and B Cell Signaling in Rheumatoid Arthritis & 12 \\
\hline Granulocyte Adhesion and Diapedesis & 10.4 \\
\hline Leukocyte Extravasation Signaling & 10.1 \\
\hline
\end{tabular}

Table 7.4 - Top 10 canonical pathways for upregulated genes in 8 months old APP/PS1 mice: ACC

\begin{tabular}{|l|c|}
\hline Pathway & -lg(pvalue) \\
\hline TREM1 Signaling & 18 \\
\hline Dendritic Cell Maturation & 14.2 \\
\hline Role of Pattern Recognition Receptors in Recognition of Bacteria and Viruses & 14.1 \\
\hline EIF2 Signaling & 14.1 \\
\hline Superpathway of Cholesterol Biosynthesis & 13.9 \\
\hline Altered T Cell and B Cell Signaling in Rheumatoid Arthritis & 13.7 \\
\hline T Helper Cell Differentiation & 13 \\
\hline phagosome formation & 11.6 \\
\hline Role of Macrophages, Fibroblasts and Endothelial Cells in Rheumatoid Arthritis & 11.4 \\
\hline Antigen Presentation Pathway & 11 \\
\hline
\end{tabular}


Table 7.5 - Canonical pathways for downregulated genes in 1.5 months old APP/PS1 mice: ACC

\begin{tabular}{|l|c|}
\hline Pathway & -lg(pvalue) \\
\hline Glycine Degradation (Creatine Biosynthesis) & 2.28 \\
\hline eNOS Signaling & 2.21 \\
\hline
\end{tabular}

Table 7.6 - Top 10 canonical pathways for downregulated genes in 4 months old APP/PS1 mice: ACC

\begin{tabular}{|l|c|}
\hline Pathway & -lg(pvalue) \\
\hline Superpathway of Inositol Phosphate Compounds & 3.94 \\
\hline Integrin Signaling & 3.83 \\
\hline Axonal Guidance Signaling & 3.65 \\
\hline G-Protein Coupled Receptor Signaling & 3.6 \\
\hline Ephrin A Signaling & 3.6 \\
\hline Semaphorin Signaling in Neurons & 3.39 \\
\hline CCR3 Signaling in Eosinophils & 3.31 \\
\hline ErbB Signaling & 3.26 \\
\hline D-myo-inositol (1,4,5)-trisphosphate Degradation & 2.93 \\
\hline Neuropathic Pain Signaling In Dorsal Horn Neurons & 2.91 \\
\hline
\end{tabular}

Table 7.7 - Top 10 canonical pathways for downregulated genes in 8 months old APP/PS1 mice: ACC

\begin{tabular}{|l|c|}
\hline Pathway & -lg(pvalue) \\
\hline G-Protein Coupled Receptor Signaling & 10.9 \\
\hline Synaptic Long Term Potentiation & 8.82 \\
\hline cAMP-mediated signaling & 8.79 \\
\hline Dopamine-DARPP32 Feedback in cAMP Signaling & 8.73 \\
\hline Neuropathic Pain Signaling In Dorsal Horn Neurons & 8.14 \\
\hline CREB Signaling in Neurons & 7.97 \\
\hline Axonal Guidance Signaling & 7.59 \\
\hline Breast Cancer Regulation by Stathmin1 & 7.14 \\
\hline Molecular Mechanisms of Cancer & 6.78 \\
\hline GNRH Signaling & 6.77 \\
\hline
\end{tabular}


Table 7.8 - Canonical pathways for upregulated genes in 1.5 months old APP/PS1 mice: CA1

\begin{tabular}{|l|c|}
\hline Pathway & -lg(pvalue) \\
\hline Amyloid Processing & 2.76 \\
\hline Integrin Signaling & 2.74 \\
\hline Regulation of Cellular Mechanics by Calpain Protease & 2.66 \\
\hline FAK Signaling & 2.3 \\
\hline Apoptosis Signaling & 2.28 \\
\hline Tryptophan Degradation to 2-amino-3-carboxymuconate Semialdehyde & 2.01 \\
\hline
\end{tabular}

Table 7.9 - Top 10 canonical pathways for upregulated genes in 4 months old APP/PS1 mice: CA1

\begin{tabular}{|l|c|}
\hline Pathway & -lg(pvalue) \\
\hline Superpathway of Cholesterol Biosynthesis & 12.2 \\
\hline Antigen Presentation Pathway & 12.1 \\
\hline Dendritic Cell Maturation & 11.3 \\
\hline Altered T Cell and B Cell Signaling in Rheumatoid Arthritis & 10.5 \\
\hline Communication between Innate and Adaptive Immune Cells & 10.4 \\
\hline TREM1 Signaling & 10.4 \\
\hline T Helper Cell Differentiation & 9.57 \\
\hline Complement System & 9.26 \\
\hline Cholesterol Biosynthesis I & 7.37 \\
\hline Cholesterol Biosynthesis II (via 24,25-dihydrolanosterol) & 7.37 \\
\hline
\end{tabular}

Table 7.10 - Top 10 canonical pathways for upregulated genes in 8 months old APP/PS1 mice: CA1

\begin{tabular}{|l|c|}
\hline Pathway & -lg(pvalue) \\
\hline TREM1 Signaling & 20.3 \\
\hline Role of Pattern Recognition Receptors in Recognition of Bacteria and Viruses & 19.1 \\
\hline Dendritic Cell Maturation & 18.6 \\
\hline Superpathway of Cholesterol Biosynthesis & 16.7 \\
\hline Altered T Cell and B Cell Signaling in Rheumatoid Arthritis & 15.7 \\
\hline phagosome formation & 15.4 \\
\hline Role of Macrophages, Fibroblasts and Endothelial Cells in Rheumatoid Arthritis & 13.9 \\
\hline T Helper Cell Differentiation & 13.8 \\
\hline Type I Diabetes Mellitus Signaling & 12.7 \\
\hline Antigen Presentation Pathway & 12.3 \\
\hline
\end{tabular}


Table 7.11 - Top 10 canonical pathways for downregulated genes in 8 months old APP/PS1 mice: CA1

\begin{tabular}{|l|c|}
\hline Pathway & -lg(pvalue) \\
\hline Dopamine-DARPP32 Feedback in cAMP Signaling & 11.2 \\
\hline Breast Cancer Regulation by Stathmin1 & 10.5 \\
\hline Role of NFAT in Cardiac Hypertrophy & 8.66 \\
\hline GABA Receptor Signaling & 7.92 \\
\hline Axonal Guidance Signaling & 6.73 \\
\hline Melatonin Signaling & 6.03 \\
\hline nNOS Signaling in Neurons & 5.8 \\
\hline Thrombin Signaling & 5.77 \\
\hline Protein Kinase A Signaling & 5.73 \\
\hline HIPPO signaling & 5.53 \\
\hline
\end{tabular}

Table 7.12 - Canonical pathways for upregulated genes in 1.5 months old APP/PS1 mice: DG

\begin{tabular}{|l|c|}
\hline Pathway & -lg(pvalue) \\
\hline LXR/RXR Activation & 3.97 \\
\hline PCP pathway & 2.96 \\
\hline Uracil Degradation II (Reductive) & 2.51 \\
\hline Thymine Degradation & 2.51 \\
\hline FXR/RXR Activation & 2.37 \\
\hline Acute Phase Response Signaling & 2.12 \\
\hline Granulocyte Adhesion and Diapedesis & 2.09 \\
\hline
\end{tabular}

Table 7.13 - Top 10 canonical pathways for upregulated genes in 4 months old APP/PS1 mice: DG

\begin{tabular}{|l|c|}
\hline Pathway & -lg(pvalue) \\
\hline Granulocyte Adhesion and Diapedesis & 6.83 \\
\hline Dendritic Cell Maturation & 5.52 \\
\hline TREM1 Signaling & 4.58 \\
\hline Agranulocyte Adhesion and Diapedesis & 4.15 \\
\hline Complement System & 4.08 \\
\hline phagosome formation & 3.95 \\
\hline Caveolar-mediated Endocytosis Signaling & 3.24 \\
\hline Production of Nitric Oxide and Reactive Oxygen Species in Macrophages & 3.12 \\
\hline Communication between Innate and Adaptive Immune Cells & 2.95 \\
\hline Coagulation System & 2.54 \\
\hline
\end{tabular}


Table 7.14 - Top 10 canonical pathways for upregulated genes in 8 months old APP/PS1 mice: DG

\begin{tabular}{|l|c|}
\hline Pathway & -lg(pvalue) \\
\hline Altered T Cell and B Cell Signaling in Rheumatoid Arthritis & 14.4 \\
\hline Dendritic Cell Maturation & 14 \\
\hline TREM1 Signaling & 12.4 \\
\hline Communication between Innate and Adaptive Immune Cells & 12.1 \\
\hline Antigen Presentation Pathway & 11.5 \\
\hline phagosome formation & 11.4 \\
\hline T Helper Cell Differentiation & 10.7 \\
\hline CD28 Signaling in T Helper Cells & 9.84 \\
\hline Complement System & 8.92 \\
\hline Fcy Receptor-mediated Phagocytosis in Macrophages and Monocytes & 8.82 \\
\hline
\end{tabular}

Table 7.15 - Canonical pathways for downregulated genes in 1.5 months old APP/PS1 mice: DG

\begin{tabular}{|l|c|}
\hline Pathway & -lg(pvalue) \\
\hline Parkinson's Signaling & 2 \\
\hline
\end{tabular}

Table 7.16 - Top 10 canonical pathways for downregulated genes in 8 months old APP/PS1 mice: DG

\begin{tabular}{|l|c|}
\hline Pathway & -lg(pvalue) \\
\hline Axonal Guidance Signaling & 6.12 \\
\hline Glutamate Dependent Acid Resistance & 3.52 \\
\hline G-Protein Coupled Receptor Signaling & 3.22 \\
\hline TR/RXR Activation & 3.14 \\
\hline Xenobiotic Metabolism Signaling & 2.99 \\
\hline GABA Receptor Signaling & 2.96 \\
\hline p70S6K Signaling & 2.93 \\
\hline Nitric Oxide Signaling in the Cardiovascular System & 2.73 \\
\hline GNRH Signaling & 2.71 \\
\hline Breast Cancer Regulation by Stathmin1 & 2.7 \\
\hline
\end{tabular}


Table 7.17 - Core-set of transcription factors including ETS, GATA, IRF, KLF, NFKB and STAT

\begin{tabular}{|c|c|}
\hline Protein family & Transcription factor \\
\hline \multirow{12}{*}{ ETS } & Ehf \\
\hline & Elf1 \\
\hline & Elf5 \\
\hline & Elk1 \\
\hline & Elk4 \\
\hline & Erg \\
\hline & Ets1 \\
\hline & Fev \\
\hline & Fli \\
\hline & GABPA \\
\hline & Spi1 \\
\hline & SpiB \\
\hline \multirow{4}{*}{ GATA } & Gata1 \\
\hline & Gata2 \\
\hline & Gata3 \\
\hline & Gata4 \\
\hline
\end{tabular}

\begin{tabular}{|c|c|}
\hline Protein family & Transcription factor \\
\hline \multirow{2}{*}{ IRF } & Irf1 \\
\hline & Irf2 \\
\hline \multirow{3}{*}{ KLF } & Klf1 \\
\hline & Klf4 \\
\hline & Klf5 \\
\hline \multirow{3}{*}{$\mathrm{NF} \kappa \mathrm{B}$} & Nf-kB1 \\
\hline & Rel \\
\hline & RelA \\
\hline \multirow{7}{*}{ STAT } & Stat1 \\
\hline & Stat2 \\
\hline & Stat3 \\
\hline & Stat4 \\
\hline & Stat5A \\
\hline & Stat5B \\
\hline & Stat6B \\
\hline
\end{tabular}

Table 7.18 - Hot-spots of gene expression in APP/PS1 mice $4 \mathrm{mo}$ ACC

8 mo ACC

\begin{tabular}{|l|l|l|l|}
\hline domain & chrom. & start & stop \\
\hline chr17-16 & 17 & 33943055 & 34503055 \\
\hline
\end{tabular}

8mo DG

\begin{tabular}{|l|l|l|l|}
\hline domain & chrom. & start & stop \\
\hline chr1-100 & 1 & 170869869 & 172349869 \\
\hline chr4-60 & 4 & 136044085 & 137644085 \\
\hline chr17-16 & 17 & 33943055 & 34503055 \\
\hline
\end{tabular}

\begin{tabular}{|l|l|l|l|}
\hline domain & chrom. & start & stop \\
\hline chr1-100 & 1 & 170869869 & 172349869 \\
\hline chr7-50 & 7 & 102451486 & 105851486 \\
\hline chr7-66 & 7 & 127976486 & 128736486 \\
\hline chr7-74 & 7 & 141494101 & 143814095 \\
\hline chr11-21 & 11 & 46426498 & 49586498 \\
\hline chr13-10 & 13 & 21588131 & 23748131 \\
\hline chr17-16 & 17 & 33943055 & 34503055 \\
\hline
\end{tabular}




\section{List of abbreviations}

\begin{tabular}{|c|c|}
\hline$A \beta$ & amyloid $\beta$ \\
\hline A.D. & Auguste Deter \\
\hline ac & acetylation \\
\hline ACC & anterior cingulate cortex \\
\hline $\mathrm{AD}$ & Alzheimer's disease \\
\hline APP & amyloid precursor protein \\
\hline BACE1 & $\beta$-site APP cleaving enzyme 1 \\
\hline CA & cornu ammonis \\
\hline ChIP & Chromatin immunoprecipitation \\
\hline CNS & central nervous system \\
\hline Ctsd & Cathepsin D \\
\hline DG & dentate gyrus \\
\hline DNMT & DNA methyltransferases \\
\hline FACS & fluorescence-activated cell sorting \\
\hline HDAC & histone deacetylases \\
\hline KEGG & Kyoto Enzyclopedia of Genes and Genomes \\
\hline LTM & long-term memory \\
\hline me & methylation \\
\hline MeDIP & Methylated DNA immunoprecipitation \\
\hline NFT & neurofibrillary tangles \\
\hline NGS & Next-generation sequencing \\
\hline PAM & partition around medoid \\
\hline PCA & Principal component analysis \\
\hline PS/PSEN & presenilin \\
\hline RT & room temperature \\
\hline SAHA & suberoylanilide hydroxamic acid \\
\hline STM & short-term memory \\
\hline TFs & transcription factors \\
\hline Thy1 & Thymocyte differentiation antigen 1 \\
\hline Trem2 & Triggering Receptor Expressed on Myeloid cells 2 \\
\hline TYROBP & TYRO protein tyrosine kinase Binding Protein \\
\hline
\end{tabular}




\section{Bibliography}

Abel, T., Lattal, K. M., 2001. Molecular mechanisms of memory acquisition, consolidation and retrieval. Current opinion in neurobiology 11 (2), 180-187.

Adwan, L., Zawia, N. H., Apr. 2013. Epigenetics: A novel therapeutic approach for the treatment of Alzheimer's disease. Pharmacology \& therapeutics.

Agis-Balboa, R. C., Arcos-Diaz, D., Wittnam, J., Govindarajan, N., Blom, K., Burkhardt, S., Haladyniak, U., Agbemenyah, H. Y., Zovoilis, A., Salinas-Riester, G., et al., 2011. A hippocampal insulin-growth factor 2 pathway regulates the extinction of fear memories. The EMBO Journal.

Aimone, J., Wiles, J., Gage, F., 2009. Computational influence of adult neurogenesis on memory encoding. Neuron 61 (2), 187-202.

Allis, C. D., Jenuwein, T., Reinberg, D., 2007. Epigenetics. Cold Spring Harbor Laboratory Press, Cold Spring Harbor, N.Y.

Alonso, A. C., Grundke-Iqbal, I., Iqbal, K., Jul. 1996. Alzheimer's disease hyperphosphorylated tau sequesters normal tau into tangles of filaments and disassembles microtubules. Nature Medicine 2 (7), 783-787.

ALZFORUM, Jan. 2016. ALZFORUM Database: Mutations.

Alzheimer, A., Stelzmann, R. A., Schnitzlein, H. N., Murtagh, F. R., 1995. An English translation of Alzheimer's 1907 paper, ”Uber eine eigenartige Erkankung der Hirnrinde”. Clinical Anatomy (New York, N.Y.) 8 (6), 429-431.

Anders, S., Huber, W., 2010. Differential expression analysis for sequence count data. Genome Biology 11, R106.

Anders, S., Reyes, A., Huber, W., 2012. Detecting differential usage of exons from rna-seq data. Genome Research 22, 4025.

Andersen, P., Morris, R., Amaral, D., Bliss, T., O’Keefe, J., 2007. The Hippocampus book. Oxford University Press, Oxford.

Andrews, S., 2010. FastQC A Quality Control tool for High Throughput Sequence Data.

Ansaloni, S., Leung, B. P., Sebastian, N. P., Samudralwar, R., Gadaleta, M., Saunders, A. J., 2011. TrkB Isoforms Differentially Affect AICD Production through Their Intracellular Functional Domains. International Journal of Alzheimer's Disease 2011, 1-11. 
Araki, W., Kitaguchi, N., Tokushima, Y., Ishii, K., Aratake, H., Shimohama, S., Nakamura, S., Kimura, J., Nov. 1991. Trophic effect of beta-amyloid precursor protein on cerebral cortical neurons in culture. Biochemical and Biophysical Research Communications 181 (1), 265-271.

Association, A., Mar. 2015. 2015 Alzheimer’s disease facts and figures. Alzheimer's \& Dementia 11 (3), 332384.

Avery, O. T., Macleod, C. M., McCarty, M., Feb. 1944. Studies on the chemical nature of the substance inducing transformation of pneumococcal types. Inductions of transformation by a desoxyribonucleic acid fraction isolated from pneumococcus type III. The Journal of Experimental Medicine 79 (2), 137-158.

Bailey, C. H., Bartsch, D., Kandel, E. R., Nov. 1996. Toward a molecular definition of long-term memory storage. Proceedings of the National Academy of Sciences of the United States of America 93 (24), 1344513452.

Bakulski, K. M., Dolinoy, D. C., Sartor, M. A., Paulson, H. L., Konen, J. R., Lieberman, A. P., Albin, R. L., Hu, H., Rozek, L. S., 2012. Genome-wide DNA methylation differences between late-onset Alzheimer's disease and cognitively normal controls in human frontal cortex. Journal of Alzheimer's Disease 29 (3), 571-588.

Bard, F., Cannon, C., Barbour, R., Burke, R. L., Games, D., Grajeda, H., Guido, T., Hu, K., Huang, J., JohnsonWood, K., Khan, K., Kholodenko, D., Lee, M., Lieberburg, I., Motter, R., Nguyen, M., Soriano, F., Vasquez, N., Weiss, K., Welch, B., Seubert, P., Schenk, D., Yednock, T., Aug. 2000. Peripherally administered antibodies against amyloid beta-peptide enter the central nervous system and reduce pathology in a mouse model of Alzheimer disease. Nature Medicine 6 (8), 916-919.

Barnes, C. A., Danysz, W., Parsons, C. G., 1996. Effects of the Uncompetitive NMDA Receptor Antagonist Memantine on Hippocampal Long-term Potentiation, Short-term Exploratory Modulation and Spatial Memory in Awake, Freely Moving Rats. European Journal of Neuroscience 8 (3), 565-571.

Barnes, D. E., Yaffe, K., Sep. 2011. The projected effect of risk factor reduction on Alzheimer's disease prevalence. The Lancet Neurology 10 (9), 819-828.

Bartkowska, K., Paquin, A., Gauthier, A. S., Kaplan, D. R., Miller, F. D., Dec. 2007. Trk signaling regulates neural precursor cell proliferation and differentiation during cortical development. Development 134 (24), $4369-4380$.

Bartlett, F. C., 1995. Remembering: a study in experimental and social psychology. Cambridge University Press, Cambridge ; New York. 
Bartus, R., Dean, R., Beer, B., Lippa, A., Jul. 1982. The cholinergic hypothesis of geriatric memory dysfunction. Science 217 (4558), 408-414.

Battaglia, F. P., Benchenane, K., Sirota, A., Pennartz, C. M., Wiener, S. I., Jun. 2011. The hippocampus: hub of brain network communication for memory. Trends in Cognitive Sciences.

Bauer, S., Feb. 2009. Cytokine Control of Adult Neural Stem Cells: Chronic versus Acute Exposure. Annals of the New York Academy of Sciences 1153 (1), 48-56.

Beach, T. G., Monsell, S. E., Phillips, L. E., Kukull, W., Apr. 2012. Accuracy of the clinical diagnosis of Alzheimer disease at National Institute on Aging Alzheimer Disease Centers, 2005-2010. Journal of Neuropathology and Experimental Neurology 71 (4), 266-273.

Bednar, J., Horowitz, R. A., Grigoryev, S. A., Carruthers, L. M., Hansen, J. C., Koster, A. J., Woodcock, C. L., Nov. 1998. Nucleosomes, linker DNA, and linker histone form a unique structural motif that directs the higher-order folding and compaction of chromatin. Proceedings of the National Academy of Sciences 95 (24), 14173-14178.

Belyaev, N. D., Kellett, K. A. B., Beckett, C., Makova, N. Z., Revett, T. J., Nalivaeva, N. N., Hooper, N. M., Turner, A. J., Dec. 2010. The Transcriptionally Active Amyloid Precursor Protein (APP) Intracellular Domain Is Preferentially Produced from the 695 Isoform of APP in a -Secretase-dependent Pathway. Journal of Biological Chemistry 285 (53), 41443-41454.

Benito, E., Urbanke, H., Ramachandran, B., Barth, J., Halder, R., Awasthi, A., Jain, G., Capece, V., Burkhardt, S., Navarro-Sala, M., Nagarajan, S., Schütz, A.-L., Johnsen, S. A., Bonn, S., Lührmann, R., Dean, C., Fischer, A., Sep. 2015. HDAC inhibitor-dependent transcriptome and memory reinstatement in cognitive decline models. Journal of Clinical Investigation 125 (9), 3572-3584.

Bergami, M., Berninger, B., Canossa, M., 2009. Conditional deletion of TrkB alters adult hippocampal neurogenesis and anxiety-related behavior. Communicative \& Integrative Biology 2 (1), 14-16.

Berkyurek, A. C., Suetake, I., Arita, K., Takeshita, K., Nakagawa, A., Shirakawa, M., Tajima, S., Jan. 2014. The DNA Methyltransferase Dnmt1 Directly Interacts with the SET and RING Finger-associated (SRA) Domain of the Multifunctional Protein Uhrf1 to Facilitate Accession of the Catalytic Center to Hemi-methylated DNA. Journal of Biological Chemistry 289 (1), 379-386.

Berman, B. P., Weisenberger, D. J., Aman, J. F., Hinoue, T., Ramjan, Z., Liu, Y., Noushmehr, H., Lange, C. P. E., van Dijk, C. M., Tollenaar, R. A. E. M., Van Den Berg, D., Laird, P. W., Nov. 2011. Regions of focal DNA hypermethylation and long-range hypomethylation in colorectal cancer coincide with nuclear lamina-associated domains. Nature Genetics 44 (1), 40-46. 
Bhasin, M., Reinherz, E. L., Reche, P. A., Feb. 2006. Recognition and classification of histones using support vector machine. Journal of computational biology: a journal of computational molecular cell biology 13 (1), 102-112.

Bittner, T., Burgold, S., Dorostkar, M. M., Fuhrmann, M., Wegenast-Braun, B. M., Schmidt, B., Kretzschmar, H., Herms, J., Dec. 2012. Amyloid plaque formation precedes dendritic spine loss. Acta Neuropathologica 124 (6), 797-807.

Bonn, S., Zinzen, R. P., Perez-Gonzalez, A., Riddell, A., Gavin, A.-C., Furlong, E. E. M., Apr. 2012. Cell type-specific chromatin immunoprecipitation from multicellular complex samples using BiTS-ChIP. Nature Protocols 7 (5), 978-994.

Bonni, A., Sun, Y., Nadal-Vicens, M., Bhatt, A., Frank, D. A., Rozovsky, I., Stahl, N., Yancopoulos, G. D., Greenberg, M. E., 1997. Regulation of gliogenesis in the central nervous system by the JAK-STAT signaling pathway. Science 278 (5337), 477-483.

Braak, H., Braak, E., 1991. Neuropathological stageing of Alzheimer-related changes. Acta neuropathologica 82 (4), 239-259.

Brandt, R., Lee, G., Feb. 1993. Functional organization of microtubule-associated protein tau. Identification of regions which affect microtubule growth, nucleation, and bundle formation in vitro. The Journal of Biological Chemistry 268 (5), 3414-3419.

Brantley, D. M., Chen, C.-L., Muraoka, R. S., Bushdid, P. B., Bradberry, J. L., Kittrell, F., Medina, D., Matrisian, L. M., Kerr, L. D., Yull, F. E., May 2001. Nuclear Factor- B (NF- B) Regulates Proliferation and Branching in Mouse Mammary Epithelium. Molecular Biology of the Cell 12 (5), 1445-1455.

Bredesen, D. E., 2015. Metabolic profiling distinguishes three subtypes of Alzheimer's disease. Aging (Albany NY) 7 (8), 595.

Bruen, P. D., McGeown, W. J., Shanks, M. F., Venneri, A., Aug. 2008. Neuroanatomical correlates of neuropsychiatric symptoms in Alzheimer's disease. Brain 131 (9), 2455-2463.

Bubenzer-Busch, S., Herpertz-Dahlmann, B., Kuzmanovic, B., Gaber, T. J., Helmbold, K., Ullisch, M. G., Baurmann, D., Eickhoff, S. B., Fink, G. R., Zepf, F. D., Aug. 2015. Neural correlates of reactive aggression in children with attention-deficit/hyperactivity disorder and comorbid disruptive behaviour disorders. Acta Psychiatrica Scandinavica, n/a-n/a. 
Burns, A., Rossor, M., Hecker, J., Gauthier, S., Petit, H., Möller, H. J., Rogers, S. L., Friedhoff, L. T., Jun. 1999. The effects of donepezil in Alzheimer's disease - results from a multinational trial. Dementia and Geriatric Cognitive Disorders 10 (3), 237-244.

Burton, T., Liang, B., Dibrov, A., Amara, F., Jul. 2002. Transforming growth factor- $\beta$-induced transcription of the Alzheimer $\beta$-amyloid precursor protein gene involves interaction between the CTCF-complex and Smads. Biochemical and Biophysical Research Communications 295 (3), 713-723.

Bush, G., Luu, P., Posner, M. I., 2000. Cognitive and emotional influences in anterior cingulate cortex. Trends in cognitive sciences 4 (6), 215-222.

Butovsky, O., Talpalar, A. E., Ben-Yaakov, K., Schwartz, M., Jul. 2005. Activation of microglia by aggregated $\beta$-amyloid or lipopolysaccharide impairs MHC-II expression and renders them cytotoxic whereas IFN- $\gamma$ and IL-4 render them protective. Molecular and Cellular Neuroscience 29 (3), 381-393.

Butterfield, D. A., Swomley, A. M., Sultana, R., Sep. 2013. Amyloid $\beta$-Peptide (1-42)-Induced Oxidative Stress in Alzheimer Disease: Importance in Disease Pathogenesis and Progression. Antioxidants \& Redox Signaling 19 (8), 823-835.

Caccamo, A., Majumder, S., Richardson, A., Strong, R., Oddo, S., Apr. 2010. Molecular interplay between mammalian target of rapamycin (mTOR), amyloid-beta, and Tau: effects on cognitive impairments. The Journal of Biological Chemistry 285 (17), 13107-13120.

Cameron, H. A., Woolley, C. S., McEwen, B. S., Gould, E., Sep. 1993. Differentiation of newly born neurons and glia in the dentate gyrus of the adult rat. Neuroscience 56 (2), 337-344.

Cao, L., Jiao, X., Zuzga, D. S., Liu, Y., Fong, D. M., Young, D., During, M. J., Jul. 2004. VEGF links hippocampal activity with neurogenesis, learning and memory. Nature Genetics 36 (8), 827-835.

Carlson, M., 2015. org.Mm.eg.db: Genome wide annotation for Mouse. R package version 3.2.3.

Carlson, M., Maintainer, B. P., 2015. TxDb.Mmusculus.UCSC.mm10.ensGene: Annotation package for TxDb object(s). R package version 3.2.2.

Carola, V., D’Olimpio, F., Brunamonti, E., Mangia, F., Renzi, P., 2002. Evaluation of the elevated plus-maze and open-field tests for the assessment of anxiety-related behaviour in inbred mice. Behavioural brain research 134 (1), 49-57.

Carter, C. S., Botvinick, M. M., Cohen, J. D., 1999. The contribution of the anterior cingulate cortex to executive processes in cognition. Reviews in the Neurosciences 10 (1), 49-57. 
Castel, S. E., Martienssen, R. A., Jan. 2013. RNA interference in the nucleus: roles for small RNAs in transcription, epigenetics and beyond. Nature Reviews Genetics 14 (2), 100-112.

Castellucci, V. F., Kandel, E. R., Dec. 1974. A quantal analysis of the synaptic depression underlying habituation of the gill-withdrawal reflex in Aplysia. Proceedings of the National Academy of Sciences of the United States of America 71 (12), 5004-5008.

Cataldo, J. K., Prochaska, J. J., Glantz, S. A., 2010. Cigarette smoking is a risk factor for Alzheimer's disease: an analysis controlling for tobacco industry affiliation. Journal of Alzheimer's disease: JAD 19 (2), 465.

Cazalis, F., Babikian, T., Giza, C., Copeland, S., Hovda, D., Asarnow, R. F., 2011. Pivotal Role of Anterior Cingulate Cortex in Working Memory after Traumatic Brain Injury in Youth. Frontiers in Neurology 1.

Cervoni, N., Bhattacharya, S., Szyf, M., 1999. DNA demethylase is a processive enzyme. Journal of Biological Chemistry 274 (13), 8363-8366.

Chapel, H. M., Esiri, M. M., Wilcock, G. K., 1984. Immunoglobulin and other proteins in the cerebrospinal fluid of patients with Alzheimer's disease. Journal of clinical pathology 37 (6), 697-699.

Chen, S., Townsend, K., Goldberg, T. E., Davies, P., Conejero-Goldberg, C., 2010. MAPT isoforms: differential transcriptional profiles related to 3r and 4r splice variants. Journal of Alzheimer's Disease 22 (4), 1313.

Chiba, T., Yamada, M., Sasabe, J., Terashita, K., Shimoda, M., Matsuoka, M., Aiso, S., 2009. Amyloid- $\beta$ causes memory impairment by disturbing the JAK2/STAT3 axis in hippocampal neurons. Molecular psychiatry $14(2), 206-222$.

Clamp, M., Fry, B., Kamal, M., Xie, X., Cuff, J., Lin, M. F., Kellis, M., Lindblad-Toh, K., Lander, E. S., 2007. Distinguishing protein-coding and noncoding genes in the human genome. Proceedings of the National Academy of Sciences 104 (49), 19428-19433.

Cleary, J. P., Walsh, D. M., Hofmeister, J. J., Shankar, G. M., Kuskowski, M. A., Selkoe, D. J., Ashe, K. H., Jan. 2005. Natural oligomers of the amyloid- $\beta$ protein specifically disrupt cognitive function. Nature Neuroscience 8 (1), 79-84.

Cole, C. S., Richards, K. C., Jan. 2005. Sleep and Cognition in People with Alzheimer's Disease. Issues in Mental Health Nursing 26 (7), 687-698.

Cong, L., Jia, J., Qin, W., Ren, Y., Sun, Y., Sep. 2014. Genome-wide analysis of DNA methylation in an APP/PS1 mouse model of Alzheimer's disease. Acta Neurologica Belgica 114 (3), 195-206. 
Coppieters, N., Dieriks, B. V., Lill, C., Faull, R. L., Curtis, M. A., Dragunow, M., Jun. 2014. Global changes in DNA methylation and hydroxymethylation in Alzheimer's disease human brain. Neurobiology of Aging 35 (6), 1334-1344.

Cowan, N., 2008. What are the differences between long-term, short-term, and working memory? Progress in brain research $169,323-338$.

Cowan, N., Chen, Z., Rouder, J. N., Sep. 2004. Constant capacity in an immediate serial-recall task: a logical sequel to Miller (1956). Psychological science 15 (9), 634-640.

Cowley, D. O., Graves, B. J., Feb. 2000. Phosphorylation represses Ets-1 DNA binding by reinforcing autoinhibition. Genes \& Development 14 (3), 366-376.

Cruz, J. C., Tseng, H.-C., Goldman, J. A., Shih, H., Tsai, L.-H., Oct. 2003. Aberrant Cdk5 Activation by p25 Triggers Pathological Events Leading to Neurodegeneration and Neurofibrillary Tangles. Neuron 40 (3), 471-483.

Cummings, J. L., Morstorf, T., Zhong, K., 2014. Alzheimer’s disease drug-development pipeline: few candidates, frequent failures. Alzheimers Res Ther 6 (4), 37.

Dana, H., Chen, T.-W., Hu, A., Shields, B. C., Guo, C., Looger, L. L., Kim, D. S., Svoboda, K., Sep. 2014. Thy1GCaMP6 Transgenic Mice for Neuronal Population Imaging In Vivo. PLoS ONE 9 (9), e108697.

Dawkins, E., Small, D. H., Jun. 2014. Insights into the physiological function of the $\beta$-amyloid precursor protein: beyond Alzheimer's disease. Journal of Neurochemistry 129 (5), 756-769.

Day, J. J., Sweatt, J. D., Nov. 2010. DNA methylation and memory formation. Nature Neuroscience 13 (11), 1319-1323.

De Jonghe, C., MarcCruts, Rogaeva, E. A., Tysoe, C., Singleton, A., Vanderstichele, H., Meschino, W., Dermaut, B., Vanderhoeven, I., Backhovens, H., Vanmechelen, E., Morris, C. M., Hardy, J., Rubinsztein, D. C., St George-Hyslop, P. H., Van Broeckhoven, C., Aug. 1999. Aberrant Splicing in the Presenilin-1 Intron 4 Mutation Causes Presenile Alzheimer's Disease by Increased A 42 Secretion. Human Molecular Genetics 8 (8), 1529-1540.

De Strooper, B., Iwatsubo, T., Wolfe, M. S., Jan. 2012. Presenilins and -Secretase: Structure, Function, and Role in Alzheimer Disease. Cold Spring Harbor Perspectives in Medicine 2 (1), a006304-a006304.

Deary, I. J., Corley, J., Gow, A. J., Harris, S. E., Houlihan, L. M., Marioni, R. E., Penke, L., Rafnsson, S. B., Starr, J. M., Dec. 2009. Age-associated cognitive decline. British Medical Bulletin 92 (1), 135-152. 
Deininger, S.-O., Rajendran, L., Lottspeich, F., Przybylski, M., Illges, H., Stuermer, C. A., Reuter, A., Apr. 2003. Identification of teleost Thy-1 and association with the microdomain/lipid raft reggie proteins in regenerating CNS axons. Molecular and Cellular Neuroscience 22 (4), 544-554.

DeMattos, R. B., Bales, K. R., Cummins, D. J., Dodart, J. C., Paul, S. M., Holtzman, D. M., Jul. 2001. Peripheral anti-A beta antibody alters CNS and plasma A beta clearance and decreases brain A beta burden in a mouse model of Alzheimer's disease. Proceedings of the National Academy of Sciences of the United States of America 98 (15), 8850-8855.

Deng, J. C., Zeng, X., Newstead, M., Moore, T. A., Tsai, W. C., Thannickal, V. J., Standiford, T. J., Sep. 2004. STAT4 Is a Critical Mediator of Early Innate Immune Responses against Pulmonary Klebsiella Infection. The Journal of Immunology 173 (6), 4075-4083.

Deng, W., Saxe, M., Gallina, I., Gage, F., 2009. Adult-born hippocampal dentate granule cells undergoing maturation modulate learning and memory in the brain. The Journal of Neuroscience 29 (43), 1353213542.

Denslow, S. A., Wade, P. A., Aug. 2007. The human Mi-2/NuRD complex and gene regulation. Oncogene 26 (37), 5433-5438.

Dixon, J. R., Selvaraj, S., Yue, F., Kim, A., Li, Y., Shen, Y., Hu, M., Liu, J. S., Ren, B., Apr. 2012. Topological domains in mammalian genomes identified by analysis of chromatin interactions. Nature 485 (7398), 376-380.

Dobin, A., Davis, C. A., Schlesinger, F., Drenkow, J., Zaleski, C., Jha, S., Batut, P., Chaisson, M., Gingeras, T. R., 2013. STAR: ultrafast universal RNA-seq aligner. Bioinformatics 29 (1), 15-21.

Dover, G. J., Charache, S. H., Boyer, S. H., Talbot, C. C., Smith, K. D., 1983. 5-Azacytidine increases fetal hemoglobin production in a patient with sickle cell disease. Progress in Clinical and Biological Research $134,475-488$.

Dubois, B., Feldman, H. H., Jacova, C., DeKosky, S. T., Barberger-Gateau, P., Cummings, J., Delacourte, A., Galasko, D., Gauthier, S., Jicha, G., others, 2007. Research criteria for the diagnosis of Alzheimer's disease: revising the NINCDS-ADRDA criteria. The Lancet Neurology 6 (8), 734-746.

Dudai, Y., 2002. Memory from A to Z : keywords, concepts, and beyond. Oxford University Press, Oxford, UK.; New York.

El-Karim, E. A., Hagos, E. G., Ghaleb, A. M., Yu, B., Yang, V. W., 2013. Krüppel-like factor 4 regulates genetic stability in mouse embryonic fibroblasts. Molecular Cancer 12 (1), 89. 
Ende, G., Cackowski, S., Van Eijk, J., Sack, M., Demirakca, T., Kleindienst, N., Bohus, M., Sobanski, E., KrauseUtz, A., Schmahl, C., Jan. 2016. Impulsivity and Aggression in Female BPD and ADHD Patients: Association with ACC Glutamate and GABA Concentrations. Neuropsychopharmacology 41 (2), 410-418.

Fang, P., Hwa, V., Rosenfeld, R., May 2006. Interferon-gamma-induced dephosphorylation of STAT3 and apoptosis are dependent on the mTOR pathway. Experimental Cell Research 312 (8), 1229-1239.

Farovik, A., Dupont, L. M., Eichenbaum, H., Feb. 2010. Distinct roles for dorsal CA3 and CA1 in memory for sequential nonspatial events. Learning \& Memory 17 (1), 12-17.

Feng, D., Sangster-Guity, N., Stone, R., Korczeniewska, J., Mancl, M. E., Fitzgerald-Bocarsly, P., Barnes, B. J., Nov. 2010. Differential Requirement of Histone Acetylase and Deacetylase Activities for IRF5-Mediated Proinflammatory Cytokine Expression. The Journal of Immunology 185 (10), 6003-6012.

Feng, G., Mellor, R. H., Bernstein, M., Keller-Peck, C., Nguyen, Q. T., Wallace, M., Nerbonne, J. M., Lichtman, J. W., Sanes, J. R., 2000. Imaging neuronal subsets in transgenic mice expressing multiple spectral variants of GFP. Neuron 28 (1), 41-51.

Fischer, A., Jan. 2014. Targeting histone-modifications in Alzheimer's disease. What is the evidence that this is a promising therapeutic avenue? Neuropharmacology.

Fischer, A., Sananbenesi, F., Mungenast, A., Tsai, L.-H., Dec. 2010. Targeting the correct HDAC(s) to treat cognitive disorders. Trends in Pharmacological Sciences 31 (12), 605-617.

Fischer, A., Sananbenesi, F., Pang, P., Lu, B., Tsai, L., 2005. Opposing roles of transient and prolonged expression of p25 in synaptic plasticity and hippocampus-dependent memory. Neuron 48 (5), 825-838.

Forstl, H., Burns, A., Levy, R., Cairns, N., Jul. 1994. Neuropathological correlates of psychotic phenomena in confirmed Alzheimer's disease. The British Journal of Psychiatry 165 (1), 53-59.

Fox, L. M., William, C. M., Adamowicz, D. H., Pitstick, R., Carlson, G. A., Spires-Jones, T. L., Hyman, B. T., Jul. 2011. Soluble tau species, not neurofibrillary aggregates, disrupt neural system integration in a tau transgenic model. Journal of Neuropathology and Experimental Neurology 70 (7), 588-595.

Fraga, M. F., Esteller, M., Aug. 2007. Epigenetics and aging: the targets and the marks. Trends in Genetics 23 (8), 413-418.

Francis, Y. I., Fà, M., Ashraf, H., Zhang, H., Staniszewski, A., Latchman, D. S., Arancio, O., 2009. Dysregulation of histone acetylation in the APP/PS1 mouse model of Alzheimer's disease. Journal of Alzheimer's disease: JAD 18 (1), 131-139. 
Frank, D. A., Mahajan, S., Ritz, J., 1999. Fludarabine-induced immunosuppression is associated with inhibition of STAT1 signaling. Nature medicine 5 (4), 444-447.

Frank, S., Burbach, G. J., Bonin, M., Walter, M., Streit, W., Bechmann, I., Deller, T., Oct. 2008. TREM2 is upregulated in amyloid plaque-associated microglia in aged APP23 transgenic mice. Glia 56 (13), 14381447.

Frankland, P. W., Bontempi, B., Talton, L. E., Kaczmarek, L., Silva, A. J., 2004. The involvement of the anterior cingulate cortex in remote contextual fear memory. Science 304 (5672), 881-883.

Franklin, K. B. J., Paxinos, G., 1997. The mouse brain in stereotaxic coordinates. Academic Press, San Diego.

Gage, F. H., Feb. 2000. Mammalian Neural Stem Cells. Science 287 (5457), 1433-1438.

García-Matas, S., Vera, N. d., Adell, A., Planas, A. M., Cristòfol, R., Sanfeliu, C., others, 2010. In vitro and in vivo activation of astrocytes by amyloid- $\beta$ is potentiated by pro-oxidant agents. Journal of Alzheimer's Disease 20 (1), 229-245.

Gaub, P., Tedeschi, A., Puttagunta, R., Nguyen, T., Schmandke, A., Di Giovanni, S., 2010. HDAC inhibition promotes neuronal outgrowth and counteracts growth cone collapse through CBP/p300 and P/CAFdependent p53 acetylation. Cell Death \& Differentiation 17 (9), 1392-1408.

Gaume, X., Torres-Padilla, M.-E., Nov. 2015. Regulation of Reprogramming and Cellular Plasticity through Histone Exchange and Histone Variant Incorporation. Cold Spring Harbor Symposia on Quantitative Biology.

Ghosh, S., Echols, H., Dec. 1972. Purification and properties of D protein: a transcription factor of Escherichia coli. Proceedings of the National Academy of Sciences of the United States of America 69 (12), 3660-3664.

Giannakopoulos, P., Gold, G., Duc, M., Michel, J.-P., Hof, P. R., Bouras, C., 2000. Impaired Processing of Famous Faces in Alzheimer\&amp;rsquo;s Disease Is Related to Neurofibrillary Tangle Densities in the Prefrontal and Anterior Cingulate Cortex. Dementia and Geriatric Cognitive Disorders 11 (6), 336-341.

Ginsberg, S. D., Che, S., Counts, S. E., Mufson, E. J., Mar. 2006. Shift in the ratio of three-repeat tau and four-repeat tau mRNAs in individual cholinergic basal forebrain neurons in mild cognitive impairment and Alzheimer's disease: Tau expression in Alzheimer's disease. Journal of Neurochemistry 96 (5), 14011408. 
Gjoneska, E., Pfenning, A. R., Mathys, H., Quon, G., Kundaje, A., Tsai, L.-H., Kellis, M., Feb. 2015. Conserved epigenomic signals in mice and humans reveal immune basis of Alzheimer's disease. Nature 518 (7539), 365-369.

Goate, A., Chartier-Harlin, M.-C., Mullan, M., Brown, J., Crawford, F., Fidani, L., Giuffra, L., Haynes, A., Irving, N., James, L., Mant, R., Newton, P., Rooke, K., Roques, P., Talbot, C., Pericak-Vance, M., Roses, A., Williamson, R., Rossor, M., Owen, M., Hardy, J., Feb. 1991. Segregation of a missense mutation in the amyloid precursor protein gene with familial Alzheimer's disease. Nature 349 (6311), 704-706.

Goda, Y., Stevens, C. F., 1998. Readily releasable pool size changes associated with long term depression. Proceedings of the National Academy of Sciences 95 (3), 1283-1288.

Godyn, J., Jonczyk, J., Panek, D., Malawska, B., Feb. 2016. Therapeutic strategies for Alzheimer's disease in clinical trials. Pharmacological reports: PR 68 (1), 127-138.

Goedert, M., Spillantini, M. G., Jakes, R., Rutherford, D., Crowther, R. A., Oct. 1989. Multiple isoforms of human microtubule-associated protein tau: sequences and localization in neurofibrillary tangles of Alzheimer's disease. Neuron 3 (4), 519-526.

Goedert, M., Wischik, C. M., Crowther, R. A., Walker, J. E., Klug, A., Jun. 1988. Cloning and sequencing of the cDNA encoding a core protein of the paired helical filament of Alzheimer disease: identification as the microtubule-associated protein tau. Proceedings of the National Academy of Sciences of the United States of America 85 (11), 4051-4055.

Goenka, S., Kaplan, M. H., May 2011. Transcriptional regulation by STAT6. Immunologic Research 50 (1), 87-96.

Gold, M., Hurwitz, J., Anders, M., Apr. 1963. The enzymatic methylation of RNA and DNA. I. Biochemical and Biophysical Research Communications 11 (2), 107-114.

Goldgaber, D., Lerman, M. I., McBride, O. W., Saffiotti, U., Gajdusek, D. C., Feb. 1987. Characterization and chromosomal localization of a cDNA encoding brain amyloid of Alzheimer's disease. Science (New York, N.Y.) 235 (4791), 877-880.

Grundke-Iqbal, I., Iqbal, K., Quinlan, M., Tung, Y. C., Zaidi, M. S., Wisniewski, H. M., May 1986. Microtubuleassociated protein tau. A component of Alzheimer paired helical filaments. The Journal of Biological Chemistry 261 (13), 6084-6089. 
Gu, S. G., Pak, J., Guang, S., Maniar, J. M., Kennedy, S., Fire, A., Jan. 2012. Amplification of siRNA in Caenorhabditis elegans generates a transgenerational sequence-targeted histone H3 lysine 9 methylation footprint. Nature Genetics 44 (2), 157-164.

Guan, J.-S., Haggarty, S. J., Giacometti, E., Dannenberg, J.-H., Joseph, N., Gao, J., Nieland, T. J. F., Zhou, Y., Wang, X., Mazitschek, R., Bradner, J. E., DePinho, R. A., Jaenisch, R., Tsai, L.-H., 2009. HDAC2 negatively regulates memory formation and synaptic plasticity. Nature 459 (7243), 55-60.

Guan, Z., Giustetto, M., Lomvardas, S., Kim, J.-H., Miniaci, M. C., Schwartz, J. H., Thanos, D., Kandel, E. R., 2002. Integration of long-term-memory-related synaptic plasticity involves bidirectional regulation of gene expression and chromatin structure. Cell 111 (4), 483-493.

Guerreiro, R., Wojtas, A., Bras, J., Carrasquillo, M., Rogaeva, E., Majounie, E., Cruchaga, C., Sassi, C., Kauwe, J. S., Younkin, S., Hazrati, L., Collinge, J., Pocock, J., Lashley, T., Williams, J., Lambert, J.-C., Amouyel, P., Goate, A., Rademakers, R., Morgan, K., Powell, J., St. George-Hyslop, P., Singleton, A., Hardy, J., Jan. 2013. TREM2 Variants in Alzheimer’s Disease. New England Journal of Medicine 368 (2), 117-127.

Gómez-Isla, T., Hollister, R., West, H., Mui, S., Growdon, J. H., Petersen, R. C., Parisi, J. E., Hyman, B. T., 1997. Neuronal loss correlates with but exceeds neurofibrillary tangles in Alzheimer's disease. Annals of neurology 41 (1), 17-24.

Gómez-Nicola, D., Valle-Argos, B., Pallas-Bazarra, N., Nieto-Sampedro, M., 2011. Interleukin-15 regulates proliferation and self-renewal of adult neural stem cells. Molecular biology of the cell 22 (12), 1960-1970.

Götz, J., Ittner, L. M., Jul. 2008. Animal models of Alzheimer's disease and frontotemporal dementia. Nature Reviews Neuroscience 9 (7), 532-544.

Götz, J., Probst, A., Spillantini, M. G., Schäfer, T., Jakes, R., Bürki, K., Goedert, M., 1995. Somatodendritic localization and hyperphosphorylation of tau protein in transgenic mice expressing the longest human brain tau isoform. The EMBO journal 14 (7), 1304.

Ha, S. K., Moon, E., Lee, P., Ryu, J. H., Oh, M. S., Kim, S. Y., Jul. 2012. Acacetin Attenuates Neuroinflammation via Regulation the Response to LPS Stimuli In Vitro and In Vivo. Neurochemical Research 37 (7), 1560 1567.

Haass, C., Kaether, C., Thinakaran, G., Sisodia, S., May 2012. Trafficking and Proteolytic Processing of APP. Cold Spring Harbor Perspectives in Medicine 2 (5), a006270-a006270. 
Hahm, K.-B., Cho, K., Lee, C., Im, Y.-H., Chang, J., Choi, S.-G., Sorensen, P. H., Thiele, C. J., Kim, S.-J., 1999. Repression of the gene encoding the TGF- $\beta$ type II receptor is a major target of the EWS-FLI1 oncoprotein. Nature genetics 23 (2), 222-227.

Halder, R., Hennion, M., Vidal, R. O., Shomroni, O., Rahman, R.-U., Rajput, A., Centeno, T. P., van Bebber, F., Capece, V., Vizcaino, J. C. G., Schuetz, A.-L., Burkhardt, S., Benito, E., Sala, M. N., Javan, S. B., Haass, C., Schmid, B., Fischer, A., Bonn, S., Dec. 2015. DNA methylation changes in plasticity genes accompany the formation and maintenance of memory. Nature Neuroscience.

Hall, A. M., Roberson, E. D., May 2012. Mouse models of Alzheimer's disease. Brain Research Bulletin 88 (1), 3-12.

Handoko, L., Xu, H., Li, G., Ngan, C. Y., Chew, E., Schnapp, M., Lee, C. W. H., Ye, C., Ping, J. L. H., Mulawadi, F., Wong, E., Sheng, J., Zhang, Y., Poh, T., Chan, C. S., Kunarso, G., Shahab, A., Bourque, G., CacheuxRataboul, V., Sung, W.-K., Ruan, Y., Wei, C.-L., Jun. 2011. CTCF-mediated functional chromatin interactome in pluripotent cells. Nature Genetics 43 (7), 630-638.

Hardy, J., Jul. 2002. The Amyloid Hypothesis of Alzheimer's Disease: Progress and Problems on the Road to Therapeutics. Science 297 (5580), 353-356.

Harr, J. C., Luperchio, T. R., Wong, X., Cohen, E., Wheelan, S. J., Reddy, K. L., Jan. 2015. Directed targeting of chromatin to the nuclear lamina is mediated by chromatin state and A-type lamins. The Journal of Cell Biology 208 (1), 33-52.

Hayakawa, T., Nakayama, J.-i., 2011. Physiological Roles of Class I HDAC Complex and Histone Demethylase. Journal of Biomedicine and Biotechnology 2011, 1-10.

Hayashi, Y., Kashiwagi, K., Ohta, J., Nakajima, M., Kawashima, T., Yoshikawa, K., Nov. 1994. Alzheimer amyloid protein precursor enhances proliferation of neural stem cells from fetal rat brain. Biochemical and Biophysical Research Communications 205 (1), 936-943.

Hayden, M. S., West, A. P., Ghosh, S., Oct. 2006. NF-кB and the immune response. Oncogene 25 (51), 67586780.

Hedgecock, E. M., Culotti, J. G., Hall, D. H., Jan. 1990. The unc-5, unc-6, and unc-40 genes guide circumferential migrations of pioneer axons and mesodermal cells on the epidermis in C. elegans. Neuron 4 (1), 61-85.

Heintzman, N. D., Stuart, R. K., Hon, G., Fu, Y., Ching, C. W., Hawkins, R. D., Barrera, L. O., Van Calcar, S., Qu, C., Ching, K. A., Wang, W., Weng, Z., Green, R. D., Crawford, G. E., Ren, B., Mar. 2007. Distinct 
and predictive chromatin signatures of transcriptional promoters and enhancers in the human genome. Nature Genetics 39 (3), 311-318.

Hendrich, B., Bird, A., Nov. 1998. Identification and characterization of a family of mammalian methyl-CpG binding proteins. Molecular and Cellular Biology 18 (11), 6538-6547.

Hendriks, L., van Duijn, C. M., Cras, P., Cruts, M., Van Hul, W., van Harskamp, F., Warren, A., McInnis, M. G., Antonarakis, S. E., Martin, J.-J., Hofman, A., Van Broeckhoven, C., Jun. 1992. Presenile dementia and cerebral haemorrhage linked to a mutation at codon 692 of the $\beta$-amyloid precursor protein gene. Nature Genetics 1 (3), 218-221.

Heslegrave, A., Heywood, W., Paterson, R., Magdalinou, N., Svensson, J., Johansson, P., Öhrfelt, A., Blennow, K., Hardy, J., Schott, J., Mills, K., Zetterberg, H., 2016. Increased cerebrospinal fluid soluble TREM2 concentration in Alzheimer’s disease. Molecular Neurodegeneration 11 (1), 3.

Hickman, S. E., El Khoury, J., Apr. 2014. TREM2 and the neuroimmunology of Alzheimer's disease. Biochemical Pharmacology 88 (4), 495-498.

Holroyd, S., Shepherd, M. L., Downs, J. H., 2000. Occipital atrophy is associated with visual hallucinations in Alzheimer's disease. The Journal of Neuropsychiatry and Clinical Neurosciences 12 (1), 25-28.

Holtzer, R., Tang, M.-X., Devanand, D. P., Albert, S. M., Wegesin, D. J., Marder, K., Bell, K., Albert, M., Brandt, J., Stern, Y., 2003. Psychopathological features in Alzheimer's disease: course and relationship with cognitive status. Journal of the American Geriatrics Society 51 (7), 953-960.

Hou, C., Dale, R., Dean, A., Feb. 2010. Cell type specificity of chromatin organization mediated by CTCF and cohesin. Proceedings of the National Academy of Sciences 107 (8), 3651-3656.

Hsu, W.-L., Ma, Y.-L., Hsieh, D.-Y., Liu, Y.-C., Lee, E. H., 2013. STAT1 Negatively Regulates Spatial Memory Formation and Mediates the Memory-Impairing Effect of A $\beta$. Neuropsychopharmacology.

Huang, D. W., Sherman, B. T., Lempicki, R. A., Dec. 2008. Systematic and integrative analysis of large gene lists using DAVID bioinformatics resources. Nature Protocols 4 (1), 44-57.

Huang, D. W., Sherman, B. T., Lempicki, R. A., Jan. 2009. Bioinformatics enrichment tools: paths toward the comprehensive functional analysis of large gene lists. Nucleic Acids Research 37 (1), 1-13.

Huber, W., Carey, J., V., Gentleman, R., Anders, S., Carlson, M., Carvalho, S., B., Bravo, C., H., Davis, S., Gatto, L., Girke, T., Gottardo, R., Hahne, F., Hansen, D., K., Irizarry, A., R., Lawrence, M., Love, I., M., MacDonald, J., Obenchain, V., Ole’s, K., A., Pag‘es, H., Reyes, A., Shannon, P., Smyth, K., G., Tenenbaum, D., Waldron, L., 
Morgan, M., 2015. Orchestrating high-throughput genomic analysis with Bioconductor. Nature Methods 12 (2), 115-121.

Icardi, L., De Bosscher, K., Tavernier, J., Dec. 2012. The HAT/HDAC interplay: Multilevel control of STAT signaling. Cytokine \& Growth Factor Reviews 23 (6), 283-291.

Ihara, Y., Nukina, N., Miura, R., Ogawara, M., Jun. 1986. Phosphorylated tau protein is integrated into paired helical filaments in Alzheimer's disease. Journal of Biochemistry 99 (6), 1807-1810.

Isaacs, G. D., Taher, N., McKenzie, C., Garrett, R., Baker, M., Fox, N., 2013. A $\beta$ Alters the DNA Methylation Status of Cell-fate Genes in an Alzheimer's Disease Model.

Iwata, A., Nagata, K., Hatsuta, H., Takuma, H., Bundo, M., Iwamoto, K., Tamaoka, A., Murayama, S., Saido, T., Tsuji, S., Feb. 2014. Altered CpG methylation in sporadic Alzheimer's disease is associated with APP and MAPT dysregulation. Human Molecular Genetics 23 (3), 648-656.

Iwatsubo, T., Mann, D. M. A., Odaka, A., Suzuki, N., Ihara, Y., Mar. 1995. Amyloid-beta protein (A-beta) deposition: Abeta42(43) precedes Abeta40 in down Syndrome. Annals of Neurology 37 (3), $294-299$.

Jambhekar, S. S., Breen, P., Dec. 2015. Cyclodextrins in pharmaceutical formulations II: solubilization, binding constant, and complexation efficiency. Drug Discovery Today.

Jantaratnotai, N., Utaisincharoen, P., Sanvarinda, P., Thampithak, A., Sanvarinda, Y., Oct. 2013. Phytoestrogens mediated anti-inflammatory effect through suppression of IRF-1 and pSTAT1 expressions in lipopolysaccharide-activated microglia. International Immunopharmacology 17 (2), 483-488.

Jay, T. R., Miller, C. M., Cheng, P. J., Graham, L. C., Bemiller, S., Broihier, M. L., Xu, G., Margevicius, D., Karlo, J. C., Sousa, G. L., Cotleur, A. C., Butovsky, O., Bekris, L., Staugaitis, S. M., Leverenz, J. B., Pimplikar, S. W., Landreth, G. E., Howell, G. R., Ransohoff, R. M., Lamb, B. T., Mar. 2015. TREM2 deficiency eliminates TREM2+ inflammatory macrophages and ameliorates pathology in Alzheimer's disease mouse models. Journal of Experimental Medicine 212 (3), 287-295.

Jenuwein, T., Allis, C. D., Aug. 2001. Translating the histone code. Science (New York, N.Y.) 293 (5532), 10741080.

Jerónimo-Santos, A., Vaz, S. H., Parreira, S., Rapaz-Lérias, S., Caetano, A. P., Buée-Scherrer, V., Castrén, E., Valente, C. A., Blum, D., Sebastião, A. M., Diógenes, M. J., Sep. 2015. Dysregulation of TrkB Receptors and BDNF Function by Amyloid- $\beta$ Peptide is Mediated by Calpain. Cerebral Cortex 25 (9), 3107-3121. 
Jiang, T., Tan, L., Zhu, X.-C., Zhang, Q.-Q., Cao, L., Tan, M.-S., Gu, L.-Z., Wang, H.-F., Ding, Z.-Z., Zhang, Y.-D., Yu, J.-T., Jul. 2014. Up-regulation of TREM2 Ameliorates Neuropathology and Rescues Spatial Cognitive Impairment in a Transgenic Mouse Model of Alzheimer's Disease. Neuropsychopharmacology.

Jin, S. C., Benitez, B. A., Karch, C. M., Cooper, B., Skorupa, T., Carrell, D., Norton, J. B., Hsu, S., Harari, O., Cai, Y., Bertelsen, S., Goate, A. M., Cruchaga, C., Nov. 2014. Coding variants in TREM2 increase risk for Alzheimer's disease. Human Molecular Genetics 23 (21), 5838-5846.

Jolliffe, I. T., 2002. Principal component analysis. Springer, New York.

Jones, B. M., Bhattacharjee, S., Dua, P., Hill, J. M., Zhao, Y., Lukiw, W. J., Mar. 2014. Regulating amyloidogenesis through the natural triggering receptor expressed in myeloid/microglial cells 2 (TREM2). Frontiers in Cellular Neuroscience 8.

Jones, P. A., May 2012. Functions of DNA methylation: islands, start sites, gene bodies and beyond. Nature Reviews Genetics 13 (7), 484-492.

Jonsson, T., Atwal, J. K., Steinberg, S., Snaedal, J., Jonsson, P. V., Bjornsson, S., Stefansson, H., Sulem, P., Gudbjartsson, D., Maloney, J., Hoyte, K., Gustafson, A., Liu, Y., Lu, Y., Bhangale, T., Graham, R. R., Huttenlocher, J., Bjornsdottir, G., Andreassen, O. A., Jönsson, E. G., Palotie, A., Behrens, T. W., Magnusson, O. T., Kong, A., Thorsteinsdottir, U., Watts, R. J., Stefansson, K., Jul. 2012. A mutation in APP protects against Alzheimer's disease and age-related cognitive decline. Nature.

Jonsson, T., Stefansson, H., Steinberg, S., Jonsdottir, I., Jonsson, P. V., Snaedal, J., Bjornsson, S., Huttenlocher, J., Levey, A. I., Lah, J. J., Rujescu, D., Hampel, H., Giegling, I., Andreassen, O. A., Engedal, K., Ulstein, I., Djurovic, S., Ibrahim-Verbaas, C., Hofman, A., Ikram, M. A., van Duijn, C. M., Thorsteinsdottir, U., Kong, A., Stefansson, K., Jan. 2013. Variant of TREM2 Associated with the Risk of Alzheimer's Disease. New England Journal of Medicine 368 (2), 107-116.

Kandel, E. R. (Ed.), 2013. Principles of neural science, 5th Edition. McGraw-Hill, New York.

Kandel, E. R., Tauc, L., Nov. 1965. Heterosynaptic facilitation in neurones of the abdominal ganglion of Aplysia depilans. The Journal of physiology 181 (1), 1-27.

Kang, J., Lemaire, H. G., Unterbeck, A., Salbaum, J. M., Masters, C. L., Grzeschik, K. H., Multhaup, G., Beyreuther, K., Müller-Hill, B., Feb. 1987. The precursor of Alzheimer's disease amyloid A4 protein resembles a cell-surface receptor. Nature 325 (6106), 733-736.

Kar, A., Kuo, D., He, R., Zhou, J., Wu, J. Y., 2005. Tau alternative splicing and frontotemporal dementia. Alzheimer disease and associated disorders 19 (Suppl 1), S29. 
Kawabata, S., Higgins, G. A., Gordon, J. W., Dec. 1991. Amyloid plaques, neurofibrillary tangles and neuronal loss in brains of transgenic mice overexpressing a C-terminal fragment of human amyloid precursor protein. Nature 354 (6353), 476-478.

Kazantsev, A., Thompson, L., 2008. Therapeutic application of histone deacetylase inhibitors for central nervous system disorders. Nature Reviews Drug Discovery 7 (10), 854-868.

Kempf, M., Clement, A., Faissner, A., Lee, G., Brandt, R., 1996. Tau binds to the distal axon early in development of polarity in a microtubule-and microfilament-dependent manner. The Journal of neuroscience 16 (18), 5583-5592.

Khoury, J. E., Hickman, S. E., Thomas, C. A., Cao, L., Silverstein, S. C., Loike, J. D., Aug. 1996. Scavenger receptor-mediated adhesion of microglia to $\beta$-amyloid fibrils. Nature 382 (6593), 716-719.

Kierdorf, K., Erny, D., Goldmann, T., Sander, V., Schulz, C., Perdiguero, E. G., Wieghofer, P., Heinrich, A., Riemke, P., Hölscher, C., Müller, D. N., Luckow, B., Brocker, T., Debowski, K., Fritz, G., Opdenakker, G., Diefenbach, A., Biber, K., Heikenwalder, M., Geissmann, F., Rosenbauer, F., Prinz, M., Jan. 2013. Microglia emerge from erythromyeloid precursors via Pu.1- and Irf8-dependent pathways. Nature Neuroscience 16 (3), 273-280.

Kilgore, M., Miller, C. A., Fass, D. M., Hennig, K. M., Haggarty, S. J., Sweatt, J. D., Rumbaugh, G., 2010. Inhibitors of class 1 histone deacetylases reverse contextual memory deficits in a mouse model of Alzheimer's disease. Neuropsychopharmacology 35 (4), 870-880.

Kohli, R. M., Zhang, Y., Oct. 2013. TET enzymes, TDG and the dynamics of DNA demethylation. Nature 502 (7472), 472-479.

Kos, T., Popik, P., May 2005. A comparison of the predictive therapeutic and undesired side-effects of the NMDA receptor antagonist, memantine, in mice. Behavioural Pharmacology 16 (3), 155-161.

Kosik, K. S., Joachim, C. L., Selkoe, D. J., Jun. 1986. Microtubule-associated protein tau (tau) is a major antigenic component of paired helical filaments in Alzheimer disease. Proceedings of the National Academy of Sciences of the United States of America 83 (11), 4044-4048.

Krabbe, G., Halle, A., Matyash, V., Rinnenthal, J. L., Eom, G. D., Bernhardt, U., Miller, K. R., Prokop, S., Kettenmann, H., Heppner, F. L., Apr. 2013. Functional Impairment of Microglia Coincides with BetaAmyloid Deposition in Mice with Alzheimer-Like Pathology. PLoS ONE 8 (4), e60921.

Ku, C.-J., Hosoya, T., Maillard, I., Engel, J. D., 2012. GATA-3 regulates hematopoietic stem cell maintenance and cell-cycle entry. Blood 119 (10), 2242-2251. 
Kyrylenko, S., Korhonen, P., Kyrylenko, O., Roschier, M., Salminen, A., Aug. 2000. Expression of Transcriptional Repressor Proteins mSin3a and 3b during Aging and Replicative Senescence. Biochemical and Biophysical Research Communications 275 (2), 455-459.

Köpke, E., Tung, Y. C., Shaikh, S., Alonso, A. C., Iqbal, K., Grundke-Iqbal, I., Nov. 1993. Microtubule-associated protein tau. Abnormal phosphorylation of a non-paired helical filament pool in Alzheimer disease. The Journal of Biological Chemistry 268 (32), 24374-24384.

Lachner, M., O’Carroll, D., Rea, S., Mechtler, K., Jenuwein, T., Mar. 2001. Methylation of histone H3 lysine 9 creates a binding site for HP1 proteins. Nature 410 (6824), 116-120.

Lam, B., Masellis, M., Freedman, M., Stuss, D. T., Black, S. E., 2013. Clinical, imaging, and pathological heterogeneity of the Alzheimer's disease syndrome. Alzheimers Res Ther 5 (1), 1.

Lamb, J., Crawford, E. D., Peck, D., Modell, J. W., Blat, I. C., Wrobel, M. J., Lerner, J., Brunet, J.-P., Subramanian, A., Ross, K. N., others, 2006. The Connectivity Map: using gene-expression signatures to connect small molecules, genes, and disease. science 313 (5795), 1929-1935.

Lamonica, J. M., Vakoc, C. R., Blobel, G. A., Dec. 2006. Acetylation of GATA-1 is required for chromatin occupancy. Blood 108 (12), 3736-3738.

Landrieu, I., Lacosse, L., Leroy, A., Wieruszeski, J.-M., Trivelli, X., Sillen, A., Sibille, N., Schwalbe, H., Saxena, K., Langer, T., others, 2006. NMR analysis of a Tau phosphorylation pattern. Journal of the American Chemical Society 128 (11), 3575-3583.

Launer, L. J., Andersen, K., Dewey, M., Letenneur, L., Ott, A., Amaducci, L. A., Brayne, C., Copeland, J. R. M., Dartigues, J.-F., Kragh-Sorensen, P., others, 1999. Rates and risk factors for dementia and Alzheimer's disease results from EURODEM pooled analyses. Neurology 52 (1), 78-78.

Lawrence, M., Huber, W., Pagès, H., Aboyoun, P., Carlson, M., Gentleman, R., Morgan, M., Carey, V., $2013 a$. Software for computing and annotating genomic ranges. PLoS Computational Biology 9.

Lawrence, M., Huber, W., Pagès, H., Aboyoun, P., Carlson, M., Gentleman, R., Morgan, M., Carey, V., $2013 b$. Software for computing and annotating genomic ranges. PLoS Computational Biology 9.

Lee, H.-g., Perry, G., Moreira, P. I., Garrett, M. R., Liu, Q., Zhu, X., Takeda, A., Nunomura, A., Smith, M. A., Apr. 2005. Tau phosphorylation in Alzheimer's disease: pathogen or protector? Trends in Molecular Medicine $11(4), 164-169$.

Leinenga, G., Götz, J., Mar. 2015. Scanning ultrasound removes amyloid- $\beta$ and restores memory in an Alzheimer’s disease mouse model. Science Translational Medicine 7 (278), 278ra33. 
Levy, E., Carman, M. D., Fernandez-Madrid, I. J., Power, M. D., Lieberburg, I., van Duinen, S. G., Bots, G. T., Luyendijk, W., Frangione, B., 1990. Mutation of the Alzheimer's disease amyloid gene in hereditary cerebral hemorrhage, Dutch type. Science 248 (4959), 1124-1126.

Lewandowsky, S., Duncan, M., Brown, G. D. A., Oct. 2004. Time does not cause forgetting in short-term serial recall. Psychonomic bulletin \& review 11 (5), 771-790.

Liao, Y., Smyth, G. K., Shi, W., 2013. The subread aligner: fast, accurate and scalable read mapping by seed-and-vote. Nucleic Acids Research 41, e108.

Lienhard, M., Grimm, C., Morkel, M., Herwig, R., Chavez, L., 2014. Medips: genome-wide differential coverage analysis of sequencing data derived from dna enrichment experiments. Bioinformatics 30, $284-286$.

Liu, M., Wang, S., Yao, W., Zhang, Z., Zhong, X., Sha, L., He, M., Zheng, Z., Wei, M., Jul. 2014. Memantine improves spatial learning and memory impairments by regulating NGF signaling in APP/PS1 transgenic mice. Neuroscience 273, 141-151.

Liu, Z., Hazan-Halevy, I., Harris, D. M., Li, P., Ferrajoli, A., Faderl, S., Keating, M. J., Estrov, Z., Apr. 2011. STAT-3 Activates NF- B in Chronic Lymphocytic Leukemia Cells. Molecular Cancer Research 9 (4), 507515 .

Lockwood, P. L., Apps, M. A. J., Roiser, J. P., Viding, E., Oct. 2015. Encoding of Vicarious Reward Prediction in Anterior Cingulate Cortex and Relationship with Trait Empathy. Journal of Neuroscience 35 (40), 1372013727.

Love, M. I., Huber, W., Anders, S., 2014. Moderated estimation of fold change and dispersion for rna-seq data with deseq2. Genome Biology 15, 550.

Lubin, F. D., Gupta, S., Parrish, R. R., Grissom, N. M., Davis, R. L., Nov. 2011. Epigenetic Mechanisms: Critical Contributors to Long-Term Memory Formation. The Neuroscientist 17 (6), 616-632.

Luger, K., Mäder, A. W., Richmond, R. K., Sargent, D. F., Richmond, T. J., 1997. Crystal structure of the nucleosome core particle at 2.8 Å resolution. Nature 389, 18.

Maechler, M., Rousseeuw, P., Struyf, A., Hubert, M., Hornik, K., 2015. cluster: Cluster Analysis Basics and Extensions. R package version 2.0.3 - For new features, see the 'Changelog' file (in the package source).

Mangialasche, F., Solomon, A., Winblad, B., Mecocci, P., Kivipelto, M., 2010. Alzheimer’s disease: clinical trials and drug development. The Lancet Neurology 9 (7), 702-716. 
Marioni, J. C., Mason, C. E., Mane, S. M., Stephens, M., Gilad, Y., Jul. 2008. RNA-seq: An assessment of technical reproducibility and comparison with gene expression arrays. Genome Research 18 (9), 15091517.

Masters, C. L., Simms, G., Weinman, N. A., Multhaup, G., McDonald, B. L., Beyreuther, K., Jun. 1985. Amyloid plaque core protein in Alzheimer disease and Down syndrome. Proceedings of the National Academy of Sciences of the United States of America 82 (12), 4245-4249.

Mathema, V. B., Koh, Y.-S., Thakuri, B. C., Sillanpää, M., Apr. 2012. Parthenolide, a Sesquiterpene Lactone, Expresses Multiple Anti-cancer and Anti-inflammatory Activities. Inflammation 35 (2), 560-565.

Mattson, M. P., Camandola, S., Feb. 2001. NF-кB in neuronal plasticity and neurodegenerative disorders. Journal of Clinical Investigation 107 (3), 247-254.

Maunakea, A. K., Chepelev, I., Cui, K., Zhao, K., Nov. 2013. Intragenic DNA methylation modulates alternative splicing by recruiting MeCP2 to promote exon recognition. Cell Research 23 (11), 1256-1269.

Maurer, K., Maurer, U., 1998. Alzheimer: das Leben eines Arztes und die Karriere einer Krankheit. Piper, München.

Mazin, P., Xiong, J., Liu, X., Yan, Z., Zhang, X., Li, M., He, L., Somel, M., Yuan, Y., Phoebe Chen, Y.-P., Li, N., Hu, Y., Fu, N., Ning, Z., Zeng, R., Yang, H., Chen, W., Gelfand, M., Khaitovich, P., Apr. 2014. Widespread splicing changes in human brain development and aging. Molecular Systems Biology 9 (1), $633-633$.

McKhann, G., Drachman, D., Folstein, M., Katzman, R., Price, D., Stadlan, E. M., 1984. Clinical diagnosis of Alzheimer's disease Report of the NINCDS-ADRDA Work Group* under the auspices of Department of Health and Human Services Task Force on Alzheimer’s Disease. Neurology 34 (7), 939-939.

Meda, L., Cassatella, M. A., Szendrei, G. I., Otvos, L., Baron, P., Villalba, M., Ferrari, D., Rossi, F., Apr. 1995. Activation of microglial cells by $\beta$-amyloid protein and interferon- $\gamma$. Nature 374 (6523), 647-650.

Metzker, M. L., Jan. 2010. Sequencing technologies - the next generation. Nature Reviews Genetics 11 (1), $31-46$.

Meyer, D. E., Schvaneveldt, R. W., 1971. Facilitation in recognizing pairs of words: Evidence of a dependence between retrieval operations. Journal of Experimental Psychology 90 (2), 227-234.

Miller, G. A., Mar. 1956. The magical number seven plus or minus two: some limits on our capacity for processing information. Psychological review 63 (2), 81-97. 
Mills, J. D., Nalpathamkalam, T., Jacobs, H. I., Janitz, C., Merico, D., Hu, P., Janitz, M., Mar. 2013. RNA-Seq analysis of the parietal cortex in Alzheimer's disease reveals alternatively spliced isoforms related to lipid metabolism. Neuroscience Letters 536, 90-95.

Milward, E. A., Papadopoulos, R., Fuller, S. J., Moir, R. D., Small, D., Beyreuther, K., Masters, C. L., Jul. 1992. The amyloid protein precursor of Alzheimer's disease is a mediator of the effects of nerve growth factor on neurite outgrowth. Neuron 9 (1), 129-137.

Minkeviciene, R., Banerjee, P., Tanila, H., Nov. 2004. Memantine improves spatial learning in a transgenic mouse model of Alzheimer's disease. The Journal of Pharmacology and Experimental Therapeutics 311 (2), 677-682.

Miyamoto, M., Fujita, T., Kimura, Y., Maruyama, M., Harada, H., Sudo, Y., Miyata, T., Taniguchi, T., Sep. 1988. Regulated expression of a gene encoding a nuclear factor, IRF-1, that specifically binds to IFN- $\beta$ gene regulatory elements. Cell 54 (6), 903-913.

Modarresi, F., Faghihi, M. A., Lopez-Toledano, M. A., Fatemi, R. P., Magistri, M., Brothers, S. P., van der Brug, M. P., Wahlestedt, C., Mar. 2012. Inhibition of natural antisense transcripts in vivo results in gene-specific transcriptional upregulation. Nature Biotechnology 30 (5), 453-459.

Moehlmann, T., Winkler, E., Xia, X., Edbauer, D., Murrell, J., Capell, A., Kaether, C., Zheng, H., Ghetti, B., Haass, C., Steiner, H., Jun. 2002. Presenilin-1 mutations of leucine 166 equally affect the generation of the Notch and APP intracellular domains independent of their effect on A 42 production. Proceedings of the National Academy of Sciences 99 (12), 8025-8030.

Mohandas, T., Sparkes, R., Shapiro, L., Jan. 1981. Reactivation of an inactive human X chromosome: evidence for X inactivation by DNA methylation. Science 211 (4480), 393-396.

Montgomery, S. M., Buzsáki, G., 2007. Gamma oscillations dynamically couple hippocampal CA3 and CA1 regions during memory task performance. Proceedings of the National Academy of Sciences 104 (36), 14495-14500.

Morris, R. G., May 1981. Spatial localization does not require the presence of local cues. Learning and Motivation 12 (2), 239-260.

Morsch, R., Simon, W., Coleman, P. D., Feb. 1999. Neurons may live for decades with neurofibrillary tangles. Journal of Neuropathology and Experimental Neurology 58 (2), 188-197. 
Mukrasch, M. D., Bibow, S., Korukottu, J., Jeganathan, S., Biernat, J., Griesinger, C., Mandelkow, E., Zweckstetter, M., Feb. 2009. Structural Polymorphism of 441-Residue Tau at Single Residue Resolution. PLoS Biology 7 (2), e1000034.

Mullan, M., Crawford, F., Axelman, K., Houlden, H., Lilius, L., Winblad, B., Lannfelt, L., Aug. 1992. A pathogenic mutation for probable Alzheimer's disease in the APP gene at the N-terminus of $\beta$-amyloid. Nature Genetics 1 (5), 345-347.

Murray, M. M., Bernstein, S. L., Nyugen, V., Condron, M. M., Teplow, D. B., Bowers, M. T., May 2009. Amyloid $\beta$ Protein: $A \beta 40$ Inhibits A $\beta 42$ Oligomerization. Journal of the American Chemical Society 131 (18), 63166317.

Nardelli, J., Thiesson, D., Fujiwara, Y., Tsai, F.-Y., Orkin, S. H., Jun. 1999. Expression and Genetic Interaction of Transcription Factors GATA-2 and GATA-3 during Development of the Mouse Central Nervous System. Developmental Biology 210 (2), 305-321.

Nathan, C., Calingasan, N., Nezezon, J., Ding, A., Lucia, M. S., La Perle, K., Fuortes, M., Lin, M., Ehrt, S., Kwon, N. S., Chen, J., Vodovotz, Y., Kipiani, K., Beal, M. F., Nov. 2005. Protection from Alzheimer's-like disease in the mouse by genetic ablation of inducible nitric oxide synthase. The Journal of Experimental Medicine 202 (9), 1163-1169.

Nelson, B. D., Bjorkquist, O. A., Olsen, E. K., Herbener, E. S., Dec. 2015. Schizophrenia symptom and functional correlates of anterior cingulate cortex activation to emotion stimuli: An fMRI investigation. Psychiatry Research: Neuroimaging 234 (3), 285-291.

Neuwirth, E., 2014. RColorBrewer: ColorBrewer Palettes. R package version 1.1-2.

Newell-Price, J., Clark, A. J., King, P., May 2000. DNA Methylation and Silencing of Gene Expression. Trends in Endocrinology \& Metabolism 11 (4), 142-148.

Nicolas, C., Peineau, S., Amici, M., Csaba, Z., Fafouri, A., Javalet, C., Collett, V., Hildebrandt, L., Seaton, G., Choi, S.-L., Sim, S.-E., Bradley, C., Lee, K., Zhuo, M., Kaang, B.-K., Gressens, P., Dournaud, P., Fitzjohn, S., Bortolotto, Z., Cho, K., Collingridge, G., Jan. 2012. The JAK/STAT Pathway Is Involved in Synaptic Plasticity. Neuron 73 (2), 374-390.

Noh, H., Seo, H., Apr. 2014. Age-dependent effects of valproic acid in Alzheimer's disease (AD) mice are associated with nerve growth factor (NGF) regulation. Neuroscience 266, 255-265. 
Nosten-Bertrand, M., Errington, M. L., Murphy, K. P. S. J., Tokugawa, Y., Barboni, E., Kozlova, E., Michalovich, D., Morris, R. G. M., Silver, J., Stewart, C. L., Bliss, T. V. P., Morris, R. J., Feb. 1996. Normal spatial learning despite regional inhibition of LTP in mice lacking Thy-1. Nature 379 (6568), 826-829.

OECD, Dec. 2009. Health at a Glance 2009. Health at a Glance. OECD Publishing.

Okano, M., Bell, D. W., Haber, D. A., Li, E., Oct. 1999. DNA Methyltransferases Dnmt3a and Dnmt3b Are Essential for De Novo Methylation and Mammalian Development. Cell 99 (3), 247-257.

Olié, E., Ding, Y., Le Bars, E., de Champfleur, N. M., Mura, T., Bonafé, A., Courtet, P., Jollant, F., Dec. 2015. Processing of decision-making and social threat in patients with history of suicidal attempt: A neuroimaging replication study. Psychiatry Research: Neuroimaging 234 (3), 369-377.

on Aging, N. I., Jun. 2015. Alzheimer’s Disease Medications: Fact sheet. Tech. Rep. 15-3431, National Institutes for Health.

Ott, A., Slooter, A. J. C., Hofman, A., van Harskamp, F., Witteman, J. C. M., Van Broeckhoven, C., Van Duijn, C. M., Breteler, M. M. B., 1998. Smoking and risk of dementia and Alzheimer's disease in a populationbased cohort study: the Rotterdam Study. The Lancet 351 (9119), 1840-1843.

Pages, H., 2016. BSgenome: Infrastructure for Biostrings-based genome data packages and support for efficient SNP representation. R package version 1.38.0.

Parsons, C. G., Stöffler, A., Danysz, W., Nov. 2007. Memantine: a NMDA receptor antagonist that improves memory by restoration of homeostasis in the glutamatergic system - too little activation is bad, too much is even worse. Neuropharmacology 53 (6), 699-723.

Peleg, S., Sananbenesi, F., Zovoilis, A., Burkhardt, S., Bahari-Javan, S., Agis-Balboa, R. C., Cota, P., Wittnam, J. L., Gogol-Doering, A., Opitz, L., Salinas-Riester, G., Dettenhofer, M., Kang, H., Farinelli, L., Chen, W., Fischer, A., 2010. Altered Histone Acetylation Is Associated with Age-Dependent Memory Impairment in Mice. Science 328 (5979), 753-756.

Perez, R. G., Zheng, H., Van der Ploeg, L. H., Koo, E. H., Dec. 1997. The beta-amyloid precursor protein of Alzheimer's disease enhances neuron viability and modulates neuronal polarity. The Journal of Neuroscience: The Official Journal of the Society for Neuroscience 17 (24), 9407-9414.

Pfeifer, G. P., Steigerwald, S., Boehm, T. L., Drahovsky, D., Mar. 1988. DNA methylation levels in acute human leukemia. Cancer Letters 39 (2), 185-192. 
Piccio, L., Buonsanti, C., Cella, M., Tassi, I., Schmidt, R. E., Fenoglio, C., Rinker, J., Naismith, R. T., PaninaBordignon, P., Passini, N., Galimberti, D., Scarpini, E., Colonna, M., Cross, A. H., Jun. 2008. Identification of soluble TREM-2 in the cerebrospinal fluid and its association with multiple sclerosis and CNS inflammation. Brain 131 (11), 3081-3091.

Portela, A., Esteller, M., Oct. 2010. Epigenetic modifications and human disease. Nature Biotechnology 28 (10), 1057-1068.

Portelius, E., Bogdanovic, N., Gustavsson, M. K., Volkmann, I., Brinkmalm, G., Zetterberg, H., Winblad, B., Blennow, K., Aug. 2010. Mass spectrometric characterization of brain amyloid beta isoform signatures in familial and sporadic Alzheimer's disease. Acta Neuropathologica 120 (2), 185-193.

Poulin, S. P., Dautoff, R., Morris, J. C., Barrett, L. F., Dickerson, B. C., Oct. 2011. Amygdala atrophy is prominent in early Alzheimer's disease and relates to symptom severity. Psychiatry Research: Neuroimaging 194 (1), 7-13.

Price, J. L., Morris, J. C., Mar. 1999. Tangles and plaques in nondemented aging and "preclinical” Alzheimer's disease. Annals of Neurology 45 (3), 358-368.

Priller, C., Bauer, T., Mitteregger, G., Krebs, B., Kretzschmar, H. A., Herms, J., Jul. 2006. Synapse Formation and Function Is Modulated by the Amyloid Precursor Protein. Journal of Neuroscience 26 (27), 72127221.

Prince, M., Wimo, A., Guerchet, M., Ali, G.-C., Wu, Y.-T., Prina, M., Aug. 2015. World Alzheimer Report 2015: The Global Impact of Dementia - An Analysis of Prevalence, Incidence, cost and Trends.

Profenno, L. A., Porsteinsson, A. P., Faraone, S. V., Mar. 2010. Meta-Analysis of Alzheimer's Disease Risk with Obesity, Diabetes, and Related Disorders. Biological Psychiatry 67 (6), 505-512.

R Core Team, 2015. R: A Language and Environment for Statistical Computing. R Foundation for Statistical Computing, Vienna, Austria.

Radde, R., Bolmont, T., Kaeser, S. A., Coomaraswamy, J., Lindau, D., Stoltze, L., Calhoun, M. E., Jäggi, F., Wolburg, H., Gengler, S., Haass, C., Ghetti, B., Czech, C., Hölscher, C., Mathews, P. M., Jucker, M., Sep. 2006. Aß42-driven cerebral amyloidosis in transgenic mice reveals early and robust pathology. EMBO reports 7 (9), 940-946.

Rao, P., Benito, E., Fischer, A., 2013. MicroRNAs as biomarkers for CNS disease. Frontiers in Molecular Neuroscience 6. 
Ray, A., Alalem, M., Ray, B. K., Sep. 2013. Loss of Epigenetic Kruppel-like Factor 4 Histone Deacetylase (KLF4-HDAC)-mediated Transcriptional Suppression Is Crucial in Increasing Vascular Endothelial Growth Factor (VEGF) Expression in Breast Cancer. Journal of Biological Chemistry 288 (38), 27232-27242.

Reif, A. E., Allen, J. M., 1964. The AKR thymic antigen and its distribution in leukemias and nervous tissues. The Journal of experimental medicine 120 (3), 413-433.

Ricobaraza, A., Cuadrado-Tejedor, M., Pérez-Mediavilla, A., Frechilla, D., Del Río, J., García-Osta, A., 2009. Phenylbutyrate ameliorates cognitive deficit and reduces tau pathology in an Alzheimer's disease mouse model. Neuropsychopharmacology 34 (7), 1721-1732.

Righolt, C. H., van 't Hoff, M. L. R., Vermolen, B. J., Young, I. T., Raz, V., Dec. 2011. Robust nuclear laminabased cell classification of aging and senescent cells. Aging 3 (12), 1192-1201.

Robakis, N. K., Ramakrishna, N., Wolfe, G., Wisniewski, H. M., 1987. Molecular cloning and characterization of a cDNA encoding the cerebrovascular and the neuritic plaque amyloid peptides. Proceedings of the National Academy of Sciences 84 (12), 4190-4194.

Robertson, K., Wolffe, A., et al., 2000. DNA methylation in health and disease. Nature Reviews Genetics 1 (1), 11-19.

Rockenstein, E. M., McConlogue, L., Tan, H., Power, M., Masliah, E., Mucke, L., 1995. Levels and alternative splicing of amyloid $\beta$ protein precursor (APP) transcripts in brains of APP transgenic mice and humans with Alzheimer’s disease. Journal of Biological Chemistry 270 (47), 28257-28267.

Rodrigues, N. P., Tipping, A. J., Wang, Z., Enver, T., Mar. 2012. GATA-2 mediated regulation of normal hematopoietic stem/progenitor cell function, myelodysplasia and myeloid leukemia. The International Journal of Biochemistry \& Cell Biology 44 (3), 457-460.

Rodríguez-Martín, T., Cuchillo-Ibáñez, I., Noble, W., Nyenya, F., Anderton, B. H., Hanger, D. P., Sep. 2013. Tau phosphorylation affects its axonal transport and degradation. Neurobiology of Aging 34 (9), $2146-2157$.

Rogaev, E. I., Sherrington, R., Wu, C., Levesque, G., Liang, Y., Rogaeva, E. A., Ikeda, M., Holman, K., Lin, C., Lukiw, W. J., others, 1997. Analysis of the 5' Sequence, Genomic Structure, and Alternative Splicing of thepresenilin-1gene (PSEN1) Associated with Early Onset Alzheimer Disease. Genomics 40 (3), $415-424$.

Rohn, T. T., Head, E., Su, J. H., Anderson, A. J., Bahr, B. A., Cotman, C. W., Cribbs, D. H., 2001. Correlation between caspase activation and neurofibrillary tangle formation in Alzheimer's disease. The American journal of pathology 158 (1), 189-198. 
Romero, D., Sanabria-Valentín, E., Vlamakis, H., Kolter, R., Jan. 2013. Biofilm Inhibitors that Target Amyloid Proteins. Chemistry \& Biology 20 (1), 102-110.

Rowland, B. D., Bernards, R., Peeper, D. S., Nov. 2005. The KLF4 tumour suppressor is a transcriptional repressor of p53 that acts as a context-dependent oncogene. Nature Cell Biology 7 (11), 1074-1082.

Rubio, A., Sánchez-Mut, J. V., García, E., Velasquez, Z. D., Oliver, J., Esteller, M., Avila, J., Jan. 2012. Epigenetic control of somatostatin and cortistatin expression by $\beta$ amyloid peptide. Journal of Neuroscience Research 90 (1), 13-20.

Ruthenburg, A. J., Allis, C. D., Wysocka, J., Jan. 2007. Methylation of Lysine 4 on Histone H3: Intricacy of Writing and Reading a Single Epigenetic Mark. Molecular Cell 25 (1), 15-30.

Rösler, M., Retz, W., Retz-Junginger, P., Dennler, H. J., 1998. Effects of two-year treatment with the cholinesterase inhibitor rivastigmine on behavioural symptoms in Alzheimer's disease. Behavioural Neurology 11 (4), 211-216.

Saab, B. J., Luca, R. M., Yuen, W. B., Saab, A. M., Roder, J. C., 2011. Memantine affects cognitive flexibility in the Morris water maze. Journal of Alzheimer's Disease 27 (3), 477.

Sadik, G., Kaji, H., Takeda, K., Yamagata, F., Kameoka, Y., Hashimoto, K., Miyanaga, K., Shinoda, T., 1999. In vitro processing of amyloid precursor protein by cathepsin D. The international journal of biochemistry \& cell biology 31 (11), 1327-1337.

Sakaguchi, M., Kim, K., Yu, L. M. Y., Hashikawa, Y., Sekine, Y., Okumura, Y., Kawano, M., Hayashi, M., Kumar, D., Boyden, E. S., McHugh, T. J., Hayashi, Y., Jun. 2015. Inhibiting the Activity of CA1 Hippocampal Neurons Prevents the Recall of Contextual Fear Memory in Inducible ArchT Transgenic Mice. PLOS ONE 10 (6), e0130163.

Sanchez-Mut, J. V., Aso, E., Panayotis, N., Lott, I., Dierssen, M., Rabano, A., Urdinguio, R. G., Fernandez, A. F., Astudillo, A., Martin-Subero, J. I., Balint, B., Fraga, M. F., Gomez, A., Gurnot, C., Roux, J.-C., Avila, J., Hensch, T. K., Ferrer, I., Esteller, M., Oct. 2013. DNA methylation map of mouse and human brain identifies target genes in Alzheimer's disease. Brain 136 (10), 3018-3027.

Sando, S. B., Melquist, S., Cannon, A., Hutton, M., Sletvold, O., Saltvedt, I., White, L. R., Lydersen, S., Aasly, J., Nov. 2008. Risk-reducing effect of education in Alzheimer's disease. International Journal of Geriatric Psychiatry 23 (11), 1156-1162.

Sarter, M., Bruno, J. P., Feb. 1997. Cognitive functions of cortical acetylcholine: toward a unifying hypothesis. Brain Research Reviews 23 (1-2), 28-46. 
Schenk, D., Barbour, R., Dunn, W., Gordon, G., Grajeda, H., Guido, T., Hu, K., Huang, J., Johnson-Wood, K., Khan, K., Kholodenko, D., Lee, M., Liao, Z., Lieberburg, I., Motter, R., Mutter, L., Soriano, F., Shopp, G., Vasquez, N., Vandevert, C., Walker, S., Wogulis, M., Yednock, T., Games, D., Seubert, P., Jul. 1999. Immunization with amyloid-beta attenuates Alzheimer-disease-like pathology in the PDAPP mouse. Nature 400 (6740), 173-177.

Schmid, C. D., Sautkulis, L. N., Danielson, P. E., Cooper, J., Hasel, K. W., Hilbush, B. S., Sutcliffe, J. G., Carson, M. J., 2002. Heterogeneous expression of the triggering receptor expressed on myeloid cells-2 on adult murine microglia. Journal of neurochemistry 83 (6), 1309-1320.

Scoville, W., Milner, B., 1957. Loss of recent memory after bilateral hippocampal lesions. Journal of Neurology, Neurosurgery \& Psychiatry 20 (1), 11-21.

Senechal, Y., Kelly, P. H., Dev, K. K., Jan. 2008. Amyloid precursor protein knockout mice show agedependent deficits in passive avoidance learning. Behavioural Brain Research 186 (1), 126-132.

Sennvik, K., Boekhoorn, K., Lasrado, R., Terwel, D., Verhaeghe, S., Korr, H., Schmitz, C., Tomiyama, T., Mori, H., Krugers, H., Joels, M., Ramakers, G. J. A., Lucassen, P. J., Van Leuven, F., Mar. 2007. Tau-4r suppresses proliferation and promotes neuronal differentiation in the hippocampus of tau knockin/knockout mice. The FASEB Journal 21 (9), 2149-2161.

Seo, E. H., Choo, I. L. H., Alzheimer's Disease Neuroimaging Initiative, Nov. 2015. Amyloid-independent functional neural correlates of episodic memory in amnestic mild cognitive impairment. European Journal of Nuclear Medicine and Molecular Imaging.

Serafini, T., Colamarino, S. A., Leonardo, E. D., Wang, H., Beddington, R., Skarnes, W. C., Tessier-Lavigne, M., 1996. Netrin-1 is required for commissural axon guidance in the developing vertebrate nervous system. Cell 87 (6), 1001-1014.

Sharrocks, A. D., Nov. 2001. The ETS-domain transcription factor family. Nature Reviews. Molecular Cell Biology 2 (11), 827-837.

Shen, L., Shao, N., Liu, X., Nestler, E., 2014. ngs. plot: Quick mining and visualization of next-generation sequencing data by integrating genomic databases. BMC genomics 15 (1), 284.

Shen, W., Ganetzky, B., Sep. 2009. Autophagy promotes synapse development in Drosophila. The Journal of Cell Biology 187 (1), 71-79. 
Shi, Y., Ikrar, T., Olivas, N. D., Xu, X., Jun. 2014. Bidirectional global spontaneous network activity precedes the canonical unidirectional circuit organization in the developing hippocampus: GNA and hippocampal circuit development. Journal of Comparative Neurology 522 (9), 2191-2208.

Shors, T. J., Townsend, D. A., Zhao, M., Kozorovitskiy, Y., Gould, E., 2002. Neurogenesis may relate to some but not all types of hippocampal-dependent learning. Hippocampus 12 (5), 578-584.

Shumaker, D. K., Dechat, T., Kohlmaier, A., Adam, S. A., Bozovsky, M. R., Erdos, M. R., Eriksson, M., Goldman, A. E., Khuon, S., Collins, F. S., et al., 2006. Mutant nuclear lamin A leads to progressive alterations of epigenetic control in premature aging. Proceedings of the National Academy of Sciences 103 (23), 87038708.

Smith, A. M., Gibbons, H. M., Oldfield, R. L., Bergin, P. M., Mee, E. W., Faull, R. L. M., Dragunow, M., Jun. 2013. The transcription factor PU.1 is critical for viability and function of human brain microglia: Critical Role of PU.1 in Human Microglia. Glia 61 (6), 929-942.

Spencer, W. A., Thompson, R. F., Neilson, D. R., Mar. 1966. Response decrement of the flexion reflex in the acute spinal cat and transient restoration by strong stimuli. Journal of Neurophysiology 29 (2), 221-239.

Stanton, P. K., Winterer, J., Bailey, C. P., Kyrozis, A., Raginov, I., Laube, G., Veh, R. W., Nguyen, C. Q., Müller, W., 2003. Long-term depression of presynaptic release from the readily releasable vesicle pool induced by NMDA receptor-dependent retrograde nitric oxide. The Journal of neuroscience 23 (13), 5936-5944.

Stark, R., Brown, G., 2011. DiffBind: differential binding analysis of ChIP-Seq peak data.

Stecher, J., Müller, W. E., Hoyer, S., 1997. Learning abilities depend on NMDA-receptor density in hippocampus in adult rats. Journal of neural transmission 104 (2-3), 281-289.

Stevens, C. F., Wesseling, J. F., Jan. 1999. Augmentation is a potentiation of the exocytotic process. Neuron 22 (1), 139-146.

Stilling, R. M., Benito, E., Barth, J., Gertig, M., Capece, V., Burckhardt, S., Bonn, S., Fischer, A., Nov. 2014. De-regulation of gene expression and alternative splicing affects distinct cellular pathways in the aging hippocampus. Frontiers in Cellular Neuroscience 8.

Strittmatter, W. J., Weisgraber, K. H., Huang, D. Y., Dong, L.-M., Salvesen, G. S., Pericak-Vance, M., Schmechel, D., Saunders, A. M., Goldgaber, D., Roses, A. D., 1993. Binding of human apolipoprotein E to synthetic amyloid beta peptide: isoform-specific effects and implications for late-onset Alzheimer disease. Proceedings of the National Academy of Sciences 90 (17), 8098-8102. 
Sung, H. Y., Choi, E. N., Ahn Jo, S., Oh, S., Ahn, J.-H., Nov. 2011. Amyloid protein-mediated differential DNA methylation status regulates gene expression in Alzheimer's disease model cell line. Biochemical and Biophysical Research Communications 414 (4), 700-705.

Suzuki, M., Yamada, T., Kihara-Negishi, F., Sakurai, T., Oikawa, T., Nov. 2003. Direct association between PU.1 and MeCP2 that recruits mSin3a-HDAC complex for PU.1-mediated transcriptional repression. Oncogene 22 (54), 8688-8698.

Suzuki, M. M., Bird, A., Jun. 2008. DNA methylation landscapes: provocative insights from epigenomics. Nature Reviews Genetics 9 (6), 465-476.

Takagi, Y., Harada, J., Chiarugi, A., Moskowitz, M. A., Nov. 2002. STAT1 is Activated in Neurons after Ischemia and Contributes to Ischemic Brain Injury. Journal of Cerebral Blood Flow \& Metabolism 22 (11), 13111318.

Tanaka, M., Hibasami, H., Nagai, J., Ikeda, T., Aug. 1980. Effect of 5-azacytidine on DNA methylation in Ehrlich's ascites tumor cells. The Australian Journal of Experimental Biology and Medical Science 58 (4), 391-396.

Tanzi, R. E., Gusella, J. F., Watkins, P. C., Bruns, G. A., St George-Hyslop, P., Van Keuren, M. L., Patterson, D., Pagan, S., Kurnit, D. M., Neve, R. L., Feb. 1987. Amyloid beta protein gene: cDNA, mRNA distribution, and genetic linkage near the Alzheimer locus. Science (New York, N.Y.) 235 (4791), 880-884.

Tekin, S., Mega, M. S., Masterman, D. M., Chow, T., Garakian, J., Vinters, H. V., Cummings, J. L., Mar. 2001. Orbitofrontal and anterior cingulate cortex neurofibrillary tangle burden is associated with agitation in Alzheimer disease. Annals of Neurology 49 (3), 355-361.

Teri, L., Ferretti, L. E., Gibbons, L. E., Logsdon, R. G., McCurry, S. M., Kukull, W. A., McCormick, W. C., Bowen, J. D., Larson, E. B., Jul. 1999. Anxiety in Alzheimer’s Disease: Prevalence and Comorbidity. The Journals of Gerontology Series A: Biological Sciences and Medical Sciences 54 (7), M348-M352.

The-Bioconductor-Dev-Team, 2015. BSgenome.Mmusculus.UCSC.mm10: Full genome sequences for Mus musculus (UCSC version mm10). R package version 1.4.0.

Thomas, S. J., Grossberg, G. T., 2009. Memantine: a review of studies into its safety and efficacy in treating Alzheimer's disease and other dementias. Clinical interventions in aging 4, 367.

Thomas, S. J., Snowden, J. A., Zeidler, M. P., Danson, S. J., Jul. 2015. The role of JAK/STAT signalling in the pathogenesis, prognosis and treatment of solid tumours. British Journal of Cancer 113 (3), 365-371. 
Thompson, R. F., Spencer, W. A., Jan. 1966. Habituation: a model phenomenon for the study of neuronal substrates of behavior. Psychological review 73 (1), 16-43.

Thorndike, E., 1898. Some experiments on animal intelligence. Science, 818-824.

Tilley, L., Morgan, K., Kalsheker, N., Dec. 1998. Genetic risk factors in Alzheimer’s disease. Molecular pathology: MP 51 (6), 293-304.

Tollervey, J. R., Wang, Z., Hortobagyi, T., Witten, J. T., Zarnack, K., Kayikci, M., Clark, T. A., Schweitzer, A. C., Rot, G., Curk, T., Zupan, B., Rogelj, B., Shaw, C. E., Ule, J., Oct. 2011. Analysis of alternative splicing associated with aging and neurodegeneration in the human brain. Genome Research 21 (10), 1572-1582.

Tolwani, R. J., Buckmaster, P. S., Varma, S., Cosgaya, J. M., Wu, Y., Suri, C., Shooter, E. M., 2002. BDNF overexpression increases dendrite complexity in hippocampal dentate gyrus. Neuroscience 114 (3), 795805 .

Tulving, E., Donaldson, W., Bower, G. H., 1972. Episodic and semantic memory. In: Organization of memory. Academic Press, New York, United States, pp. 381-402.

Tulving, E., Patkau, J. E., Jun. 1962. Concurrent effects of contextual constraint and word frequency on immediate recall and learning of verbal material. Canadian journal of psychology 16, 83-95.

Tunnard, C., Whitehead, D., Hurt, C., Wahlund, L. O., Mecocci, P., Tsolaki, M., Vellas, B., Spenger, C., Kloszewska, I., Soininen, H., Lovestone, S., Simmons, A., AddNeuroMed Consortium, Jul. 2011. Apathy and cortical atrophy in Alzheimer's disease. International Journal of Geriatric Psychiatry 26 (7), $741-748$.

Twine, N. A., Janitz, K., Wilkins, M. R., Janitz, M., Jan. 2011. Whole Transcriptome Sequencing Reveals Gene Expression and Splicing Differences in Brain Regions Affected by Alzheimer's Disease. PLoS ONE 6 (1), e16266.

Tyas, S. L., Manfreda, J., Strain, L. A., Montgomery, P. R., Jun. 2001. Risk factors for Alzheimer's disease: a population-based, longitudinal study in Manitoba, Canada. International Journal of Epidemiology 30 (3), 590-597.

Van Cauwenberghe, C., Van Broeckhoven, C., Sleegers, K., Aug. 2015. The genetic landscape of Alzheimer disease: clinical implications and perspectives. Genetics in Medicine.

Varrault, A., Leviel, V., Bockaert, J., 1991. 5-HT1a-sensitive adenylyl cyclase of rodent hippocampal neurons: effects of antidepressant treatments and chronic stimulation with agonists. Journal of Pharmacology and Experimental Therapeutics 257 (1), 433-438. 
Vogel, F., Feb. 1964. A Preliminary Estimate of the Number of Human Genes. Nature 201 (4921), 847-847.

Vorhees, C. V., Williams, M. T., Jul. 2006. Morris water maze: procedures for assessing spatial and related forms of learning and memory. Nature Protocols 1 (2), 848-858.

Waddington, C. H., Feb. 2012. The epigenotype. 1942. International Journal of Epidemiology 41 (1), 10-13.

Waddington, C. H., 2014. The strategy of the genes: a discussion of some aspects of theoretical biology.

Wagner, J. R., Busche, S., Ge, B., Kwan, T., Pastinen, T., Blanchette, M., 2014. The relationship between DNA methylation, genetic and expression inter-individual variation in untransformed human fibroblasts. Genome Biol 15 (2), R37.

Waites, C. L., Craig, A. M., Garner, C. C., 2005. Mechanisms of vertebrate synaptogenesis. Annual review of neuroscience 28, 251-274.

Walker, A., 2015. openxlsx: Read, Write and Edit XLSX Files. R package version 3.0.0.

Wang, C., Gong, B., Bushel, P. R., Thierry-Mieg, J., Thierry-Mieg, D., Xu, J., Fang, H., Hong, H., Shen, J., Su, Z., Meehan, J., Li, X., Yang, L., Li, H., Labaj, P. P., Kreil, D. P., Megherbi, D., Gaj, S., Caiment, F., van Delft, J., Kleinjans, J., Scherer, A., Devanarayan, V., Wang, J., Yang, Y., Qian, H.-R., Lancashire, L. J., Bessarabova, M., Nikolsky, Y., Furlanello, C., Chierici, M., Albanese, D., Jurman, G., Riccadonna, S., Filosi, M., Visintainer, R., Zhang, K. K., Li, J., Hsieh, J.-H., Svoboda, D. L., Fuscoe, J. C., Deng, Y., Shi, L., Paules, R. S., Auerbach, S. S., Tong, W., Aug. 2014. The concordance between RNA-seq and microarray data depends on chemical treatment and transcript abundance. Nature Biotechnology 32 (9), 926-932.

Wang, C., Petryniak, B., Thompson, C., Kaelin, W., Leiden, J., May 1993a. Regulation of the Ets-related transcription factor Elf-1 by binding to the retinoblastoma protein. Science 260 (5112), 1330-1335.

Wang, H., Maurano, M. T., Qu, H., Varley, K. E., Gertz, J., Pauli, F., Lee, K., Canfield, T., Weaver, M., Sandstrom, R., Thurman, R. E., Kaul, R., Myers, R. M., Stamatoyannopoulos, J. A., Sep. 2012. Widespread plasticity in CTCF occupancy linked to DNA methylation. Genome Research 22 (9), 1680-1688.

Wang, Y., Loomis, P. A., Zinkowski, R. P., Binder, L. I., Apr. 1993b. A novel tau transcript in cultured human neuroblastoma cells expressing nuclear tau. The Journal of Cell Biology 121 (2), 257-267.

Warnes, G. R., Bolker, B., Bonebakker, L., Gentleman, R., Liaw, W. H. A., Lumley, T., Maechler, M., Magnusson, A., Moeller, S., Schwartz, M., Venables, B., 2015. gplots: Various R Programming Tools for Plotting Data. R package version 2.17.0. 
Watson, J. D., Crick, F. H. C., Apr. 1953. Molecular Structure of Nucleic Acids: A Structure for Deoxyribose Nucleic Acid. Nature 171 (4356), 737-738.

Watson, L. A., Wang, X., Elbert, A., Kernohan, K. D., Galjart, N., Berube, N. G., Feb. 2014. Dual Effect of CTCF Loss on Neuroprogenitor Differentiation and Survival. Journal of Neuroscience 34 (8), 2860-2870.

Weingarten, M. D., Lockwood, A. H., Hwo, S.-Y., Kirschner, M. W., 1975. A protein factor essential for microtubule assembly. Proceedings of the National Academy of Sciences 72 (5), 1858-1862.

West, R. L., Lee, J. M., Maroun, L. E., 1995. Hypomethylation of the amyloid precursor protein gene in the brain of an Alzheimer's disease patient. Journal of molecular neuroscience: MN 6 (2), 141-146.

Wilcock, G. K., Lilienfeld, S., Gaens, E., Dec. 2000. Efficacy and safety of galantamine in patients with mild to moderate Alzheimer's disease: multicentre randomised controlled trial. Galantamine International-1 Study Group. BMJ (Clinical research ed.) 321 (7274), 1445-1449.

Wilhelm, J., Johnson, G., Haselkorn, R., Geiduschek, E., Mar. 1972. Specific inhibition of bacteriophage SPO1 DNA-directed protein synthesis by the SPO1 transcription factor, TF 1. Biochemical and Biophysical Research Communications 46 (5), 1970-1977.

Winner, B., Kohl, Z., Gage, F. H., Mar. 2011. Neurodegenerative disease and adult neurogenesis: Neurodegenerative disease and adult neurogenesis. European Journal of Neuroscience 33 (6), 1139-1151.

Wirth, M., Madison, C. M., Rabinovici, G. D., Oh, H., Landau, S. M., Jagust, W. J., Mar. 2013. Alzheimer’s Disease Neurodegenerative Biomarkers Are Associated with Decreased Cognitive Function but Not -Amyloid in Cognitively Normal Older Individuals. Journal of Neuroscience 33 (13), 5553-5563.

Wolfe, M. S., Mar. 2009. Tau Mutations in Neurodegenerative Diseases. Journal of Biological Chemistry 284 (10), 6021-6025.

Wolffe, A. P., Jones, P. L., Wade, P. A., 1999. DNA demethylation. Proceedings of the National Academy of Sciences 96 (11), 5894-5896.

Wood, J. G., Mirra, S. S., Pollock, N. J., Binder, L. I., Jun. 1986. Neurofibrillary tangles of Alzheimer disease share antigenic determinants with the axonal microtubule-associated protein tau (tau). Proceedings of the National Academy of Sciences of the United States of America 83 (11), 4040-4043.

Wurster, A. L., Tanaka, T., Grusby, M. J., May 2000. The biology of Stat4 and Stat6. Oncogene 19 (21), 25772584 . 
Xiang, M., Wang, L., Guo, S., Lu, Y.-Y., Lei, H., Jiang, D.-S., Zhang, Y., Liu, Y., Zhou, Y., Zhang, X.-D., Li, H., Jun. 2014. Interferon regulatory factor 8 protects against cerebral ischaemic-reperfusion injury. Journal of Neurochemistry 129 (6), 988-1001.

Xie, R.-L., Gupta, S., Miele, A., Shiffman, D., Stein, J. L., Stein, G. S., van Wijnen, A. J., Jul. 2003. The Tumor Suppressor Interferon Regulatory Factor 1 Interferes with SP1 Activation to Repress the Human CDK2 Promoter. Journal of Biological Chemistry 278 (29), 26589-26596.

Xu, K., Dai, X.-L., Huang, H.-C., Jiang, Z.-F., 2011. Targeting HDACs: A Promising Therapy for Alzheimer's Disease. Oxidative Medicine and Cellular Longevity 2011, 1-5.

Yaffe, K., Vittinghoff, E., Lindquist, K., Barnes, D., Covinsky, K., Neylan, T., Kluse, M., Marmar, C., 2010. Posttraumatic stress disorder and risk of dementia among US veterans. Archives of general psychiatry $67(6), 608$.

Yoshiyama, Y., Higuchi, M., Zhang, B., Huang, S.-M., Iwata, N., Saido, T., Maeda, J., Suhara, T., Trojanowski, J. Q., Lee, V. M.-Y., Feb. 2007. Synapse Loss and Microglial Activation Precede Tangles in a P301s Tauopathy Mouse Model. Neuron 53 (3), 337-351.

Yu, G., Wang, L.-G., He, Q.-Y., 2015. Chipseeker: an r/bioconductor package for chip peak annotation, comparison and visualization. Bioinformatics 31 (14), 2382-2383.

Yu, H., Kortylewski, M., Pardoll, D., Jan. 2007. Crosstalk between cancer and immune cells: role of STAT3 in the tumour microenvironment. Nature Reviews Immunology 7 (1), 41-51.

Yue, J., Wei, W., Lam, C. M. C., Zhao, Y.-J., Dong, M., Zhang, L.-R., Zhang, L.-H., Lee, H.-C., Oct. 2009. CD38/cADPR/Ca2+ Pathway Promotes Cell Proliferation and Delays Nerve Growth Factor-induced Differentiation in PC12 Cells. Journal of Biological Chemistry 284 (43), 29335-29342.

Zambelli, F., Pesole, G., Pavesi, G., Jul. 2009. Pscan: finding over-represented transcription factor binding site motifs in sequences from co-regulated or co-expressed genes. Nucleic Acids Research 37 (Web Server), W247-W252.

Zeisel, A., Munoz-Manchado, A. B., Codeluppi, S., Lonnerberg, P., La Manno, G., Jureus, A., Marques, S., Munguba, H., He, L., Betsholtz, C., Rolny, C., Castelo-Branco, G., Hjerling-Leffler, J., Linnarsson, S., Mar. 2015. Cell types in the mouse cortex and hippocampus revealed by single-cell RNA-seq. Science 347 (6226), 1138-1142. 
Zhang, W., Potrovita, I., Tarabin, V., Herrmann, O., Beer, V., Weih, F., Schneider, A., Schwaninger, M., Jan. 2005. Neuronal activation of NF- $\kappa B$ contributes to cell death in cerebral ischemia. Journal of Cerebral Blood Flow \&\#38; Metabolism 25 (1), 30-40.

Zhang, Y., Iratni, R., Erdjument-Bromage, H., Tempst, P., Reinberg, D., May 1997. Histone Deacetylases and SAP18, a Novel Polypeptide, Are Components of a Human Sin3 Complex. Cell 89 (3), 357-364.

Zhang, Y., Liu, T., Meyer, C. A., Eeckhoute, J., Johnson, D. S., Bernstein, B. E., Nussbaum, C., Myers, R. M., Brown, M., Li, W., Liu, X. S., 2008. Model-based Analysis of ChIP-Seq (MACS). Genome Biology 9 (9), R137.

Zhang, Y., Ng, H. H., Erdjument-Bromage, H., Tempst, P., Bird, A., Reinberg, D., Aug. 1999. Analysis of the NuRD subunits reveals a histone deacetylase core complex and a connection with DNA methylation. Genes \& Development 13 (15), 1924-1935.

Zhao, G.-Y., Li, Z.-Y., Zou, H.-L., Hu, Z.-L., Song, N.-N., Zheng, M.-H., Su, C.-J., Ding, Y.-Q., Aug. 2008. Expression of the transcription factor GATA3 in the postnatal mouse central nervous system. Neuroscience Research $61(4), 420-428$.

Zhao, S., Fung-Leung, W.-P., Bittner, A., Ngo, K., Liu, X., Jan. 2014. Comparison of RNA-Seq and Microarray in Transcriptome Profiling of Activated T Cells. PLoS ONE 9 (1), e78644.

Zhong, Y., Chen, E. Y., Liu, R., Chuang, P. Y., Mallipattu, S. K., Tan, C. M., Clark, N. R., Deng, Y., Klotman, P. E., Ma’ayan, A., He, J. C., May 2013. Renoprotective Effect of Combined Inhibition of Angiotensin-Converting Enzyme and Histone Deacetylase. Journal of the American Society of Nephrology 24 (5), 801-811.

Zhuang, S., Sep. 2013. Regulation of STAT signaling by acetylation. Cellular Signalling 25 (9), 1924-1931. 


\section{Acknowledgments}

'Coming together is a beginning. Keeping together is progress. Working together is success.'

Henry Ford (1863 - 1947)

Three years have passed since I started my PhD. Three years of planning and conducting experiments, analyzing data and interpreting their meaning, digging through the scientific literature and finally writing this thesis. It was a challenging task to finally accomplish my studies, indeed, but even more exciting, cheerful and enlightening. However, it would not have been possible without a lot of nice and helpful people involved I had the pleasure to work and interact with.

First of all: Prof. Dr. André Fischer. Working in his lab was a fruitful experience in many regards and I really appreciated him as a mentor and supervisor. Even though I often had the impression that 24 hours a day are simply not enough for the whole pile of duties he handled, I can hardly remember a single moment where he refused a request and did not have a moment to spare. He was always encouraging, fair and patient, though kindly pushing whenever necessary and I like to express my most sincere gratitude.

I would also like to thank Prof. Dr. Ralf Heinrich and Prof. Dr. Silvio Rizzoli for being a part of my thesis committee and thereby supporting me with helpful advises and ideas. I also appreciate the willingness of Prof. Dr. Dr. Hannelore Ehrenreich, PhD Camin Dean and Dr. Hauke Werner to become members of the examination board and for evaluating my work. Special thanks go to Dr. Stefan Bonn and Vincenzo Capece for providing me with a pipeline for differential gene expression analysis of RNAseq data and for advises on handling and processing NGS-data, and to Dr. Magali Hennion who taught me everything about ChIP and never hesitated to lend me an ear or a helping hand.

My gratitude also goes to the entire Fischer-Lab and former members I was very pleased to spend all that time with and for the really nice working atmosphere. Special thanks go to: Susanne Burkhardt, the good fairy of our group for keeping the lab running and the Illumina sequencer busy with all my samples together with Anna-Lena Schütz from the Bonn-lab; Dr. Rashi Halder for helping me with MeDIP sequencing and data handling; and Gaurav Jain for his aids in processing ChIP data and for all the helpful discussions. 
I would also like to thank Dr. Daniel Riester and Ulrike Kramer who were dealing with all administrative business, making our life at the bench a lot easier. I especially have to thank my fellow PhD student Magdalena Navarro who helped me a lot with conducting the Morris Water Maze experiment, my bench-mate and fellow Hendrik Urbanke, and our former bench-neighbor Ana Martinez Hernandez. Time certainly flied working together with you guys and I really enjoyed our discussions, fun talks and fooling around with you once in a while. I also sincerely appreciated working together with Dr. Eva Benito-Garagorri, who was always eager to help, taught me a lot in the lab, and also reviewed this thesis. You are great.

Apart from all my scientific colleagues who aided me during my work, accompanied or supervised me, I certainly have to acknowledge the support from all my friends and family. It was me who performed the experiments, who squeezed out all the results and who finally compiled it to a thesis. But it was you who were keeping me running. My gratitude to my close friends and PhD-comrades Jan, Michelle, Karl, and Bernard. Getting to know you already during our Bachelor studies (or even the first day of it) and walking the PhD-path together was great. I can not wait to climb the Gänseliesel along with you guys. I also have to thank my old and dearest friends and schoolmates Michael and Stefan. Counting all you guys as my friends, I will never loose confidence because no matter how distant we are, how many mountains there are to climb and seas to cross, you are always right there to support me with everything you've got.

I am also grateful for my dear family, my parents and sisters, who supported me during all my studies in all regards. The entire years spent on studying, however, would have been a complete waste of time without my dearest friend and fellow: my wife, Ulla. Considering all the effort you spent and all the support you gave me, I wouldn't be complete without you. You are the eyes with which I can see clearly, the immune system that keeps me healthy and distressed and the heart that keeps me alive. The three most amazing things happened in my life were meeting you, marrying you and finally founding our own family with our lovely daughter Emily. No matter how nice a day was, it is not a great one without you two and no matter how stressful it was, it doesn’t matter anymore once I see your smile.

Thank you for everything. 


\section{$11 \quad$ Curriculum vitae}

\section{Michael Andre Gertig}

Date of birth June 01,1988 in Aachen

Education

11/2012 - 03/2016 Georg-August-University Göttingen

PhD in the graduate program „Systems Neuroscience“

Amyloid-beta driven changes in transcriptome plasticity: From OMICS to Therapy

10/2010-09/2012 Georg-August-University Göttingen

M.Sc.: „Developmental, Neural, and Behavioral Biology“Study focus: neurobiology

HDAC-inhibition dependent synaptic remodeling and experience-altered learning and memory

in Fmn2-knockout mice - a model for Posttraumatic stress disorder

10/2007-08/2010 Georg-August-University Göttingen

B.Sc.: Biology - Study focus: behavioral-, and neurobiology

The neuronal basis of courtship behavior in Drosophila: the potential role of the neuropeptide SIFamide.

08/1998 - 06/2007 Städtisches Gymnasium Eschweiler

Allgemeine Hochschulreife

Scientific experience

11/2012 - 03/2016 German Center for Neurodegenerative Diseases

PhD student - laboratory for „Epigenetics in neurodegenerative diseases“ in Göttingen

10/2011 -12/2011 University Medical Center Göttingen

Laboratory for aging and cognitive decline

Scientific assistant

03/2012 - 04/2012 Georg-August-University Göttingen

02/2011 - 03/2011 Institute for zoology and anthropolgy

02/2010-03/2010 Student assistant

Publications:

Amyloidosis driven transcriptional changes in the murine hippocampus and anterior cingulate cortex are independent from disruptions in DNA methylation

Gertig MA, Halder R, Burkhardt S, Capece V, Bonn S, Fischer A

In preparation

Early cognitive decline in an APP/PS1 mouse model for Alzheimer's disease cannot be reversed by a combinatory treatment of memantine and the HDAC-inhibitor vorinostat

Gertig MA, Navarro Sala M, Fischer A

In preparation

De-regulation of gene expression and alternative splicing affects distinct cellular pathways in the aging hippocampus

Stilling RM, Benito E, Gertig M, Barth J, Capece V, Burkhardt S, Bonn S, Fischer A

Frontiers in Cellular Neuroscience Vol. 8; 2014-11-13 